\title{
Design of a Hydraulic Dexterous Manipulator for Minimally Invasive Surgery
}

\author{
A DISSERTATION \\ SUBMITTED TO THE FACULTY OF THE GRADUATE SCHOOL \\ OF THE UNIVERSITY OF MINNESOTA \\ BY
}

Devin Rodney Berg

IN PARTIAL FULFILLMENT OF THE REQUIREMENTS FOR THE DEGREE OF

Doctor Of Philosophy

Perry Y. Li and Arthur G. Erdman

September, 2013 
(C) Devin Rodney Berg 2013 SOME RIGHTS RESERVED

This work is licensed under a Creative Commons Attribution 3.0 United States License http://creativecommons.org/licenses/by/3.0/us/ 


\section{Acknowledgments}

I would like to thank the University of Minnesota, the Mechanical Engineering Department, and the Medical Devices Center for their support and resources. I am particularly grateful to my co-advisors Dr. Perry Li and Dr. Art Erdman. Perry has demonstrated a strong commitment to enabling success in his students. He has always found the time to help me through my struggles and encourage me when things were just not working out. Whenever I was faced with a difficult problem, I knew that I could stop in to Perry's office and leave with a new outlook and a good idea for how to approach the problem from a different direction. Art is one of the most supportive individuals I have encountered and has always encouraged me to pursue my professional goals. He has always helped me to see the big picture and keep everything in context. His continued support has made the completion of this dissertation possible and has helped me to keep moving forward both professionally and personally.

I would also like to thank all of my friends and colleagues who have given me advice or helped me in numerous ways over the years (in no particular order): Dr. Tim Kinney, Dr. Frank Kelso, Pat Nelson, Lucas Harder, Dr. Sue Mantell, Joe Janiszewski, Mitsuhiro Oura, Felicity Pino, Peter Zimmermann, Dr. Tianhong Cui, Dr. David Nuckley, Dr. James Van de Ven, Greg Slupe, Dr. John Petro, and Dr. Caleb Rucker. Additionally, I would like to acknowledge the Institute for Engineering in Medicine at the University of Minnesota for funding this work, 3M 
and the University of Minnesota for awarding me the 3M Science and Technology Fellowship, and the University of Minnesota Medical Devices Center for providing access to valuable tools and materials.

Similarly, the help and input of my dissertation committee has been greatly appreciated. Those individuals include: Dr. Perry Li, Dr. Art Erdman, Dr. Tim Kowalewski, Dr. Barry Kudrowitz, and Dr. Will Durfee. By making themselves available for conversations about particular aspects of this research, they have helped me in ways I can not adequately express.

Lastly, I would like to thank my family, and in particular my wife Lauren, for their love, encouragement, and understanding. Lauren's support during these past few years have made this dissertation possible and without her, I am sure that I would not have finished. 


\section{Dedication}

To my wife and daughter, my inspiration and motivation. 


\title{
Design of a Hydraulic Dexterous Manipulator for Minimally Invasive Surgery
}

\author{
by Devin Rodney Berg \\ ABSTRACT
}

The research described here identifies the limitations of existing robotic surgical platforms, which include the balance between the scale of the robot and its manipulability in terms of range of motion, load capacity, and tool capability, then develops a means of overcoming them by taking advantage of fluid power as an enabling technology with its inherent power density and controllability. The approach described here differs significantly from conventional surgical robots in that the robot is embedded within the surgical device itself, whereas in the conventional system, a general-purpose robot is used to manipulate various surgical tools. This is done in order to demonstrate that fluid power can be used advantageously for the design of embedded surgical robotic systems for minimally invasive surgery.

To enable the design of a fluid powered surgical robot, it was first necessary to identify the design requirements for a robot of this nature as well as the considerations unique to this approach. To this end, a quantification of the necessary load capacity for natural orifice robots was conducted. Further, through a review of the literature in the fields of surgery and robotics, considerations of necessary workspace and limitations for the prevention of tissue damage were explored. The results of these analyses are presented. 
The technologies that comprise this novel surgical robotic system include a hydraulic control valve, actuation units, and an enabling structure. The intended application of these technologies introduced numerous limitations and challenges to the design process. The most stringent of these limitations was that of overall size, due to the realities of patient anatomy, which prevented the use of commercially available hydraulic components. An assemblage of components to achieve the aforementioned design requirements is described including the design of a novel hydraulic control valve to enable manipulation of three actuators using a single valve sized to fit within the working channel of a surgical endoscope.

The advantage of the described approach is that the device enables greater miniaturization, improves cost effectiveness, and has better ease of mobility. The mobility and the relaxed requirements for operating room cleanliness can be potentially useful for mobile clinics, out-patient clinical settings, and on the battlefield. Being more cost effective and having a small overall size, the robotic assisted surgical devices can be widely deployed, even in rural or other less technology intensive environments. Through careful review of the literature and analytical evaluation of the various proposed concepts, it was possible to arrive at a design that meets the needs of modern surgical interventions while addressing the perceived limitations of existing surgical robotics.

Through the efforts described in this dissertation, much new information was produced and developments resulted. The considerations of hydraulic power for surgical robots were evaluated and are applicable to other surgical tasks where hydraulic power may be used advantageously. A quantification of the load requirements for surgical robots performing abdominal procedures was produced which will provide a guide for other researchers developing surgical robots. These values are difficult to find in the literature and are a valuable resource for the field. An alternative, simplified model for predicting the behavior of continuum beams under load was developed to provide an inverse formulation for computing beam shape and end loads. This is useful as continuum beams are widely used for minimally 
invasive surgical manipulators as well as in a wide variety of other applications. Finally, a novel valve concept and two possible designs realizing this concept were developed. These valve designs facilitate control over the three actuators in an antagonistic arrangement. Further, the valve designs enable proportional control of the three actuators at a size scale not commercially available. In summary, the design of a novel hydraulic surgical manipulator as a summation of its parts has been performed. This design demonstrates the feasibility of the fluid power approach to embedded minimally invasive surgical robotics. The pursuit of this research has provided many unique challenges and the work presented here has addressed many of them, as well as laid the foundation for future developments in the application of hydraulic power to the growing field of surgical robotics for minimally invasive surgery. 


\section{Contents}

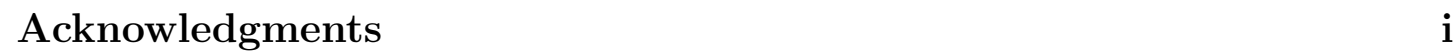

Dedication iii

Abstract iv

List of Tables $\quad$ xi

List of Figures $\quad$ xii

\begin{tabular}{lll}
\hline & Introduction & 1
\end{tabular}

1.1 Background and Related work . . . . . . . . . . . . . . . . . . 1

$1.1 .1 \quad$ Open Surgery . . . . . . . . . . . . . . . . . 2

1.1 .2 Laparoscopic Surgery . . . . . . . . . . . . . . . . . . 2

1.1.3 Single Port Access Surgery . . . . . . . . . . . . . . . 4

1.1 .4 Natural Orifice Surgery . . . . . . . . . . . . . . . . . . . 5

1.1 .5 Robotics in Surgery . . . . . . . . . . . . . . . . 7

1.2 Motivation . . . . . . . . . . . . . . . . . . . . 17

1.3 Problem Description

1.4 Vision . . . . . . . . . . . . . . . . . . . . . . . . . . 21

1.5 Dissertation Overview and Contributions . . . . . . . . . . . . . 26 
2 Design Requirements 30

2.1 Tool Force Requirements . . . . . . . . . . . . . . . . . . . . . . . 31

2.1 .1 Motivation . . . . . . . . . . . . . . . . 31

2.1 .2 Methods . . . . . . . . . . . . . . . . . 32

2.1 .3 Results . . . . . . . . . . . . . . . . . . 36

2.1 .4 Discussion $\ldots \ldots \ldots$. . . . . . . . . . . . . . . . . . . 37

2.1 .5 Conclusions . . . . . . . . . . . . . . . . . . . . . . . 39

2.2 Workspace . . . . . . . . . . . . . . . . . . . 40

2.3 Safety Considerations . . . . . . . . . . . . . . . . . . . . . . 41

2.4 Tool Size Constraints . . . . . . . . . . . . . . . . . . . . . . 44

2.5 Summary of Design Requirements . . . . . . . . . . . . . . . . 45

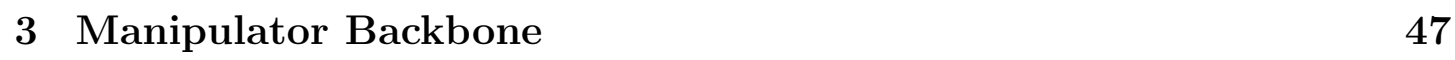

3.1 Motivation and Related Work . . . . . . . . . . . . . . . . . . . . 47

3.2 Simplified Euler-Bernoulli Beam . . . . . . . . . . . . . . . . . . . 49

3.3 Model Prediction of Beam Shape and End Loads . . . . . . . . . 53

3.3 .1 IFM in Two-Dimensions . . . . . . . . . . . . . . . 54

3.3 .2 IFM in Three-Dimensions . . . . . . . . . . . . . . . . . 60

3.4 Experimental Model Validation . . . . . . . . . . . . . . . . . 66

3.5 Summary of Manipulator Backbone Model . . . . . . . . . . . . . 79

4 Artificial Muscle Actuation Unit 82

4.1 Motivation and Related Work . . . . . . . . . . . . . . . . . . . . 82

4.2 Analytical Description of Artificial Muscle Actuators . . . . . . . 84

4.3 Braid Parameter Selection . . . . . . . . . . . . . . . . . . . . . 89

$4.3 .1 \quad$ Finite Element Analysis of Internal Bladder . . . . . . . . 90 
4.3.2 $\quad$ Parameter Selection Methodology and Fabrication . . . . . 99

4.4 Testing of AMA Load Capacity . . . . . . . . . . . . . . . . . . . 100

4.5 Summary of Actuation Unit Design . . . . . . . . . . . . . . . 102

$\begin{array}{lll}5 \text { Control Valve } & 105\end{array}$

5.1 Motivation and Related Work . . . . . . . . . . . . . . . . . 106

5.2 Control Valve Design . . . . . . . . . . . . . . . . . . . . . . . . . 108

$5.3 \quad$ Analytical Description of Flapper Based Valve Design . . . . . . . 111

5.4 Valve Actuation . . . . . . . . . . . . . . . . . . . . . . . . 113

$5.4 .1 \quad$ Piezoelectric Actuation . . . . . . . . . . . . . . . . . . . . 114

$5.4 .2 \quad$ Electromagnetic Actuation . . . . . . . . . . . . . . . 115

5.5 Description of Valve Realizations . . . . . . . . . . . . . . . . . . 120

$5.5 .1 \quad$ Plug-Based Valve Design . . . . . . . . . . . . . . . . . . . 121

5.5 .2 Ring-Based Valve Design . . . . . . . . . . . . . . . . . . 122

5.5 .3 Valve Connection for Supply and Return Lines. . . . . . . 124

$5.6 \quad$ Valve Prototyping and Experimental Results . . . . . . . . . . . . 128

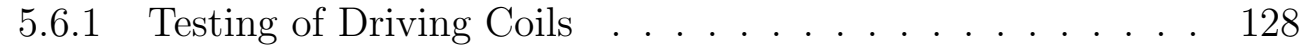

5.6 .2 Valve Prototype Testing . . . . . . . . . . . . . . . . . . 129

5.7 Summary of Control Valve Design . . . . . . . . . . . . . . . . 135

$\begin{array}{lll}6 & \text { System Assembly } & 137\end{array}$

6.1 Motivation for System Level Analysis . . . . . . . . . . . . . . . . 138

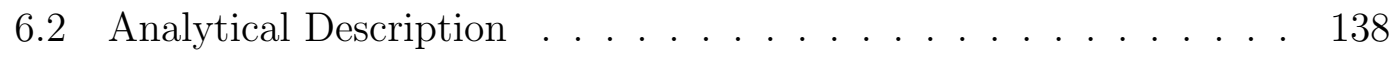

6.2 .1 System Model in Two Dimensions . . . . . . . . . . . . . . 139

$6.2 .2 \quad$ System Model in Three Dimensions . . . . . . . . . . . . . 145

6.2 .3 General Solution Method . . . . . . . . . . . . . . . . . 150 
6.3 Component Specifications … . . . . . . . . . . . . . 151

$6.3 .1 \quad$ Manipulator Backbone . . . . . . . . . . . . . . . . 152

6.3 .2 Actuation Unit . . . . . . . . . . . . . . . . . . . 154

6.3 .3 Control Valve . . . . . . . . . . . . . . . . . . 157

6.4 System Analysis . . . . . . . . . . . . . . . . . . . . . . . . . . . 158

6.4 .1 Theoretical System Analysis . . . . . . . . . . . . . . 158

6.4 .2 Computer Rendered System Assembly _. . . . . . . . . 167

$6.4 .3 \quad$ System Prototyping and Testing . . . . . . . . . . . . . 170

6.5 Summary of System Assembly . . . . . . . . . . . . . . 176

7 Conclusions and Future Work 179

7.1 Review . . . . . . . . . . . . . . . . . . . . . . . . . . . . . . . 179

7.2 Conclusions . . . . . . . . . . . . . . . . . . . . . . . 182

7.3 Future Work . . . . . . . . . . . . . . . . . . . . . . . . 187

$7.3 .1 \quad$ Prototype Iteration $\ldots \ldots \ldots \ldots \ldots$

$7.3 .2 \quad$ Sensing and Control _. . . . . . . . . . . . . . . . . . 188

$7.3 .3 \quad$ User Interface $\ldots \ldots \ldots$

\begin{tabular}{ll}
\hline References & 192
\end{tabular}

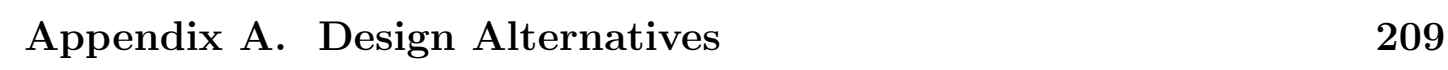

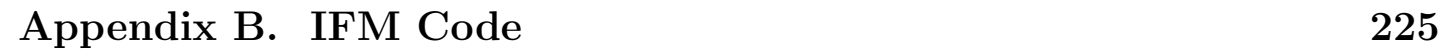

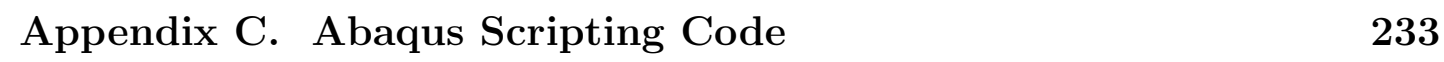




\section{List of Tables}

$2.1 \quad$ Suture sizes and types evaluated for break force . . . . . . . . . . 34

2.2 Abdominal probing force measurements . . . . . . . . . . . . . . . 36

2.3 Suture task force measurements . . . . . . . . . . . . . . . . . . . 37

2.4 Suture break force measurements. . . . . . . . . . . . . . . . . . . 37

3.1 Distal end location and orientation conditions arranged according to corresponding sub-figure references. . . . . . . . . . . 67

3.2 Model predicted end loads using both the full potential energy function (Eq. 3.6) and the simplified energy function (Eq. 3.20). Prediction of stored potential energy, $P E^{*}$, also included for comparison. Forces are given in $N$, moments and potential energy in $N \cdot m$. 68

3.3 Experimental versus model predicted end loads for a sixth-order polynomial model and a 28-segment spline model. Forces are given in $\mathrm{N}$ and moments in $N \cdot m . . \ldots \ldots$

3.4 Coefficient of determination, total residual error, and maximum residual error calculated for each sub-figure. Values presented for both the sixth order polynomial and 28-segment spline parameterizations. Parameterization with higher $R^{2}$ value for each dataset is indicated by boldface type. . . . . . . . . . . . 78

5.1 Comparison of PZ stack with and without amplification. . . . . . 115

$6.1 \quad$ Results from manipulator stiffness measurements. . . . . . . . . . 175 


\section{List of Figures}

1.1 Example of laparoscopic surgery showing access ports and a visu-

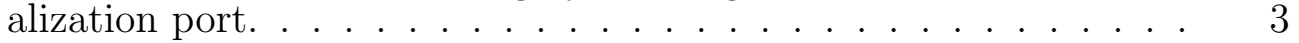

$1.2 \quad$ Example of single port access surgery. . . . . . . . . . . . . . . . . 5

1.3 Example of transgastric natural orifice surgery. . . . . . . . . . . . 6

1.4 da Vinci Surgical System including surgeon console (left) and patient cart (right). ................... 8

1.5 Endoscopic robot design described by Slatkin et al. . . . . . . . . 10

1.6 Envisioned design of the robotic surgical tool for natural orifice

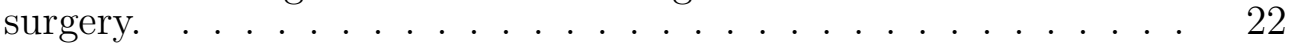

1.7 Model of the robotic surgical tool design shown with two manipu-

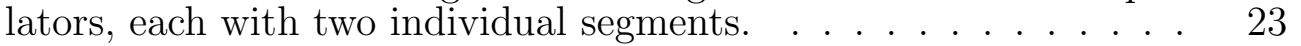

1.8 Line drawings showing a minimal version of how the valve, actuator, and tendon components are connected for (a) a single-segment manipulator and (b) a multi-segment manipulator. $\ldots . . . . .24$

1.9 Illustration of the robotic design during a procedure showing access to the abdominal cavity gained through an incision in the stomach

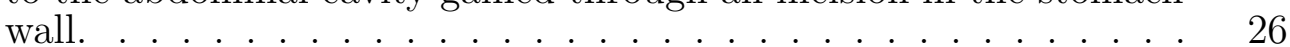

2.1 Illustration of the mounted six-axis transducer . . . . . . . . . . . 33

2.2 Force measurements being taken in a porcine abdomen . . . . . . 34

2.3 Evaluation of suture knot tightening force requirements . . . . . . 35

2.4 Rigidly mounted suture for measuring break force . . . . . . . . . 36

2.5 Example of tissue damage due to applied pressure. . . . . . . . . . . 42 
2.6 Example layout of endoscope cross-section with necessary channels. The manipulators described here would be contained within the tool channels. . . . . . . . . . . . . . . . . . . . . 45

3.1 Graphic of beam bending based design. . . . . . . . . . . . . . . . 49

3.2 Resulting beam geometry from an end applied moment. . . . . . . 51

3.3 Graphic showing location of the beam tip. . . . . . . . . . . . . 52

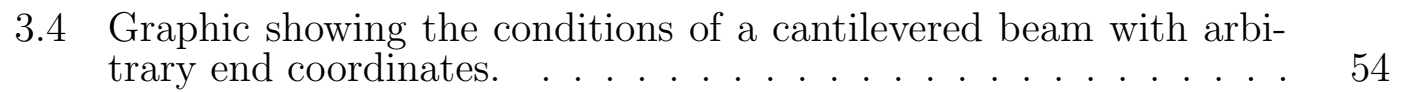

3.5 Example backbone model output for arbitrary input parameters. . $\quad 58$

3.6 Comparison of IFM output with that of constant curvature model for constant curvature input conditions. . . . . . . . . . . . . 59

3.7 Three dimensional Cartesian coordinate system showing orientation angles for a differential beam segment, $d s$. The $y^{\prime}$-axis represents the new, rotated $\mathrm{y}$-axis and the $z^{\prime \prime}$-axis represents the new rotated z-axis. . . . . . . . . . . . . . . . . . . . 61

3.8 Example of three-dimensional IFM output for arbitrary input pa-

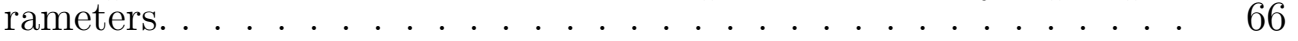

3.9 Example photograph of experimental setup used to record beam shape for arbitrary position and orientation of distal end. . . . . . 67

3.10 A set of six plots showing the output of the IFM using the simplified energy function compared against the IFM using the full energy

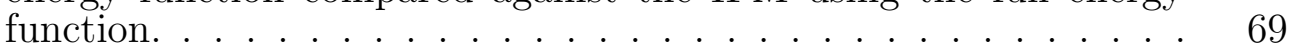

3.11 A set of six plots showing the output of the IFM with 6th order polynomial parameterization compared against the experimentally determined beam shape..$\ldots \ldots \ldots$. . . . . . . . . 71

3.12 A set of six plots showing the output of the IFM using a 6th order polynomial, an 8th order polynomial, and the experimentally determined beam shape. . . . . . . . . . . . . . .

3.13 A set of six plots showing the output of the IFM using spline parameterizations. 6-segment, 8-segment, 10-segment, and 28-segment. 73

3.14 A set of six plots comparing the output of the IFM using 6th order polynomial parameterization versus the 28-segment spline param-

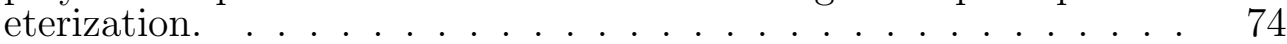


3.15 Error between experimental and model predicted end loads for both 6 th order polynomial and 28-segment spline parameterizations plotted against strain energy calculated trom the 6th order polynomial parameterization for each of the tested experimental conditions. . 76

3.16 Graphic illustrating the variables used in the calculation of the coefficient of determination. . . . . . . . . . . . . . . . 78

4.1 Artificial muscle actuator. . . . . . . . . . . . . . . . . . . . . 84

4.2 Artificial muscle actuator geometry (left) and magnified view of braid geometry relationship for a single unwound strand (right). . 85

4.3 Ratio of force capacity for artificial muscle actuator to force capacity of a piston and cylinder when the maximum cross-sectional area of each actuator is equivalent $\left(F=P \cdot A_{\max }\right)$ as a function of braid angle. For the artificial muscle actuator, the maximum diameter is

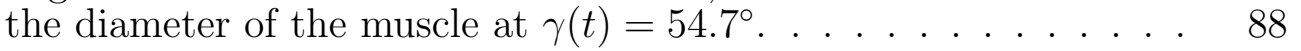

4.4 Illustration of the bladder mesh bulging through a gap in the strands of the braided sheath. . . . . . . . . . . . . . . 92

4.5 Graphic showing relevant planar dimensions describing an individual bladder segment. $\ldots \ldots \ldots . \ldots . \ldots . \ldots 92$

4.6 Example output of Abaqus simulation results for deformation of an internal bladder section. . . . . . . . . . . . . . . . . . . . . 94

4.7 $\quad$ Example output of Abaqus simulation results for Von Mises stress of an internal bladder section. . . . . . . . . . . . . . . 95

4.8 Calculation of maximum bladder stress for variable braid angle. Values indicated in the legend correspond to $\left[P_{a}(\mathrm{MPa}), N, t(\mathrm{~mm})\right] .97$

4.9 Plot of the bladder stress calculated using FEA versus (a) actuator pressure, (b) strand number, and (c) bladder wall thickness. Braid angle is set to $45^{\circ}$ for all cases. . . . . . . . . . . . . . 98

4.10 Photograph of a prototype artificial muscle actuator. . . . . . . . 101

4.11 Illustration of test setup for measuring actuator load as a function

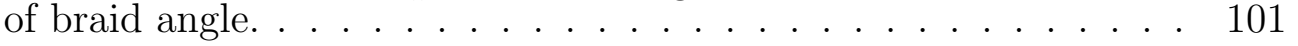

4.12 Plot of force output from the artificial muscle actuator predicted analytically (solid line) and determined experimentally (dots). . . 102

$5.1 \quad$ Hydraulic circuit concept for control of a single actuator. . . . . . 109 
5.2 Three actuator hydraulic circuit concept for the flapper style valve

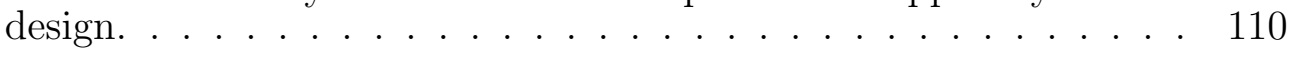

5.3 Plot of pressure ratio versus orifice ratio for a flapper valve. . . . . 113

5.4 Photograph of a 44 gauge wire coil used to test heat dissipation. . 117

5.5 Exploded view of the plug-based valve model showing the valve

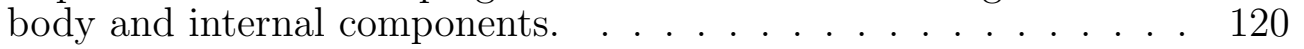

5.6 Model of ring based flapper valve design. . . . . . . . . . . . . . . 121

5.7 Enlarged view of plug-based valve showing downstream orifices and

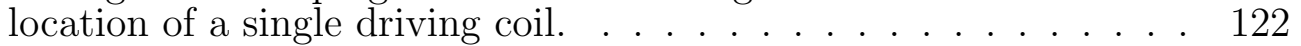

5.8 Model of the control valve showing part of the flow path for both open (top) and blocked (bottom) variable orifice conditions. . . . 123

5.9 Model of the ring-based control valve showing part of the flow path for both blocked (top) and open (bottom) variable orifice conditions. 124

5.10 Illustration of the ring-based valve showing the taper angle. . . . . 125

5.11 Example cross-section of a multi-lumen tubing used to provide both feed and return lines for the control valve. . . . . . . . . . . . . . 126

5.12 Illustration of valve connection to the hydraulic supply and return lumens of the described tubing for the (a) plug-based valve design and (b) the ring-based valve design. . . . . . . . . . . . . . 127

5.13 Photograph of the design scale prototype of the plug-based valve

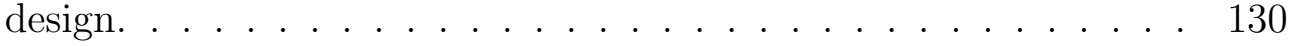

5.14 Photographs showing the scaled plug-based valve prototype in both (a) manually manipulated and (b) electromagnetically manipulated configurations. . . . . . . . . . . . . . . . . 131

5.15 Photograph of hydraulic supply setup. . . . . . . . . . . . . . . . 132

5.16 Machined prototype of the second valve design. . . . . . . . . . . 133

5.17 Photographs showing the scaled valve prototype for the ring-based design with (a) outlet and supply lines attached and (b) assembled within the return line. . . . . . . . . . . . . . . 134

6.1 Graphic showing angle change of tendon due to routing through intermediate disks. . . . . . . . . . . . . . . . . . . . . . . . . . 139 
6.2 Graphic showing resulting free body diagram for assumption of many intermediate disks. . . . . . . . . . . . . . . 140

6.3 Skeleton model showing the basic components that make up the two-dimensional system. $\ldots \ldots \ldots$. . . . . . . . . . . . . 141

6.4 Simplified block diagram of system model. . . . . . . . . . . . . . 142

6.5 A differential segment of the manipulator with the backbone and tendon shown. . . . . . . . . . . . . . . . . 143

6.6 Free body diagram for mass at the distal end of the manipulator. 144

6.7 Illustration of the terminal disk showing the tendon connection points as indicated by the black squares. . . . . . . . . . . 146

6.8 Simplified block diagram of the 3D system model. . . . . . . . . . 147

6.9 A differential three-dimensional segment of the manipulator with the backbone and tendon shown. . . . . . . . . . . . . . . . . . . 148

6.10 Free body diagram for the three-dimensional mass at the distal end of the manipulator. . . . . . . . . . . . . . . . . . . . 150

6.11 A set of five plots showing the planar working envelope of a two segment manipulator for a per segment bend range of (a) $30^{\circ}$, (b) $60^{\circ}$, (c) $90^{\circ}$, (d) $120^{\circ}$, and (e) $180^{\circ} . \ldots \ldots \ldots$

6.12 Photograph of a muscle actuator braiding process setup. . . . . . 156

6.13 Photograph of braid sample at 15 pick per inch. . . . . . . . . . . 156

6.14 Plot of tool tip orientation versus actuator pressure for planar bending using the two dimensional model. . . . . . . . . . . . . . . . . 160

6.15 Plot of planar beam deflection for increasing actuator pressure with no external load for the two dimensional model. . . . . . . . . . . 161

6.16 Plot of tool tip orientation versus actuator pressure for planar bending using the three dimensional model. . . . . . . . . . . . . . 162

6.17 (a) Illustration of valve flapper in-plane position change where $\begin{array}{ll}\text { dashed lines correspond to orientation of each downstream orifice. } & 163\end{array}$

6.18 Plot of (a) gap size for both the active and antagonistic actuators and (b) tool tip orientation versus time. $\ldots \ldots \ldots$. . . . . 163 
6.19 Plot of (a) actuator pressure and (b) actuator length versus tool tip orientation for simulation with increased actuator stiffness. . . 164

6.20 Plot of beam deflection for varying actuator pressures applied to each of the three actuators with no external load. . . . . . . . . . 164

6.21 Simulation of three dimensional beam deflection through activation of two actuators. $\ldots \ldots \ldots \ldots$. . . . . . . . . . . . 165

6.22 Actuator pressures for each of the activated actuators. . . . . . . 166

6.23 Calculated beam shape for a beam deflected by a single actuator with and without an end load applied. . . . . . . . . . . . . 167

6.24 Calculated beam shape for a beam deflected by an end load with

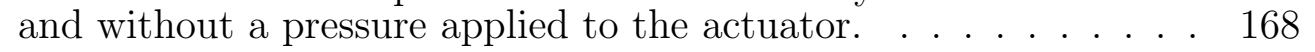

6.25 Computer rendering showing the full manipulator assembly in (a) perspective view and (b) side view, (c) the internal components used to operate a single segment of one manipulator, and (d) the connection between the valve and a single actuator shown in its inflated state. . . . . . . . . . . . . . 169

6.26 Illustration of the cross section of the working channel of an endoscope showing three actuators arranged in parallel, one of which is fully inflated and the routing channels for the manipulator tendons. 170

6.27 Illustration of the valve within the working channel of an endoscope with channels providing connection to the suction and irrigation lines. 171

6.28 Computer model of design scale, two segment per manipulator sys-

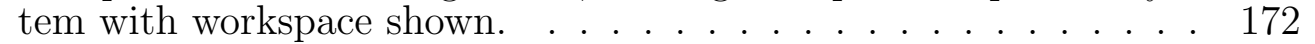

6.29 Photograph of a pressure regulated syringe and manipulator testing

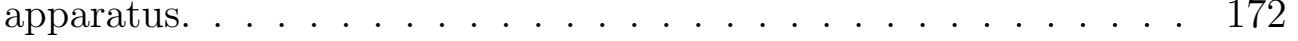

6.30 Photograph of manipulator backbone under load. . . . . . . . . . 174

6.31 Illustration of test setup for measurement of manipulator stiffness. 175

6.32 Computer rendering showing internal system components for a single manipulator section in (a) an exploded view, (b) assembled in place, and (c) assembled within the working channel of an endoscope. 178

A.1 Jointed support structure. . . . . . . . . . . . . . . . . . . . . 210

A.2 Sketch showing the labeled geometric references used in later equa-

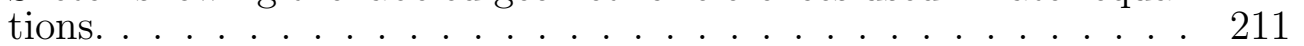


A.3 $\quad$ Free body diagram of a sample three-link joint. . . . . . . . . . . 212

A.4 Demonstration of problematic joint response. . . . . . . . . . . . . 214

A.5 Example of a pressure balanced valve concept. . . . . . . . . . . . 215

A.6 Geometry of a single set of comb-drive fingers. . . . . . . . . . . . 216

A.7 Folded flexure design commonly used for large displacement elec-

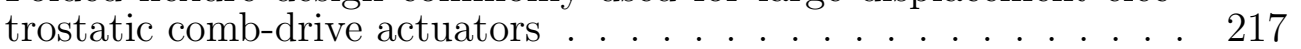

A.8 Mask layout for valve design. . . . . . . . . . . . . . . . . . . . . 219

A.9 Key to be used to identify materials in the process design figures. 220

A.10 Bare silicon wafer. . . . . . . . . . . . . . . . . . . . 220

A.11 Wafer after silicon nitride deposition. . . . . . . . . . . . . . . . . 220

A.12 Wafer after silicon dioxide deposition. . . . . . . . . . . . . . . . . 221

A.13 Wafer after silicon dioxide etching. . . . . . . . . . . . . . . . . . 221

A.14 Wafer after polysilicon deposition. . . . . . . . . . . . . . . . . . . 221

A.15 Wafer after polysilicon etching. . . . . . . . . . . . . . . . . . . . 222

A.16 Wafer after gold deposition. . . . . . . . . . . . . . . . . . . . 222

A.17 Wafer after removal of sacrificial layer. . . . . . . . . . . . . . 223

A.18 Valve design prototype shown at 5x magnification. . . . . . . . . . 223 


\section{Chapter 1}

\section{Introduction}

As the field of surgery developed through the centuries from early experimentation to the advanced procedures common today, so too have the design and implementation of novel surgical tools. This introductory chapter first briefly lays out the changes observed and the changes that are yet in progress in the surgical practice and the way that procedures are performed. Next, some background and discussion of the current state regarding the use of robotics in the operating room is presented. Finally, the topic of this dissertation is defined through discussion of the motivation, a statement of the problem, and a vision for the future.

\subsection{Background and Related work}

Modern surgical intervention can trace its history to the work of Ambrose Paré in 16th century France [1]; however, evidence of more primitive procedures and experiments have been found dating back several thousands of years earlier [2]. Since that time, the field has undergone extensive transformation as surgical knowledge, as well as technology, continues to improve. The majority of these advancements 
have ultimately led to improved efficiency, safety, and overall effectiveness while continuing to progress towards increasingly less invasive surgical techniques.

In terms of surgical procedures, there are typically three levels of invasiveness that describe a particular intervention. These consist of non-invasive, minimally invasive, and invasive arranged in order of escalating disruption to normal body function. The term non-invasive refers to procedures during which no perforation of tissue occurs and are typically of a diagnostic or therapeutic nature. This subset of surgical procedures will not be discussed in detail at this time.

\subsubsection{Open Surgery}

Open surgery is commonly considered an invasive procedure due to the use of a large incision to gain access to the body. Despite this fact, a significant number of surgical procedures are still performed using open surgery methods. The reasons for this are likely attributed to the difficulties associated with adapting complex procedures currently performed using open surgery. Translating these procedures to the methods of more desirable minimally invasive techniques such as laparoscopic surgery, single port access surgery, or natural orifice surgery requires significant effort due to the long learning curve necessary for surgeons to develop the required skills in these areas [3]. However, advancements in these areas are quickly allowing an ever increasing variety of procedures to be performed using one or more of these techniques [4].

\subsubsection{Laparoscopic Surgery}

Laparoscopic surgery is a form of minimally invasive surgery that can be traced back to Hans Christian Jacobaeus who performed the first such procedure on humans in the year 1910 [5]. This technique can be characterized through the 
use of small incisions in the abdomen of the patient in order to gain access to the peritoneal cavity, as shown in Fig. 1.1. These smaller incisions have the effects of reduced post-operative pain, shorter recovery time, and less visible scarring 6]. However, due to the use of small incisions, it becomes necessary to introduce some method of visualization which usually occurs through the use of a charge-coupled device (CCD) and fiber optics which allow an image to be collected at the distal end of the laparoscope and displayed on a monitor for the surgeon. Visualization in this manner limits the surgeon's perception and ultimately results in longer surgery times and potentially increased risk of surgical error [7, 8, 9]. These effects can be compounded by the fact that the surgeon also has limited dexterity when using laparoscopic tools [10]. The typical laparoscopic tool has four degrees of freedom (DOF) and requires the surgeon to operate using inverse motions due to the fulcrum effect at the location of tool insertion. Laparoscopic surgery would greatly benefit from the introduction of tools with greater freedom of motion and intuitive manipulation.

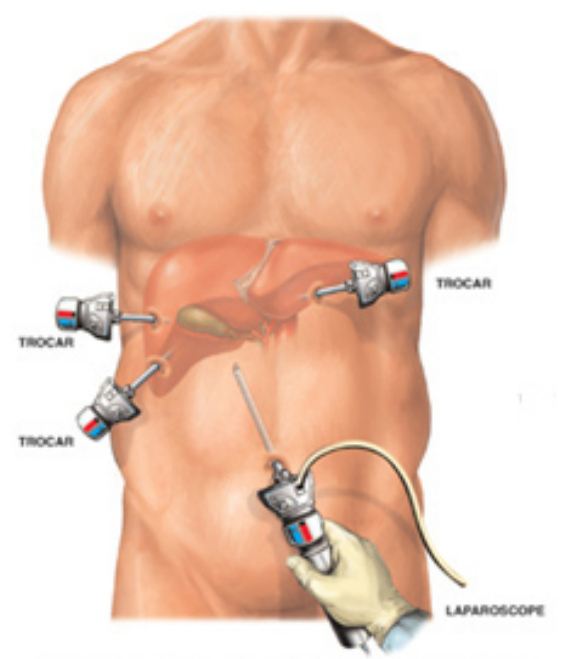

Figure 1.1: Example of laparoscopic surgery showing access ports and a visualization port [11]. 
Despite these perceived shortcomings, laparoscopic surgery has become a popular option for several routine surgical interventions. In particular, laparoscopic cholecystectomy, or removal of the gallbladder, has become one of the most wide spread laparoscopic procedures after being first presented by Dr. Reddick in 1989 [12]. Further, a panel representing the National Institutes of Health ruled that laparoscopic cholecystectomy was a suitable alternative to open cholecystectomy, while at the same time warning that the procedure necessitates surgeons of a high level of expertise [13]. This again is likely due to the counterintuitive input method. The use of proper surgical robotics would be expected to increase the speed at which a surgeon becomes proficient with this technique.

\subsubsection{Single Port Access Surgery}

Single port access (SPA) surgery, also known more recently as laparo-endoscopic single-site surgery (LESS), is similar in form to laparoscopic surgery with the distinction of using a single incision to gain access to the abdominal cavity (see Fig. 1.2), as the name suggests. Recent clinical trials suggest that SPA procedures are comparable to their laparoscopic counterparts in terms of operation time and rate of complications [14]. Further, SPA surgery carries the benefits of reduced scarring and possibly shorter hospital stays due to fewer incisions [15]. One reported downside to SPA procedures is a greater difficulty in performing complicated tasks due to limited triangulation; however, articulating instruments have proven beneficial and greater tool advancement would likely enhance the range of procedures that could be performed using a single port [14. Further, transitioning to an endoscopic or serpentine style surgical tool may also provide better performance in SPA surgery. 


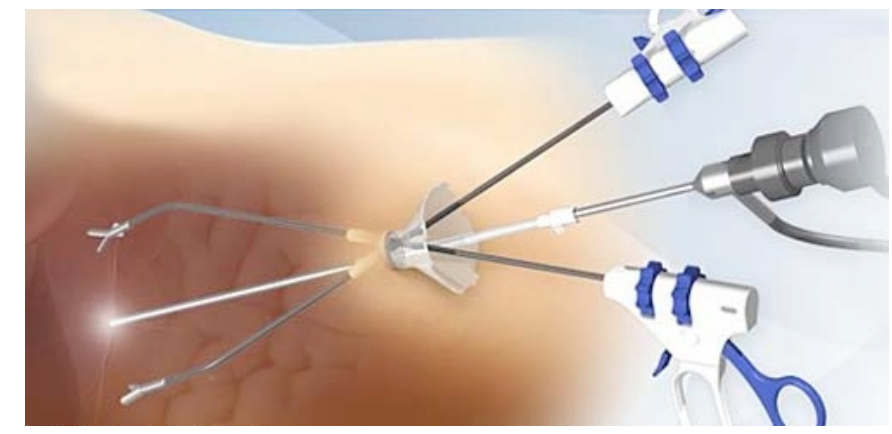

Figure 1.2: Example of single port access surgery [16].

\subsubsection{Natural Orifice Surgery}

Natural orifice surgery is a phrase used to describe surgery in which the location of interest within the patient is accessed through one of the body's natural orifices, such as the urethra, vagina, anus, or esophagus. More specifically, the phrase natural orifice translumenal endoscopic surgery (NOTES) is used to describe procedures during which an endoscope or similar tool crosses the gastrointestinal lumen to gain access to the peritoneal cavity as shown in Fig. 1.3. The first demonstration of a NOTES procedure took place in 1901 by Dr. Von Ott during which the female pelvic cavity was examined by inserting an endoscope through the posterior fornix of the vagina [17]. Since that time, steady progress has been made to bring NOTES to the state of development in which it exists today. Most notably, the work of Dr. Kalloo et al., published in full in 2004, is often credited as the first demonstration of the feasibility of oral, transgastric access to the peritoneal cavity in a porcine model [18, 19]. However, it should also be noted that Dr. Reddy and Dr. Rao have described performing NOTES procedures on humans in 2003 that went without publication [18]. Ultimately, the work of Dr. Kalloo et al. led to a joint effort between the American Society of Gastrointestinal Endoscopy (ASGE) and the Society of American Gastrointestinal and Endoscopic Surgeons (SAGES), which has defined the field of NOTES research and established a working group called the Natural Orifice Surgery Consortium for Assessment 
and Research (NOSCAR) in order to provide structure to the rapidly developing field of NOTES [18].

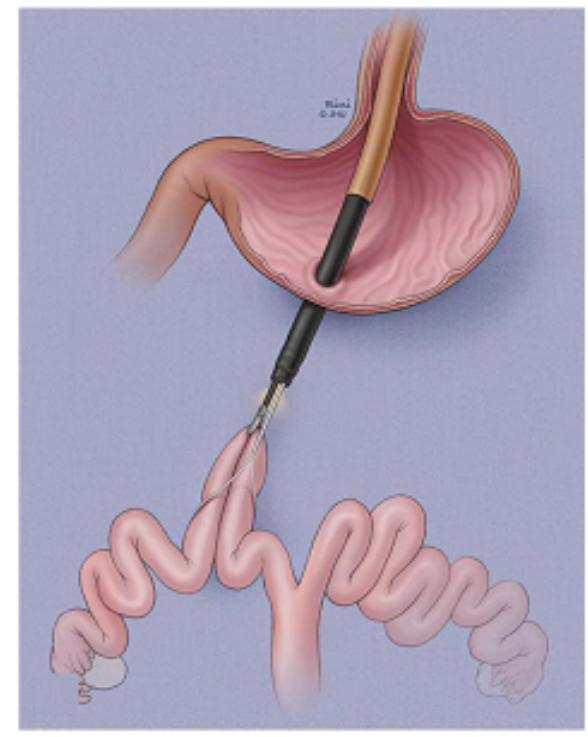

Figure 1.3: Example of transgastric natural orifice surgery [20].

The primary advantage of NOTES procedures is the reduced impact on patients, which includes no visible scarring, less post-operative pain, and decreased time to recovery [21. Further, analysis of a collection of 180 NOTES procedures found that the rate of complications was comparable with laparoscopy while there was a significant reduction in the use of medication for pain, post-operatively [22]. The importance behind these outcomes is significant as they have greatly contributed to the transition from open surgery, to laparoscopic surgery, to SPA surgery. Presently, the majority of NOTES clinical trials have been performed using traditional endoscopes adapted for the particular task; however, new advancements in specialized devices are becoming more common and will be discussed further in Section 1.1.5. 


\subsubsection{Robotics in Surgery}

The development and application of robotics for the operating room is a field that has undergone significant transformations in recent years as the push towards minimally invasive surgical techniques requires an increasing level of surgical precision and repeatability. Advancements in minimally invasive surgery have enabled robots to replace the camera-person, provide a more ergonomic environment for the surgeon, and even provide the means for a surgeon to perform a procedure from thousands of miles away [23]. Further, surgical robots can be employed as a tool for minimizing surgical errors such as those that may be caused by hand tremors and have been shown to improve the efficiency and speed of even novice surgeons [24].

The earliest recorded use of robotics in surgery can be found in the work of Shao et al. in 1985 when a robot designed for industrial applications was used to perform a neurosurgical biopsy [25, 26]. This work formed the foundation for a field that produced the first FDA approved surgical robot, the ROBODOC (Integrated Surgical Systems Inc.) for use in hip replacement surgery [27, 28]. More recent technologies in surgical robotics include the AESOP and Zeus robotic systems (Computer Motion) as well as the da Vinci Surgical System (Intuitive Surgical). After the merger of Computer Motion and Intuitive Surgical, the Zeus System was discontinued in favor of the da Vinci Surgical System. The da Vinci Surgical System consists of two primary pieces of equipment shown in Fig. 1.4. The first is a surgeon console which provides feedback from the robot to the surgeon and accepts input from the surgeon to control the robot. The second is the patient cart which includes a bed for the patient and the laparoscopic surgical instruments that perform the procedure. The tools of the da Vinci system are manipulated by an extensive arrangement of tensile wires. These wires run the length of the robotic arm and move in controlled sequences in order to articulate the tools in 
the desired manner. As can be seen, the system is rather large due to the technologies it employs and is prohibitively expensive for many surgical facilities. Despite this, the da Vinci Surgical System has become the standard in surgical robotics. However, as surgical techniques have advanced towards more difficult procedures and towards less invasive access methods, greater sophistication and ability is required of surgical robotics. Further, miniaturization of the robot would allow the robotic surgical system to be used in smaller surgical suites and possibly mobile environments.
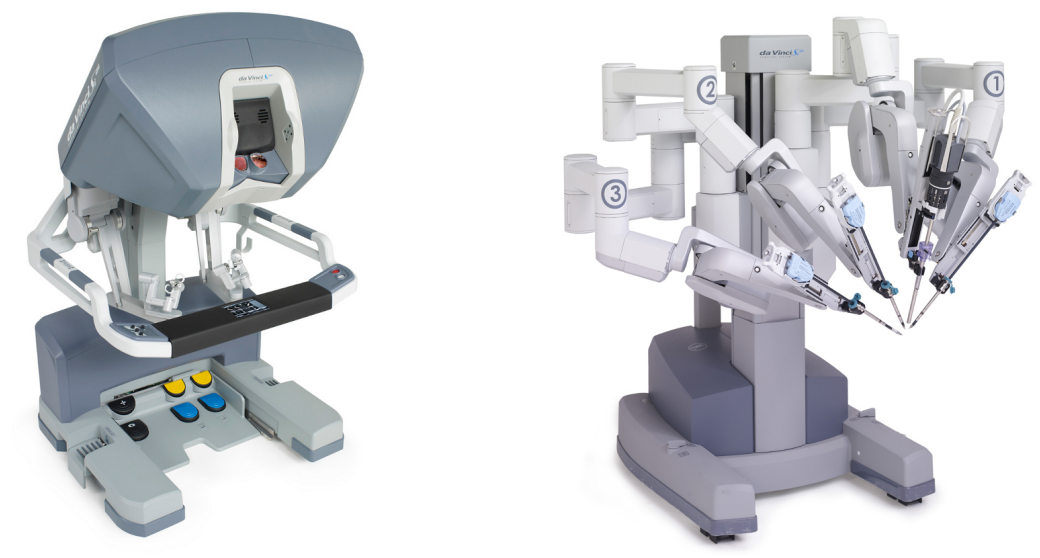

Figure 1.4: da Vinci Surgical System including surgeon console (left) and patient cart (right) [29].

The field of laparoscopic surgery has been particularly receptive to the use of surgical robotics. This has allowed the da Vinci Surgical System to become the only widely distributed, commercially available surgical robot currently on the market. Presently, there are more than 2000 da Vinci systems installed at hospitals around the world and thus the conversion of traditional laparoscopic procedures to robotic laparoscopy is occurring rapidly [30]. For example, the prostatectomy performed on the da Vinci system is becoming the standard treatment for prostate cancer [31]. This level of market penetration is helping to bring patient acceptance to the field of surgical robotics. However, as advanced surgical procedures transition towards a laparoscopic approach or other less invasive access methods, 
improvements are necessary both in terms of form factor and tool capability.

\section{Modular Robots}

The next realm of development for surgical robotics appears to be headed towards the even less invasive surgical access techniques such as single-port access or natural orifice access [32]. There are several emerging technologies related to surgical robotics that fall into broad categories ranging from modular robots to flexible endoscopic or serpentine robots [33. Beginning with modular robots (meaning that they consist of small standardized units that can be assembled or arranged), the work of Slatkin et al., shown in Fig. 1.5, describes the development of a robotic endoscope intended to gain access to the small intestine without the use of an external incision, something that was previously not achievable through the use of traditional endoscopes [34]. The device described therein employs pneumatic actuators to achieve various states of expansion and contraction to produce motion, similar to that of a segmented earthworm. Further, alternate segments of the device provide radial expansion which permits the device to achieve traction against the intestinal wall. Thus, by controlling both the radial and longitudinal expansion and contraction of the various segments, locomotion of the device through the intestines is possible. Experimental studies showed that the robot performed well even in situations involving flexible and slippery terrain. Although this device creates a basis for a whole new field of possibilities through surgical robotics, it provides imaging only with no tool capabilities.

Further developments in the area of modular robots are beginning to address the issues with positioning, manipulation, and teleoperation [33. For example, significant advances have been made in these areas using a remotely controlled single-port surgical robot known as SPRINT [35, 36]. The SPRINT robot is a master/slave system which consists of two independently manipulated arms intended to be introduced to the abdominal cavity through a single $3 \mathrm{~cm}$ access 
port located in the patient's navel. The joints of each arm are driven by DC motors (both external and embedded) and gears for each degree of freedom at the "shoulder", "elbow", and "wrist" joints. This device demonstrates the capabilities of these forms of robots in terms of dexterity and maneuverability by realizing 6 DOF per manipulator and a load capacity of $5 \mathrm{~N}$ [36]. While the geometric scale of this design is larger than the application presented here (maximum outer diameter of $18 \mathrm{~mm}$ ), it does show feasibility of using embedded robotic components. Lessons learned from the development of robots for SPA procedures can be used to inform the design of natural orifice robots.

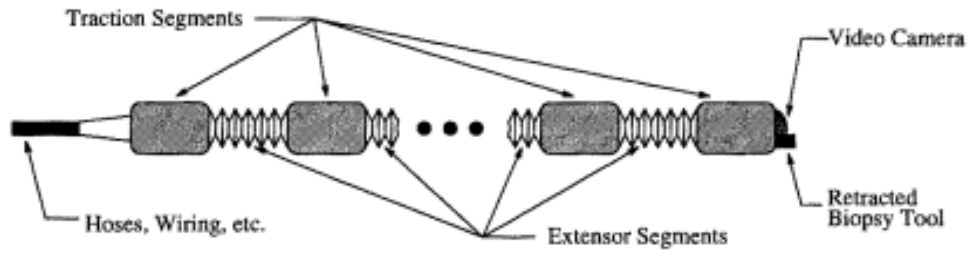

Figure 1.5: Endoscopic robot design described by Slatkin et al. [34.

While partially addressed by the work of Slatkin et al. [34, one constraint on novel surgical robots that attracts significant attention is the level to which the robot is connected to the outside world while deployed in the abdominal cavity. One method for addressing this issue is to use miniature in vivo robots [33, 37]. The complexity of these devices ranges from stationary or mobile devices with dedicated tasks such as visualization or lighting to robotic assistants that are capable of providing both visualization and tissue manipulation [6, 33]. Particularly interesting are a recent category of devices which make use of magnetic anchoring to physically isolate the device from the external world while maintaining the control over positioning and manipulation [38, 39, 40, 41. One possible realization of this concept is the use of multiple specialized robots deployed through the same access channel that work together to collectively provide visualization, lighting, grasping, cutting, and cautery [38, 39, 42]. Thus through the strategic placement of the various devices, procedures such as a cholecystectomy can be 
performed with no external incisions [38]. However, one disadvantage to this approach is that it introduces an extra layer of complexity to the surgical procedure since each of the various components must be managed independently. Another possible approach, is to use a more advanced in vivo robot that incorporates visualization and the necessary tools into one unit such as is described by Lehman et al. [41. This work presents an in vivo robot consisting of a main body housing lighting and visualization, as well as two arms located on either end of the body that provide grasping and cautery dissection. The device demonstrates promise for the future of advanced in vivo robots; however, issues with robustness of the device and insufficient tool maneuverability need to be addressed for such a device to be successful [41]. In particular, the use of rigid arm-like structures severely constrains the tools ability to manipulate safely within the abdominal cavity due to the necessity for large sweeping motions of the arms resulting in unintended collisions with abdominal tissue [43]. Further, the tool has the disadvantages of requiring assembly once inserted into the abdomen and using motion mechanisms that make its movements too slow for practical application.

\section{Serpentine-like Robots: Jointed}

Another approach is the use of endoscopic or serpentine-like robots which have advantages over modular robots in terms of large flexibility for minimal size [36]. Many of the clinical trials performed thus far in this direction have employed traditional endoscopes by passing the necessary tools through the scope's working channels. However, standard endoscopes are ill-equipped to provide the necessary freedom of tool manipulation and force capacity for many advanced abdominal procedures [33, 44, 45]. Therefore, novel endoscopic platforms are rapidly being developed to address the concerns of these advanced procedures. Robots of this variety take their inspiration from nature utilizing the concepts of biomimicry and bioinspired design [46, 47]. Many of these devices utilize a series of rigid joints 
driven by robotically manipulated cables to achieve tool positioning and manipulation such as is seen in the work of Phee et al. and others [48, 49, 50, 51]. Such devices are subject to several constraints that must be considered for them to be successful. These include the diameter of the instrument which should be approximately $20 \mathrm{~mm}$ if adapted to NOTES procedures, a minimum of two to three working channels, and flexibility in steering and control of position [33, 52]. These devices operate in a similar manner to the da Vinci robot previously described, while many of the same limitations remain such as tool manipulation and triangulation. This is particularly true in the application to transgastric NOTES, which often requires greater instrument flexibility than rigid laparoscopic tools can provide [53]. Phee et al. describe the design of jointed manipulators intended for use in endoscopically accessed abdominal procedures [48]. The robotic manipulators are controlled by externally located DC motors and long tendons. Each manipulator has 5 positioning DOF realized through the use of serially arranged joints and an overall diameter of approximately $10 \mathrm{~mm}$. Subsequent testing of this device revealed the ability to produce end loads in the range of $2.9 \mathrm{~N}$ to $5.2 \mathrm{~N}$ 49]. This device demonstrates significant capability for an endoscopically introduced manipulator but requires the use of externally located power components to achieve these results. The work of Kobayashi et al. [50] presents a surgical tool for SPA procedures which consists of an external positioning manipulator and internal tool manipulators. The overall tool has a diameter of $3 \mathrm{~cm}$ and the individual tools have diameters of $6 \mathrm{~mm}$ and $8 \mathrm{~mm}$. The positioning of the tools are externally controlled using rotating shafts and screws rather than long tendons thus avoiding the issues associated with long tendon manipulation such as sticking, slipping, and jerking [50]. Each tool has a bend range of $45^{\circ}$ which may be seen as a limitation in terms of the desired surgical workspace requirements. Finally, Shang et al. demonstrate the capability of a tendon driven robot to actuate through a large range of motion $\left( \pm 90^{\circ}\right.$ vertically and $\pm 225^{\circ}$ horizontally) by routing the tendons around universal joints to magnify the output motion [51, 54]. This design makes use of DC micromotors with a diameter of $4 \mathrm{~mm}$ to drive the 
universal joints. This has the benefit of enabling embedded actuation, but comes at the cost of load capacity being limited to $0.5 \mathrm{~N}$ at the tool tip and an overall outer diameter of $12.5 \mathrm{~mm}$. This design shows a lot of promise but currently has severe limitations on output load capability. Improvements in load capacity and greater miniaturization would make this device an attractive option for a surgical robot with embedded components.

\section{Serpentine-like Robots: Continuum}

Similar to the jointed, hyper-redundant robots presented in the previous section are a class of robots which are based on the use of a continuous flexible structure. Continuum manipulators have the advantage of improved simplicity relative to their jointed counterparts thus enabling greater miniaturization which is critical for many surgical applications as well as elimination of backlash that is present in jointed structures. On the small scale, the design of continuum manipulators is typically coupled with the use of tendons due to the geometric limitations along the length of the manipulator. One interesting example of a flexible serpentine robot design presented by Webster et al. is based on pre-curved, telescoping tubes [55]. The concept consists of tubes made of superelastic materials, such as nitinol, that are pre-curved in such a way that as they are extended from a straight semirigid outer tube the elastic energy stored within the material produces a known curvature. Subsequently, through the layering of multiple pre-curved tubes, the device can achieve complex shapes through variations in longitudinal and rotational positioning. However, since the device relies upon the strain energy stored within the pre-curved tubes in order to achieve manipulation passively, it is subject to severe limitations in situations where the application of a force is necessary. This introduces difficulties when it comes to procedures that require significant tissue manipulation. More recently this work has been adapted for use with pneumatic actuation in combination with the pre-curved concentric tube manipulators 
for surgical treatment of epilepsy [56, 57]. This development represents an interesting direction that may lead to greater capability for the pre-curved tube based manipulators and presents an interesting example of a fluid power approach. However, the current iteration includes large external frame to house the manipulation components. Further, the manipulator has a relatively short range and would require the access point to be located in close proximity to the surgical site thus making it difficult to apply this technology to endoscopic access techniques in its current form.

As previously mentioned, many of the upcoming designs in surgical robotics feature cable or tendon style inputs to drive the end effector. The ViaCath System (EndoVia Medical) presented by Abbot et al. [45] consists of two 6 DOF manipulators which are externally manipulated by servomotors and introduced to the abdominal cavity endoscopically. Each manipulator is approximately $5 \mathrm{~mm}$ in diameter. In experimental analysis the tool was found to be capable in terms of positioning and range of motion but was limited to $0.5 \mathrm{~N}$ of load at the distal end which was determined to be insufficient for most procedures. A secondary tool design was also presented which consisted of a jointed structure of $7.2 \mathrm{~mm}$ diameter, similar to the robots presented in the previous section. The second design demonstrated greater load capacity but was subject to the need for additional controlled inputs to manipulate the given joints. More recent work in this area has demonstrated that miniaturization of the continuous backbone style manipulator is possible and has been demonstrated down to a diameter of $4.2 \mathrm{~mm}$ [58, 59]. The distal manipulators of the presented design each have 4 DOF and are manipulated by an externally located actuation unit. Testing of the prototype revealed uncertainties due to the use of long tendons which extended from the distal manipulators back to the external actuation units. Each manipulator was shown to be capable of bending $90^{\circ}$ for the proximal segment and $60^{\circ}$ for the distal segment meaning that the manipulator as a whole could achieve a bending range of $150^{\circ}$. However, the tool load capacity is only $1 \mathrm{~N}$ at the tip which may 
not meet the requirements of common surgical procedures (see Section 2.1). Ding et al. [60] and $\mathrm{Xu}$ et al. [61] both present extensions of this design which use the same tendon style control to operate two manipulators and a vision unit on the same system which can be deployed endoscopically. This design features the ability to fold each of the three parts together for insertion and then deploy them for the procedure. The manipulators are then controlled via external actuation units similar to the previous design. Another feature of this design is an innovative method for suturing tissues through the use of pre-curved nitinol needles based on the work of Webster et al. [55]. This approach may alleviate part of the need for higher tool force capability. However, it severely limits the adaptability of the tool during a procedure by having a suture needle of specific size that cannot make use of existing suture needles already available in the operating room. Further, each of these designs requires a large external actuation unit to control the device which limits portability and introduces the potential for lag and inconsistency when controlling the unit.

In addition to the issues surrounding miniaturization, another major challenge faced by designers of continuum robots is that of producing accurate models of the robot's performance or behavior. These models are necessary both to facilitate a better understanding of the device as well as to enable design of a controller for the device. Early models were developed under the assumptions of hyper-redundant, rigid link based devices [62, 63]. While these models are quite effective, they do not account for the specific behavior of continuum devices present due to the existence of an infinite number of degrees of freedom [64]. Much work has been performed to model the behavior of the continuum manipulator using a variety of methods [65, 66, 67, 68. While these models provide great insight into the behavior of the continuum manipulator, they often require significant computational effort to arrive at a solution using numerical simulation. However, in the case of a low inertia system it is often possible to simplify the given models in such a way as to allow for more real-time calculation or simulation efforts for prediction of dynamic 
behavior. The desire to develop models with greater accuracy and less complexity is an ongoing effort and the best solution to this problem continues to evolve [64, 69].

Ultimately, robotic surgery presents several possible benefits over manual surgical techniques. As previously mentioned, robotic control of a surgical device introduces the possibility of reducing surgical error through cancellation of hand tremors or other methods such as image recognition [24]. Further, robotic surgical instruments have been shown through clinical studies to improve surgical efficiency, which translates to greater patient throughput and cost savings when compared with traditional procedures [32]. Beyond these benefits, other possibilities for an improved surgical experience include force feedback and telesurgery. Force feedback is one component of a surgical procedure that is often missing in robotic surgery. However, it has been shown that force feedback provides important information to the surgeon that can help to prevent tissue damage or other unintentional errors [70, 71, 72]. Therefore, the inclusion of force feedback in any robotic surgical instrument should be given strong consideration. Another benefit of robotic surgery is the potential for teleoperation. As discussed in the literature, teleoperation permits surgeons to operate from a more ergonomic position, which reduces fatigue and allows the surgeon to be located at a great distance from the operating room [23]. This in turn opens up a number of possibilities, such as surgical practice in under-developed countries where surgeons may be in high demand. Further, by operating the tools robotically, the task can be performed in a way that is more intuitive for the surgeon, with more natural kinematics. With all of these potential benefits, development in the field of surgical robotics is likely to continue at a rapid pace. Through the development of a surgical robot for MIS, it is possible to enable these types of advancements to better equip surgeons as they perform complicated tasks. The advent of endoscopic robots for performing surgical procedures enables such developments as natural orifice access techniques through inherent flexibility and maneuverability. However, even though 
endoscopic instruments provide greater flexibility, they are still hindered by limited stability while performing delicate procedures. Swanstrom et al. present a possible solution to the issue of scope rigidity [73]. The device uses ShapeLock technology which allows the scope to be oriented in the correct surgical position and then locked into place through the application of a compressive force within the shaft. This platform provides a useful base from which flexible surgical instruments may conduct the procedure and presents the possibility of greater tool capability.

The designs discussion in this section have primarily achieved their design requirements either through the use of external actuation units and long tendons which introduce uncertainty and a larger operating room space requirement or by increasing the size of the manipulators to accommodate embedded components. While each of the designs presented here show promise, there remains the need for greater capability at a smaller scale to enable the design of robotic surgical manipulators which can be embedded within the working channels of existing endoscopic instruments.

\subsection{Motivation}

The use of robotics in surgery is becoming increasingly necessary with the advent of new minimally invasive surgical techniques such as single port access (SPA) surgery, a single incision procedure during which access to the abdominal cavity is typically gained through the navel, and natural orifice translumenal endoscopic surgery (NOTES), an experimental technique that involves performing traditional surgical procedures with a flexible endoscope through a natural orifice such as the mouth, rectum, or vagina. These sorts of novel techniques remove the need to create incisions across the abdominal wall, thus minimizing or even eliminating 
post-operative pain, abdominal scars, and complications such as incisional hernias. Other potential benefits include reducing postoperative recovery time, using moderate or deep sedation instead of general anesthesia, and the potential to perform many surgical procedures outside of traditional operating rooms. Because of these advantages, techniques such as SPA surgery and NOTES appear to be the next natural step in the progression of minimally invasive surgery for routine procedures such as cholecystectomies and exploratory laparoscopies in hospitals around the world [18]. Due to the complexity of these procedures and the natural limitations of space and site access, the development of advanced robotics becomes imperative for the future success of these sort of surgical interventions.

One approach to the development of surgical robots is fluid power, which can come in the form of pneumatic or hydraulic. In particular, hydraulic power advantageously has higher power density (on a per weight or per volume basis) on the order of 10:1 or greater when compared with other driving methods [74, 75]. Additionally, a hydraulic operating fluid such as saline would provide less uncertainty due to compressibility than a pneumatic fluid and thus enable greater precision. However, the underlying challenges surrounding this assumption include a question of what limitations exist on the miniature scale for which the benefits of a hydraulic approach hold true. For example, work is currently in progress exploring the use of fluid power at the meso-scale ( $\mathrm{mm}$ to $\mathrm{cm}$ ) for use in prosthetics [76]. The next logical step is to explore the use of fluid power at the micro-scale ( $\mu \mathrm{m}$ to $\mathrm{mm}$ ) for which the research is ongoing in the field known as microfluidics [77, 78]. However, as pointed out by Love et al., there exists a gap between conventional fluidics and microfluidics that includes the type of high pressure-low flow rate fluid power components that would be necessary for the application of hydraulic power to the field of surgical robotics [76]. Therefore, it is necessary to conceptualize the methods by which these sorts of fluid power components might be realized.

For surgical robots to adapt to these developments, it is necessary to overcome significant challenges in terms of tool manipulability and precision. By embedding 
the robotic components close to the manipulators, it is possible to reduce the uncertainty present within the system while achieving similar tool capability. Most systems rely on a large external robot manipulating cable driven tools which inherently result in uncertainty due to the use of long tendons [58, 79]. Due to advantages in power density and relative incompressibility [75], hydraulics may allow surgical robotics to be embedded endoscopically. Yet, the use of hydraulics in this field has been under-explored due to difficulties associated with the integration of hydraulic components into the distal end of the robotic manipulator due to size constraints [80]. The few attempts at using hydraulics for surgical robotics have typically been limited to external manipulation of the surgical tools rather than an embedded design [81, 82].

\subsection{Problem Description}

There exists a wide range of abdominal procedures for which a robotic surgical tool may be employed. Access to the abdominal cavity may be gained through abdominal incisions, the patient's navel, or natural orifices such as the mouth, vagina, or rectum. In the case of oral access, the surgical tool deployed via an endoscope which in turn would be passed through an incision in the stomach wall and into the abdominal cavity. From this transgastric access point, the surgeon could perform the necessary tasks ranging from gallstone removal to gastric bypass surgery. The robot as a surgical tool provides the surgeon with the ability to perform internal procedures without direct line of sight or direct external access to the surgical site. The greatest difficulties faced when applying robotics to these types of procedures come in the form of severe constraints on the design in terms of force requirements, range of motion requirements, and limited available space. When examining the variety of surgical robots in the literature, it is apparent that there is a trade-off between size and functionality. The goal of this work was to demonstrate that hydraulic power can be employed advantageously in the design 
of a robotic surgical tool for minimally invasive procedures and enable the use of embedded robotic components to achieve a smaller overall footprint within the surgical suite. Size constraints for endoscopic surgical devices are dictated by the geometric constraints of the patient's anatomy. The outside shaft diameter for the device is typically limited to a range of 18 to 22 millimeters based the average size of the patient's esophagus [52]. Contained within this diameter, it is necessary to include a channel for imaging and at least two tool channels, as well as additional ports for insufflation, suction, and irrigation. Considering these requirements, a practical limit on the tool channel diameter of 5 to $6 \mathrm{~mm}$ is typical. It is within this available space that the mechanisms for both device articulation and tool manipulation must be contained. Further, the surgical instrument needs to be capable of $180^{\circ}$ retroflexion in order to navigate the complex abdominal anatomy [52]. This requirement is particularly important for oral access techniques since it is not possible to reach the upper quadrants of the abdomen without retroflexion. Looking at the force capabilities required from the tool, it is important to consider the forces necessary for both gross tissue manipulation and common procedural applications such as suturing. Through experimental investigation it was determined that a load capability of approximately $5 \mathrm{~N}$ would provide sufficient tool capability. The lack of a clear solution for a robotic device that meets these requirements provided the impetus to pursue a novel design.

To achieve these goals, it is necessary to consider each of the various subcomponents that comprise the assembled surgical tool and the interactions between them. These components provide the mechanics of tool positioning through the support structure, actuation unit, and method of control input. First, the structure of the tool which provides support and shape needs to be determined and modeled. Next, it is necessary to determine the method by which the tool is manipulated through the use of an actuator or motor. It is then necessary to specify the control method for the device. Finally, the interactions between the individual subcomponents and the system as a whole needs to be analyzed and understood 
in order to evaluate how the tool interacts with its environment.

\subsection{Vision}

The work described here intends to identify the limitations of existing robotic surgical platforms and develop a means of overcoming them while satisfying the tool requirements of force output and manipulability. Currently available surgical robots are developing rapidly, but a technological gap exists in the area of small scale, high capability platforms. It is hypothesized here that this gap can be filled by taking advantage of the power density and other properties of fluid power, such as incompressibility and efficiency; thus permitting surgical robots to meet the needs of advanced minimally invasive procedures. The approach discussed here differs significantly from conventional surgical robots in that the robot is embedded within the surgical device itself (see Fig. 1.6); whereas in the conventional system, a general-purpose robot is used to manipulate various surgical tools. The advantages of this approach are that with proper technologies, the robotic manipulator can exhibit greater freedom of motion, have better controllability, be more cost effective, and have superior ease of mobility. The improved mobility combined with relaxed requirements for operating room cleanliness can be potentially useful for mobile clinics, out-patient clinical settings, and on the battle field. Being more cost effective and having a small overall size, the envisioned robotic assisted surgical devices can be widely deployed, even in rural or other less technology intensive environments. Hydraulic actuation typically displays greater stiffness and faster response time than the comparable electromechanical or pneumatic counterparts. Further, due to their ability to operate at low velocities with high forces and the lack of an upper limit on power input, hydraulic systems can achieve greater power density which allows for miniaturization of the device components [83]. When compared with a pneumatic approach, hydraulic power stores less energy in the form of compressed fluid. Thus hydraulic power 
can be safely operated at a higher pressure than a similar pneumatic system. Further, if a compatible hydraulic fluid is selected, such as saline, leakage is not likely to result in contamination of the abdominal cavity. Lastly, the low costs of the components used in this design would permit the device to be disposable and thus loosen requirements for sterilization.

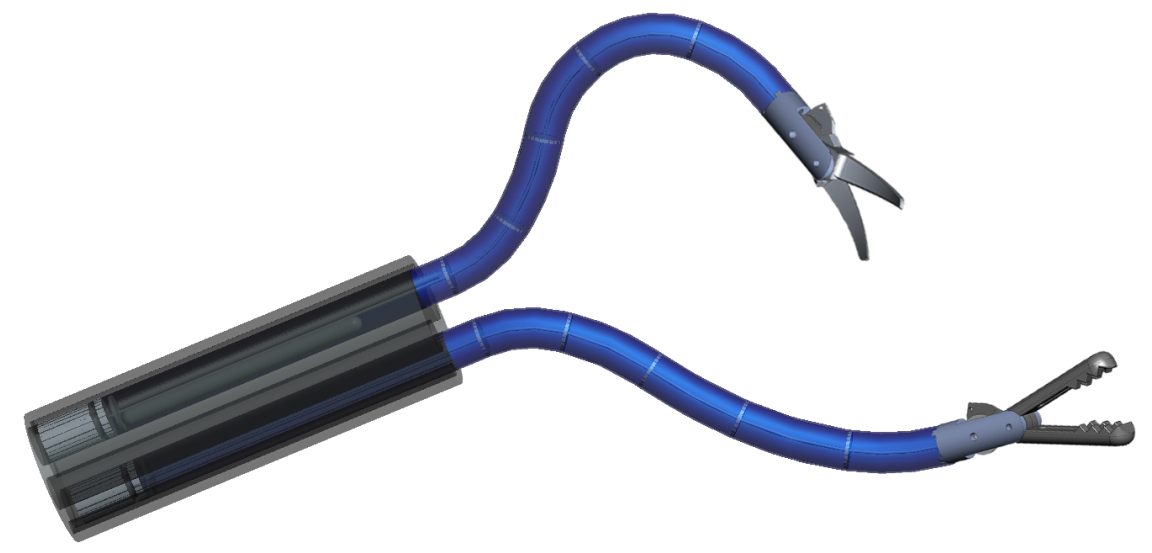

Figure 1.6: Envisioned design of the robotic surgical tool for natural orifice surgery. Shown with example end effector tools including a grasper and a scissors [84].

Through the development of a novel surgical manipulator, I seek to show that the use of fluid power in this application is an achievable goal and would allow for greater miniaturization while maintaining or improving robotic capability. The surgical robotic platform described here consists of three basic units: manipulators, actuators, and control valves. All components are designed to be located in the distal end of the endoscope and contained within its working channel with a diameter of approximately $6 \mathrm{~mm}$. Figure 1.7 shows a simplified version with two manipulators. These manipulators would serve as a platform for the end effector tools (see example tools in Fig. 1.6), which could include graspers, scalpels, scissors, or clip applicators, each manipulated by its own actuator [85]. These end effectors could be deployed individually with the manipulator or stored distally within a tool holder. Each manipulator can be divided into multiple serially connected segments with a single flexible backbone and is actuated by a set of 
tendons/wires, similar to other serpentine-like robots [86, 87, 88]. The bending of each manipulator segment is controlled by a group of three tendons antagonistically operated by hydraulic artificial muscle actuators at the base of the platform. The tendons, which are arranged circumferentially, pass through intermediate discs and terminate at the end of the given segment. The tension in each tendon controls the bending of the segment in one direction. Unlike cable driven surgical tools that are actuated via long cables connected to external motors or actuators, in this design the tendons are kept relatively short since the muscle actuators are small enough to be stacked serially and placed distally within the lumen of the endoscope. Hydraulic flow/pressure to the muscle actuators are modulated by valves which are also contained within the endoscope behind the muscle actuators. Thus the only connections required externally are lines for the hydraulic supply (assumed to be a constant pressure supply) and return as well as the electronic signal wires. Figure 1.8 shows the basic structure of both a singlesegment manipulator (Fig. 1.8a) and a multi-segment manipulator (Fig. 1.8b). Each version is shown with only a minimum of included components and is not meant to illustrate the full system assembly.

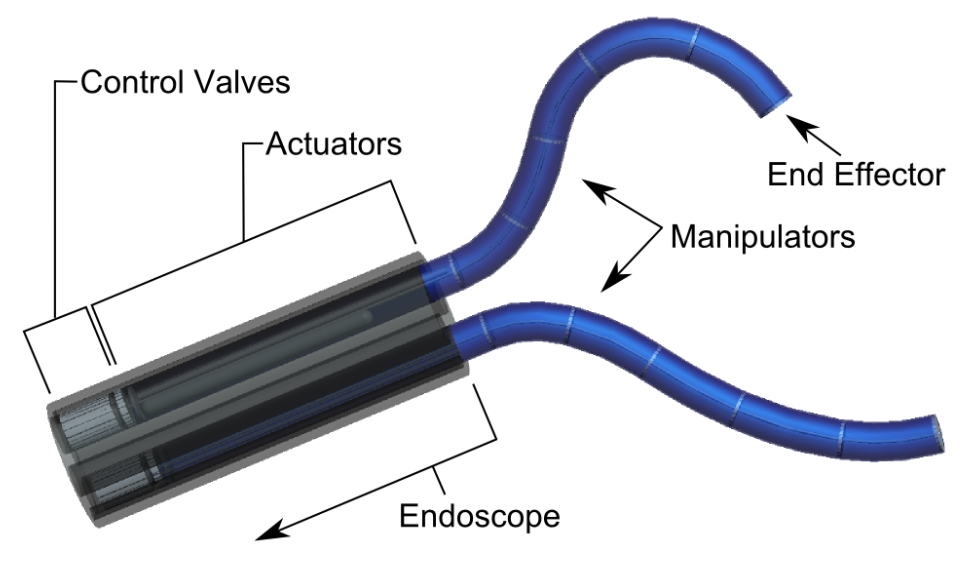

Figure 1.7: Model of the robotic surgical tool design shown with two manipulators, each with two individual segments.

The usage scenario for this device would be similar to the scenario used by many 


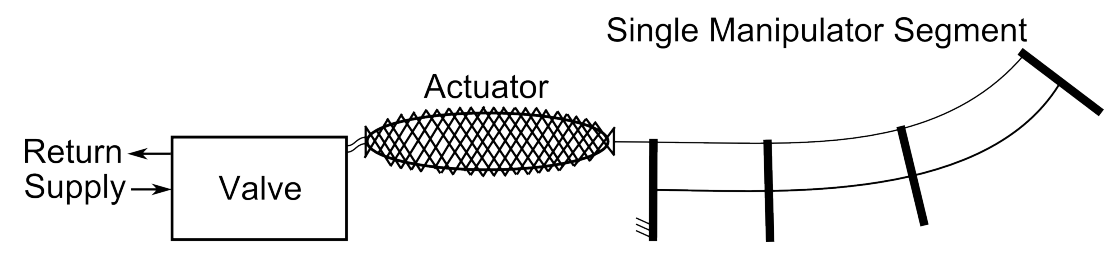

(a)

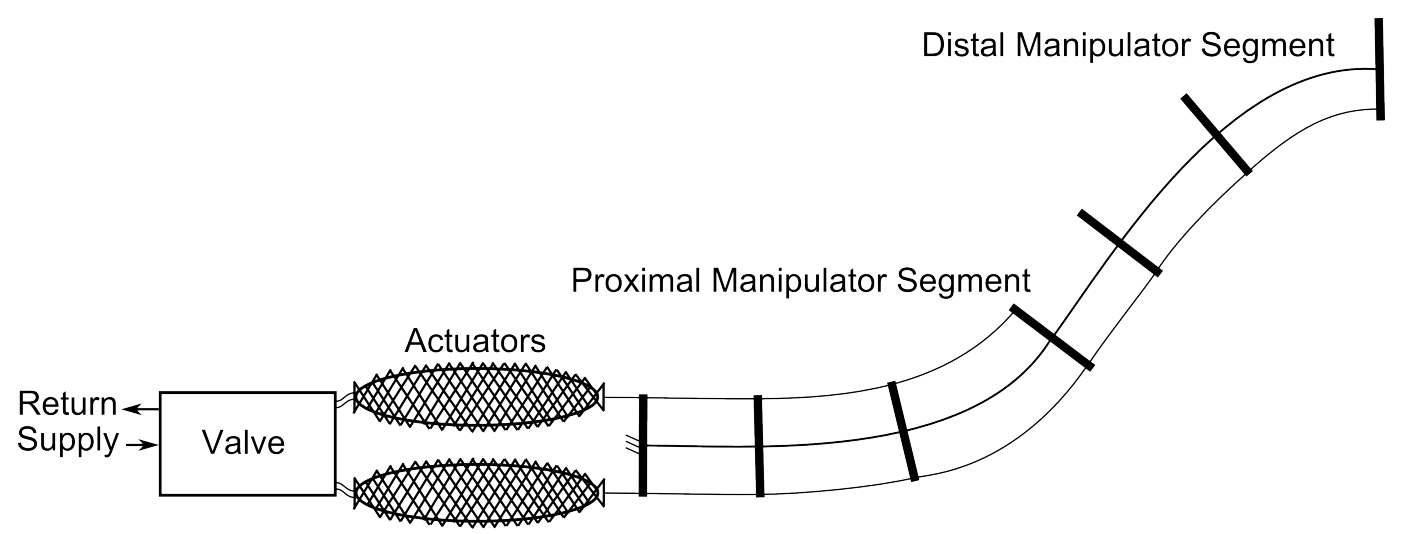

(b)

Figure 1.8: Line drawings showing a minimal version of how the valve, actuator, and tendon components are connected for (a) a single-segment manipulator and (b) a multi-segment manipulator.

similar robotic systems discussed in Section 1.1.5. In practice, this system would consist of an easily deployable bedside cart with a hydraulic supply. Surgical input would be provided either on-location or remotely from a surgeon input console. The device components are inexpensive enough to be disposable but are sealed such that sterilization is possible when necessary. Once in the surgical suite, preparation of the patient would involve gaining access to the abdominal cavity using the methods currently being developed in the field of NOTES (Section 1.1.4). Once access is achieved, the endoscope would be positioned near the area or organ of interest such as the gallbladder or small intestine. The endoscope would then be rigidly locked in place using a technology such as ShapeLock [73]. From this platform the robotic instruments presented here would be deployed (see Fig. 1.9). The surgeon as the operator would manipulate the robot from a console similar to what is employed by the da Vinci Surgical System (see Section 
1.1.5). This console would allow the surgeon to control multiple segments per manipulator in a manner that mimics natural body motion by measuring multiple joint orientations and mapping them each to the actuators for each manipulator segment, for a total of three DOF per manipulator segment. A stereoscopic video feed would be streamed to the console from the distal end of the robot using fiber optics. The manipulators allow the surgeon to remotely perform all of the necessary common surgical tasks such as suturing, tissue manipulation, or tissue resection. Upon completion of the procedure, the manipulators are retracted from the endoscope to allow for closure of the access site. The relatively low cost of the manipulator components would allow the manipulators to be discarded after use. In the subsequent pages I intend to demonstrate that this vision of greater miniaturization while maintaining or improving robotic capability can be achieved through the application of fluid power as an enabling technology. 


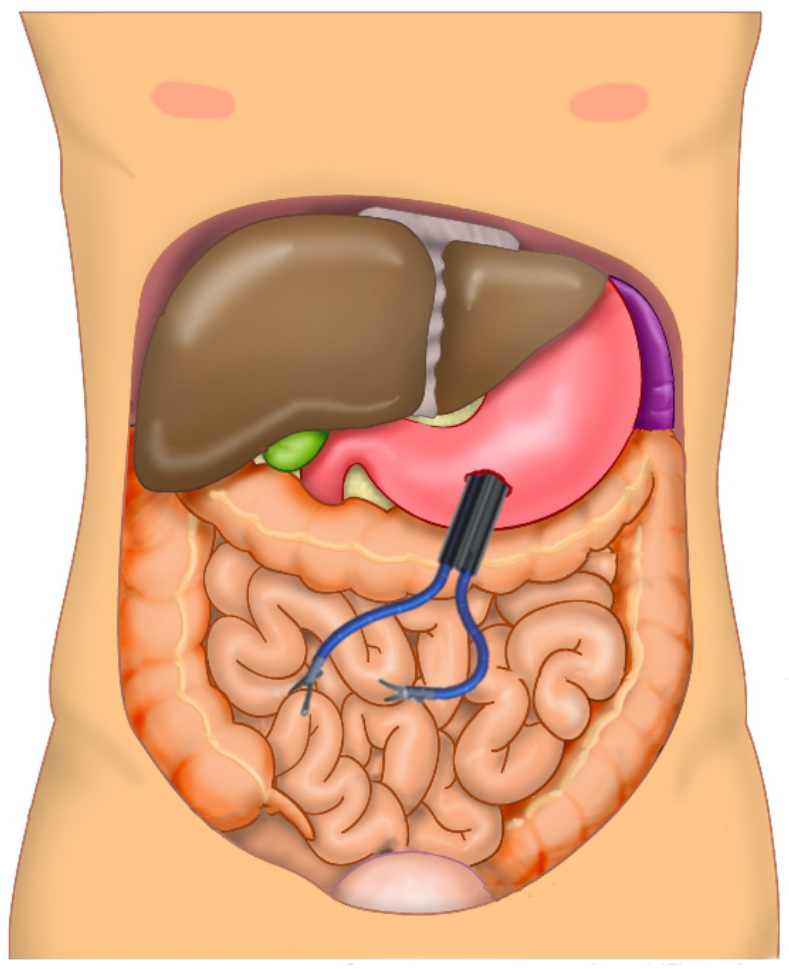

Figure 1.9: Illustration of the robotic design during a procedure showing access to the abdominal cavity gained through an incision in the stomach wall.

\subsection{Dissertation Overview and Contributions}

This introductory chapter presented a brief history of the evolution of surgical intervention as well as an overview of recent developments surrounding the application of robotics to the field. While the range of approaches and technologies being adapted for use in minimally invasive surgery is vast, it is clear that there exists a need for a small scale, highly capable surgical manipulator. A vision for the design of a surgical manipulator was presented that takes advantage of the properties of fluid power to meet the needs of emerging surgical procedures.

Through the efforts presented in this dissertation, much new information was produced and developments resulted. The use of hydraulic power for surgical robots 
has not been extensively explored and thus the considerations unique to this approach which have been evaluated are valuable and applicable to other surgical tasks where hydraulic power may be used advantageously. A quantification of the load requirements for surgical robots performing abdominal procedures was produced which will provide a guide for other researchers developing surgical robots. These values have typically been reported anecdotally in the literature and thus are a valuable resource for the field. An alternative model for predicting the behavior of continuum beams under load was developed to provide an inverse formulation for computing beam shape and end loads. This is useful as continuum beams are widely being explored for use as minimally invasive surgical manipulators as well as in a wide variety of other applications. Finally, a novel valve concept and two possible designs realizing this concept were developed. These valve designs facilitate control over the three actuators in an antagonistic arrangement. Further, the valve designs enable proportional control of the three actuators at a size scale not commercially available. The design of a novel hydraulic surgical manipulator as a summation of its parts has been described. This device builds upon the variety of approaches found in the literature while adding several useful contributions such as enhanced miniaturization and internally contained components.

In Chapter 2, the design requirements for a surgical robot designed for use in natural orifice transgastric endoscopic surgeries are described. These requirements include considerations for the size of the surgical workspace, safety and biocompatibility, and load capacity of the tool. Due to a lack of tool force requirement quantifications in the literature, a set of experiments were carried out for expected high force usage scenarios to support robotic manipulator design through an evaluation of tool force requirements. The results presented allow for performance analysis of existing surgical tools as well as inform future directions for similar devices.

Chapter 3 provides a detailed description and analysis of the selected backbone 
structure. An alternative model using the principle of minimum potential energy and inverse kinematics is presented to predict the behavior of the backbone. The model is then experimentally validated to demonstrate that it is capable of producing accurate beam shape predictions under most circumstances. Recommendations are provided as to how to best utilize the model under the prescribed assumptions.

In Chapter 4, the selection of an actuator to drive the manipulator is presented. The performance of the actuator is predicted analytically and confirmed experimentally. Further, an optimization algorithm is used to determine the ideal parameters necessary for fabrication of an actuator which maximizes range of motion within the specified constraints placed on actuator diameter and force capability. This was accomplished through modeling of the stresses present within the actuator using finite element analysis. It is demonstrated that the requirements placed upon the actuator can be met using the given design.

Chapter 5 describes a method of providing control input to the system by way of a control valve. To meet the needs of the presented device within the constraints of the application, a novel valve design is conceived. Design parameters that are necessary to achieve desired valve performance were calculated. Two realizations of the design were fabricated at a larger scale to provide a means of evaluating the design performance and confirming the hydraulic principles used to develop the design. The results presented suggest that the design can be scaled to a size appropriate for application to the field of minimally invasive surgery.

In Chapter 6, a description of the assembly of the novel manipulator is provided. A model is shown which combines each of the manipulator components to allow for prediction of manipulator performance. Design specifications are given for each of the components that make up the assembly such that the design is theoretically capable of meeting the requirements set forth in Chapter 2. Additionally, results from testing of a manually operated manipulator assembly are shown to 
demonstrate efficacy of the design. It is also demonstrated that it was possible to achieve the desired performance objectives using this design. 


\section{Chapter 2}

\section{Design Requirements}

The first task in any design problem is to lay out both the constraints and requirements that help direct the design process towards a workable solution. This chapter attempts to do that by identifying the important considerations rele-

vant to the design of a robotic manipulator for minimally invasive surgery and then quantifying those requirements using both experimental measurement and information from the literature as appropriate. Consideration is given to the load capacity required of the tool, the size of the workspace, and issues involving safety and biocompatibility. Within each of these areas, recommendations are provided to help guide the design of the surgical manipulator.

First, in Section 2.1 the force requirements for a surgical manipulator for use in abdominal procedures are experimentally quantified. This is followed by a discussion of the tool requirements in terms of the workspace, safety considerations, and biological constraints in Sections 2.2, 2.3, and 2.4, respectively. Finally, the requirements discussed in these sections are summarized in Section 2.5 . 


\subsection{Tool Force Requirements}

\subsubsection{Motivation}

Surgical robots have allowed surgeons to perform a variety of procedures using less invasive techniques. These new techniques make it possible to minimize or even eliminate post-operative pain, scarring, or other complications. Further, the advent of robotic surgical tools allow for the inclusion of technologies such as force-feedback to better inform the surgeon and enable greater performance [89].

It has been shown that expert surgeons exert less force on the tissue when compared with novice surgeons. However, it is important to understand the forces necessary to perform a particular surgical task for both the proper design of surgical tools and to minimize the potential for tissue damage [90, 91, 92. Through experimental evaluation of these surgical tasks, it is possible to develop a better understanding of the force limitations and better define the tool force requirements. In this section, the force requirements for a surgical manipulator operating within the abdominal cavity will be quantified.

Previous work in this area has quantified surgical forces in terms of both suture knot holding strength and suture tightening, as well as in the quantification of the forces associated with tissue puncture with a scalpel blade or needle [93, 94, 95, 96, 97, 98, 99]. Additionally, the abdominal organ tissues have been characterized in vitro with rectangular samples [100]. However, these studies have limited applicability to clinical applications and the results of these studies show a wide range of variability in the measured forces due to the selected organ, the methodology, and the inherent nonlinear biomechanical properties of soft biological tissues. Thus, a better understanding of the forces associated with specific surgical tasks is necessary. Particularly, the forces associated with suturing, including suture needle tissue puncture and the force necessary to break the needle 
away from the suture line, have not been well characterized previously. In addition, little work has been done to quantify the forces necessary for manipulation of the abdominal organs. The general assertion is that the biological tissue should be handled in such a way to minimize the potential for tissue damage [101]. However, these forces need to be quantified to facilitate the design and development of surgical instruments.

Though there are a variety of tasks common to surgical procedures, manipulation of the abdominal organs and suturing were selected for evaluation of their force requirements as part of the present study. These tasks were selected due to their widespread applicability to a variety of surgical procedures and their likelihood of having greater tool force requirements. Further, in the case of suturing, the force capability of the surgical robot can affect the quality of the knot and its ability to perform as intended [93]. The results of this study will help to define the force requirements for novel surgical tools for procedures involving suturing tasks and the manipulation of soft biological tissues.

\subsubsection{Methods}

In order to determine the tool force requirements, a Nano25 six-axis force/torque transducer (ATI Industrial Automation) was mounted between a handle and probing tip to provide an intuitive interface for force measurement that could be used in the operating room, as shown in Fig. 2.1. Through a collaboration with The Visible Heart Laboratory (University of Minnesota) [102], access to a cadaveric porcine model was possible, which enabled measurement of the forces necessary for abdominal tissue manipulation. Abdominal tissues including the liver, spleen, gallbladder, stomach, kidney, and bladder were examined by using a force probe to manipulate the tissue such as may be necessary during surgical intervention. The force was recorded as a magnitude applied to the tip of the probe. Then the peak value was selected from the data set for each organ and reported. In each 
case, the organ was manipulated in order to gain access to the underlying tissue or surrounding organs. Care was taken to avoid damaging the tissue by using the minimum force necessary to move the organ, in accordance with the practice of minimizing the potential for tissue damage as suggested in the literature [101]. As can be seen in Fig. 2.2, the abdomen was open during testing, which would be similar to the conditions during surgery when the abdomen is inflated.

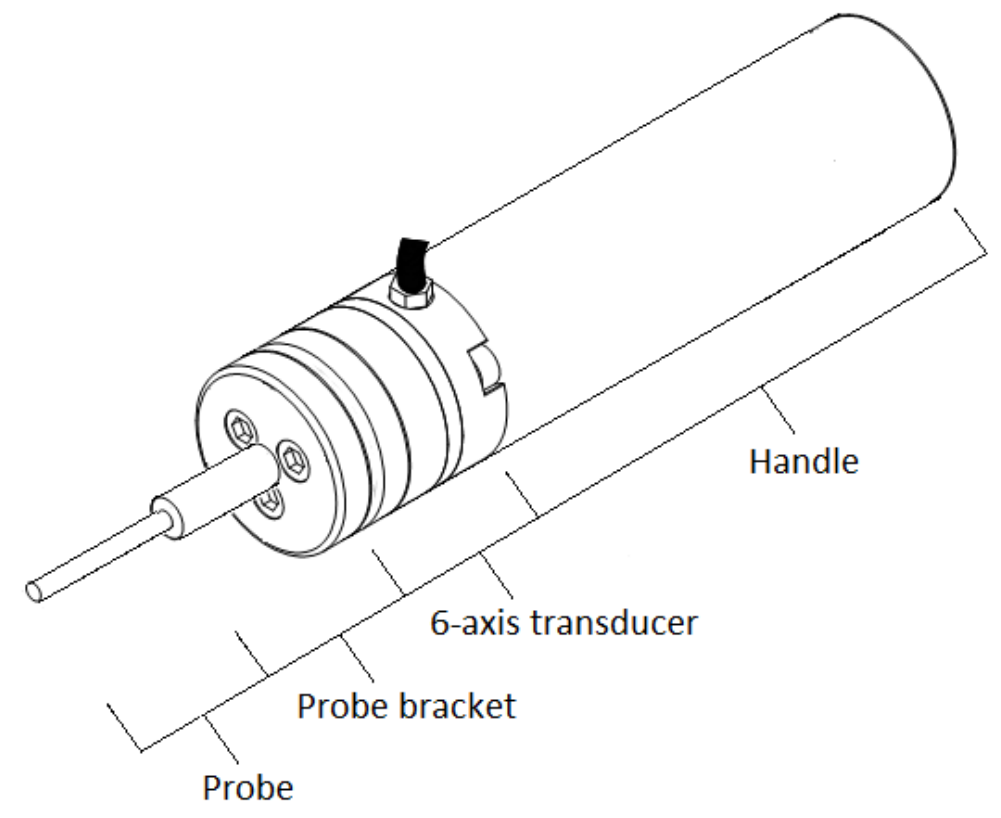

Figure 2.1: Illustration of the mounted six-axis transducer

Using the same instrumented force probe, results were also collected for common suturing tasks such as tissue puncture and knot tightening, seen in Fig. 2.3. where high tool force requirements would be expected. Both porcine liver and gallbladder tissues were used for needle puncture force measurements. The suture needle (size 4-0, 1.5 metric) was attached to the force probe shown in Fig. 2.1 and was passed through the tissue as would be done for a normal suturing procedure. The peak magnitude of the force on the tool tip was recorded.

Next, suture knot tightening forces were measured using both size 0 (3.5 metric) 


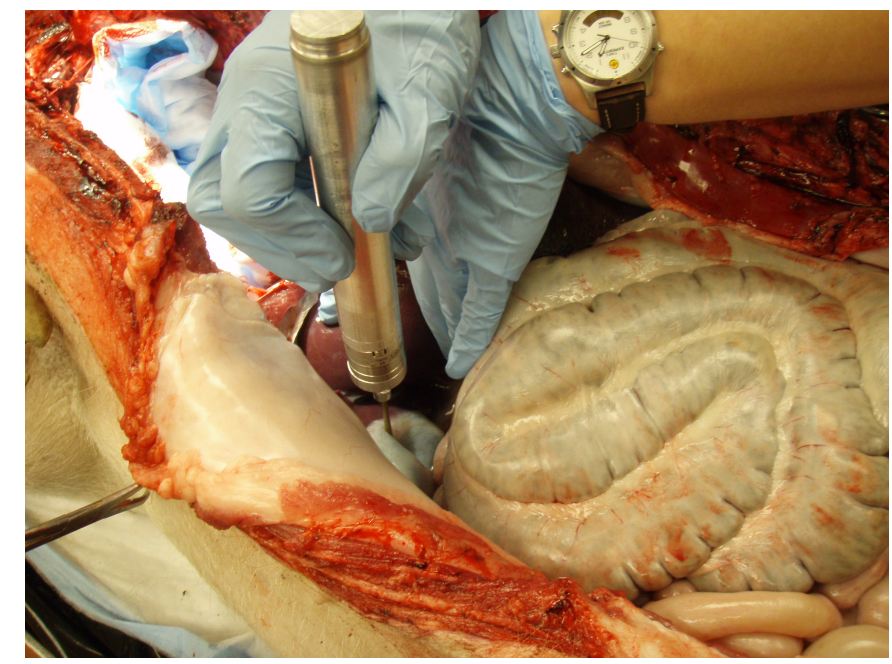

Figure 2.2: Force measurements being taken in a porcine abdomen

Table 2.1: Suture sizes and types evaluated for break force

\begin{tabular}{cclll}
\hline U.S.P. Suture Size & Metric Size & Material & Product & Manufacturer \\
\hline 1 & 4 & polybutester & Novafil & Davis \& Geck \\
$3-0$ & 2 & polypropylene & Surgilene & Davis \& Geck \\
$4-0$ & 2 & chromic gut & - & Davis \& Geck \\
$4-0$ & 1.5 & polypropylene & Surgilene & Davis \& Geck \\
$5-0$ & 1 & polypropylene & Surgilene & Davis \& Geck \\
$6-0$ & 0.7 & braided polyester & Polydek & Deknatel \\
$6-0$ & 0.7 & polypropylene & Surgilene & Davis \& Geck \\
$7-0$ & 0.5 & polypropylene & Surgilene & Davis \& Geck \\
\hline
\end{tabular}

and size 4-0 (1.5 metric) polypropylene (Surgilene, Davis \& Geck) sutures. The suture lines were pulled, and the tool tip force measured, until a satisfactory knot was formed, as shown in Fig. 2.3. This method introduces inherent variability between the individual surgeons tying the knot. However, by maintaining consistency within this study it is possible to compare the knot tightening forces between suture sizes.

Lastly, the forces necessary to break the suture line were quantified using a variety 


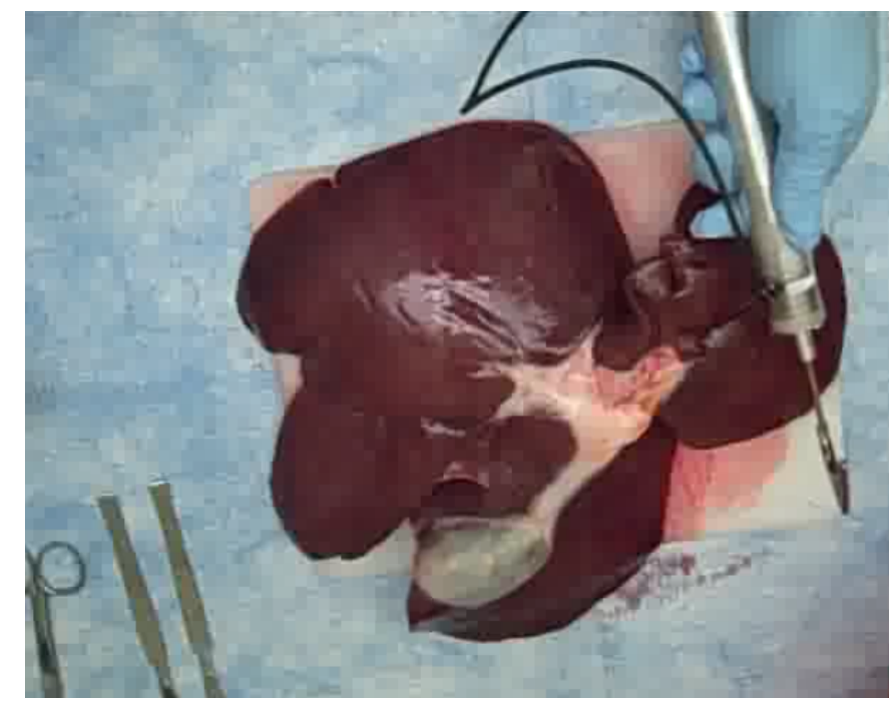

Figure 2.3: Evaluation of suture knot tightening force requirements

of suture sizes and materials as shown in Table 2.1. The suture needle was rigidly clamped as shown in Fig. 2.4, which was itself mounted to the crosshead of a tension testing machine. The free end of the suture was then secured to the bottom frame of the tension testing machine using a looped knot. Mounting the suture in this way made it possible to evaluate the suture break force regardless of the location of the failure, whether the break occurred in the suture line or at the junction between the line and the needle. The break force was measured using a 250 N, uniaxial load cell (Part Number 4501018/B) mounted on a Qtest QT/10 (MTS Systems Corporation) tension testing machine. Additionally, the crosshead of the tension testing machine was moved vertically at a rate of 0.1 meters per minute until breakage occurred. Displacement rates of 0.05 and 0.2 meters per minute were also tested but the dependence of the break force on displacement rate was found to be insignificant. 


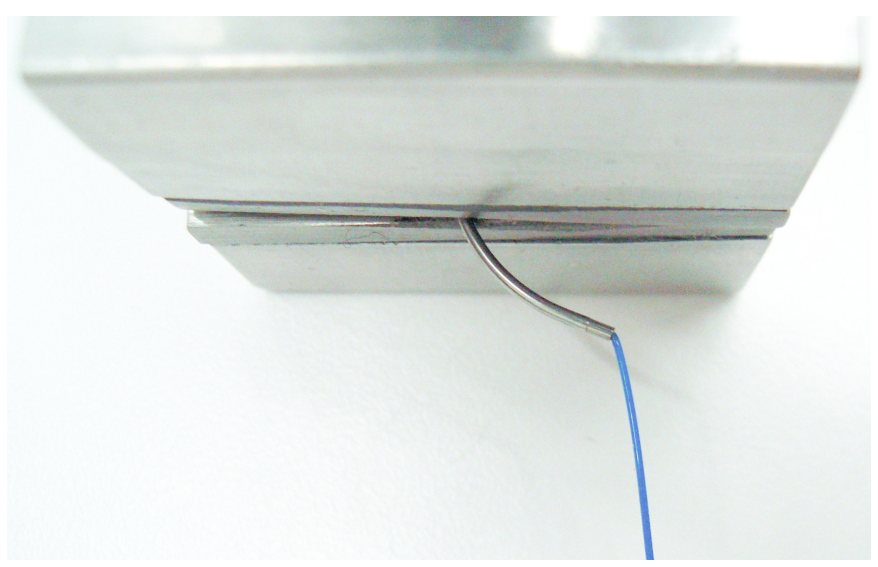

Figure 2.4: Rigidly mounted suture for measuring break force

\subsubsection{Results}

As can be seen in Table 2.2, the greatest forces recorded during the abdominal force probing experiments were for manipulation of the kidney and liver tissues, which each demonstrated a measured load of approximately $3.5 \mathrm{~N}$. This result would be expected due to the rigidity of the kidney and the mass of the liver, each of which contribute to the increased force necessary to manipulate the tissues. The remaining abdominal tissues tested fell within a range of $1.3 \mathrm{~N}$ to $2.5 \mathrm{~N}$.

Table 2.2: Abdominal probing force measurements

\begin{tabular}{cc}
\hline Tissue & Force $(\mathrm{N})$ \\
\hline Liver & 3.45 \\
Gallbladder & 1.32 \\
Spleen & 1.47 \\
Stomach & 2.53 \\
Kidney & 3.57 \\
Bladder & 1.53 \\
\hline
\end{tabular}

Experimental data collected to evaluate the force requirements for the suturing tasks of tissue puncture and knot tightening is shown in Table 2.3, where $n$ denotes 
the number of tests performed for each task. We can see that the tool force necessary for tissue puncture ranged from approximately 1.5 to $3.3 \mathrm{~N}$, while the force necessary for suture knot tightening ranged from 2.3 to $7.2 \mathrm{~N}$.

Table 2.3: Suture task force measurements

\begin{tabular}{lccc}
\hline Task & $\mathrm{n}$ & Force $(\mathrm{N})$ & $\mathrm{SD}$ \\
\hline Liver puncture & 1 & 3.26 & - \\
Gallbladder puncture & 3 & 1.49 & 0.72 \\
Size 4-0 knot tightening & 3 & 2.28 & 0.10 \\
Size 0 knot tightening & 2 & 7.19 & 0.96 \\
\hline
\end{tabular}

The forces necessary to achieve suture breakage are shown in Table 2.4, where the suture material tested was polypropylene (Surgilene, Davis \& Geck) unless otherwise noted. It is apparent that the force requirement to tighten the suture knot is dependent on the suture size.

Table 2.4: Suture break force measurements.

\begin{tabular}{cccc}
\hline U.S.P. Suture Size & $\mathrm{n}$ & Force $(\mathrm{N})$ & $\mathrm{SD}$ \\
\hline 1 (Polybutester) & 4 & 38.67 & 5.70 \\
3-0 & 6 & 11.93 & 0.15 \\
4-0 (Chromic Gut) & 2 & 8.73 & 1.71 \\
4-0 & 8 & 6.75 & 0.80 \\
5-0 & 2 & 4.84 & 0.51 \\
6-0 (Braided Polyester) & 1 & 5.10 & - \\
6-0 & 3 & 3.12 & 0.56 \\
7-0 & 2 & 2.20 & 0.34 \\
\hline
\end{tabular}

\subsubsection{Discussion}

The abdominal tissue manipulation results presented in Table 2.2 show that the greatest forces were measured for manipulation of the kidney and liver tissues. 
This result would be expected due to the rigidity of the kidney and the mass of the liver, each of which contribute to the increased force necessary to manipulate the tissues. Although not directly comparable, tissue handling in terms of grasping force have been presented in the literature to have a mean value of $8.5 \mathrm{~N}$ [103]. Thus, the forces recording during the abdominal organ manipulation experiment are expected to be within the safe range regarding the avoidance of tissue damage.

Suturing task forces, presented in Table 2.3, were found to range between 1.49 and $7.19 \mathrm{~N}$. The lowest force measured was for needle puncture of gallbladder tissue. However, a large standard deviation of $0.72 \mathrm{~N}$ over three trials was found and was likely caused by variable tissue stiffness within the organ. The greatest force was measured for suture knot tightening using sutures of size 0 . This result is expected due to the increase in bending stiffness of a suture line with a larger diameter. Finally, it was possible to compare the knot tightening force of size 4-0 suture with the literature. The results demonstrate that there is a significant dependence on the suture size when considering the force requirement to tighten the suture knot. This corresponds well with the work of Kitagawa et al. in which the average tension applied to the suture by an attending surgeon was slightly under $2 \mathrm{~N}$ for size 4-0 polypropylene [97]. Further, it was also shown that this force decreased with decreasing suture size. However, a definitive comparison between the two studies cannot be made due to dependence on the individual surgeon performing the operation. Lastly, the results for suture break force, seen in Table 2.4, show a range of 2.20 to $38.67 \mathrm{~N}$ with a direct correlation with suture size.

Based on these results, it is evident that there is a wide range of force requirements depending upon the task to be performed. Considering some examples of emerging surgical robots from the literature [35, 36, which were shown to have a load capacity of $5 \mathrm{~N}$, it can be seen that robots of this nature would be capable of performing many of the necessary tasks but would be limited to suture sizes of U.S.P. 5-0 or smaller. When comparing the results with the literature for size 4-0 polypropylene, it was found that the force of approximately $6.75 \mathrm{~N}$ presented here 
was much lower than the force of $13.76 \mathrm{~N}$ presented by Trail et al. [104]. One possible explanation for this result is the location of breakage. In the experiment conducted by Trail et al., the suture was secured by winding the line around two metal rods before taping it down. This would dictate that the suture failure must occur in the line. However for this study, the needle end of the suture was secured in a vice and it was observed that the majority of the failures occurred at the junction between the needle and the line. This would suggest that this junction is likely weaker than the tensile strength of the line. Since the focus of this study was to evaluate the surgical tool force requirements, and in particular for this experiment the force necessary to break the needle away from the rest of the suture, these results hold value. If it were determined that greater force was necessary to achieve a satisfactory knot, the surgeon would have the option of grasping the suture line in order to apply greater tension.

\subsubsection{Conclusions}

The results presented in Section 2.1.3 demonstrate that it is necessary for the designers of novel surgical robotics to consider the specific applications that their tool is targeting when determining the limits of capability. In a scenario such as this where increasing the force capacity of the tool may be size or cost prohibitive, it may be necessary to make use of specialized tools such as cutting instruments to achieve the forces that may be required for suturing tasks. The results presented here were developed under the assumption that the greatest forces required of a surgical robot would be found while performing tissue manipulation, suturing tasks, or suture needle separation. Under these conditions, the range of surgical tool forces was found to be $\in[1.32 N, 38.67 N]$. It is apparent that even for this relatively small sub-set of common surgical tasks, there is much variability in the necessary tool load capacities. Therefore, based on the results presented here and the work of others found in the literature, a load capacity of $5 \mathrm{~N}$ is suggested as 
a target goal as an end load on a manipulator for use in the design of endoscopic surgical tools used for abdominal procedures for which access is gained through a natural orifice as it provides a good trade-off between tool capability and the size of the tool.

\subsection{Workspace}

The workspace at the procedure location within the patient that the surgical manipulator must be able to maneuver around is highly dependent on the procedure being performed. Further, the skill level of the surgeon will have a significant effect on the amount of space required to perform the necessary manipulations for a given procedure. Data collected using a set of instrumented laparoscopic surgical tools shows that an expert surgeon can perform an example task within the confines of a cube of side length $5 \mathrm{~cm}$ where a novice may require a cube with a side length of 8 or $9 \mathrm{~cm}$ to perform the same task [105]. Similar requirements were found for a surgical instrument for surgery on the throat [58].

Another requirement previously mentioned is the necessity for the surgical instrument to achieve $180^{\circ}$ retroflexion. This requirement allows for the surgeon to access surgical sites in the upper abdomen when entering through the esophagus. However, the burden of meeting this requirement does not necessarily have to fall solely on the individual surgical manipulator, but instead on the endoscopic platform as a whole. This means that if retroflexion can be achieved by the endoscope that is delivering the manipulators to the surgical site, then the individual manipulators do not need to have the ability to retroflex. Assessment of common surgical practice has found that when using laparoscopic tools, the surgeon will spend $95 \%$ of the surgery within a cone with a vertex angle of $60^{\circ}$ [106. Thus, it is possible to trade bending range of motion for tool size or load capacity if necessary for a surgical manipulator design to be successful. 
Putting these two constraints together, a workspace with an edge length of $5 \mathrm{~cm}$ is a recommended target for the positional range of the manipulator spanning one end of the range limit to the other. Further, the manipulator should be capable of operating through an angular range of $60^{\circ}$ at a minimum for the majority of surgical manipulations. Under these conditions it is desirable for the manipulator to be capable of spanning the workspace within a $60^{\circ}$ bend/orientation range. However, under these conditions, a combination of manipulator bending and longitudinal positioning of the endoscope would need to be used to reach the proximal and distal limits of the workspace. Additional functionality in terms of the orientation range of the manipulator would reduce the necessity for longitudinal repositioning of the endoscope. The constraints suggested here preclude the use of excessively short length manipulators as well as manipulators with a small angular range of motion and long length.

\subsection{Safety Considerations}

The use of hydraulics in surgical applications faces some obstacles that need to be considered and overcome for this modality to be successful. One common theme in the field of hydraulics is that leakage of the hydraulic medium is difficult to prevent and must be expected. However, in the surgical application, there is little room for uncertainty when it comes to possible contamination of sensitive areas such as the peritoneal cavity [107]. This means that the hydraulic medium, such as saline, would need to be subject to the same sterility requirements as the rest of the device.

An additional concern stemming from the leakage of hydraulic fluid is any possible damage to nearby biological tissue due to the high operating pressures. Due to this high pressure, the hydraulic fluid would have the potential to escape the tool at high velocity and possibly cause damage by compression of sensitive tissues found 
within the abdominal cavity, as shown in Fig. 2.5. Indeed, the use of water jets have been proposed in the past as a means of tissue resection [108]. Therefore, it would be necessary to take this into consideration when designing the flow control in order to include safeguards against this sort of undesired behavior.

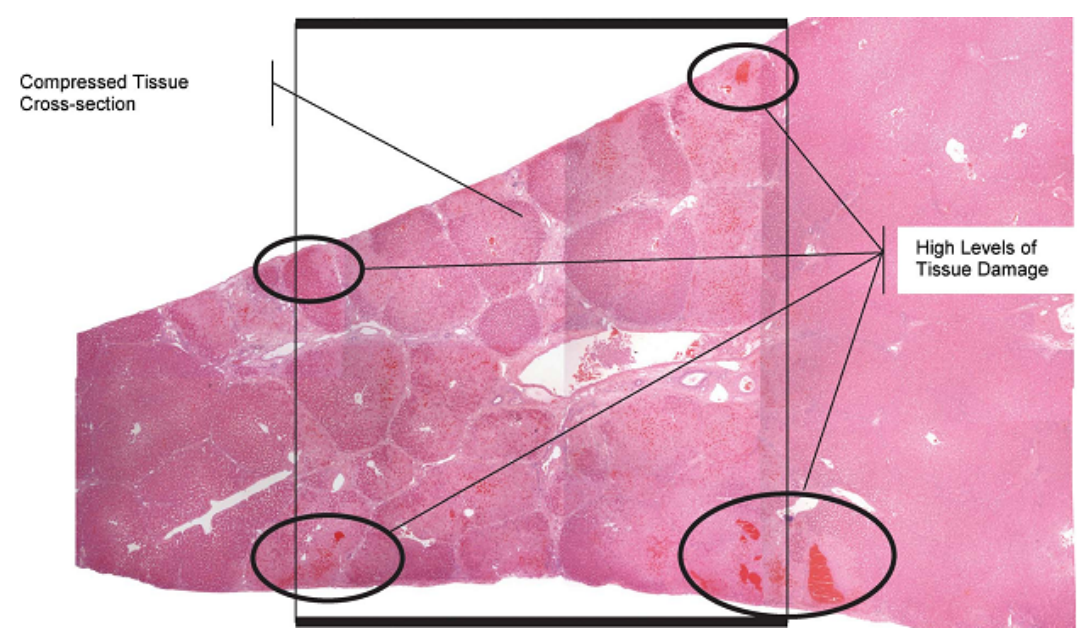

Figure 2.5: Example of tissue damage due to applied pressure [109].

The literature has shown that it can be difficult to predict whether or not a given load will result in damage to the tissue and further that the magnitude of the load that may cause damage varies significantly depending on what kind of tissue it is [109, 110, 111]. However, using these studies as general guidelines, it is possible to say that tissue damage begins to occur in the range of 160 to $280 \mathrm{kPa}$ of applied pressure [109, 110].

To find the pressure exerted on a tissue by a fluid leak it is assumed that the force applied to the tissue by the fluid results from a complete loss of forward momentum when the fluid impacts the tissue such that the force is calculated as

$$
F=m^{\prime} \Delta v
$$

where $m^{\prime}$ is the mass flow rate of the fluid and $\Delta v$ is the change in the forward velocity of the fluid. Then assuming that the velocity in the direction normal to 
the tissue surface is zero after impact, the equation can be written as

$$
F=\rho A v^{2}
$$

where $\rho$ is the fluid density, $A$ is the area of the hole, and $v$ is the velocity of the fluid as it exits through the hole. This relationship assumes that the fluid jet from the leak impacts the tissue normal to its surface and that the tissue is close enough to the site of the leak that the jet does not expand or lose velocity. If the velocity of the fluid is approximated using the orifice equation, the resulting pressure applied to the tissue at the point of impact is calculated as

$$
P=\frac{F}{A}=\rho v^{2}=2 C_{d}^{2} P_{i}
$$

where $C_{d}$ is the discharge coefficient of the leak orifice and $P_{i}$ is the pressure internal to the system at the leak site. Using an approximate discharge coefficient of 0.6 , this suggests that the pressure applied to the tissue could be as much as $72 \%$ of the supply pressure in the worst case scenario. It is also important, however, to consider the time over which the tissue is exposed to this pressure. Rodrigues et al. [111] showed that tissue subjected to sustained load from a suture began to rupture within approximately 15 to 20 seconds when the magnitude of the load was sufficiently high. Therefore, one possible method of reducing the risk of tissue damage would be to actively monitor for leakage and turn off the supply if leakage is detected, thus minimizing the amount of time that the tissue is exposed to unintended loads and lowering the risk of permanent tissue damage.

This safety concern could also be addressed through the introduction of a flow resistance within the supply line such that the downstream pressure drops significantly at high flow rates. For example, with the inclusion of an upstream flow restriction, the pressure drop across the restriction could be calculated as

$$
\Delta P=\frac{\rho}{2}\left(\frac{Q}{C_{d} A}\right)^{2}
$$


where the area of the flow orifice, $A$, would be sized such that the downstream pressure drops to a safer value when the flow rate, $Q$, through the orifice significantly exceeds the nominal flow rate of the system under normal conditions.

\subsection{Tool Size Constraints}

Another consideration for a robotic instrument to work in a surgical setting is that it must conform to the size constraints placed upon it by the anatomy of the patient. For natural orifice surgery, these constraints come primarily from the access pathways as they are significantly more restrictive than the available space within the abdominal cavity. In the case of transgastric access, the overall endoscope is constrained to a diameter in the range of 18 to $22 \mathrm{~mm}$ [52]. Within this cross-section must be contained vision channels (preferably stereoscopic), suction, irrigation, and at least two tool channels. An example layout of the endoscope cross-section is shown in Fig. 2.6. This layout permits the tool channels to have a diameter of approximately $6 \mathrm{~mm}$ as compared against a $20 \mathrm{~mm}$ endoscope. Within these tools channels, the components of the manipulator must be contained. For a two-segment manipulator, this would include two valves and six actuators. The actuators can be arranged serially as necessary to meet the overall size constraint. 


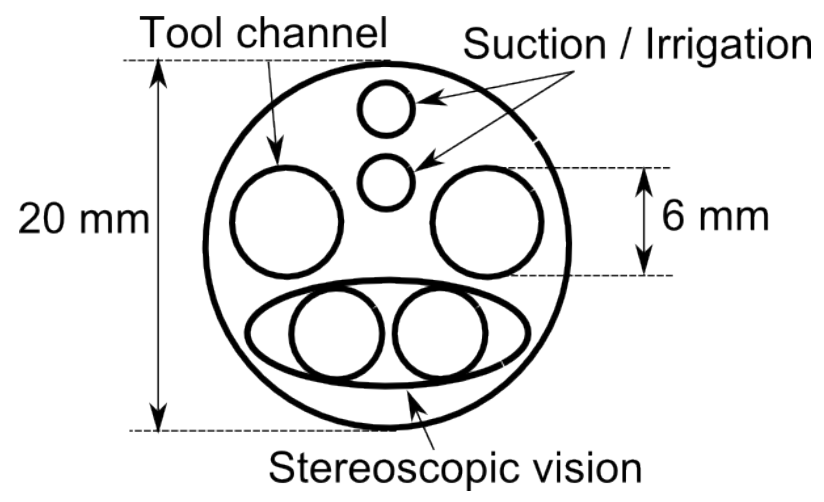

Figure 2.6: Example layout of endoscope cross-section with necessary channels. The manipulators described here would be contained within the tool channels.

\subsection{Summary of Design Requirements}

The considerations necessary for the design of a surgical manipulator are summarized here and separated into four primary areas. These considerations include the load capacity of the tool, the size of the workspace, safety of operation, and biological constraints on the overall size of the device.

First, considering the tool load requirements as laid out in Section 2.1 it is apparent that the tasks that a surgical tool may be expected to perform can vary widely. Further, these tasks and the associated load requirements are likely to differ greatly between the types of possible procedures. However, based on the conditions specific to abdominal procedures and taking into consideration the limitations of scale, it was suggested that a force load capacity of $5 \mathrm{~N}$ be the target load capacity for a surgical manipulator.

Next, the size of the workspace for the manipulator was examined in Section 2.2 through a review of the data from the literature collected for laparoscopic tools. From this information it was gathered that the tool workspace should be on the order of $50 \mathrm{~mm}$ in all three Cartesian directions to accommodate common surgical tasks such as suturing, cutting, or manipulation. Additionally, it was found that 
the majority of procedure manipulations take place within a cone rotated around the long axis of the tool with a vertex angle of $60^{\circ}$. This realization introduces some flexibility into the workspace requirements as it is now possible to transfer some of the manipulation range to the endoscope, which is used to deliver the manipulators to the surgical site, since it would not be unreasonable to re-position the endoscope between surgical tasks.

In considering the safe operation of a hydraulically powered manipulator within the abdominal cavity, there are several potential unique issues that must be included as discussed in Section 2.3. Of primary importance is the possibility of leakage which inherently necessitates the usage of a sterile hydraulic fluid such as standard saline. Additionally, in the event of leakage, it must be ensured that the leaking fluid will not result in unintended damage to the surrounding tissues. There are a few options for mitigating the risk of tissue damage. First, the flow rate of the fluid could be monitored and if a leak is indicated by a sharp unexpected increase in flow rate then the supply pump is switched off. Second, an upstream flow resistance could be used to induce a pressure drop if the flow rate should drastically increase due to a leak, thus reducing the risk of tissue due to exposure to high fluid pressure.

Finally, the tool size must be designed such that the instrument is biologically compatible with the patient and the intended access pathway. In the application to an esophageal access pathway, this limitation is on the order of $18-22 \mathrm{~mm}$ in diameter for the endoscope as a whole. Thus, considering the space available to the tool channels which would contain the surgical manipulators, the size limitation is likely to be constrained to $6 \mathrm{~mm}$ in diameter which must house all of the components necessary to operate the manipulator. 


\section{Chapter 3}

\section{Manipulator Backbone}

This chapter is the first of four that describe the design of the manipulator, each focusing on one particular aspect of the overall design. The focus of this chapter is the backbone (support) structure for the manipulator. First, Section 3.1 presents a discussion of the design choices including the selection of a continuum beam structure as the best fit for this design. In Sections 3.2 and 3.3 the methods of modeling a continuum beam are discussed to provide a reasonable simulation of the backbone component that will be revisited when considering the system assembly. Experimental results are then presented in Section 3.4 to provide a measure of the quality of the model under the prescribed assumptions.

\subsection{Motivation and Related Work}

There are a variety of technologies available to serve as the structure for a surgical robot. The most familiar robot form factor seen in industry is comprised of a small number of rigid links arranged in series. However, this style of robot is not suited for a natural orifice access technique as small motions can require large 
articulations and thus safety is obtained by providing the robot with room to move [47. The tight space requirements found in minimally invasive surgery discussed in Section 2.2 necessitate the use of a robotic structure with compliance and a high ratio of length to diameter. In this format, the primary options are hyperredundant robots with a high number of discrete links or robots with a continuum structure. Discrete link robots have the advantage of being more precise due to having a finite number of degrees of freedom, each of which can be actuated. However, for this style of robot to be feasible on a small scale, the geometry of the links and their connections must be relatively simple such as one degree of freedom pin joints. For a pin joint it would then be necessary to use two tendons/actuated inputs to control each degree of freedom thus increasing the robot complexity. Alternatively, continuum robots offer safe operation through natural compliance and ability to adapt to an unpredictable environment [47, 64]. This is due to the presence of an infinite number of degrees of freedom for the continuum robot while only a few of those degrees of freedom can be actuated. The downside to this is that the unactuated degrees of freedom passively determine the resulting beam shape under a given load which can make precise positioning difficult. As discussed in more detail in Section 6.2.2, for this application three actuators provide control over three degrees of freedom per manipulator segment. Thus in the example case of achieving six total degrees of freedom, six actuators could be used compared with twelve actuators for the example pin-jointed rigid link case. It is then the remaining, unactuated degrees of freedom that passively determine the beam shape. Further, the inherent compliance of the continuum robot makes it an attractive option for medical applications since the robot will conform to the unpredictable environment [112]. For this design, the continuum robot solution was selected to take advantage of its compliance and greater ease of miniaturization through simplicity.

The use of a continuous backbone for cable driven robots has been well documented in the literature [57, 67, 86, 87, 88, 113]. Continuum manipulators have 
many advantages that make them an ideal selection for this application including simplicity and dexterity [86]. By using a beam material with desirable high strain behavior such as nickel titanium alloys, it is possible to reach a wide range of positions as predicted using beam bending theory. This style of joint would include the flexible beam at the center with a variable number of disks attached to allow the tensile lines to be routed appropriately as shown in Fig. 3.1. This approach is similar to the hyper-redundant robots consisting of a multitude of rigid links, previously discussed, providing comparable yet distinct operating characteristics [113, 114].

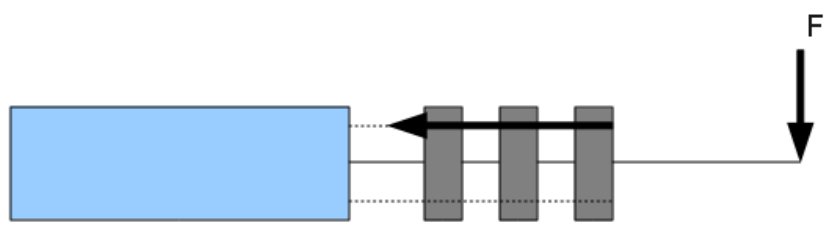

Figure 3.1: Graphic of beam bending based design.

Unfortunately, the prediction of performance for the continuous backbone manipulator is non-trivial and there have been numerous models developed to predict this behavior as discussed in Section 1.1.5 [55, 64, 67, 68]. However, many of them require significant computational effort and thus make it difficult to allow for practical application or they do not capture enough information to enable integration of the model into the model of a robotic system as a whole. Therefore, a discussion of the modeling of a continuous backbone for this application is presented here.

\subsection{Simplified Euler-Bernoulli Beam}

To enable modeling of beam behavior within the system, it is first necessary to decide on a method of describing the mechanics of the continuous beam. The task 
here is to allow for rapid prediction of manipulator behavior under the conditions of a cable driven continuum manipulator. If a model can be specified with reasonable accuracy, it can then be incorporated into a system model of the manipulator as a whole. One of the more common ways to capture the large-deflection behavior of the continuous backbone is to take the Euler-Bernoulli Theory and apply it to the special case of bending in a beam subjected to pure moment loading. The approximation of a tendon-driven robot as a beam subject to pure moment loading has been experimentally validated assuming proper selection of spacing and height of the tendon support disks [87, 115]. By neglecting arbitrary external loading, the solution to the Euler-Bernoulli Theory becomes trivial. While the assumptions of this model are overly constraining, this solution method is included here to provide insight into the predicted beam behavior under the strict conditions of pure moment load. Therefore, under the assumptions of uniform material properties, pure moment tendon driven loading, negligible shear deformation, and negligible external force loading, we start with the total potential energy function for the Euler-Bernoulli beam [116]

$$
\int_{0}^{L}\left[\frac{E I}{2} \theta^{\prime}(s)^{2}-m_{L}\left(\theta^{\prime}(s)-\theta_{o}\right)\right] d s
$$

where the first term represents the distortion energy in the beam and the remaining terms represent the external work of the applied moment load. Here it is assumed that the external loading is known and thus the energy contributed by the external load can be accounted for. Further, $\theta(s)$ represents the angle of the tangent to the curve of the beam at any location along its length measured relative to the undeformed configuration, $m_{L}$ is the moment being applied at the free end of the beam (see Fig. 3.2), and $E$ is the modulus of elasticity for the backbone material. In these equations and throughout the remainder of this dissertation except where otherwise noted, the prime denotes the first derivative of the variable with respect to the position along the beam, $s \in[0, L]$ where $L$ is the length of the beam. 
Now to minimize the total potential energy by minimizing the integrand, we choose $\theta(s)$ such that $\theta(0)=\theta_{o}$ and $\theta^{\prime}(s)=\mathcal{K}$ minimizes $\frac{E I}{2} \theta^{\prime 2}-m_{L} \theta^{\prime}$. This yields $\mathcal{K}=$ $\frac{m_{L}}{E I}$, which then provides the unique minimizer $\theta(s)$ that satisfies the boundary conditions as

$$
\theta(s)=\frac{m_{L}}{E I} s+\theta_{o}
$$

Using this solution, the resulting shape of the beam can be expressed as a circle with a radius of

$$
r_{\text {beam }}=\frac{E I}{m_{L}}
$$

as depicted in Fig. 3.2, which is consistent with the basic constitutive relationship for the Euler-Bernoulli beam [117, 118].

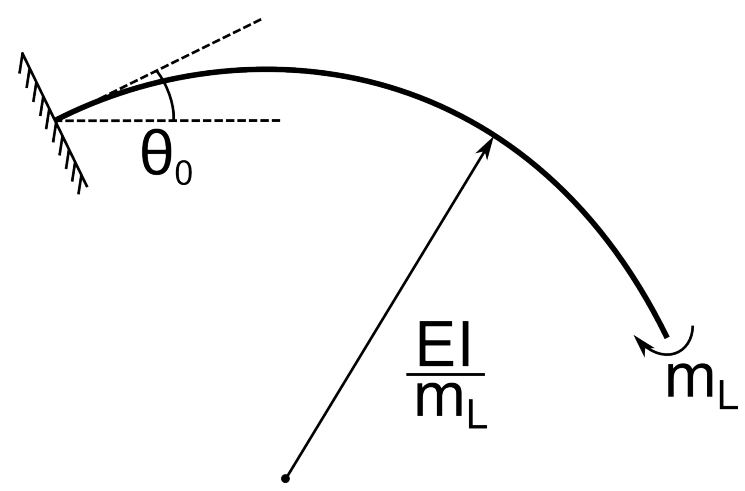

Figure 3.2: Resulting beam geometry from an end applied moment.

The resulting radius of curvature as calculated in Eq. 3.3 can be used to find the tool tip location by expressing the coordinates of this point in both the longitudinal (z) and transverse (x) axes, as shown in Fig. 3.3. These equations are found to be,

$$
\begin{gathered}
z_{\text {end }}=r_{\text {beam }} \sin \left(\frac{l}{r_{\text {beam }}}\right) \\
x_{\text {end }}=r_{\text {beam }}\left[1-\cos \left(\frac{l}{r_{\text {beam }}}\right)\right],
\end{gathered}
$$

where $l$ is the length of the beam segment. This result suggests that it is possible to achieve a smooth motion of the tool tip when using a structure based on beam 
bending principles and a controlling force applied as a moment. This result has been confirmed in the literature where it has been shown experimentally that a cantilever beam subject to moment loading assumes the shape of a circular arc in the absence of external loading [87].

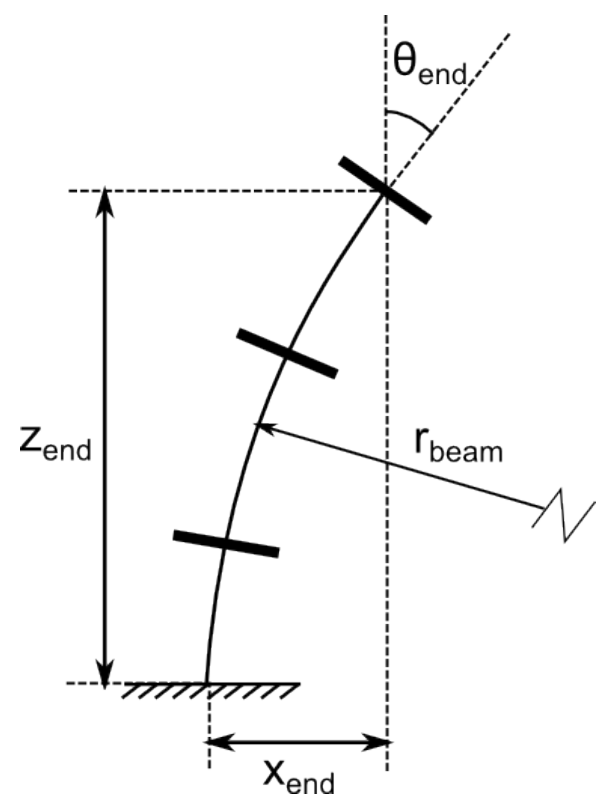

Figure 3.3: Graphic showing location of the beam tip.

The limitation of this forward kinematic model is that it only accounts for moment loading at the tip. Any forces or other external loading applied cannot be accounted for in the same simple manner. For this reason it is necessary to approach the problem from a different direction that allows for greater flexibility in terms of loading scenarios and more realistic usage cases. 


\subsection{Model Prediction of Beam Shape and End Loads}

An alternative approach to predicting the behavior of a continuous backbone will be presented in this section. This model makes use of the principles of inverse kinematics and potential energy minimization to predict beam shape and end load conditions for a specified end position and orientation of a given beam segment in a manner similar to the classical Cosserat rod model [115. The model begins by assuming that the backbone of each manipulator segment is modeled as an EulerBernoulli beam which is cantilevered at the base, similar to what was previously discussed. However, in this case the externally applied loading is not known and thus is not factored in to the total energy. Assuming that the position and orientation of the distal end is given, we wish to determine the beam shape and the beam reaction force and moment acting on the end-effector at the distal end of the beam.

The method of modeling the deformation of a robot with a continuous backbone which will be presented here is an energy-based formulation [119]. The basis of this method is that of all the beam shapes that satisfy the specified end point conditions, the continuous backbone with negligible mass will assume the shape with the least stored elastic deformation energy. Knowing this shape for the specified location and orientation of the distal end of the beam, it is then possible to calculate the resulting load applied on the terminating disk located at the distal end of the beam which can include a force vector and a moment vector of arbitrary directions. This inverse kinematics, quasi-static model, which neglects the effects of the mass of the beam itself [86, 88, is here referred to as the Inverse Formulation Model (IFM). 


\subsubsection{IFM in Two-Dimensions}

In the two-dimensional case, the problem simplifies to a cantilevered beam with arbitrary position $(z$ and $x$ ) and orientation $(\theta)$ end conditions as shown in Fig. 3.4. Under these end conditions, the beam will have some shape which, if known, provides additional information about what conditions (load and/or moment) are present at the distal end.

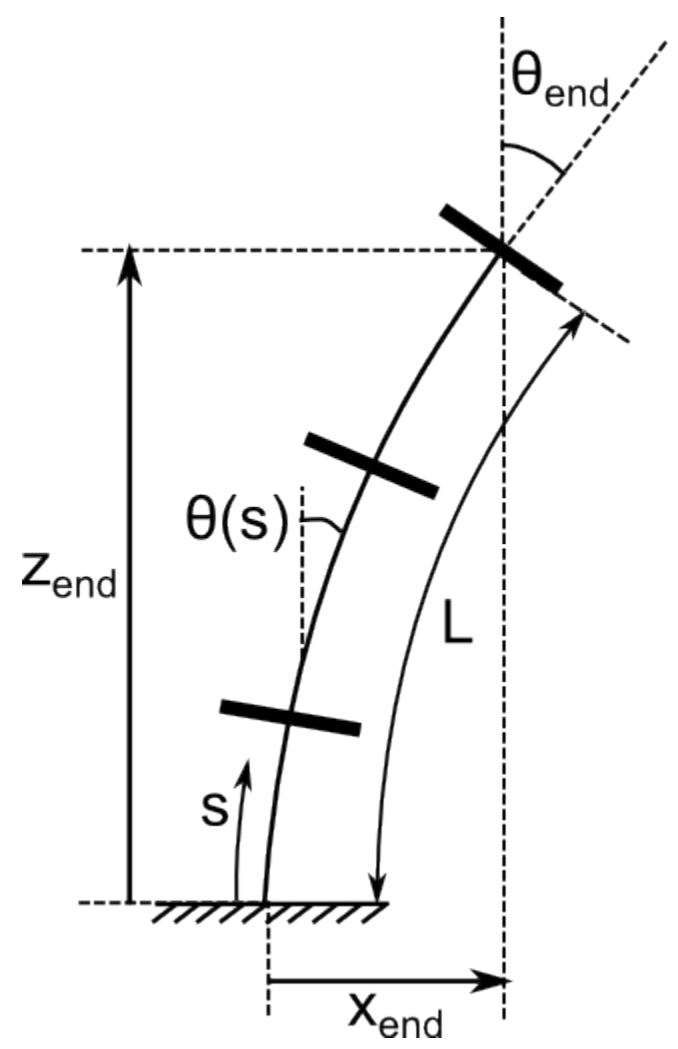

Figure 3.4: Graphic showing the conditions of a cantilevered beam with arbitrary end coordinates.

The IFM in two-dimensions assumes planar bending only and thus can only be used to describe in-plane external loading. Let $s \in[0, L]$ denote the distance along the beam of length $L$, and $\theta(s)$ be the angular orientation at location $s$. Thus, $\theta(s)$ is used to describe the shape of the beam for a given set of position 
and orientation coordinates for the distal end. In other words, the beam shape is specified as a n-degree parameterization of $\theta(\cdot)$ with respect to $s$, assuming that the location and orientation of the distal end of the beam are specified as inputs such that $x_{e n d}, z_{\text {end }}$, and $\theta_{\text {end }}$ are known. The parameterization of $\theta(\cdot)$ can take many forms; however, the use of a polynomial function and a spline function are evaluated here. For a selected parameterization method, the beam shape is computed using the potential energy stored within the beam.

Potential energy is stored within the beam of cross-sectional area $A$, elastic modulus $E$, second moment of area $I$, and shear modulus $G$, as elastic deformation consisting of axial, bending, and shear components with stiffness terms of $E A$, $E I$, and $G A$, respectively [119]. This formulation differs from what was presented in Eq. 3.1 in that it includes terms for axial and shear strain and does not include terms for external work since the external loading is not known a priori and thus only quantifies stored energy. Using these definitions, the potential energy within the beam can be expressed as

$$
P E(\theta(\cdot))=\frac{1}{2} \int_{0}^{L}\left[\frac{1}{2} \vec{v}(s)^{T} Q(s) C Q(s)^{T} \vec{v}(s)+E I \theta^{\prime}(s)^{2}\right] d s
$$

where the prime denotes the first derivative of the variable with respect to the position along the beam, $s$ as before and $Q(s), C, \vec{v}(s)$, and $\vec{x}(s)$ are defined as

$$
\begin{aligned}
& Q(s)=\left[\begin{array}{cc}
\cos (\theta(s)) & -\sin (\theta(s)) \\
\sin (\theta(s)) & \cos (\theta(s))
\end{array}\right] \\
& C=\left[\begin{array}{cc}
E A & 0 \\
0 & G A
\end{array}\right] \\
& \vec{v}(s)=\left[\begin{array}{c}
z(s)^{\prime}-\cos (\theta(s)) \\
x(s)^{\prime}-\sin (\theta(s))
\end{array}\right] \\
& \vec{x}(s)=\left[\begin{array}{l}
z(s) \\
x(s)
\end{array}\right]
\end{aligned}
$$


By these definitions, $Q(s)$ represents the cross-sectional orientation as a function of $s, C$ is used to collect the axial and shear stiffness terms, and $\vec{v}(s)$ measures axial and shear stretch of the beam. The vector $\vec{x}(s)$ represents the position vector of the beam center line as a function of $s$.

Using this definition of the potential energy, the beam shape is predicted using a constrained optimization to minimize the potential energy stored within the beam subject to the boundary conditions at $s=L$,

$$
x(L)=x_{\text {end }}, \quad z(L)=z_{\text {end }}, \quad \theta(L)=\theta_{\text {end }}
$$

and at $s=0$, the cantilevered beam boundary conditions

$$
x(0)=0, \quad z(0)=0, \quad \theta(0)=0 .
$$

Evaluating the energy function through the application of Hamilton's principle [120] as discussed in the literature results in the following partial differential equations governing the beam, adjusted for the static case [119]

$$
\begin{array}{r}
{\left[Q(s) C Q^{T}(s) \vec{v}(s)\right]^{\prime}=\overrightarrow{0}} \\
E I \theta^{\prime \prime}(s)+\vec{x}^{\prime T}(s) S Q(s) C Q^{T}(s) \vec{v}(s)=0
\end{array}
$$

where $S$ is the skew-symmetric matrix

$$
S=\left[\begin{array}{cc}
0 & 1 \\
-1 & 0
\end{array}\right]
$$


These differential equations are further subject to the following boundary conditions.

$$
\begin{aligned}
E I \theta^{\prime}(L) & =M_{b} \\
\left.Q C Q^{T} \vec{v}\right|_{L} & =\overrightarrow{F_{b}} \\
\theta(0) & =0 \\
\vec{x}(0) & =0 .
\end{aligned}
$$

These boundary conditions designate that the proximal end of the beam is located at the origin, is clamped at $s=0$, and that the end loads are defined as $\overrightarrow{F_{b}}$ and $M_{b}$ with respect to the global coordinate system at $s=L$.

Examination of this model reveals that the majority of the deformation of the beam will result from bending deformations due to lower energy requirements. For example, for the beam used experimentally in Section 3.4, the stiffness quantities are $E I=5.6361 \cdot 10^{-5} \mathrm{~N} \cdot m^{2}, E A=4453.2 \mathrm{~N}$, and $G A=1590.4 \mathrm{~N}$. Therefore, it is possible to save significant computational effort by reducing the potential energy function to the classical model form which accounts for only energy from bending deformations as

$$
P E(\theta(\cdot))=\frac{1}{2} \int_{0}^{L} E I \theta^{\prime}(s)^{2} d s
$$

A justification of this decision is presented in Section 3.4. With a given solution based on the minimization of the strain energy, the beam shape can be specified using the positional coordinates of the centerline, $x(s)$ and $z(s)$, which, since the relative position of the end point of a differential element of the beam at $s$ is $[\sin (\theta(s)) ; \cos (\theta(s))] d s$, can be given as functions of $\theta(s)$ such that

$$
\begin{array}{ll}
x(s)=\int_{0}^{s} \sin (\theta(\sigma)) d \sigma, & s \in[0, L] \\
z(s)=\int_{0}^{s} \cos (\theta(\sigma)) d \sigma, & s \in[0, L]
\end{array}
$$


An optimization algorithm is used to minimize the stored strain energy within the beam in order to find the lowest energy beam shape corresponding to a set of end conditions. This is accomplished by parameterizing $\theta(s)$ as a high order polynomial and using a constrained optimization to minimize the potential energy (Eq. 3.20 , subject to the constraints of Eqs. 3.11 and 3.12 , within the beam as calculated above over all possible combinations of the parameterization coefficients. An example of the output from this model is shown in Fig. 3.5 for which the bending stiffness, EI, was $5.6361 \cdot 10^{-5} \mathrm{~N} \cdot \mathrm{m}^{2}$. Noting, however, that the beam shape prediction is independent of $E I$ assuming that both are constant along the beam. Inputs to the model were $30 \mathrm{~mm}$ for both the transverse and longitudinal tip positions and an angle of $10^{\circ}$ for the tip orientation.

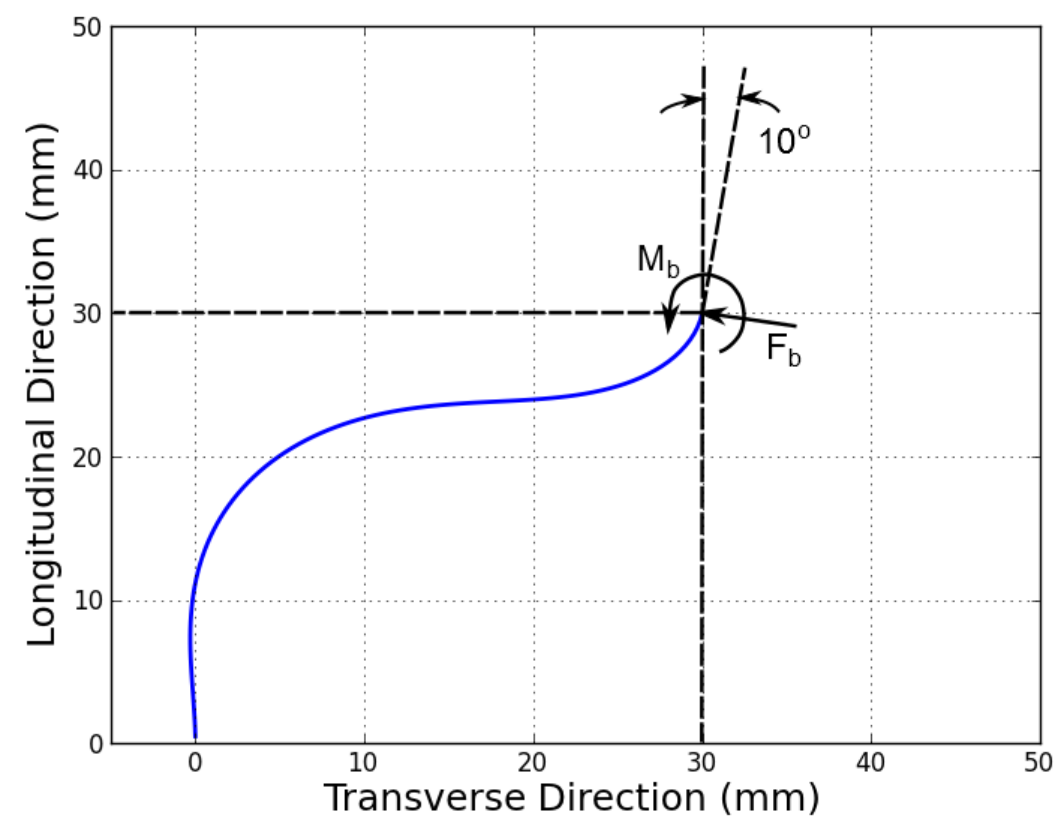

Figure 3.5: Example backbone model output for arbitrary input parameters.

If boundary conditions corresponding to the constant curvature load case are applied, it is found that the 2D IFM output using a 6th order polynomial parameterization for $\theta(s)$ matches the beam shape predicted by the basic constant curvature model. This is demonstrated in Fig. 3.6, where the beam shape from 
each model is plotted for a variety of constant curvature input conditions with $\theta(L) \in\left[15^{\circ}, 150^{\circ}\right]$. As can be seen, the curves from each model perfectly overlap for all inputs. Further, comparing the model predicted end loads from the IFM with that of the constant curvature model it was found that they compare well. Moment loads matched the loads specified by Eq. 3.3 and force loads were zero, noting however that small deviations in position and orientation occasionally result in large changes in the predicted end load.

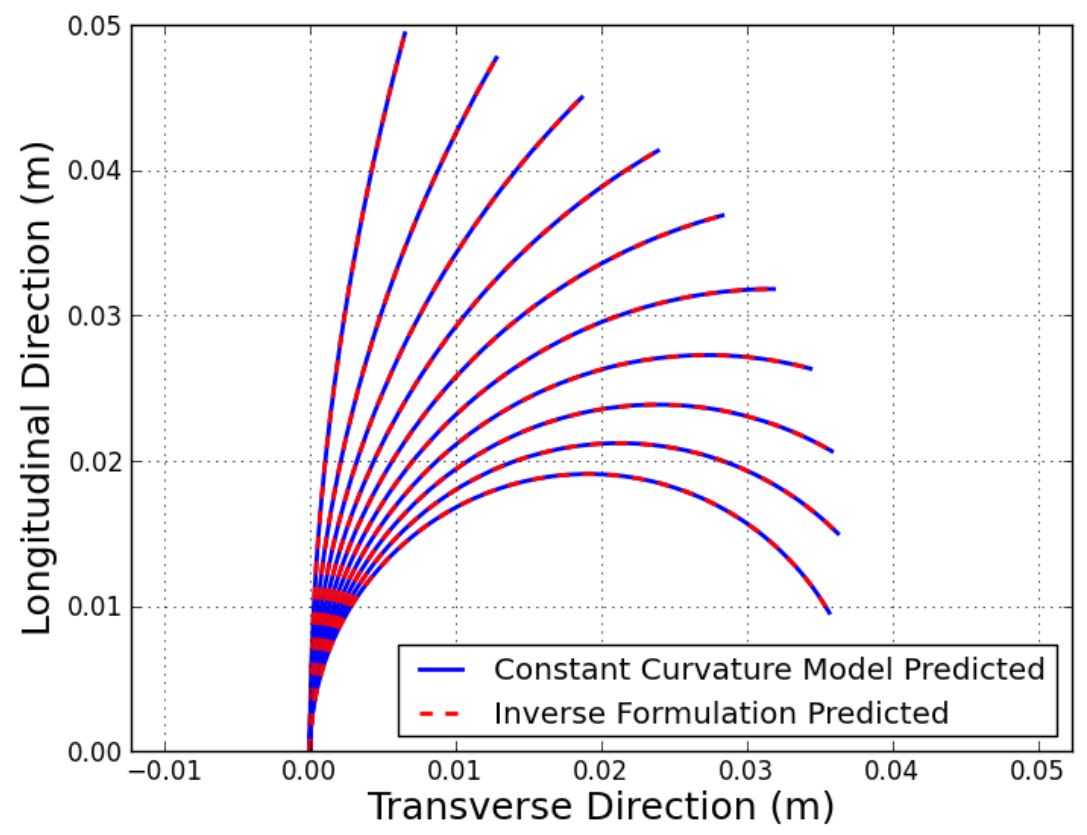

Figure 3.6: Comparison of IFM output with that of constant curvature model for constant curvature input conditions.

Once the potential energy of the beam has been minimized using the optimization algorithm, the beam shape is known and the end loads can be calculated. This is done using the principle of virtual work which states that the load corresponding to a given displacement can be computed if the associated potential energy is known [118]. Under this principle, the three relevant end loads, $\left(F_{b, x}, F_{b, z}, M_{b}\right)$, 
can be calculated as

$$
F_{b, x}=\frac{\partial P E^{*}}{\partial x_{\text {end }}}, \quad F_{b, z}=\frac{\partial P E^{*}}{\partial z_{\text {end }}}, \quad M_{b}=\frac{\partial P E^{*}}{\partial \theta_{\text {end }}}
$$

where $P E^{*}$ is the potential energy stored within the beam as calculated by the optimization. These values can be computed directly using the Lagrange multipliers associated with each of the end position and orientation constraints.

\subsubsection{IFM in Three-Dimensions}

Similar to the two-dimensional model, the IFM in three-dimensions is based on the description of the beam as a smooth parameterized curve. However, for the threedimensional model, the beam shape must be described by three successive rotation angles, $\phi(s), \theta(s)$, and $\psi(s)$, which are Euler angles representing the orientation of the beam cross-section with respect to the $x, y^{\prime}$, and $z^{\prime \prime}$ axes (the rotated local coordinate axes), respectively, as shown in Fig. 3.7. By this definition, the reference configuration of the beam originates at the origin and is tangent to the Z-axis of the reference coordinate system.

The rotation matrix, $R(s) \in S O(3)$, used to represent this series of rotations about the local coordinate system contains the intrinsic Euler rotations about the $x$-axis by $\phi(s)$, then about the $y^{\prime}$-axis (new y-axis) by $\theta(s)$, and finally about the $z^{\prime \prime}$-axis (new z-axis) by $\psi(s)$ (see Fig. 3.7) and is realized as shown in Eq. 3.24. The prime notation on the axis labels indicates that the axis has changed orientation as a result of previous rotations. 
$R(s)=R_{\phi} R_{\theta} R_{\psi}=$

$\left[\begin{array}{ccc}\cos \theta \cos \psi & -\cos \theta \sin \psi & \sin \theta \\ \cos \phi \sin \psi+\sin \phi \sin \theta \cos \psi & \cos \phi \cos \psi-\sin \phi \sin \theta \sin \psi & -\sin \phi \cos \theta \\ \sin \phi \sin \psi-\cos \phi \sin \theta \cos \psi & \sin \phi \cos \psi+\cos \phi \sin \theta \sin \psi & \cos \phi \cos \theta\end{array}\right]$

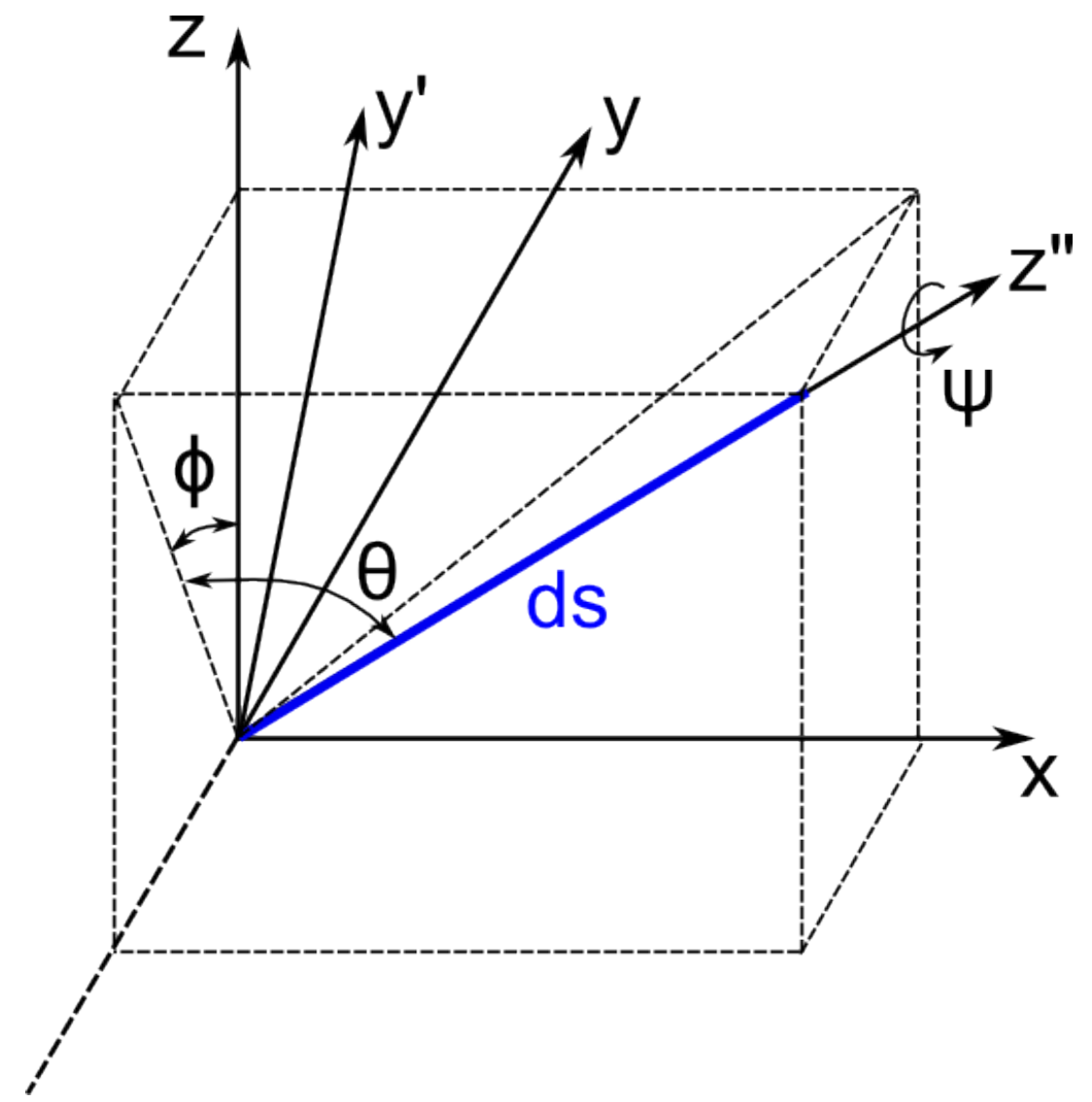

Figure 3.7: Three dimensional Cartesian coordinate system showing orientation angles for a differential beam segment, $d s$. The $y^{\prime}$-axis represents the new, rotated $\mathrm{y}$-axis and the $z^{\prime \prime}$-axis represents the new rotated z-axis.

Using the rotation matrix, it is possible to find the skew symmetric matrix which 
contains the two bending components as well as the torsion component that together describe the beam shape and orientation as

$$
u(s)=R(s)^{T} R(s)^{\prime}=\left[\begin{array}{ccc}
0 & -u_{z} & u_{y} \\
u_{z} & 0 & -u_{x} \\
-u_{y} & u_{x} & 0
\end{array}\right]
$$

where $u_{x}$ and $u_{y}$ relate to bending about the local $x$ and $y$ axes, respectively, and $u_{z}$ relates to torsion about the local z-axis [115]. Evaluating this expression and pulling out the functions for $u_{x}, u_{y}$, and $u_{z}$ results in

$$
\vec{u}(s)=\left[\begin{array}{l}
u_{x} \\
u_{y} \\
u_{z}
\end{array}\right]=\left[\begin{array}{c}
\sin \psi(s) \theta(s)^{\prime}+\cos \psi(s) \cos \theta(s) \phi(s)^{\prime} \\
\cos \psi(s) \theta(s)^{\prime}-\cos \theta(s) \sin \psi(s) \phi(s)^{\prime} \\
\sin \theta(s) \phi(s)^{\prime}+\psi(s)^{\prime}
\end{array}\right]
$$

Similarly, the differential expression of $x(s), y(s)$, and $z(s)$, which define the coordinates of the beam centerline, can be specified as

$$
\frac{d \vec{x}(s)}{d s}=\left[\begin{array}{c}
x^{\prime}(s) \\
y^{\prime}(s) \\
z^{\prime}(s)
\end{array}\right]=R(s)\left[\begin{array}{l}
0 \\
0 \\
1
\end{array}\right]
$$

Further, the resultant beam curvature, $\mathcal{K}(s)$, is calculated as the magnitude of the arc length derivative of the tangent vector where the tangent vector of the curve at any location is found as $\vec{T}(s)=\vec{x}(s)^{\prime}$ [121, 122]. Evaluating these expressions for the rotation matrix from Eq. 3.24 yields the following expression for beam curvature

$$
\mathcal{K}(s)=\sqrt{u_{x}^{2}+u_{y}^{2}}=\sqrt{\theta^{\prime}(s)^{2}+\phi^{\prime}(s)^{2} \cos ^{2} \theta(s)}
$$

with the values $\theta^{\prime}(s)$ and $\phi^{\prime}(s)$ defined as the differential change in $\theta(s)$ and $\phi(s)$ over the segment, respectively. Similarly, the torsion, $\tau(s)$, comes directly from 
$u_{z}$ such that

$$
\tau(s)=\sin \theta(s) \phi(s)^{\prime}+\psi(s)^{\prime}
$$

Thus, for the deformed beam which traces some curved path defined by the above parameterization, the potential energy stored within the beam can now be expressed as a function of the beam curvature and torsion as

$$
P E(\phi(\cdot), \theta(\cdot), \psi(\cdot))=\frac{1}{2} \int_{0}^{L}\left[E I \mathcal{K}(s)^{2}+G J \tau(s)^{2}\right] d s
$$

where $E I$ is the bending stiffness and $G J$ is the torsional stiffness noting that $J=2 I$ for a differential segment of a circular cross-section. This function for the potential energy is of the same form as was shown in Eq. 3.20 with the addition of torsional energy, but still neglecting axial extension and direct shear which are typically negligible [115]. This formulation of potential energy can be equivalently represented using the components of the skew symmetric matrix directly, $\vec{u}(s)=\left[u_{x}, u_{y}, u_{z}\right]^{T}$, when the expression $\vec{u}(s)^{T} \cdot K_{B T} \cdot \vec{u}(s)$ is substituted for the integrand in Eq. 3.30. Here $K_{B T}$ is the stiffness matrix represented as

$$
K_{B T}=\left[\begin{array}{ccc}
E I & 0 & 0 \\
0 & E I & 0 \\
0 & 0 & G J
\end{array}\right]
$$

Again, assuming that the location and orientation of the distal end of the beam are specified as inputs such that $x_{e n d}, y_{\text {end }}, z_{\text {end }}, \phi_{\text {end }}, \theta_{\text {end }}$, and $\psi_{\text {end }}$ are known, and integrating $\vec{x}(s)^{\prime}$ to get the vector components defining the beam centerline, 
$x(s), y(s)$, and $z(s)$ as

$$
\begin{array}{ll}
x(s)=\int_{0}^{s} \sin (\theta(\sigma)) d \sigma, & s \in[0, L] \\
y(s)=\int_{0}^{s}-\cos (\theta(\sigma)) \sin (\phi(\sigma)) d \sigma, & s \in[0, L] \\
z(s)=\int_{0}^{s} \cos (\theta(\sigma)) \cos (\phi(\sigma)) d \sigma, & s \in[0, L],
\end{array}
$$

the three-dimensional beam shape is then given by the constrained optimization of the potential energy of the beam subject to boundary conditions at $s=L$,

$$
\begin{aligned}
& x(L)=x_{\text {end }}, \quad y(L)=y_{\text {end }}, \quad z(L)=z_{\text {end }}, \\
& \phi(L)=\phi_{\text {end }}, \quad \theta(L)=\theta_{\text {end }}, \quad \psi(L)=\psi_{\text {end }}
\end{aligned}
$$

and at $s=0$

$$
\vec{x}(0)=0, \quad \phi(0)=0, \quad \theta(0)=0, \quad \psi(0)=0
$$

As in the two-dimensional case, once the potential energy of the beam has been minimized, the beam shape is known and the end load can be calculated using the principle of virtual work, realized as

$$
\begin{gathered}
F_{b, x}=\frac{\partial P E^{*}}{\partial x_{\text {end }}}, \quad F_{b, y}=\frac{\partial P E^{*}}{\partial y_{\text {end }}}, \quad F_{b, z}=\frac{\partial P E^{*}}{\partial z_{\text {end }}}, \\
M_{b, \phi}(L)=\frac{\partial P E^{*}}{\partial \phi_{\text {end }}}, \quad M_{b, \theta}(L)=\frac{\partial P E^{*}}{\partial \theta_{\text {end }}}, \quad M_{b, \psi}(L)=\frac{\partial P E^{*}}{\partial \psi_{\text {end }}}
\end{gathered}
$$

where again $P E^{*}$ is the potential energy stored within the beam as calculated by the optimization. The moments calculated in Eq. 3.39 are with respect to the local rotated coordinate axes. 
An example of the output from this model is shown in Fig. 3.8 where

$$
\left[\begin{array}{l}
x_{\text {end }} \\
y_{\text {end }} \\
z_{\text {end }} \\
\phi_{\text {end }} \\
\theta_{\text {end }} \\
\psi_{\text {end }}
\end{array}\right]=\left[\begin{array}{c}
20 \mathrm{~mm} \\
10 \mathrm{~mm} \\
35 \mathrm{~mm} \\
5^{\circ} \\
10^{\circ} \\
15^{\circ}
\end{array}\right]
$$

For this example, the orientation angles were parameterized using 6th order polynomials as in Section 3.3.1 and the material and geometric properties of the beam were $E=28.0 \mathrm{GPa}, G=10.8 \mathrm{GPa}, L=50 \mathrm{~mm}, I=0.002 \mathrm{~mm}^{4}$, and $J=0.004 \mathrm{~mm}^{4}$. Then using Eqs. 3.38 and 3.39, the calculated end loads are

$$
\left[\begin{array}{c}
F_{x} \\
F_{y} \\
F_{z} \\
M_{\phi} \\
M_{\theta} \\
M_{\psi}
\end{array}\right]=\left[\begin{array}{c}
0.43 N \\
0.22 N \\
0.91 N \\
0.0016 N \cdot m \\
0.0061 N \cdot m \\
-0.0007 N \cdot m
\end{array}\right]
$$




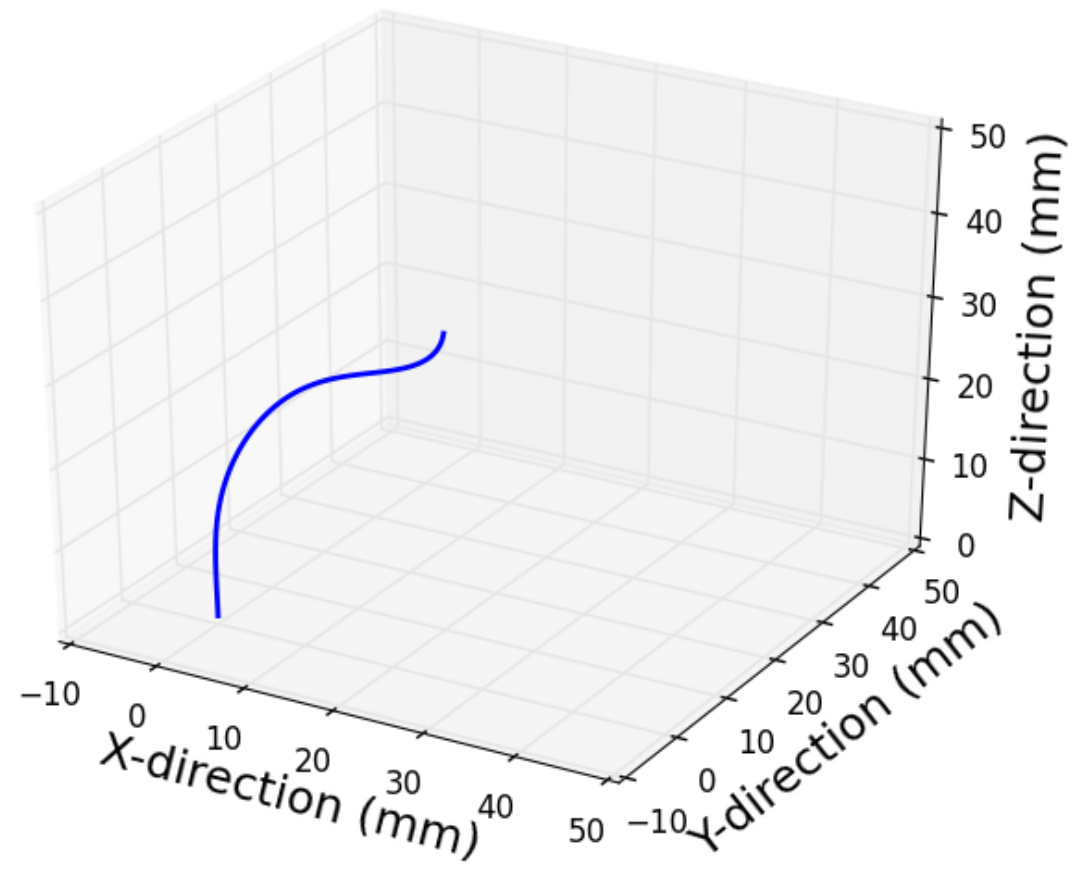

Figure 3.8: Example of three-dimensional IFM output for arbitrary input parameters.

\subsection{Experimental Model Validation}

To test the effectiveness of the inverse formulation model at predicting the beam shape for a given loading, an experimental setup was prepared consisting of a flexible nickel titanium alloy beam $(E=28.0 G P a, G=10.8 G P a)$ [123], support structure, and a six-axis load cell (Nano25, ATI Industrial Automation). The length of the beam was $50 \mathrm{~mm}$ for all test conditions with the exception of SubFig. reference (b) (Table 3.1), which had a length of $55 \mathrm{~mm}$, and a circular cross section with a diameter of $0.45 \mathrm{~mm}$. Position and orientation of the distal end of the beam as well as the beam shape was recorded photographically (see Fig. 3.9 and a grid backdrop was used to provide scale. A variety of combinations of position and orientation were selected randomly, the values for which are shown in Table 3.1. For each combination, the beam was allowed to assume a stable 
configuration and the beam was not observed to pass through a bifurcation into an unstable configuration.

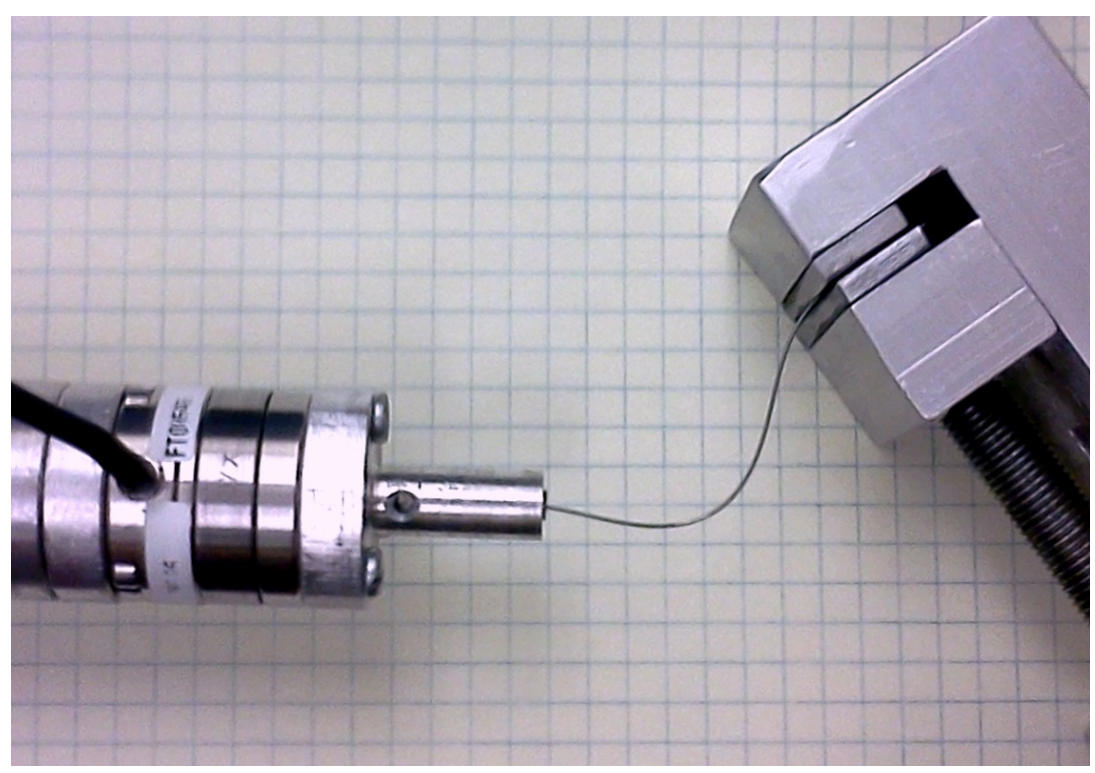

Figure 3.9: Example photograph of experimental setup used to record beam shape for arbitrary position and orientation of distal end.

Table 3.1: Distal end location and orientation conditions arranged according to corresponding sub-figure references.

\begin{tabular}{cccc}
\hline $\begin{array}{c}\text { Sub- } \\
\text { Fig. }\end{array}$ & $\begin{array}{c}z_{\text {end }} \\
(\mathrm{mm})\end{array}$ & $\begin{array}{c}x_{\text {end }} \\
(\mathrm{mm})\end{array}$ & $\begin{array}{c}\theta_{\text {end }} \\
\left({ }^{\circ}\right)\end{array}$ \\
\hline (a) & $39.7 \pm 0.7$ & $27.3 \pm 0.7$ & $0 \pm 2$ \\
(b) & $28.0 \pm 1.0$ & $35.0 \pm 1.0$ & $115 \pm 2$ \\
(c) & $33.0 \pm 0.4$ & $25.0 \pm 0.4$ & $45 \pm 2$ \\
(d) & $40.1 \pm 0.4$ & $27.0 \pm 0.4$ & $60 \pm 2$ \\
(e) & $34.9 \pm 0.5$ & $30.0 \pm 0.5$ & $-2.5 \pm 2$ \\
(f) & $46.6 \pm 0.4$ & $7.4 \pm 0.4$ & $-60 \pm 2$ \\
\hline
\end{tabular}

For each combination of position and orientation, the beam shape was recorded using image pixels to measure beam location and transferred to a data file. Next, 
the same set of position and orientation conditions were used in the inverse formulation model described in Section 3.3 .

Table 3.2: Model predicted end loads using both the full potential energy function (Eq. 3.6) and the simplified energy function (Eq. 3.20). Prediction of stored potential energy, $P E^{*}$, also included for comparison. Forces are given in N, moments and potential energy in $N \cdot m$.

\begin{tabular}{c|cccc|cccc}
\hline Sub- & \multicolumn{3}{|c|}{ Simplified PE Function } & \multicolumn{4}{c}{ Full PE Function } \\
Fig. & $F_{z}$ & $F_{x}$ & $M_{y}$ & $P E^{*}$ & $F_{z}$ & $F_{x}$ & $M_{y}$ & $P E^{*}$ \\
\hline (a) & 0.13 & -0.11 & 0.0039 & 0.0025 & 0.14 & 0.13 & -0.0021 & 0.0022 \\
(b) & 0.02 & 0.07 & -0.0020 & 0.0022 & 0.02 & 0.05 & -0.0017 & 0.0019 \\
(c) & 0.57 & 0.38 & 0.0054 & 0.0050 & 0.51 & 0.36 & 0.0048 & 0.0046 \\
(d) & 0.01 & -0.02 & -0.0007 & 0.0006 & 0.01 & -0.02 & -0.0010 & 0.0006 \\
(e) & 0.27 & -0.03 & 0.0045 & 0.0037 & 0.25 & -0.03 & 0.0051 & 0.0034 \\
(f) & 0.18 & -0.24 & 0.0066 & 0.0036 & 0.20 & -0.31 & 0.0084 & 0.0032 \\
\hline
\end{tabular}

First, comparing the predictions made using the planar 2D models for the simplified energy function (Eq. 3.20) against the full energy function (Eq. 3.6), the model predicted beam shapes for each of the end point positions and orientations (specified in Table 3.1) showed no change based upon which energy function was used (see Fig. 3.10). This suggests that the inclusion of the strain energy from direct shear is negligible for this application. Further, making this simplification reduced the average computational time for these six test cases from 1.71 seconds to 0.15 seconds.

A comparison of the predicted end loads and stored potential energy between the IFM using the full potential energy function (Eq. 3.6) and the simplified energy function (Eq. 3.20) is shown in Table 3.2. The results show some variability between each predicted end load. This is expected due to the differences in the formulations. However, the discrepancy is often not large and thus justifies the simplification of optimizing only bending energy in the calculation in order to realize the gains in computational efficiency. 


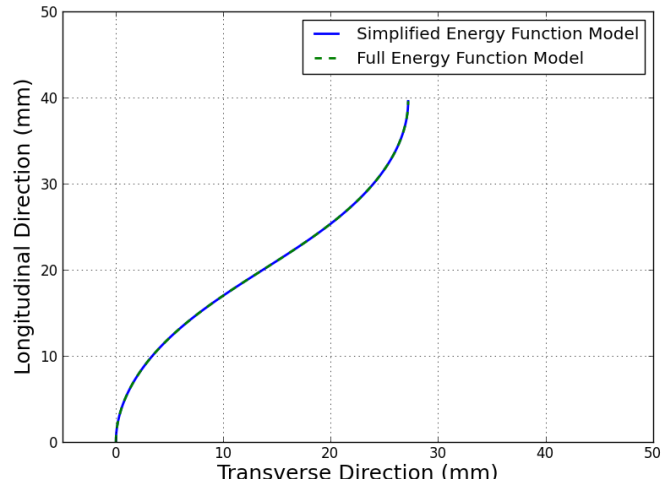

(a)

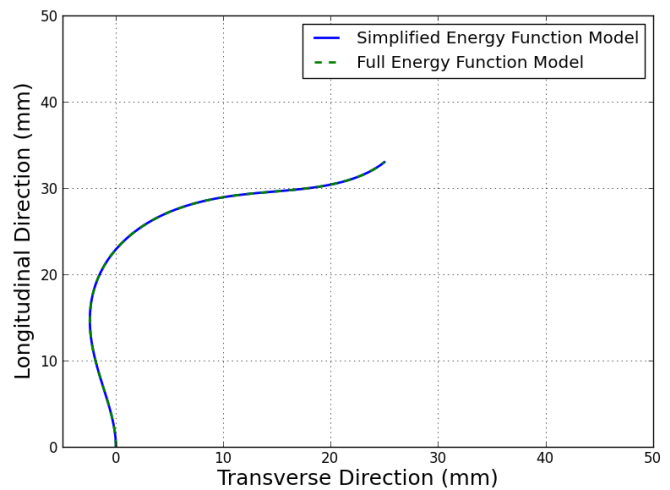

(c)

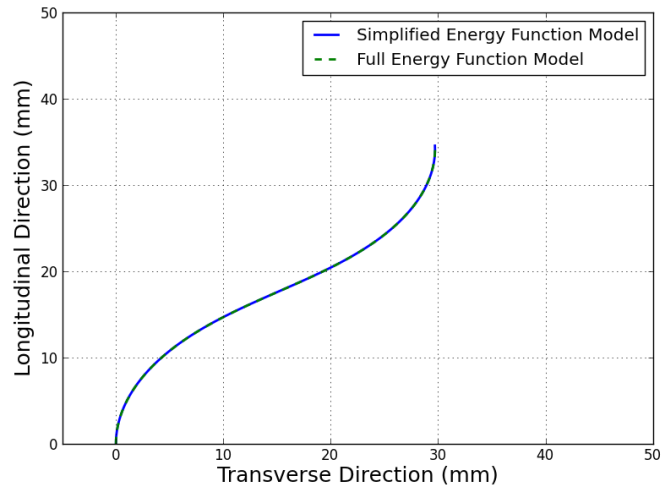

(e)

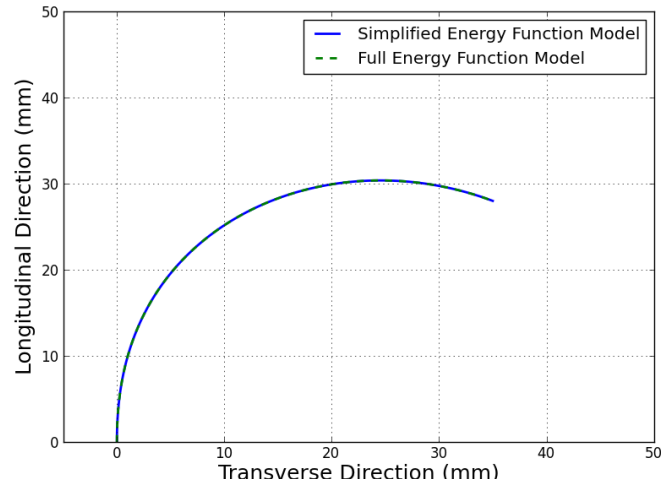

(b)

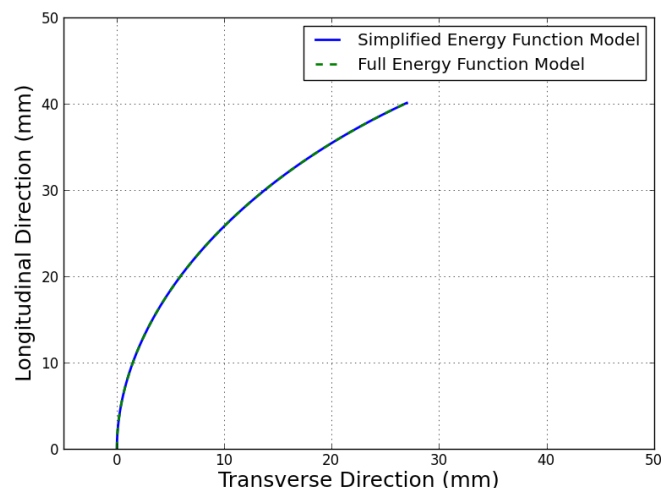

(d)

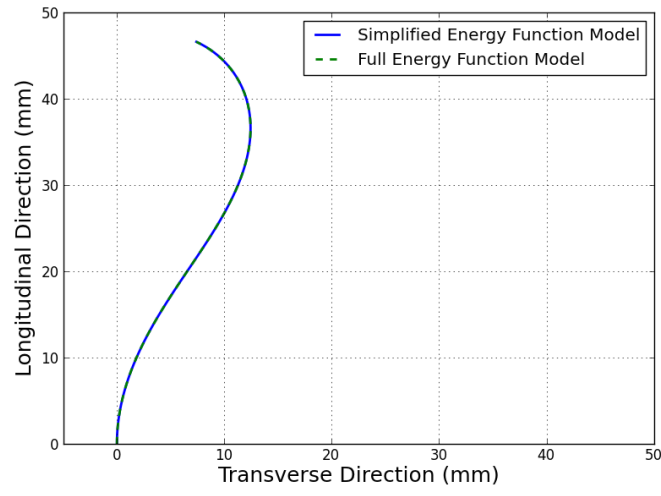

(f)

Figure 3.10: A set of six plots showing the output of the IFM using the simplified energy function (Eq. 3.20) compared against the IFM using the full energy function (Eq. 3.6). 
The beam shape predicted by the model using a sixth-order polynomial parameterization was then compared against the experimentally recorded beam shapes. Figure 3.11 shows comparisons between predicted and experimentally determined beam shapes for six unique sets of input position and orientation. For each set, the solid line represents the beam shape predicted by the model and the dots represent the corresponding experimentally determined beam shape.

A set of predicted beam shape solutions was also generated using the IFM with an eighth-order polynomial parameterization. These results were then compared against the experimental beam shapes and the sixth-order polynomial parameterization as shown in Fig. 3.12. For this comparison, the same end conditions found in Table 3.1 were used. For each set, the solid line represents the beam shape predicted by the sixth-order model, the dashed line represents the beam shape predicted by the eighth-order model, and the dots represent the corresponding experimentally determined beam shape. For each of the evaluated end coordinates, the eighth-order polynomial model produced nearly identical predictions to the sixth-order polynomial model.

As a point of comparison, the use of a linear spline parameterization for the beam shape was also explored and compared against the experimental results. These model predictions are shown in Fig. 3.13. The experimental results are again shown by dots while the spline models are shown as solid, dashed, dashdotted, and dotted lines for 6-segment, 8-segment, 10-segment, and 28-segment splines, respectively. As can be seen, the lower segment number spline models had difficulty producing a reasonable beam shape for some position and orientation sets, most notably Sub-Figs. (b) and (f).

A comparison between the sixth-order polynomial parameterization and the 28segment spline parameterization relative to the experimental beam shape is shown in Fig. 3.14. Here, the polynomial solution is depicted with a solid line while the spline solution is shown with a dashed line. 


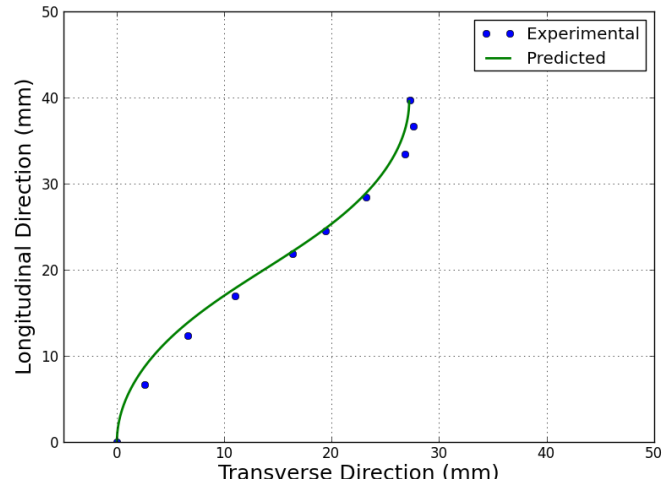

(a)

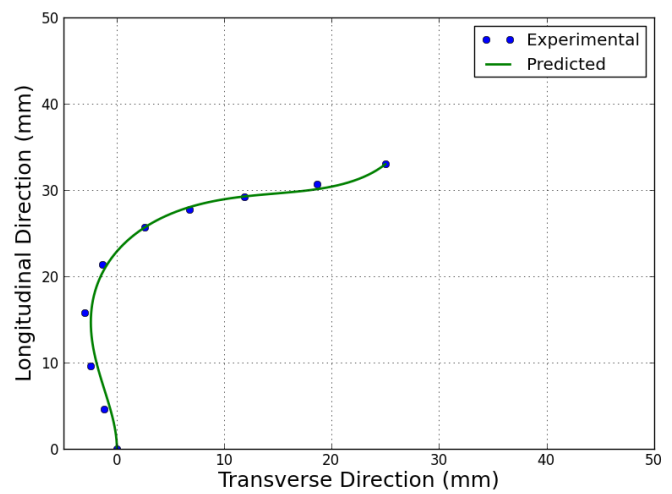

(c)

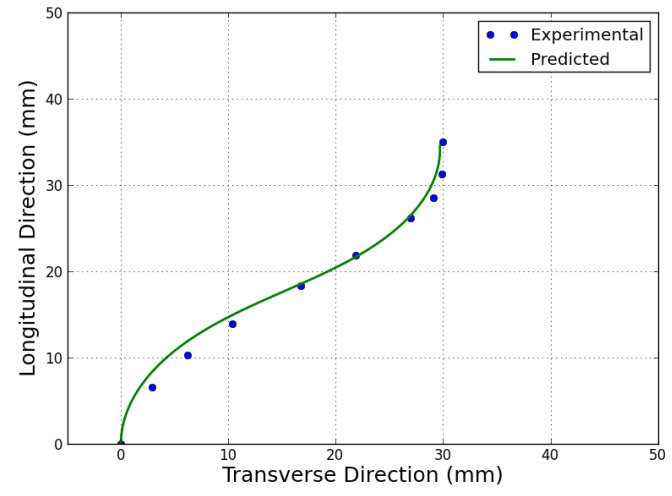

(e)

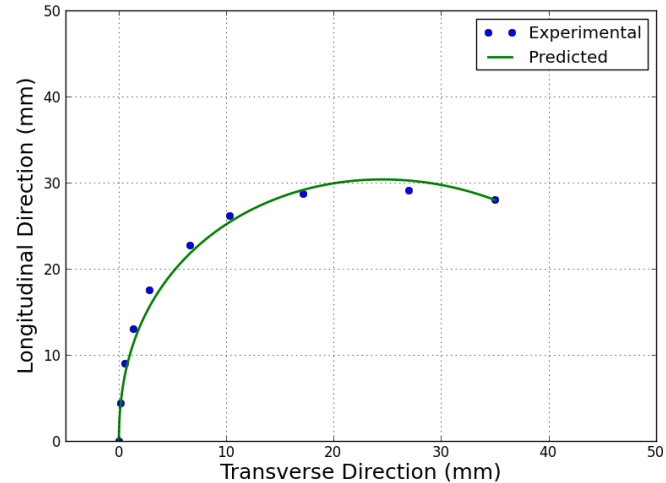

(b)

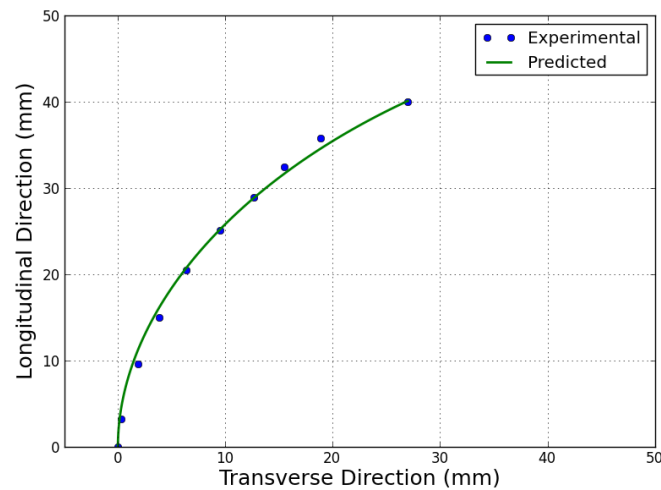

(d)

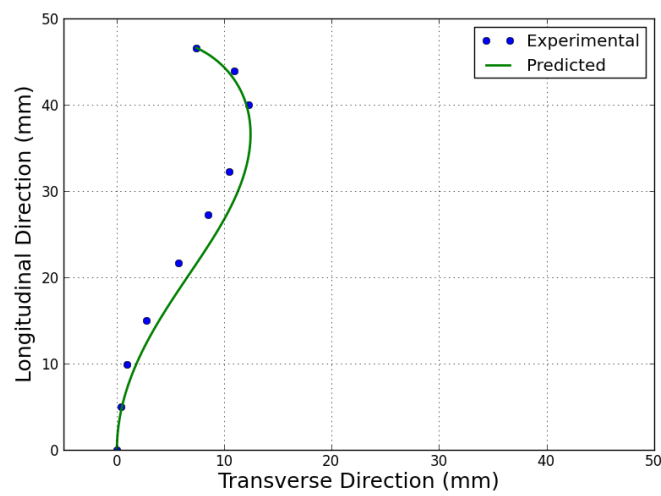

(f)

Figure 3.11: A set of six plots showing the output of the IFM with 6th order polynomial parameterization compared against the experimentally determined beam shape where $\left[x_{e n d}, y_{\text {end }}, \theta_{\text {end }}\right]$ correspond to the sub-figure references in Table 3.1 . 


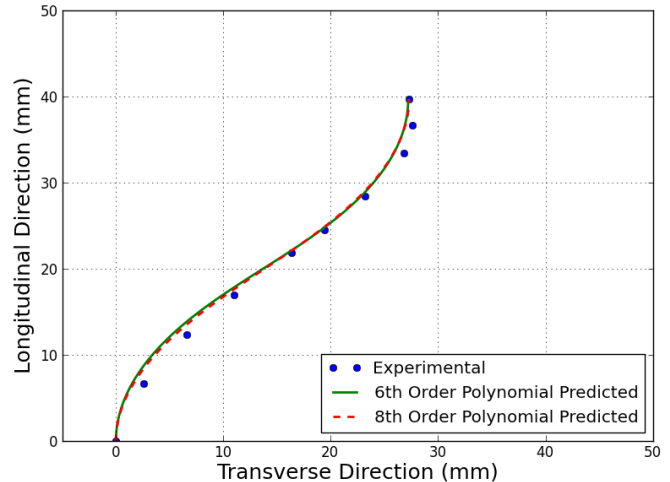

(a)

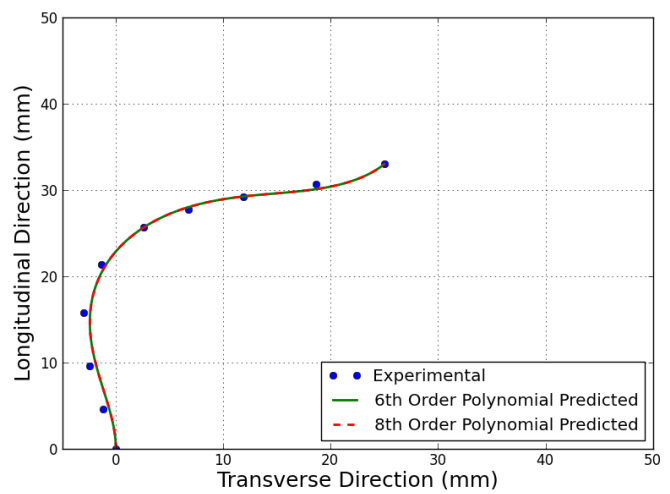

(c)

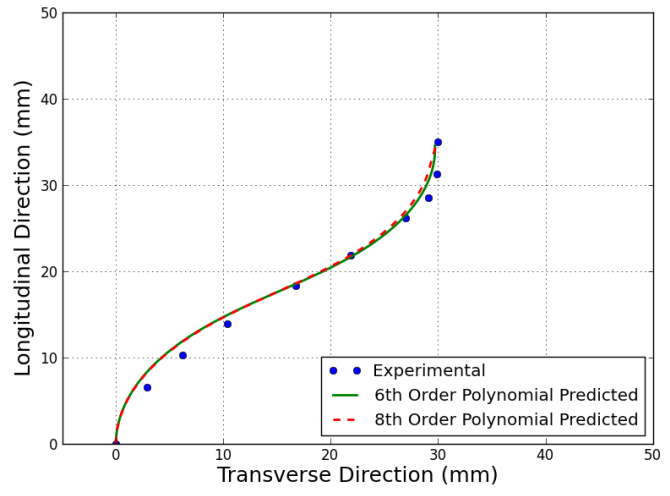

(e)

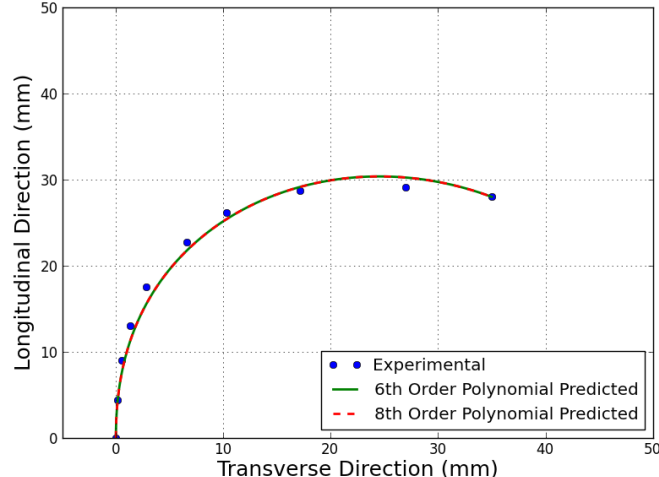

(b)

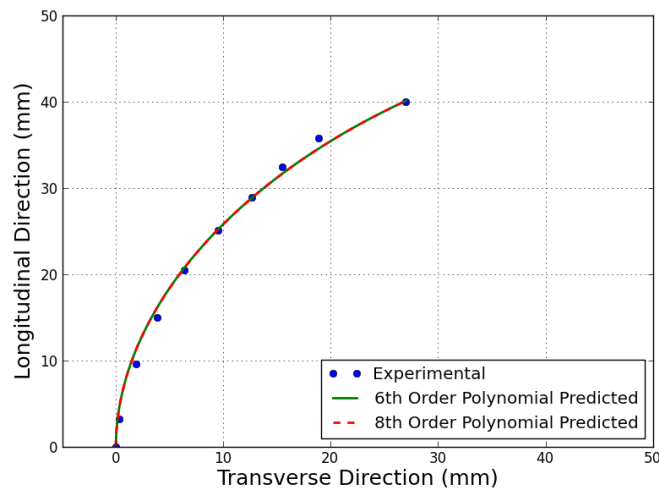

(d)

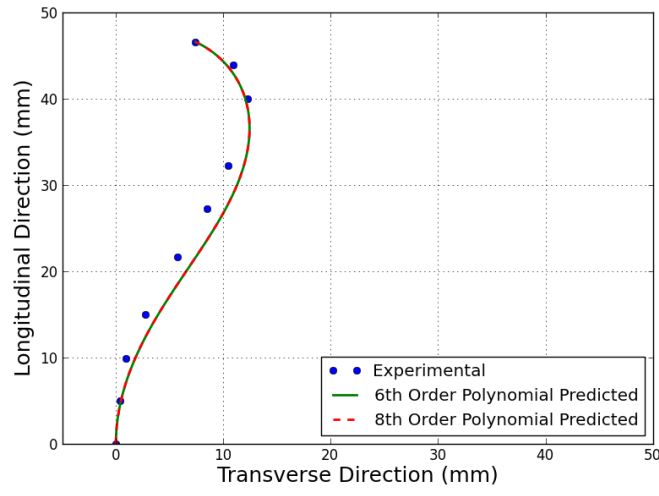

(f)

Figure 3.12: A set of six plots showing the output of the IFM using a 6th order polynomial, an 8th order polynomial, and the experimentally determined beam shape where $\left[x_{\text {end }}, y_{\text {end }}, \theta_{\text {end }}\right]$ correspond to the sub-figure references in Table 3.1 . 


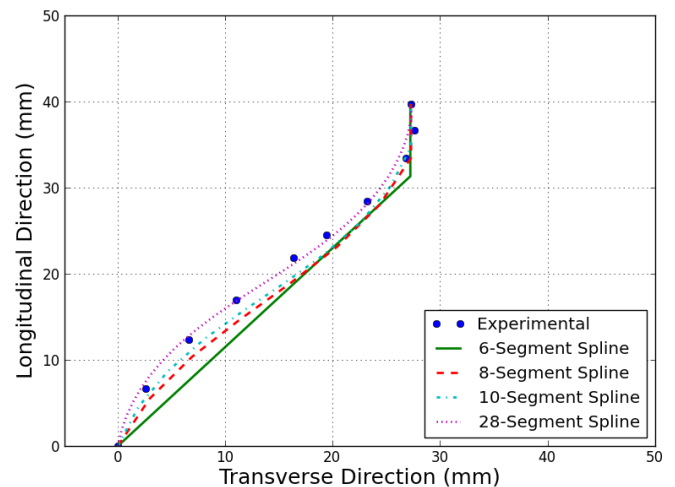

(a)

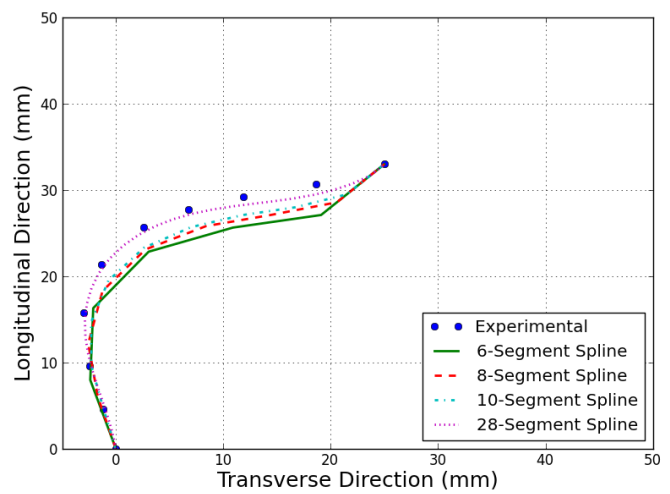

(c)

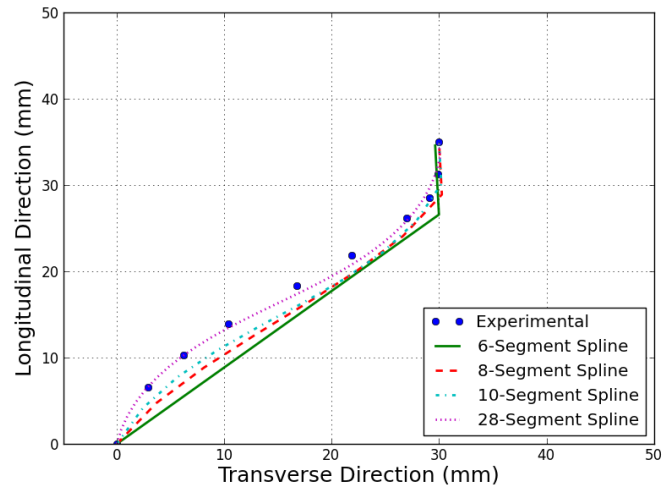

(e)

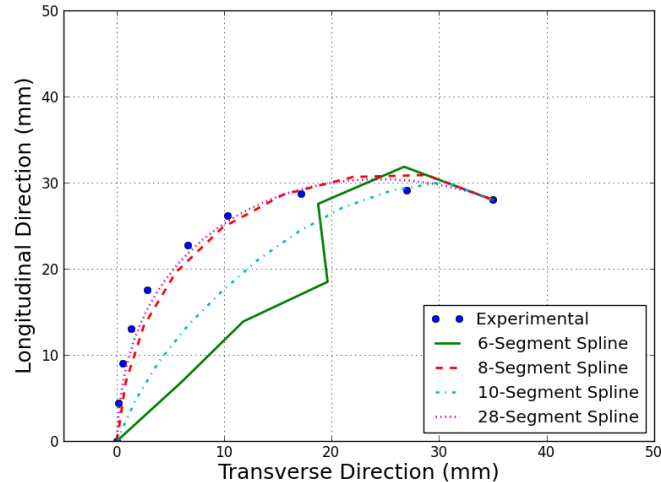

(b)

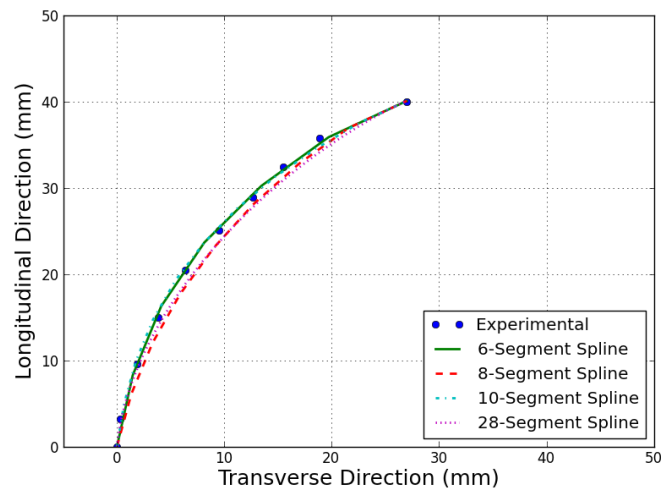

(d)

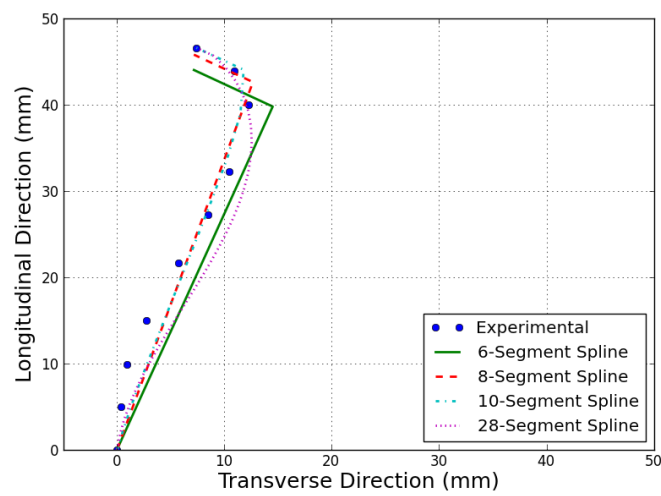

(f)

Figure 3.13: A set of six plots showing the output of the IFM using spline parameterizations. 6-segment, 8-segment, 10-segment, and 28-segment. [ $\left[x_{\text {end }}, y_{\text {end }}, \theta_{\text {end }}\right]$ correspond to the sub-figure references in Table 3.1 


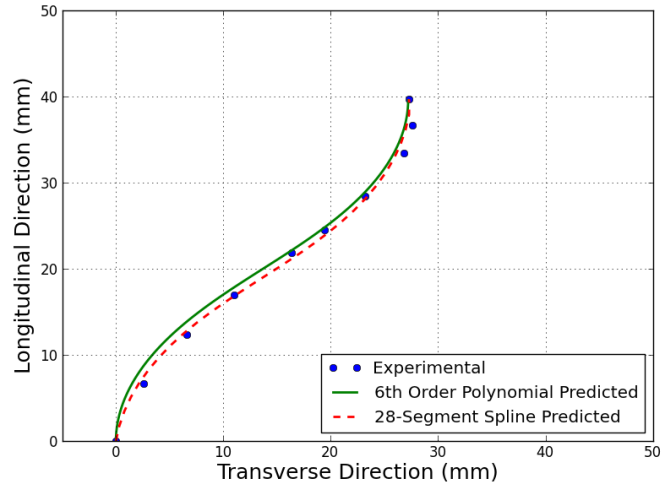

(a)

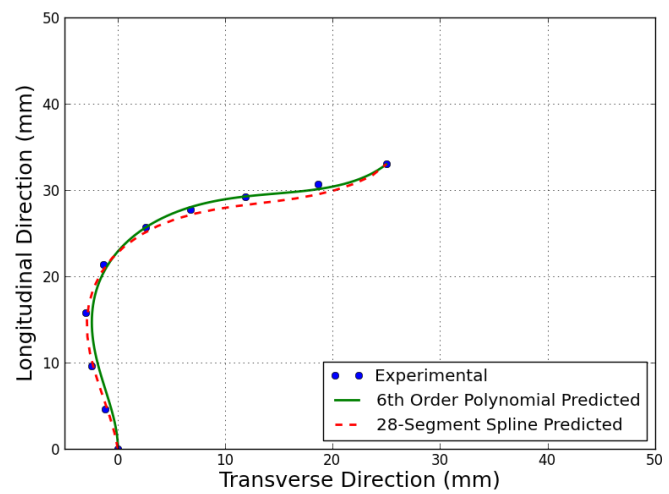

(c)

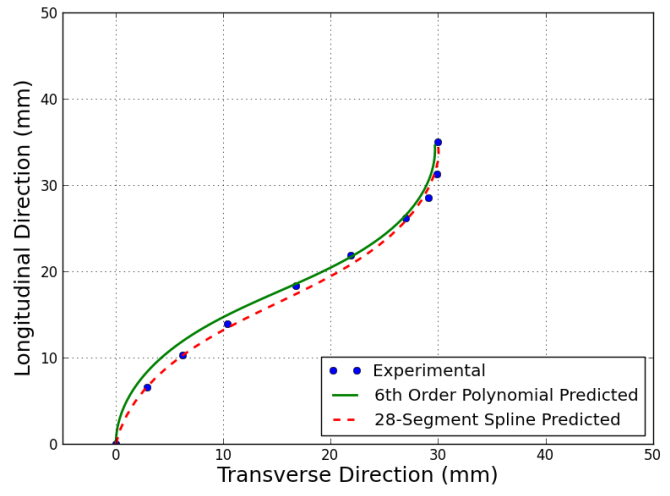

(e)

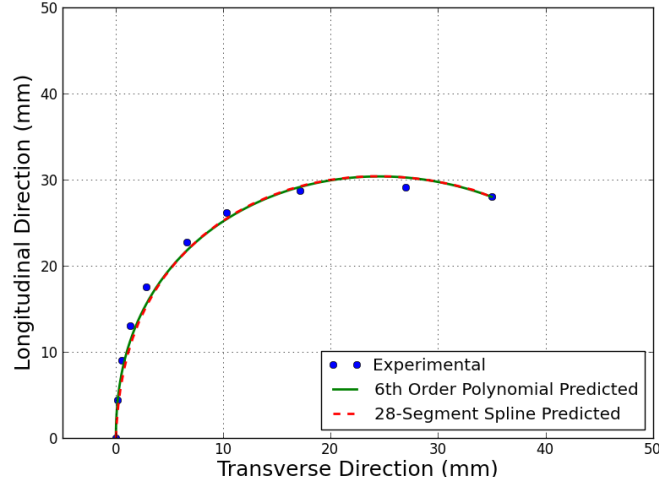

(b)

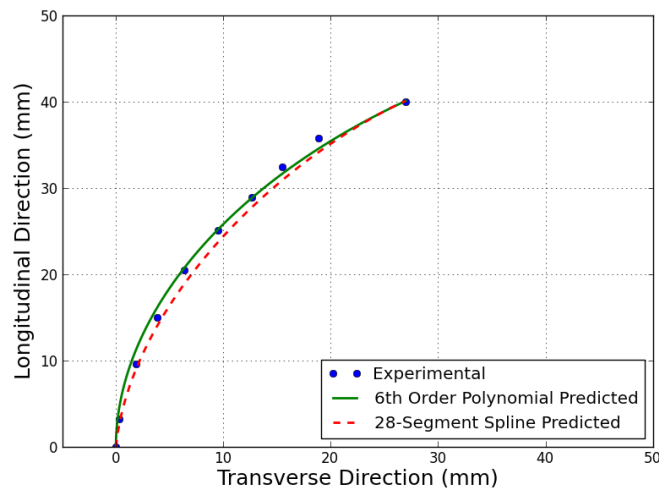

(d)

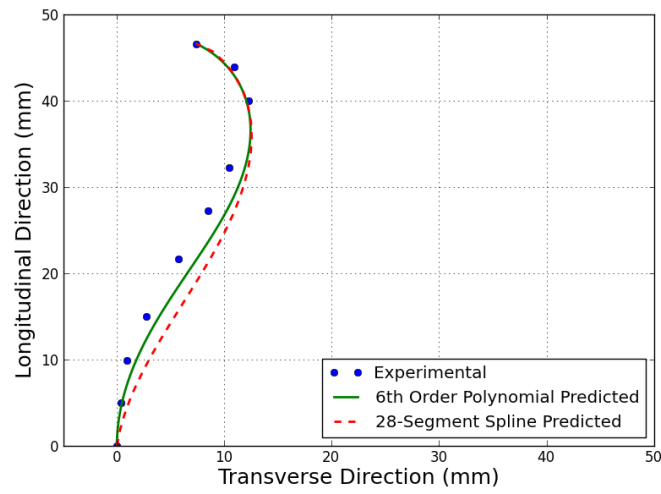

(f)

Figure 3.14: A set of six plots comparing the output of the IFM using 6th order polynomial parameterization versus the 28 -segment spline parameterization. $\left[x_{\text {end }}, y_{\text {end }}, \theta_{\text {end }}\right]$ correspond to the sub-figure references in Table 3.1 . 
A comparison of the spline predicted end loads with both the polynomial predicted and experimentally determined end loads is also shown in Table 3.3. The load applied to the distal end of the beam was recorded using the six-axis load cell. As this experiment was conducted using the planar (two-dimensional) case, only $F_{z}, F_{x}$, and $M_{y}$ loads are shown. The load cell was oriented such that the $\mathrm{y}$-component of the force and the $\mathrm{x}$ - and $\mathrm{z}$-components of the torque were zero. Thus only three of the possible six measurements from the load cell were used. These results are represented graphically in Fig. 3.15. The results show quite a bit of variability between the parameterization methods. The error between the experimental and model predicted end loads for both the sixth order polynomial and 28-segment spline parameterizations are plotted against the strain energy calculated from the polynomial parameterization for each of the tested experimental conditions. The strain energy used here is the optimized strain energy as calculated using the method demonstrated in Section 3.3.1. There is some observed dependence between the absolute force calculation errors and the calculated strain energy. However, on a percent error basis, this trend is not as clear indicating that additional sources of error such as experimental measurement or calibration error are likely to be significant. Further, qualitatively it was observed that little variation was present between parameterization order with the exception of the lower segment number spline methods which occasionally produced largely different force and moment predictions.

To provide a means of comparing the relative quality of the model predictions, it was necessary to develop a metric for this measure based upon the coefficient of determination. The coefficient of determination itself is a measure of the goodness of fit between a dataset and a prediction of those values [124]. Typically, the coefficient of determination $\left(R^{2}\right)$ is calculated using the ratio of the residual error between the dataset and the model (unexplained variation) to the total variation of the dataset using one dependent variable and one independent variable. Similarly, the coefficient of multiple determination calculates the same value but now with 
Table 3.3: Experimental versus model predicted end loads for a sixth-order polynomial model and a 28-segment spline model. Forces are given in $\mathrm{N}$ and moments in $N \cdot m$.

\begin{tabular}{c|ccc|ccc|ccc}
\hline Sub- & \multicolumn{3}{|c|}{ Polynomial Predicted } & \multicolumn{3}{c|}{ Spline Predicted } & \multicolumn{4}{c}{ Experimental } \\
Fig. & $F_{z}$ & $F_{x}$ & $M_{y}$ & $F_{z}$ & $F_{x}$ & $M_{y}$ & $F_{z}$ & $F_{x}$ & $M_{y}$ \\
\hline (a) & 0.13 & -0.11 & 0.0039 & 0.17 & -0.04 & 0.0031 & 0.10 & -0.02 & 0.007 \\
(b) & 0.02 & 0.07 & -0.0020 & 0.04 & 0.09 & -0.0017 & 0.05 & 0.06 & -0.05 \\
(c) & 0.57 & 0.38 & 0.0054 & 0.50 & 0.32 & 0.0040 & 0.28 & 0.15 & 0.20 \\
(d) & 0.01 & -0.02 & -0.0007 & 0.05 & -0.02 & -0.0015 & 0.04 & -0.01 & 0.00 \\
(e) & 0.27 & -0.03 & 0.0045 & 0.21 & -0.04 & 0.0006 & 0.15 & 0.00 & 0.06 \\
(f) & 0.18 & -0.24 & 0.0066 & 0.13 & -0.12 & 0.0047 & 0.06 & -0.05 & 0.10 \\
\hline
\end{tabular}

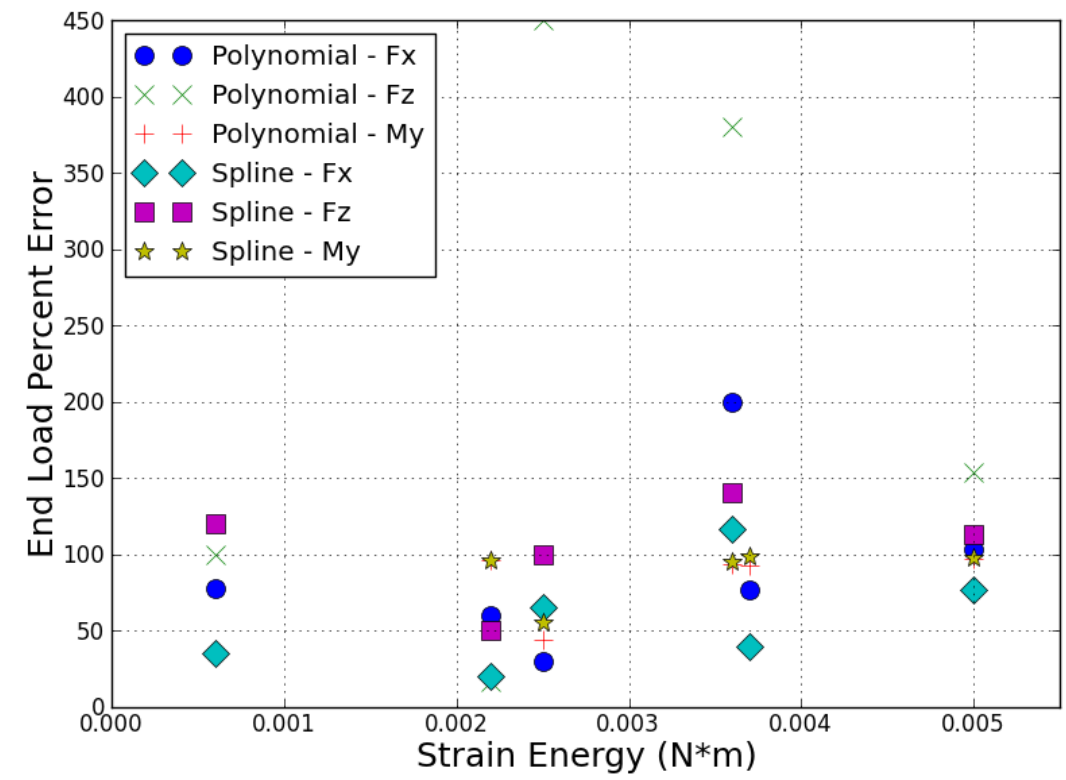

Figure 3.15: Error between experimental and model predicted end loads for both 6th order polynomial and 28-segment spline parameterizations plotted against strain energy calculated from the 6th order polynomial parameterization for each of the tested experimental conditions. 
two independent variables and one dependent variable. However, as a means of calculating the goodness of fit between the IFM prediction for the beam shape and the experimental data, it was desired to parameterize the calculation on the basis of the arc position, $s$. As such, the standard $R^{2}$ calculation was modified to account for variation in both coordinate directions.

Under these conditions, the $R^{2}$ value was calculated as

$$
R^{2}=1-\frac{S S_{r e s}}{S S_{t o t}}
$$

where $S S_{\text {res }}$ is the residual sum of squares (Eq. 3.43) and $S S_{\text {tot }}$ is the total sum of squares (Eq. 3.44).

$$
\begin{aligned}
& S S_{\text {res }}=\sum_{i} \epsilon_{i}^{2} \\
& S S_{\text {tot }}=\sum_{i} P_{i}^{2}
\end{aligned}
$$

Here, $\epsilon_{i}$ represents the residuals (Euclidean distance) between a data point and the corresponding point on the beam curve predicted by the model as shown in Fig. 3.16. The value for $\epsilon_{i}$ was found by first determining the position, $s$, along the beam matched to each data point. Then the location of the corresponding position, $s$, on the model predicted beam was found. The distance between these points was computed and used to represent the residual for each data point. The value $P_{i}$ represents the Euclidean distance between each data point and the average of the location for all the points within the dataset, also shown in Fig. 3.16 .

Using this calculation for the $R^{2}$ value, the sixth order polynomial and the 28segment spline parameterizations were compared. The results are shown in Table 3.4. along with values for the total sum of the residuals for all of the data points as well as the maximum calculated residual. The output of the model for each parameterization is plotted in Fig. 3.14 for each of the corresponding experimental 


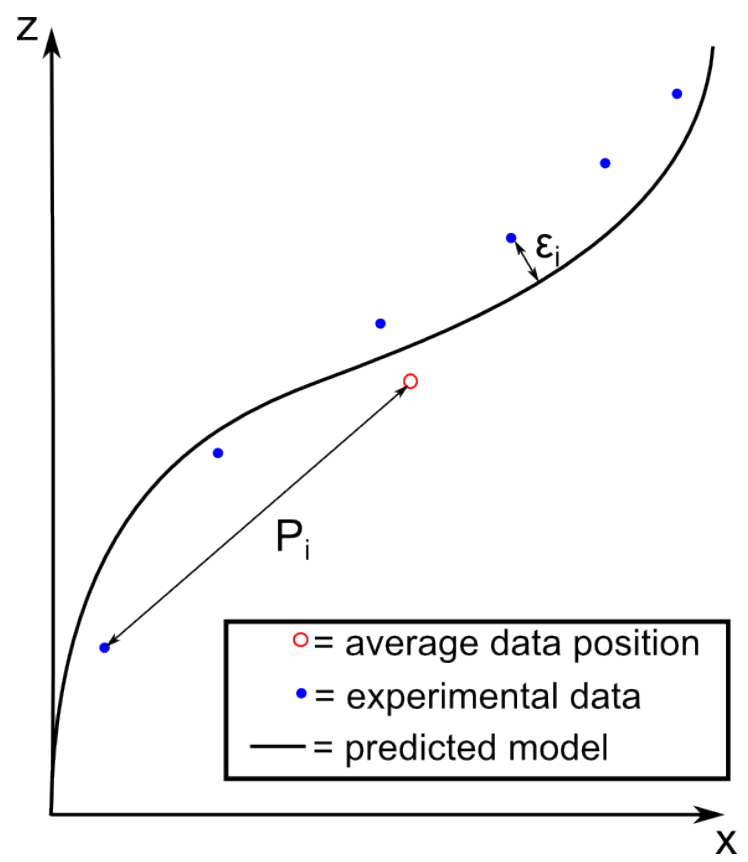

Figure 3.16: Graphic illustrating the variables used in the calculation of the coefficient of determination.

conditions. It can be seen that the model achieved $R^{2}$ values greater than 0.98 with either parameterization for each of the experimental conditions.

Table 3.4: Coefficient of determination, total residual error, and maximum residual error calculated for each sub-figure. Values presented for both the sixth order polynomial and 28-segment spline parameterizations. Parameterization with higher $R^{2}$ value for each dataset is indicated by boldface type.

\begin{tabular}{c|ccc|ccc}
\hline Sub- & \multicolumn{2}{|c|}{6 th Order Polynomial } & \multicolumn{3}{|c}{$28-$ Segment Spline } \\
Fig. & $R^{2}$ & $\epsilon_{\text {tot }}$ & $\epsilon_{\max }$ & $R^{2}$ & $\epsilon_{\text {tot }}$ & $\epsilon_{\max }$ \\
\hline (a) & 0.99639 & 6.85 & 1.23 & $\mathbf{0 . 9 9 7 4 1}$ & 5.33 & 1.15 \\
(b) & $\mathbf{0 . 9 9 7 9 9}$ & 5.07 & 1.10 & 0.99676 & 6.97 & 1.19 \\
(c) & $\mathbf{0 . 9 9 8 6 5}$ & 3.90 & 0.63 & 0.99525 & 7.51 & 1.26 \\
(d) & $\mathbf{0 . 9 9 8 8 6}$ & 3.62 & 0.93 & 0.99642 & 6.98 & 1.28 \\
(e) & 0.99533 & 8.10 & 1.37 & $\mathbf{0 . 9 9 7 5 9}$ & 5.15 & 1.19 \\
(f) & $\mathbf{0 . 9 9 5 0 4}$ & 7.76 & 1.64 & 0.98401 & 14.65 & 3.03 \\
\hline
\end{tabular}


While there is no clear consensus on what constitutes an acceptable $R^{2}$ value, it is possible to use it as a means of comparision between the different experimental conditions and the parameterizations. From Table 3.4, it is observed that each parameterization achieved a higher $R^{2}$ value than the other on some of the experimental conditions as indicated by the boldface type. This suggests that each parameterization is roughly equivalent in terms of prediction accuracy. Therefore, there is no clear benefit in terms of predicting the beam shape to using the 28-segment spline over the sixth order polynomial since the former requires significantly more effort to compute than the latter.

\subsection{Summary of Manipulator Backbone Model}

The alternative beam model presented in this section has shown potential as a relatively simple method for predicting the shape of a continuum beam manipulator when driven to a prescribed tip location and orientation. The model uses inverse kinematics to calculate the potential energy stored within the beam and then selects the beam shape with the lowest potential energy as the most likely solution among the set of possible beam shape solutions. These calculations are performed under the assumption that all externally applied loads occur at the distal end of the manipulator and that strain due to bending and torsion of the beam are the primary contributors to the total distortion energy stored within the beam. Additionally, the model neglects the effects of the beam mass itself and thus care should be taken if the length to diameter ratio of the manipulator backbone is particularly large. As the backbone diameter is decreased, the manipulator length would also need to be reduced in order to prevent large tip deflections due to the beam mass.

The results shown in the previous section demonstrate that the IFM does a reasonable job at predicting beam shape for all of the beam tip configurations tested 
with a maximum observed residual error of $3.03 \mathrm{~mm}$ in the most extreme case. The model exhibited some difficulty in predicting beam shape for some of the tested conditions. However, as shown in Fig. 3.10, these errors did not change with or without the inclusion of axial and transverse strains. Thus it is likely that these errors can be attributed to experimental measurement error and errors in the estimation of the material properties. The performance of the IFM when using an eight degree polynomial was also evaluated as shown in Fig. 3.12. Here it was found that the eight degree parameterization did not perform significantly better than the six degree parameterization when it came to predicting beam shape. This would suggest that there is little benefit to using the higher order parameterization since it comes at the cost of computational effort and produces no consistent gains in accuracy.

Next, a spline parameterization was explored as an alternative to the polynomial solution method. The overall observation, as expected, is that the accuracy of the spline model when predicted the experimental beam shape improves as the number of segments increases as shown in Fig. 3.13. For each of the tested end locations corresponding to Sub-Figs. (a)-(f), the spline model was found to converge with $14,18,19,6,11$, and 20 segments, respectively. Further, with the exception of the positions in Figs. $3.14 \mathrm{e}$ and $3.14 \mathrm{f}$, the spline model converged to the beam shape predicted by the polynomial model. It is also noted that except in the higher curvature positions, the 6 -segment spline model did a reasonable job at predicting the beam shape. The 28 -segment spline model produced beam shape predictions that are better than the polynomial model for some positions and worse for other positions, as shown in Table 3.4. No clear trend indicating which model better predicted the solution was apparent from these results. Overall, the beam shape predictions produced by the IFM demonstrated reasonable accuracy for the majority of tested boundary conditions by either of the tested parameterization methods. While the spline parameterization method may be capable of producing better results under some circumstances, this method typically 
required significantly more free variables to produce a viable solution. Possible sources of error in this investigation include measurement error for the experimentally determined beam position and orientation as well as errors stemming from the tolerance built into the optimization algorithm (set to $1 \mathrm{~mm}$ and $0.057^{\circ}$, for position and orientation respectively). These tolerance settings were appropriate as the IFM predictions were found to be relatively insensitive to small changes in the position and orientation boundary conditions; meaning that changing the boundary conditions did not drastically change the shape of the resulting beam prediction.

The data presented in Table 3.3 shows the predicted end loads versus the experimentally measured end loads. Predicted end load data was calculated using both the sixth-order polynomial parameterization and the 28-segment spline parameterization. It was found that the IFM predicted results for the beam forces at the tip for a given beam shape did not correlate well with the experimental measurements when using either method of parameterization. The spline model produced slightly better predictions for some end loads, but this result was not observed consistently. Better end load predictions from the spline model are expected since the spline model uses more variables to produce a solution and thus has more flexibility. It should also be noted that limitations of the experimental measurement hardware likely contributed to errors in the force and torque measurements since the loads being measured were near the lower end of the limits for accurate measurement for the six-axis load cell (estimated resolution of 0.063 $\mathrm{N}$ and $0.0008 \mathrm{~N} \cdot \mathrm{m}$ for force and moment measurements, respectively). Future efforts should work towards producing a more robust validation of the end load predictions using measurement equipment with greater precision. 


\section{Chapter 4}

\section{Artificial Muscle Actuation Unit}

A robot for any purpose requires some method of introducing power into the system, often in the form of an actuator (e.g. motor driven, piston and cylinder, or artificial muscle based). Thus, this chapter first provides an overview of the available actuation options and rationale for the selection of the artificial muscle actuator in Section 4.1. Section 4.2 provides the mathematical description of the muscle actuator and is followed by a discussion of the specification process for the actuator parameters and the analysis that contributed to it in Section 4.3. Experimental results are presented in Section 4.4 to validate the appropriateness of the models and justify the selection of the actuator.

\subsection{Motivation and Related Work}

There are a variety of methods available to achieve the controllable linear actuation necessary to articulate the segments that allow for positioning of the distal end of the device. Some of the possibilities include electromechanical linear actuators, pneumatic actuators, or hydraulic actuators. A comparison of these three 
choices reveals that each option has both advantages and disadvantages. Electromechanical actuators are typically considered to be more efficient than their pneumatic and hydraulic counterparts, while hydraulic actuators have the highest power to weight ratio of the three choices presented here, assuming that the hydraulic power source is located remotely [75]. By prioritizing the design constraints of the present device, it was determined that the size restriction due to the nature of natural orifice surgery was the most critical condition to be met, followed by the force output from the device that is necessary to achieve desired performance. Therefore, hydraulic actuation was chosen for its higher power output despite any possible lack in efficiency, which is less critical in surgical applications.

Although hydraulic actuation offers high power density, mechanical rigidity and high dynamic response, the force capability, $F$, for a given pressure, $P$, of a conventional linear hydraulic actuator is limited by the piston area, $A$, given by: $F=P \cdot A$. In view of the stringent diametric requirement of the present application, the use of hydraulic artificial muscle actuators (AMA) (see Fig. 4.1), which will be shown to have better peak force capability than conventional linear actuators for the same pressure and diameter, will be explored [125]. While the concept of artificial muscle actuators and their use in robotics is not new, the majority of applications use pneumatic power to drive them [57, 68, 126, 127]. However, through the use of a hydraulic medium it may be possible to mitigate some of the issues with responsiveness and rigidity that have been encountered previously. There are two important advantages to a hydraulic approach: 1) a higher pressure (by a factor of 10 compared with pneumatic) can be applied so that force and power density can be further increased and the actuator diameter can be decreased; 2) liquid has a much lower compressibility and therefore better rigidity than compressed gas.

Artificial muscle actuators consist of a contained internal bladder, surrounded by a flexible, braided outer sheath. It is the geometry of this outer sheath that transmits radial expansion of the internal bladder due to an applied pressure to 
contractile force along the longitudinal axis of the muscle actuator [128]. As the radius of the bladder, and thus the outer mesh, increases, the individual strands of the mesh which are woven in an over-under crossing pattern rotate relative to each other and to the long axis of the actuator and shorten the longitudinal distance from one end of the strand to the other. The load capacity of the artificial muscle actuator is then a function of the geometry and orientation of the outer sheath and the pressure applied to the internal bladder [129]. As discussed in Section 6.2 , it is the contraction of the AMA which provides input positioning load to the manipulator through the application of tension to the attached tendon.

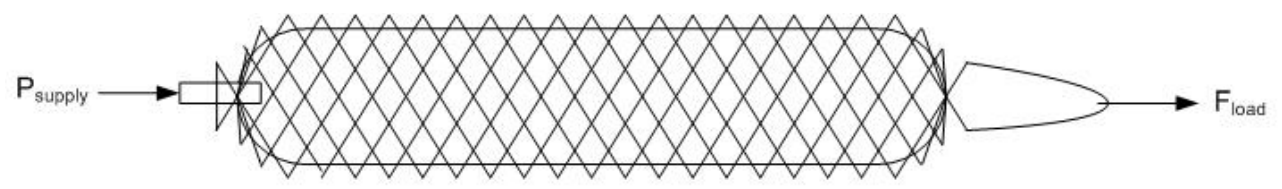

Figure 4.1: Artificial muscle actuator.

\subsection{Analytical Description of Artificial Muscle Actuators}

There are two methods presented in the literature for modeling the transmission of internal pressure to contractile force of an AMA. The first is a theoretical approach based upon energy conservation [125], while the second is an examination of the force profile of the surface pressure [130]. The first approach is based on the principle that energy supplied to the actuator by the pressurized fluid must leave the actuator through the application of a load over some distance. The second approach is based on an examination of the distortion of the internal bladder under isobaric conditions. However, ultimately each of these methods arrives at the base model [127]. A summary of the first method is presented here. It should be noted that this model does not account for possible effects of compressibility 
in the bladder, interactions between the bladder and the braided outer sheath, or any contribution from the ends of the muscle actuator.

As shown in Fig. 4.2, adapted from Chou and Hannaford [129], it is first necessary to define the geometric parameters of the braided sheath. These parameters include the length of the individual braided strands, $b$, the number of turns each strand makes over the length of the actuator, $n$, and the angle between the strands and the longitudinal axis of the actuator, $\gamma(t)$, which is assumed to be uniform for all strands within the braided sheath.

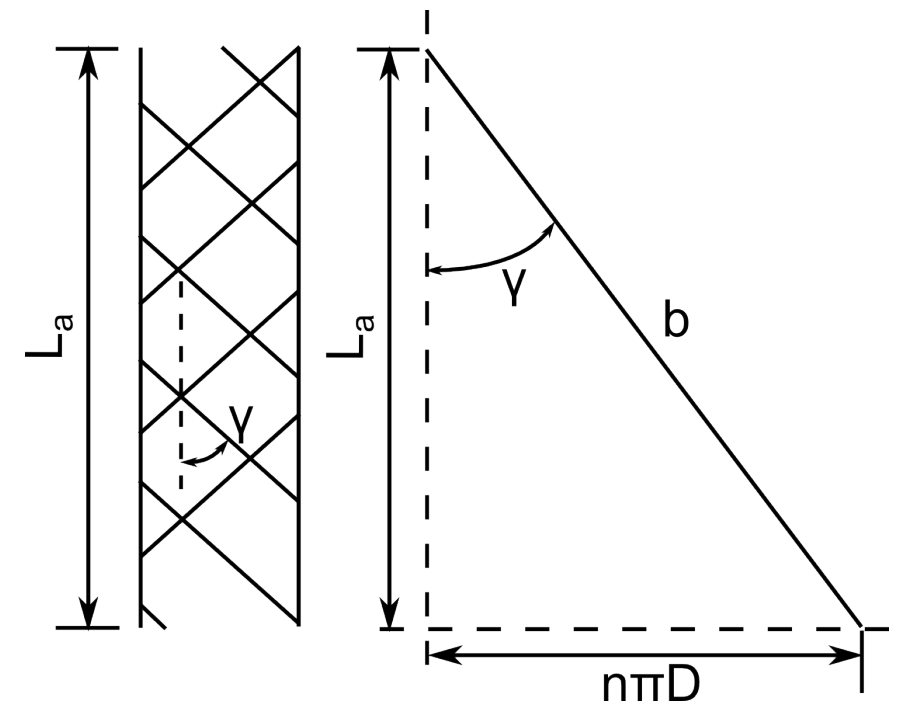

Figure 4.2: Artificial muscle actuator geometry (left) and magnified view of braid geometry relationship for a single unwound strand (right).

The overall length of the actuator, $L_{a}(t)$, and the actuator diameter, $D(t)$, can then be represented in terms of the constants, $n$ and $b$, and as functions of the braid angle, $\gamma(t)$, as seen in Eq. 4.1) and Eq. (4.2).

$$
\begin{aligned}
& L_{a}(t)=b \cdot \cos \gamma(t) \\
& D(t)=\frac{b \cdot \sin \gamma(t)}{n \pi}
\end{aligned}
$$


Then, calculating the volume of a cylinder and substituting in the functions for $L_{a}(t)$ and $D(t)$,

$$
V(t)=\frac{\pi}{4} D(t)^{2} L_{a}(t)=\frac{b^{3}}{4 \pi n^{2}} \cdot \sin ^{2} \gamma(t) \cos \gamma(t)
$$

The first derivatives of $L_{a}(t)$ and $V(t)$ with respect to $\gamma(t)$ are calculated as

$$
\begin{gathered}
\frac{d L_{a}(t)}{d \gamma(t)}=-b \cdot \sin \gamma(t) \\
\frac{d V(t)}{d \gamma(t)}=\frac{b^{3} \sin \gamma(t)}{2 \pi n^{2}} \cdot\left(\cos ^{2} \gamma(t)-\frac{1}{2} \sin ^{2} \gamma(t)\right)
\end{gathered}
$$

From Eq. (4.4) and Eq. (4.5), the first derivative of $V(t)$ with respect to $L_{a}(t)$ is given as

$$
\frac{d V(t)}{d L_{a}(t)}=\frac{d V(t) / d \gamma(t)}{d L_{a}(t) / d \gamma(t)}=\frac{-b^{2}}{4 \pi n^{2}} \cdot\left(3 \cos ^{2} \gamma(t)-1\right) .
$$

From the principle of virtual work we have:

$$
F_{a}(t) \cdot d L_{a}(t)=P_{a}(t) \cdot d V(t)
$$

and solving Eq. 4.7) for the force output with Eq. (4.1 and 4.6) results in

$$
\begin{aligned}
F_{a}(t) & =P_{a}(t) \frac{b^{2}}{4 \pi n^{2}} \cdot\left[3 \frac{L_{a}(t)^{2}}{b^{2}}-1\right] \\
& =P_{a}(t) \frac{\pi D_{0}^{2}}{4} \cdot\left[3 \cos ^{2}(\gamma(t))-1\right]
\end{aligned}
$$

where $F_{a}(t)$ is the contractile force, $P_{a}(t)$ is the pressure differential across the bladder wall, and $D_{0}=\frac{b}{\pi n}$ (obtained by evaluating Eq. 4.2 at $\gamma=90^{\circ}$ ) is the theoretical maximum muscle diameter. Note that the strand number does not factor into the calculation of the contraction force. This is because this relationship is derived purely from the geometry of a single strand during a muscle contraction and thus does not change with strand number. 
Examining the term within the brackets in Eq. (4.9) and observing that it can be greater than $1\left(\lim _{\gamma(t) \rightarrow 0}=2\right)$, the force capability of an artificial muscle can be greater than that of a hydraulic piston actuator for the same diameter, $D_{0}$, and pressure. However, this comparison is based on a maximum AMA diameter occurring at $\gamma=90^{\circ}$. If $F_{a}(t)$ is set to zero, we see from Eq. 4.9 that $\gamma(t)=$ $54.7^{\circ}$. This represents the maximum achievable contraction of the muscle meaning that larger braid angles are not achievable. Thus this maximum value for $\gamma$ is independent of all other parameters and holds true under all conditions. This represents the true point of maximum contraction and corresponding maximum AMA diameter, $D_{\max }$. . Substituting this diameter into Eq. 4.9, it is found that the AMA force can be up to three times that of a similar hydraulic piston actuator as shown in Eq. 4.10.

$$
F_{a}(t)=P_{a}(t) \frac{\pi D_{\max }^{2}}{4} \cdot\left[\frac{3 \cos ^{2}(\gamma(t))-1}{\sin ^{2}\left(54.7^{\circ}\right)}\right]
$$

It is assumed here that the force from the hydraulic piston actuator is $F=P$. $A$ where the diameter is equivalent to the maximum diameter of the AMA. In reality the available area may be reduced by the piston rod cross-sectional area. Notice, however, that this advantage comes at the cost of having the force/pressure relationship vary with $\gamma(t)$ or actuator length $L_{a}(t)$, illustrated in Fig. 4.3. At the moment when the braid angle is $41.8^{\circ}$, the two actuators have equivalent load capacities.

Refinements to this basic model have been presented in the literature which include considerations for bladder end cap geometry, non-linear bladder material response, and frictional effects [126, 127, 129]. However, the basic model has been experimentally shown to provide sufficient accuracy to gain an understanding of the theoretical system performance without introducing unnecessary model complexity [131]. 


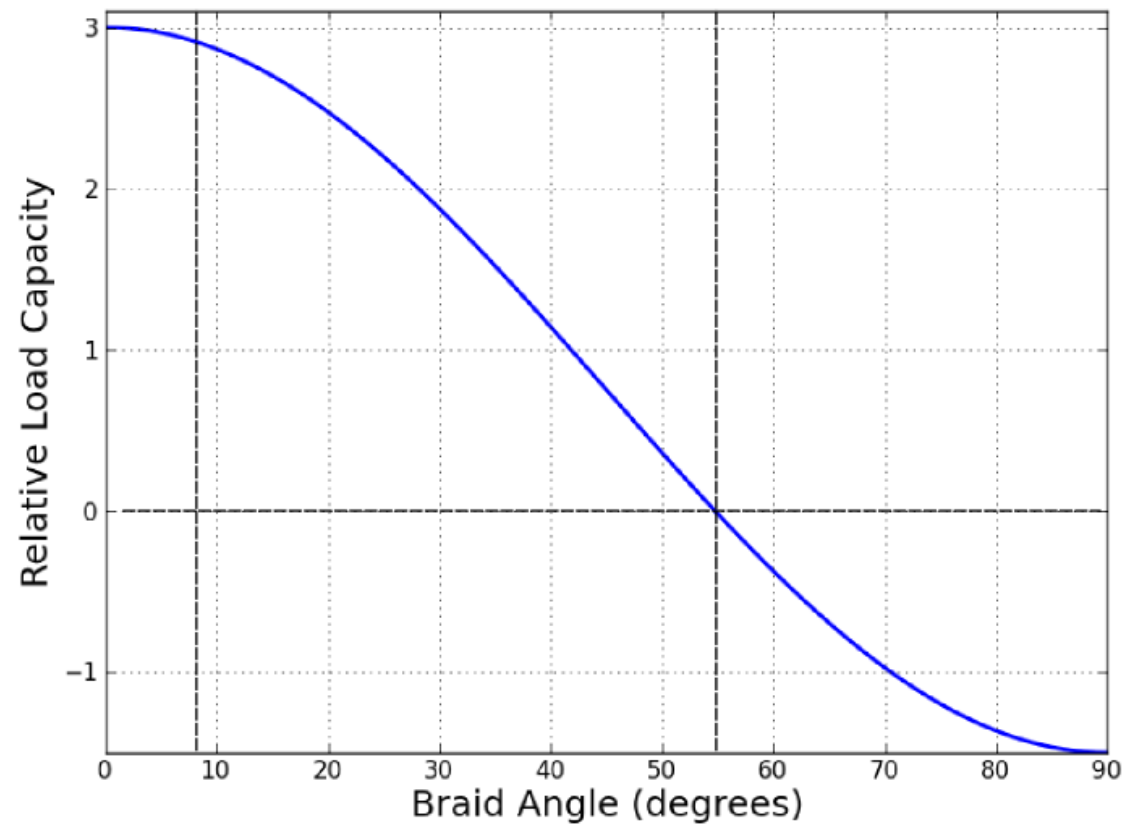

Figure 4.3: Ratio of force capacity for artificial muscle actuator to force capacity of a piston and cylinder when the maximum cross-sectional area of each actuator is equivalent $\left(F=P \cdot A_{\max }\right)$ as a function of braid angle. For the artificial muscle actuator, the maximum diameter is the diameter of the muscle at $\gamma(t)=54.7^{\circ}$.

The application of the AMA for use in driving a manipulator often involves arranging multiple actuators in an antagonistic fashion [128. The result is that when one actuator is activated, the corresponding antagonistic actuator must stretch beyond its nominal length to accommodate. Under these conditions the antagonistic actuator not only produces a contraction load in response to its internal pressure according to Eq. 4.8 but also a force in response to the elastic stretching of the inner bladder. In order to account for this, the elastic stretch of the internal bladder is modeled as a linear spring force such that $F_{s}=k \cdot \Delta L_{a}$, where $k$ is the spring constant and $\Delta L_{a}$ is the change in length of the internal bladder beyond its undeformed state. Thus, Eq. 4.9 is modified to account for elastic stretch of the inner bladder when the actuator is elongated beyond its nominal length as 
shown in Eq. 4.11.

$$
F_{a}(t)= \begin{cases}P_{a}(t) \frac{\pi D_{0}^{2}}{4} \cdot\left[3 \cos ^{2}(\gamma(t))-1\right] & \text { if } \Delta L_{a} \leq 0 \\ P_{a}(t) \frac{\pi D_{0}^{2}}{4} \cdot\left[3 \cos ^{2}(\gamma(t))-1\right]+k \cdot \Delta L_{a} & \text { if } \Delta L_{a}>0\end{cases}
$$

\subsection{Braid Parameter Selection}

When designing an artificial muscle actuator for a given application, it is likely that the available supply pressure is known as well as the desired length, $L_{\max }$, and maximum diameter, $D_{\max }$, of the actuator which occurs when the braid angle, $\gamma=54.7^{\circ}$. Therefore, the optimal design is the one that maximizes the performance of the actuator in terms of the range of the muscle contraction. Due to the geometric constraints of the AMA as discussed in Section 4.2, the contraction range is directly tied to another important geometric property, the range of possible braid angles throughout the stroke of the AMA. The minimum braid angle (full muscle extension) is dependent on the strand diameter, $D_{s}$, and the number of strands in the braid, $N$, and occurs when adjacent strands make contact [131]. This relationship is presented as

$$
\gamma_{\min }=\frac{1}{2} \sin ^{-1}\left(\frac{D_{s} N}{\pi D_{0}}\right)=\frac{1}{2} \sin ^{-1}\left(\frac{D_{s} N^{2}}{b}\right)
$$

Recalling Fig. 4.3 which shows a plot of the relative force capacity of the artificial muscle actuator versus the braid angle of the mesh, it is possible to see that the force goes to zero when the braid angle reaches $54.7^{\circ}$ as previously mentioned. The upper limit of the force, which is dictated by the minimum achievable braid angle, is shown by the left vertical line. This line can shift left or right based upon the characteristics of the mesh, $D_{s}$ and $N$.

Therefore, it is necessary to consider all possible combinations of strand diameter, $D_{s}$, and number, $N$, in order to optimally design the AMA. This can be done by 
minimizing the braid angle, $\gamma_{\text {min }}$. Additionally, the stress within each individual strand of the braided mesh must also not exceed the limits of the mesh material and thus acts as a constraint on the determination of $\gamma_{\text {min }}$. The tensile stress within an individual strand within the braid is presented in the literature [131] as

$$
\sigma(t)=\frac{P_{a}(t) D(t) L(t)}{2 n N A_{\text {strand }}}=\frac{2 P D_{0}^{2} \sin ^{2} \gamma(t) \cos \gamma(t)}{N D_{s}^{2}}
$$

which is formulated by analyzing the fractional component of the hoop stress realized within an individual strand of the braided mesh. Each of the $N$ strands encircles the bladder $n$ times. This result can then be compared against the tensile limit of the mesh material, which is typically either a nylon polymer $\left(\sigma_{Y} \approx\right.$ $50 \mathrm{MPa})$ or stainless steel $\left(\sigma_{Y} \approx 500 \mathrm{MPa}\right)$. Looking at Eqs. 4.12 and 4.13 , it is seen that the effect of increasing the strand number, $N$, is that the minimum braid angle, $\gamma_{\min }$ increases (smaller contraction range) and the stress within each strand is reduced.

\subsubsection{Finite Element Analysis of Internal Bladder}

The use of the artificial muscle actuator as a means of power input to the system carries with it several advantages previously discussed. However, it is also necessary to understand the failure limits for each component. An expression for determining stress in the braided mesh was discussed in the previous section; however, it is necessary to understand the failure limits of the internal bladder due to applied pressure as well as the bladder is likely to be the weaker of the two components that make up the actuator. The likely mode of failure for the bladder is by the wall expanding through the gaps between strands, as shown in Fig. 4.4. of the braided mesh and rupturing [131]. Further, it is desirable to avoid exceeding the elastic limit of the bladder material, which varies based on material composition and strain rate, estimated as $3 \mathrm{MPa}$ for general latex rubber 
[132]. Towards this end, a finite element analysis of stress within the bladder was performed for a variety of possible combinations of actuator design parameters. The relevant parameters of each diamond shaped unit segment of the bladder are then the edge length, $E L$, and the braid angle, $\gamma$, as shown in Fig. 4.5. The edge length is a constant measure of the mesh density and is determined in the manufacturing by setting the distance between subsequent strands. The value for the edge length can be calculated as

$$
E L=\frac{b}{n N}=\frac{\pi D_{0}}{N}
$$

where $b, n$, and $N$ are the strand length, number of turns per strand, and strand number, respectively, as before. The braid angle is a measure of AMA contraction and thus changes as the AMA is pressurized. This relationship is described in Section 4.2. In addition to the parameters $E L$ and $\gamma$, it is also necessary to consider the wall thickness, $t$, of the bladder material when determining a proper design to prevent failure. Further, the pressure within the bladder, $P_{a}$ is an important consideration for evaluating the stress within the bladder wall. All of these possible variations make determination of the failure criteria for the internal bladder of the artificial muscle a difficult problem to evaluate. The complexity of this problem is further increased due to the intricacies of shell mechanics for an irregular shape. To simplify the analysis, the bladder was first divided into the smallest repeated unit which takes the form of a diamond where the sides are defined by the strands of the braided mesh, as shown in Fig. 4.5. The model used here assumes that the edges of the bladder segment are fixed in place by the braid strands. This assumption is reasonable as each side of a given segment is shared by adjacent segments which are each subject to the same conditions. Further, this model does not account for any stress relief that may be present due to the diameter of the strands restraining the bladder. This results in the overestimation of the stress within the bladder and thus means that the selection of parameters based on this model will be conservative. 


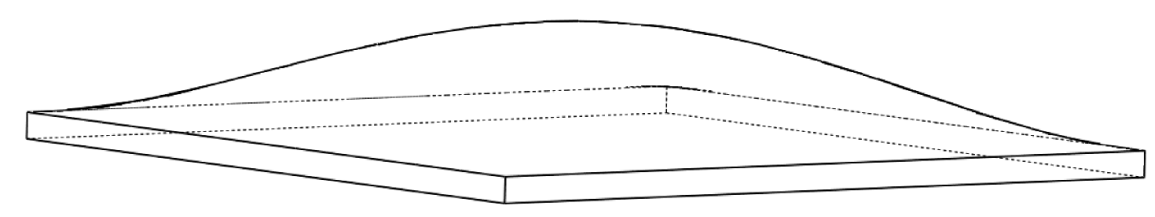

Figure 4.4: Illustration of the bladder mesh bulging through a gap in the strands of the braided sheath.

A model of this individual unit was developed in the finite element analysis software package, Abaqus Unified FEA (Dassault Systèmes). As it is possible that each of these four parameters will vary independently, it is necessary to evaluate all possible combinations. To accomplish this, the input file was scripted using the Python programming language to allow for automation of the job submission process. This script is shown in Appendix C. Briefly, the script was set up to evaluate for six possible edge lengths $(\in[0.25 \mathrm{~mm}, 1.5 \mathrm{~mm}])$, six braid angles $\left(\in\left[5^{\circ}, 55^{\circ}\right]\right)$, five bladder thicknesses $(\in[0.05 \mathrm{~mm}, 0.25 \mathrm{~mm}])$, and five internal pressures $(\in[0.25 \mathrm{MPa}, 1.5 \mathrm{MPa}])$. This results in a total of 900 possible combinations of $E L, \gamma, t$, and $P_{a}$. The range for each parameter was selected to cover the likely range encountered by the AMA when used for this application. For each combination, the script defines the geometry of the bladder segment, applies the boundary conditions and pressure load, and submits the job to the solver.

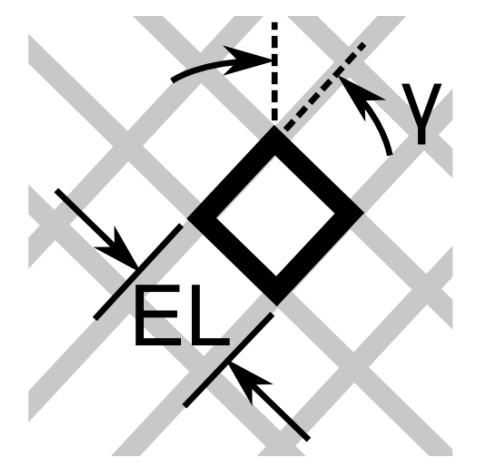

Figure 4.5: Graphic showing relevant planar dimensions describing an individual bladder segment. 
An example of the results output by the Abaqus simulation is shown in Figs. 4.6 and 4.7 where the input parameters were set to $\left[E L, \gamma, t, P_{a}\right]=\left[1.0 \mathrm{~mm}, 15^{\circ}\right.$, $0.15 \mathrm{~mm}, 700 \mathrm{kPa}$. Figure 4.6 shows the deformation of a bladder segment for a given set of parameters. As expected, the greatest deformation occurs at the center of the segment while the deformation at the edges is zero as specified by the boundary conditions. Here it is shown that the maximum calculated deformation is on the order of $0.5 \mathrm{~mm}$ for this example. Figure 4.7 shows the magnitude of the Von Mises stress within the bladder segment for the example set of parameters. Here it is shown that the highest stress concentration is calculated at the edge of the segment near the obtuse angle. The maximum stress at this location is on the order of 2.8 MPa. Moving away from the boundary, the next highest stress occurs at the same location as the maximum deformation within the segment (geometric center) and is on the order of 2.0 MPa. 


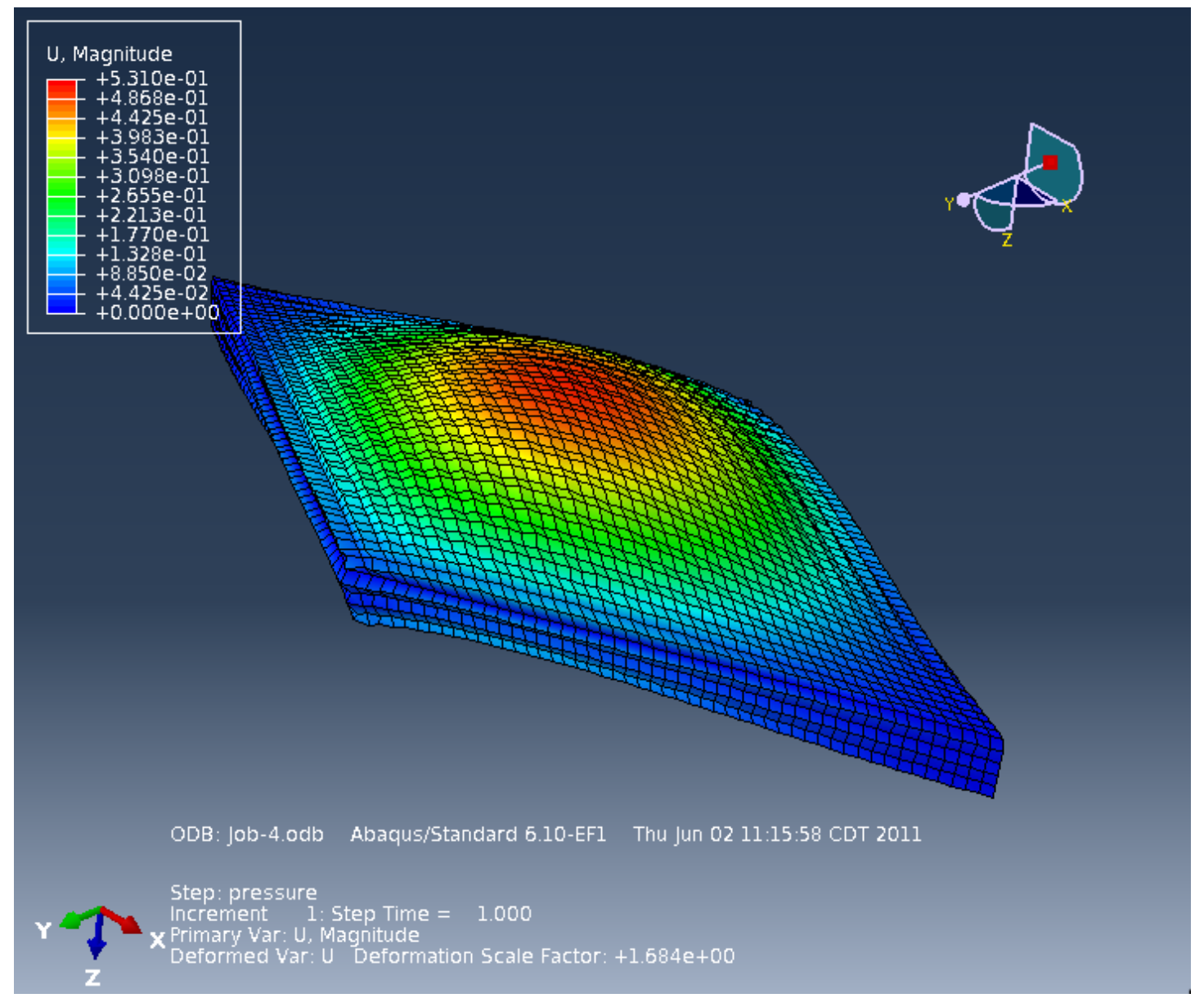

Figure 4.6: Example output of Abaqus simulation results for deformation of an internal bladder section.

The results from each of the 900 simulations (each combination of $E L, \gamma, t$, and $P_{a}$ ) were then stored in individual output files. The result of interest in this case is the maximum stress that occurs in the bladder segment. To extract this information from each of the results files, a post processing script was also written in Python (see Appendix C) to read the Von Mises stress data for each element in the mesh and then find the maximum [133]. This maximum Von Mises stress was then written to a text file along with the corresponding parameter values for each of the 900 simulations [134]. This text file represents a four dimensional array 


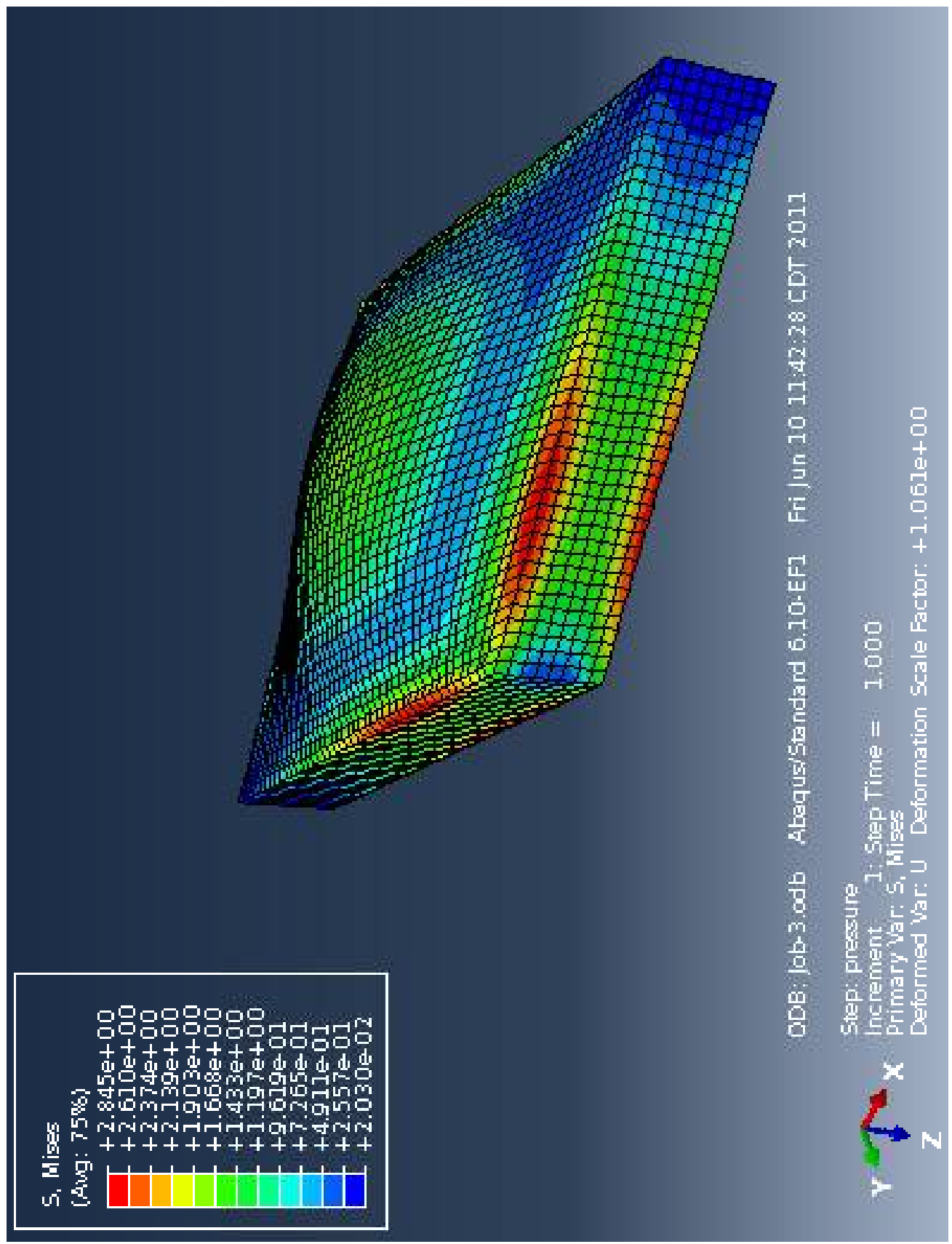

Figure 4.7: Example output of Abaqus simulation results for Von Mises stress of an internal bladder section. 
that can be used as a lookup table to find the stress corresponding to a given set of input parameters.

The array contains the values for maximum stress calculated under the given range of selected input parameters which thus necessitates the use of an interpolation to extract the relevant information for any one specific set of input conditions. For this, a cubic 4-dimensional interpolation function was used. As shown in Fig. 4.8, it was found that for any arbitrary set of input parameters where only the braid angle was varied the maximum stress occurred when the braid angle was $45^{\circ}$ for all combinations of the inputs, $P_{a}, N$, and $t$. Note that the strand number, $N$, was used in place of $E L$ for presentation of these results as it is more relevant as a design parameter. For a given value of $N, E L$ is calculated using Eq. 4.14 for submission to the interpolation function. The value for $D_{0}$ was set to $6 \mathrm{~mm}$ for this application. This result for the braid angle is true for any set of input parameters and is not unexpected since the surface area of the diamond shaped unit of the internal bladder is largest when the braid angle is $45^{\circ}$. Based on this result, it would be appropriate to use a braid angle of $45^{\circ}$ in subsequent calculations in order to account for the worst case scenario.

As discussed in Section 4.3, the strand diameter, $D_{s}$, and number, $N$, are the two critical parameters for design of the braided mesh. In terms of evaluating the bladder stress, strand diameter affects only the stress concentration at the boundaries of each bladder segment (larger strand diameter equates to lower stress concentration at the boundary). Thus assuming the most conservative condition, strand diameter is not factored into the calculation of the bladder stress. Instead, strand diameter is relevant in determination of failure within the braided mesh itself. An evaluation of the bladder stress was performed with respect to the effects of the various independent parameters. The braid angle was fixed at $45^{\circ}$ (worst case) while the strand number, $N$, actuator pressure, $P_{a}$, and bladder thickness, $t$, were varied individually. This analysis is shown in Fig. 4.9. First, it is shown in Fig. 4.9 a that there is a direct linear relationship between actuator pressure and 


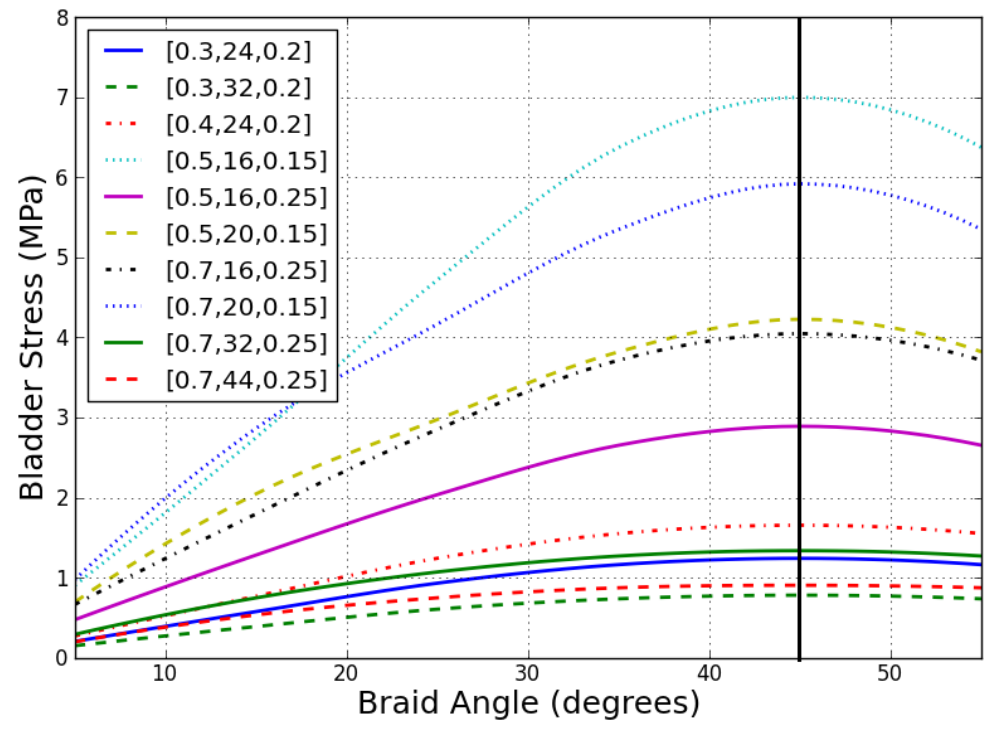

Figure 4.8: Calculation of maximum bladder stress for variable braid angle. Values indicated in the legend correspond to $\left[P_{a}(\mathrm{MPa}), N, t(\mathrm{~mm})\right]$.

stress in the bladder wall. Thus a change in actuator pressure yields a proportional change in bladder stress with a constant of proportionality that ranged from 0.65 $(N=80, t=0.25 \mathrm{~mm})$ to $31.5(N=16, t=0.1 \mathrm{~mm})$.

Next, to look at the effects of strand number and bladder thickness, the actuator pressure was set to $700 \mathrm{kPa}$ while the strand number, $N$, and the bladder thickness, $t$, were varied. As shown in Fig. $4.9 \mathrm{~b}$ varying the strand number, $N$, shows that the bladder stress increases quickly as the strand number approaches 15 where the spacing between strands becomes large. Thus, for this application, the bladder stress can be used to set the lower limit for the strand number. It is desirable to approach this lower limit as fewer strands means that the performance of the actuator is better due to having a longer contraction range.

Finally, the effects of bladder wall thickness were examined. Figure 4.9c shows that the wall stress increases quickly when the wall thickness is smaller than approximately $0.15 \mathrm{~mm}$ and changes more gradually above that value. This result 


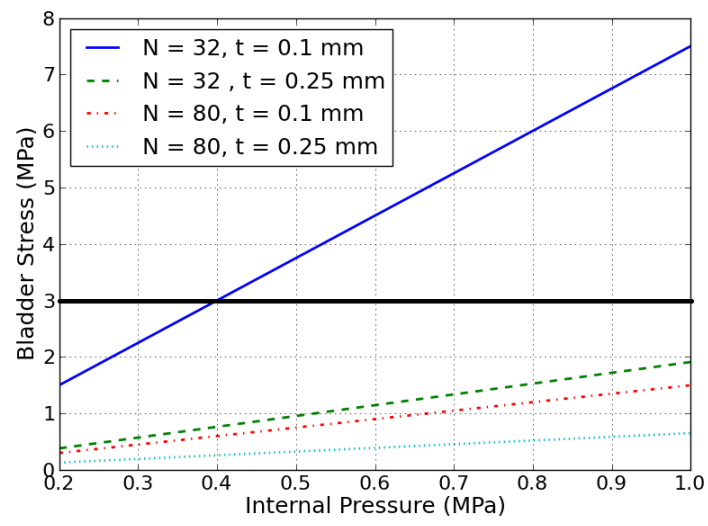

(a)

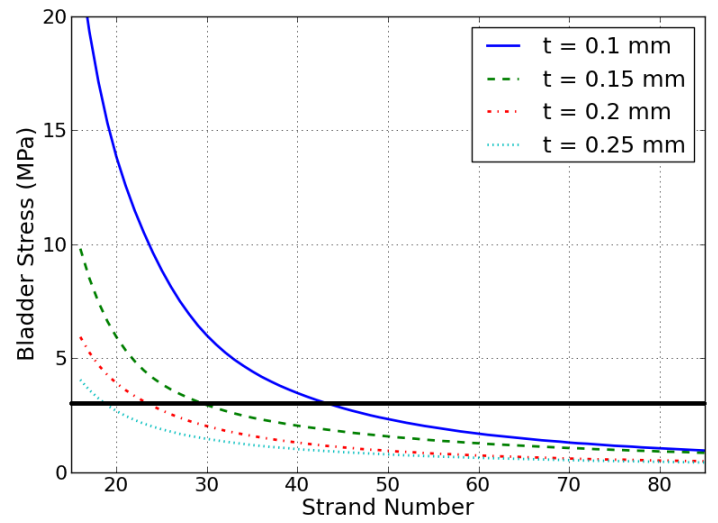

(b)

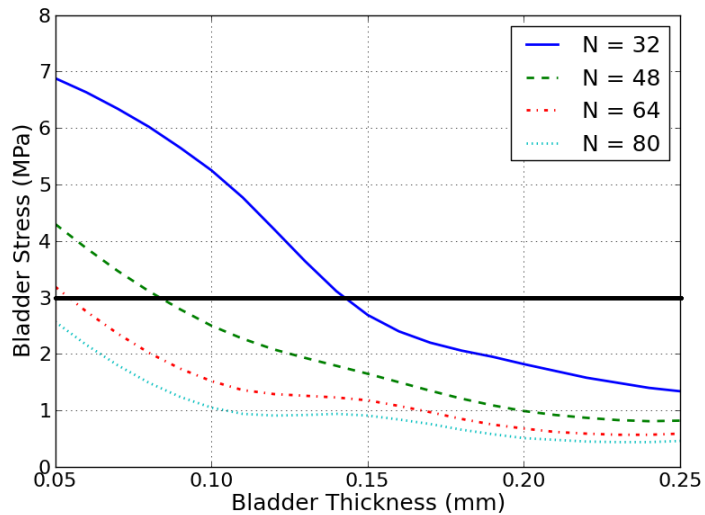

(c)

Figure 4.9: Plot of the bladder stress calculated using FEA versus (a) actuator pressure, (b) strand number, and (c) bladder wall thickness. Braid angle is set to $45^{\circ}$ for all cases. Horizontal line drawn at $3 M P a$ to indicate estimated elastic limit of a latex rubber bladder material [132]. 
is important to consider as the bladder wall thickness plays a role in determining the minimum achievable diameter of the AMA.

\subsubsection{Parameter Selection Methodology and Fabrication}

The previous text provided a means of designing the AMA by analyzing the constraints in terms of strand stress and bladder stress for a given set of input conditions. Equation 4.13, when combined with the results presented in Section 4.3.1. allows for a determination of the optimal combination of strand diameter, $D_{s}$, and strand number, $N$, within the braid for which the strand stress does not exceed the tensile strength of the braid material and the stress in the bladder wall does not exceed the limits of the bladder material. The thickness of the bladder wall is also an independent design parameter that can be minimized in order to allow the actuator to reach full elongation and therefore the wall thickness can be included as a design variable.

When designing a given muscle actuator, it is assumed that the desired maximum elongated length, $L_{\max }$, and the desired maximum diameter, $D_{\max }$, are known. From these two input parameters, the constants representing the strand length, $b$, and number of turns per strand, $n$, can be calculated using the optimized value for $\gamma_{\min }$ (Eq. 4.12) as

$$
\begin{gathered}
b=\frac{L_{\max }}{\cos \gamma_{\min }} \\
n=\frac{b}{\pi D_{0}}=\frac{b \sin 54.7^{\circ}}{\pi D_{\max }}
\end{gathered}
$$

For the braiding machines that manufacture this sort of braided mesh, the necessary input parameter is the pick count or the number of times the braid is repeated per unit length [135]. This input setting can be calculated from the optimized strand number, $N$, and the number of turns that each strand performs, $n$, over 
the actuator length as

$$
\text { Pick }=\frac{n N}{2 L_{c}}
$$

where the length, $L_{c}$, is the actuator length at the diameter of the core, $D_{c}$ that the mesh is being braided onto such that Eqs. 4.1 and 4.2 become

$$
\begin{gathered}
L_{c}=b \cdot \cos \gamma_{c} \\
\gamma_{c}=\sin ^{-1}\left(\frac{\pi n D_{c}}{b}\right) .
\end{gathered}
$$

Therefore, for a given combination of wire diameter, $D_{s}$, and strand number, $N$, the braiding machine can be configured using the appropriate pick per unit length setting calculated from Eq. 4.17.

\subsection{Testing of AMA Load Capacity}

An evaluation of the accuracy of the predicted beam load capacity as a function of AMA extension and pressure, as formulated in Section 4.2, was carried out. It was not possible to produce a muscle actuator at a scale appropriate for this application as an inner bladder material with the correct diameter was not found to be available. Thus a larger version of the muscle actuator, with an $8.8 \mathrm{~mm}$ maximum outer diameter, was produced (Fig. 4.10) using latex surgical tubing (OD $3.2 \mathrm{~mm}$, ID $1.6 \mathrm{~mm}$ ) as the internal bladder and nylon expandable mesh (OD $4.4 \mathrm{~mm}$, ID $3.2 \mathrm{~mm}$ ) as the outer sleeve, for which the minimum achievable braid angle was approximately $16^{\circ}$. The actuator from Fig. 4.10 was connected to a rigid support at one end and to a calibrated spring scale (OHAUS, $4 \mathrm{~kg}$ capacity) on the other end (Fig. 4.11). The internal bladder was inflated using an instrumented syringe (BARD Caliber Inflation Device) which provided a measure of the inflation pressure. 


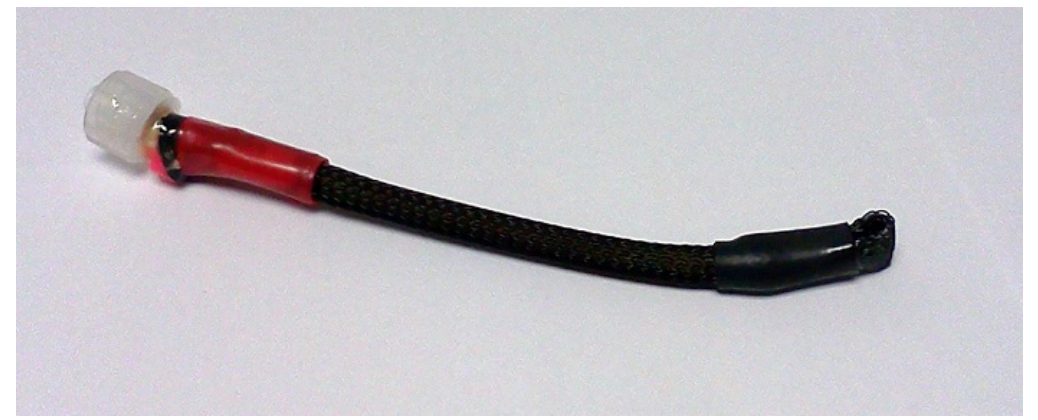

Figure 4.10: Photograph of a prototype artificial muscle actuator.

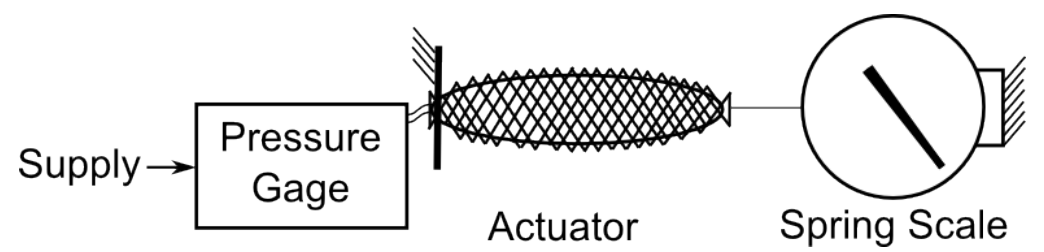

Figure 4.11: Illustration of test setup for measuring actuator load as a function of braid angle.

The procedure was performed under two static pressure conditions, $689 \mathrm{kPa}$ and $413 \mathrm{kPa}$. The pressure within the bladder was increased to the static set point and held constant while the load on the actuator was increased. For each data point, the contraction force exerted by the actuator on the spring scale was measured as well as the length of the actuator and corresponding braid angle. These results are shown in Fig. 4.12 where the solid line represents the predicted contraction force calculated using Eq. 4.8 and the dots represent the experimental data. The results show reasonable agreement, $R^{2}$ values of 0.9578 for the $689 \mathrm{kPa}$ case and 0.9079 for the $413 \mathrm{kPa}$ case, between the predicted and experimental forces. Better agreement was seen for the high-force, elongated actuator condition and greater discrepancy for the low-force, contracted muscle condition with the largest error occurring at a braid angle of $50^{\circ}$ where the error is $50 \%$ of the predicted value (689 $\mathrm{kPa}$ case). These finding are similar to results reported in the literature [129, 131]. Deviations between the experimental and theoretical actuator forces are likely due to frictional effects which become more observable at higher braid 
angles.

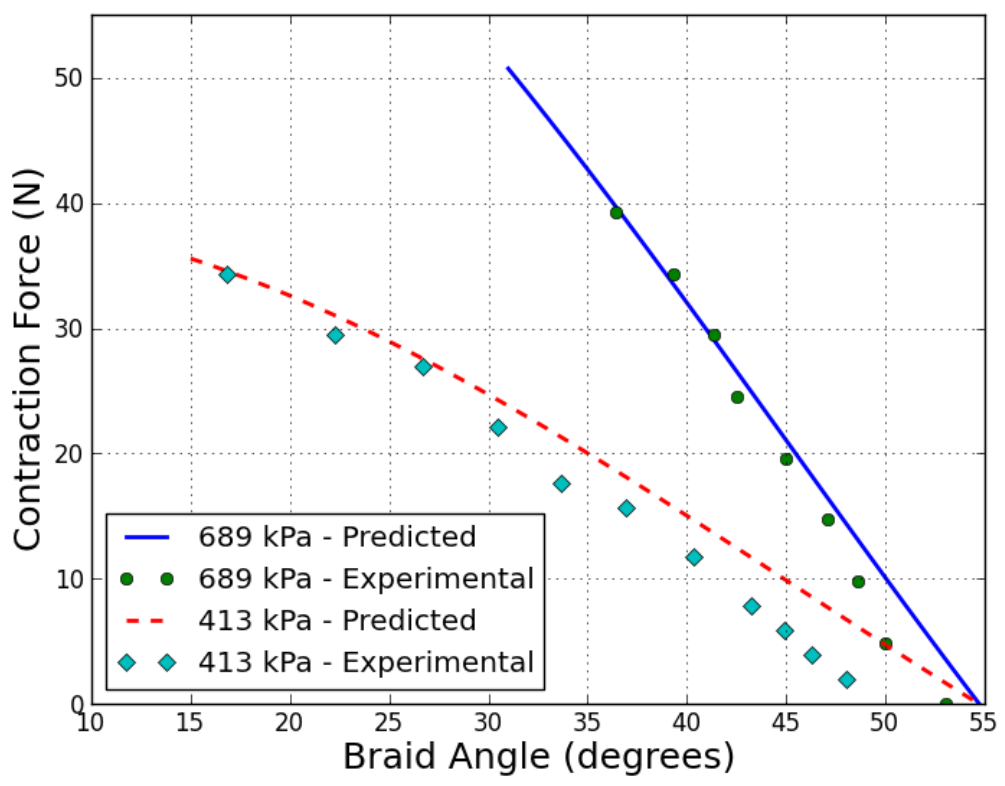

Figure 4.12: Plot of force output from the artificial muscle actuator predicted analytically (solid line) and determined experimentally (dots).

\subsection{Summary of Actuation Unit Design}

The use of artificial muscle actuators for robotic applications has been expanding as they provide several advantages including dexterous mobility and compliance when coming into contact with other surfaces or objects [136]. This is particularly important in the application of minimally invasive surgery where the robot maneuvers in an unpredictable and sensitive environment. Further, the use of a hydraulic artificial muscle actuator for this purpose provides the opportunity for greater force output for the given size constraints, as shown in Fig. 4.3 where it is seen that the theoretical load capacity of the AMA is up to three times that of a conventional hydraulic actuator for the same diameter. Noting, however, that this advantage is lower in practice based on the real value for the minimum achievable 
braid angle. The real force capacity advantage is likely in the range of 2.5 to 2.85 times the traditional hydraulic actuator based on an achievable minimum braid angle of between $20^{\circ}$ and $10^{\circ}$. In Section 4.2, a method for modeling the AMA and its load capacity was given showing that actuator force is a function of internal pressure and actuator length. The procedure for defining this method includes several simplifying assumptions such as the absence of considerations for frictional effects; however, it was demonstrated that at the prototype scale for this application, the predicted output compared well with the experimental results. If the actuators were to be decreased in size by increasing the operating pressure, the effects of these errors would become more pronounced [128]. The determination of these errors requires experimental analysis of appropriate small scale AMAs. As was shown, the maximum contraction of the AMA is set by the braid geometry at a braid angle of $54.7^{\circ}$. However, the maximum elongation of the AMA is something that can be designed and thus allows for an optimization of the AMA characteristics in order to achieve the greatest stroke length while avoiding failure.

The methods presented here make it possible to identify the appropriate braid characteristics to achieve maximum AMA performance. Maximum performance is obtained by minimizing the achievable braid angle. The minimum braid angle is dependent on the number of strands within the braided mesh and the diameter of those strands [131]. The limiting conditions placed on these two quantities are the yield stress of the strands and the stress limit of the inner bladder. The yield stress of the strand can be calculated directly knowing the strand number, strand diameter, pressure, and braid angle of the AMA (see Eq. 4.13). The stress within the bladder requires more careful consideration as it is also parameter dependent but is less well defined in terms of a solution method. Therefore, to determine bladder stress, a finite element analysis was used. Here it was shown that under all tested conditions the maximum bladder stress occurred at a braid angle of $45^{\circ}$. Thus in performing an optimization calculation, the braid angle can be set at this 
value to eliminate one of the variables. Further, since it is desired to minimize the achievable braid angle, the bladder wall thickness can be set at a low value for this calculation as well while having the option of increasing the thickness to permit higher operating pressures. Finally, under the appropriate stress constraints, it was shown that it is possible to calculate the smallest allowable strand diameter and the smallest number of strands that can be used to produce a usable AMA.

Using a prototype AMA, it was demonstrated that a load capacity of $40 \mathrm{~N}$ was possible with a bladder pressure of $689 \mathrm{kPa}$. However, this value does not represent the maximum achievable value since the muscle was not in its fully extended condition when the limit of the spring scale was reached. The theoretical prediction was found to be accurate at the tested braid angles with the exception of observed deviations as the braid angle approached its maximum limit. If the theoretical calculation for AMA load capacity is extended towards smaller braid angles, then the load capacity of the prototype AMA would approach $80 \mathrm{~N}$ as the braid angle approached $10^{\circ}$. If we then extend this model to the design scale with a maximum outer actuator diameter, $D_{0}=6 \mathrm{~mm}$, the predicted load capacity of the AMA would be $37.2 \mathrm{~N}$ as the braid angle approached $10^{\circ}$ for the same supply pressure. 


\section{Chapter 5}

\section{Control Valve}

This chapter describes the design and testing of a novel hydraulic control valve for use in the minimally invasive surgical manipulator. Section 5.1 provides motivation for the development of this design followed by a description of the various considerations relevant to the valve and its application, such as the method of manipulation for the valve. In Sections 5.2 and 5.3 a theoretical valve design is presented as well as a mathematical description of the operating principles. Next, Section 5.4 discusses two possible methods of valve activation, piezoelectric and electromagnetic. In Section 5.5, two physical realizations of the valve design are presented as well as a discussion of how they operate relative to the theoretical design. Finally, Section 5.6 presents the results of experimental testing performed on valve prototypes which are used to evaluate various design options and help inform the selection of the final configuration. 


\subsection{Motivation and Related Work}

The ability to control the flow of the fluid is essential to any hydraulic system. Typically this is achieved through the use of valves which can control whether the flow is ON or OFF and possibly the rate at which the fluid is flowing. The method by which flow is stopped can include rotational motion such as in a rotary valve, linear motion such as in spool or poppet valves, or with a flapper valve that acts parallel to the flow direction. Additionally, proportional control can be achieved through varying the size of the valve opening or by control of an ON/OFF valve. Either method carries advantages and disadvantages. A proportional valve may provide more straight forward control methods; however, it introduces additional difficulties such as power consumption which can be particularly troublesome for small scale applications. Alternatively, an ON/OFF valve may have a simpler design but considerations must be made for inertial and transitional losses. Another possible solution is the use of digital hydraulics which enables a near analog output using digital or ON/OFF components [137]. For all methods, precise flow control is an integral component of a successful hydraulic system.

There has been a significant amount of research committed to the design and evaluation of flow control valves. However, the area particularly relevant for the present application is valve miniaturization due to the limited space availability within the surgical tool. As previously mentioned, the field of fluid power at the micro-scale is referred to as microfluidics, a field with origins beginning in the 1990s and has quickly expanded to suit a variety of potential applications [78]. Much of the research in microfluidic valves makes use of any one of a short list of enabling technologies including electrostatic, magnetic, piezoelectric, thermal, chemical, or pneumatic methods [138, 139, 140, 141, 142, 143]. Of these options, possible valve operation methods such as piezoelectric or electrostatic can provide interesting solutions to the problem of flow control and have been explored extensively for control of pneumatic systems [144, 145]. A MEMS based design solution 
was explored as a way to achieve flow control for this application. However, several difficulties were encountered in terms of valve robustness and fabrication. A MEMS based design is presented in Appendix $\mathrm{A}$ as a possible design alternative for future exploration.

As previously discussed, the availability of a valve for this application which meets the needs of the device in terms of controllability and size was not found to be available commercially. However, there are several approaches being pursued academically to bridge the gap between traditional- and micro-fluidics. Two methods presented in the literature include the use of magnetics for valve actuation [146, 147]. Fu et al. present a solution designed to use $3 \mathrm{~mm}$ diameter iron balls to facilitate closure of the valve orifice [146]. The position of the ball is driven by a coil with a length of $10 \mathrm{~mm}$ and a diameter of $8 \mathrm{~mm}$. The valve was demonstrated to operate with supply pressures of $50 \mathrm{kPa}$ to $200 \mathrm{kPa}$ and could operate in proportional mode up to $112.5 \mathrm{kPa}$ before the ball was pulled into the valve seat. This design is promising but has too large of a footprint and operates at too low of pressures for use in this application. Another solution presented by Pernod et al. has a similar pressure capacity and is operated by a $1 \mathrm{~mm}$ long by 3 mm diameter permanent magnet combined with a 500 winding coil to manipulate the valve membrane [147]. This valve has a smaller footprint than the design presented by $\mathrm{Fu}$ et al. but does not yet meet the requirements for this application in terms of size and controllability. Finally, Peirs et al. investigated the use of both electromagnetic and piezoelectric methods for valve manipulation [80]. In that study it was found that either approach provided significant challenges for producing the desired performance safely and consistently. In particular they encountered challenges with integrating the valves into their design under similar size constraints to the present application. Therefore, given the lack of available solutions either commercially or academically, the design of a new valve to fit this application and meet the necessary design considerations was undertaken. 


\subsection{Control Valve Design}

In order to achieve precise manipulation, it is necessary to obtain a control valve that will provide a controllable actuator pressure while occupying as little space as possible within the device. A review of the available commercial valves and common micro-fluidic valve solutions was conducted and no existing valve design was found suitable for the present application due to the limitations of high supply pressure, proportional control, and method of activation. Therefore, development of a novel control valve with a diameter of $6 \mathrm{~mm}$, capable of manipulating high pressure flows, which could be on the order of 0.7 to $7 \mathrm{MPa}$ for hydraulics, on a small scale was performed.

For this application, the outlet of the valve will be connected to the AMA described in the previous chapter. To provide useful manipulation, it is the feed pressure, $P_{a}$, to the actuator that must be regulated by the valve such that the contractile force produced by the actuator (Eq. 4.8), which is proportional to $P_{a}$, can be manipulated. Each actuator provides one directional contraction only and thus an arrangement of three actuators at a minimum operated differentially and antagonistically such that the manipulator can operate in three-dimensional space (discussed in greater detail in Section 6.2.2). For each of the actuators, a simple hydraulic circuit such as is shown in Fig. 5.1 can be used. This circuit consists of an upstream, high-pressure supply, $P_{s}$, and a fixed orifice flow restriction, $R_{1}$, as well as a downstream variable orifice flow restriction, $R_{2}(t)$, and a return to atmosphere, $P_{\text {atm }}$. Between the two flow restrictions is the feed point for the actuator, $P_{a}(t)$. By adjusting the downstream restriction relative to the fixed, upstream restriction it would then be possible to manipulate the pressure at the actuator feed point as a pressure divider. For this purpose, a flapper-style valve can be used as a variable orifice [148]. With appropriately sized orifices, the flapper would then act as the input for manipulating the downstream orifice within its available stroke range. 


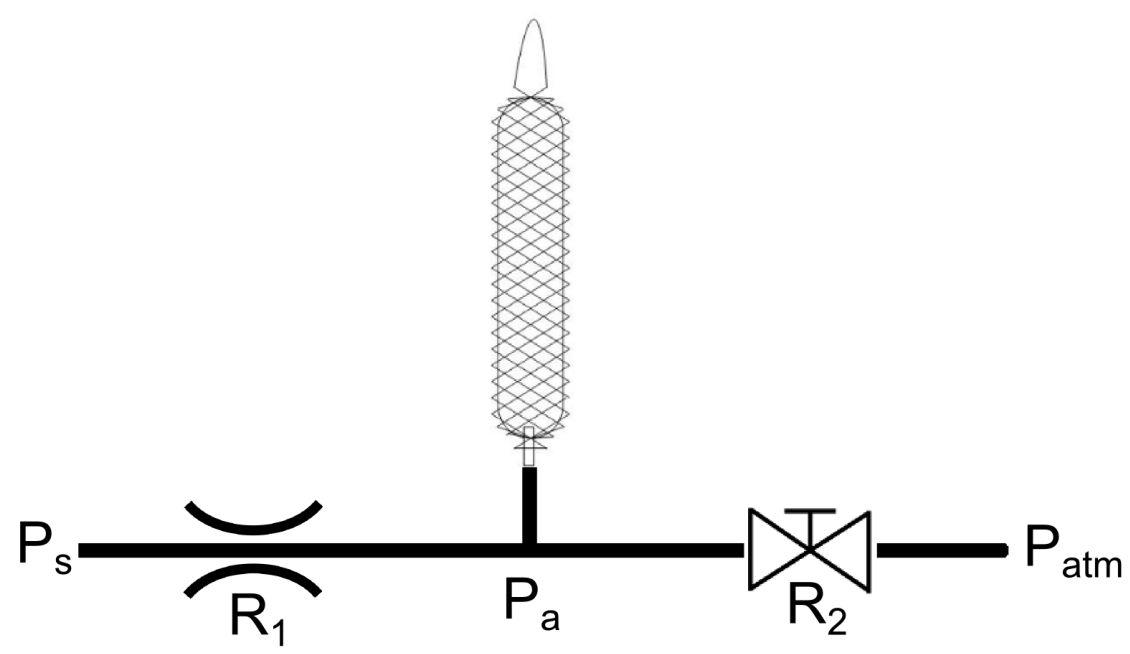

Figure 5.1: Hydraulic circuit concept for control of a single actuator.

As each manipulator segment would require three actuators, the hydraulic circuit could be modified to the form shown in Fig. 55.2, which shows three sets of upstream orifices, three actuator feed points, and a single central flapper that is used to regulate the three actuators in a differential/antagonistic fashion as required. As such, when the flapper moves nearer to one of the downstream exit orifices (increasing the pressure at the associated actuator feed point), it is moving away from the other two exit orifices at the same time (decreasing the pressure at the associated actuator feed points).

This style of valve has the advantages of low complexity and insensitivity to contaminants in the fluid [148. Further, the manipulation of the valve can be intuitively mapped to the output of the manipulator since each have three inputs and are operated differentially/antagonistically. One of the basic principles of a flapper style valve is that there is a constant leakage flow. Thus the supply must be capable of accomodating the leakage. This presents little concern as the flow rate required to inflate the actuators is significantly smaller than the available flow rate of the supply, five liters per hour based on actuator fill time (assuming 


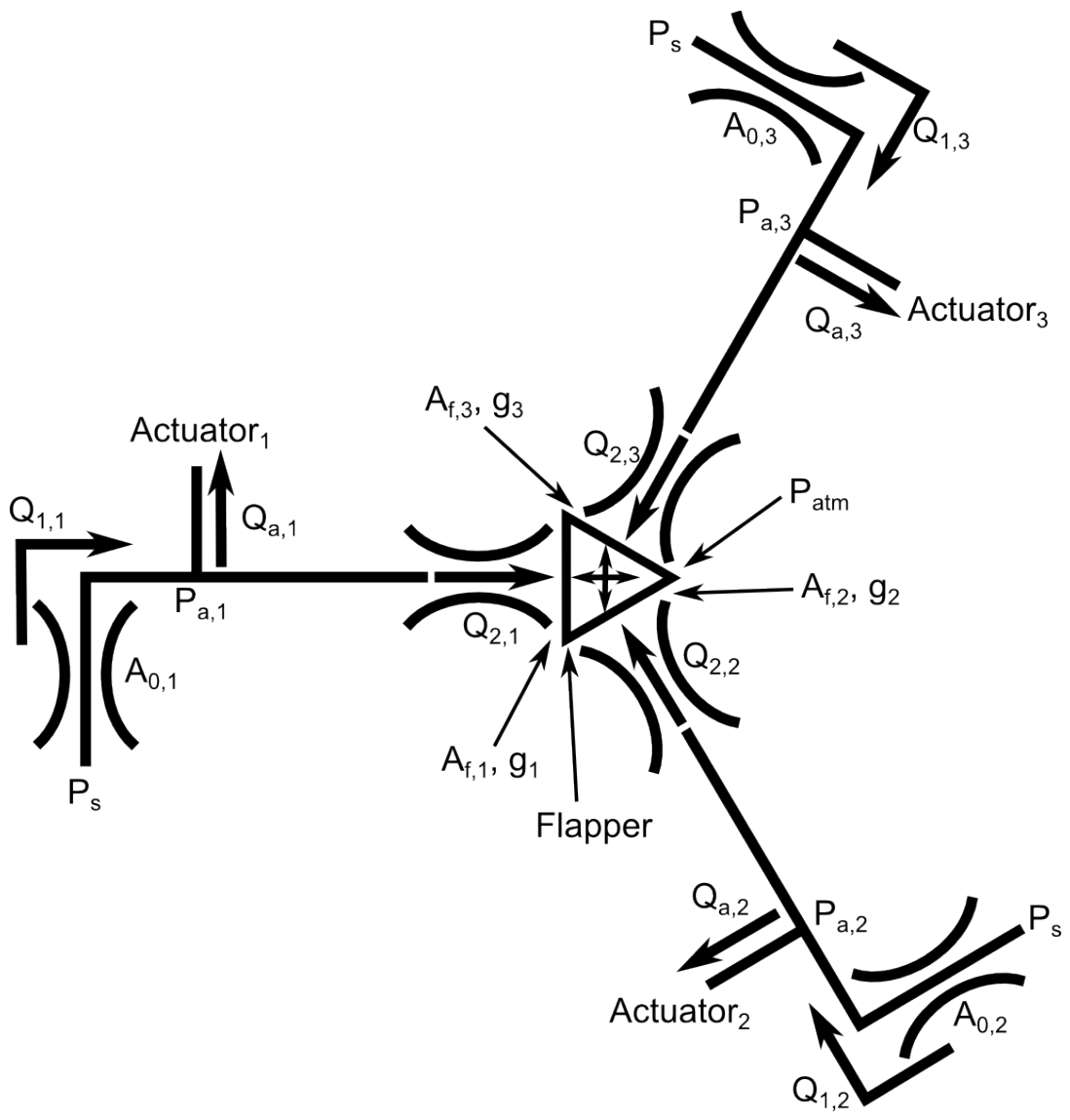

Figure 5.2: Three actuator hydraulic circuit concept for the flapper style valve design.

$689 \mathrm{kPa}$ supply pressure, 0.5 seconds fill time for a single actuator) for a design scale actuator versus 55 liters per hour based on available flow rate for endoscopic irrigation pumps [148, 149]. Further, the capabilities of existing irrigation pumps do not constrain the design as the supply pump could be modified to meet the necessary requirements. 
111

\subsection{Analytical Description of Flapper Based Valve Design}

The valve design is modeled as a flapper style valve and thus the flow through each upstream orifice $\left(Q_{1, i}\right)$ and each downstream valve orifice $\left(Q_{2, i}\right)$, shown in Fig. 5.2, is calculated in Eqs. 5.1 and 5.2.

$$
\begin{gathered}
Q_{1, i}(t)=A_{0} C_{d 0}\left[\frac{2}{\rho}\left(P_{s}-P_{a, i}(t)\right)\right]^{\frac{1}{2}} \\
Q_{2, i}(t)=A_{f, i}(t) C_{d f}\left[\frac{2}{\rho} P_{a, i}(t)\right]^{\frac{1}{2}}=\pi D_{N} g_{i}(t) C_{d f}\left[\frac{2}{\rho} P_{a, i}(t)\right]^{\frac{1}{2}}
\end{gathered}
$$

where $P_{s}$ is the supply pressure and $P_{a, i}$ is the pressure at each actuator feed point. Here it is shown that the upstream flow rate is consistent with the standard orifice equation, where $A_{0}$ and $C_{d 0}$ are the area and discharge coefficient of the upstream orifice, respectively, while the downstream flow exiting the downstream orifice is restricted by the flapper. In this case the curtain area $\left(A_{f, i}(t)\right)$ rather than the orifice area is used in the flow rate calculation. The curtain area is $\pi D_{N} g_{i}(t)$, where $D_{N}$ is the nozzle diameter and $g_{i}(t)$ is the gap between the orifice and the flapper. Further, the discharge coefficient for the flapper orifice $\left(C_{d f}\right)$ is typically on the order of $0.8 \cdot C_{d 0}$ [148]. In both of the previous equations, $\rho$ represents the density of the fluid used. The gap, $g_{i}(t)$, acts as the input variable for control of the valve and varies based on the spatial position of the flapper. If the threedimensional position of the flapper is described in Cartesian coordinates where the XY-plane describes planar motion of the flapper and the Z-axis describes out-of-plane motion, then the change in the size of the gap, $g_{i}(t)$, can be found as

$$
\left[\begin{array}{c}
\Delta g_{1}(t) \\
\Delta g_{2}(t) \\
\Delta g_{3}(t)
\end{array}\right]=\left[\begin{array}{ccc}
1 & 0 & -\sin \beta \\
-\sin 30^{\circ} & \cos 30^{\circ} & -\sin \beta \\
-\sin 30^{\circ} & -\cos 30^{\circ} & -\sin \beta
\end{array}\right]\left[\begin{array}{c}
\Delta x(t) \\
\Delta y(t) \\
\Delta z(t)
\end{array}\right]
$$


assuming that the gap, $g_{1}$, is aligned with the $\mathrm{X}$-axis and $\beta$ is the taper angle of the flapper as discussed further in Section 5.5. Equation 5.3 assumes that the downstream orifices are equally spaced around the circumference of the valve and that a positive change in $g_{i}(t)$ corresponds to closure of the gap.

Additionally, the flow rate to or from the actuator must satisfy the difference between the upstream and downstream flow rates for each actuator as in Eq. 5.4.

$$
Q_{a, i}(t)=Q_{1, i}(t)-Q_{2, i}(t)
$$

Since $Q_{a, i}(t)$ is equal to the rate of change of actuator volume, $\dot{V}_{i}(t)$, under the incompressibility assumption, actuator flow rate, $Q_{a, i}(t)$, can be found with the first time derivative of Eq. 4.3, which results in

$$
Q_{a, i}(t)=\dot{V}_{i}(t)=\frac{1}{4 \pi n^{2}}\left(b^{2}-3 L_{a, i}(t)^{2}\right) \cdot \dot{L}_{a, i}(t)
$$

where the actuator length, $L_{a, i}(t)$, is dependent on beam geometry (will be described in Chapter 6). This allows for a calculation of $P_{a, i}(t)$ through a combination of Eqs. 5.1, 5.2, and 5.4 as

$$
\begin{aligned}
P_{a, i}(t) & =\left[\rho Q_{a, i}^{2}+2 A_{0}^{2} C_{d 0}^{2} P_{s}-\frac{2 \rho A_{0} C_{d 0} Q_{a, i}}{A_{0}^{2} C_{d 0}^{2}+A_{f, i}^{2} C_{d f}^{2}}\left(A_{0} C_{d 0} Q_{a, i}\right.\right. \\
& \left.\left.+A_{f, i} C_{d f} \sqrt{\frac{2 P_{s} A_{0}^{2} C_{d 0}^{2}+2 P_{s} A_{f, i}^{2} C_{d f}^{2}-\rho Q_{a, i}^{2}}{\rho}}\right)\right]\left(2 A_{0}^{2} C_{d 0}^{2}+2 A_{f, i}^{2} C_{d f}^{2}\right)^{-1}
\end{aligned}
$$

It is convenient to establish the working range of the control pressure by examining the blocked-load condition, $Q_{a, i}(t)=0$. In this steady-state condition when actuator volume is constant, the upstream and downstream flow rates become 
equal and the equations simplify to

$$
\frac{P_{a, i}}{P_{s}}=\left[1+\left(\frac{C_{d f} A_{f, i}(t)}{C_{d 0} A_{0}}\right)^{2}\right]^{-1}
$$

This relationship is shown in Fig. 5.3 [148]. This plot shows that there is a large region of approximately linear response between 0.4 and 1.6 for the orifice ratio. Further, it can be seen that the pressure response flattens out at an orifice ratio of approximately 3.0, meaning that this is a reasonable upper limit placed on the size of the flapper valve exit area relative to the upstream flow restriction.

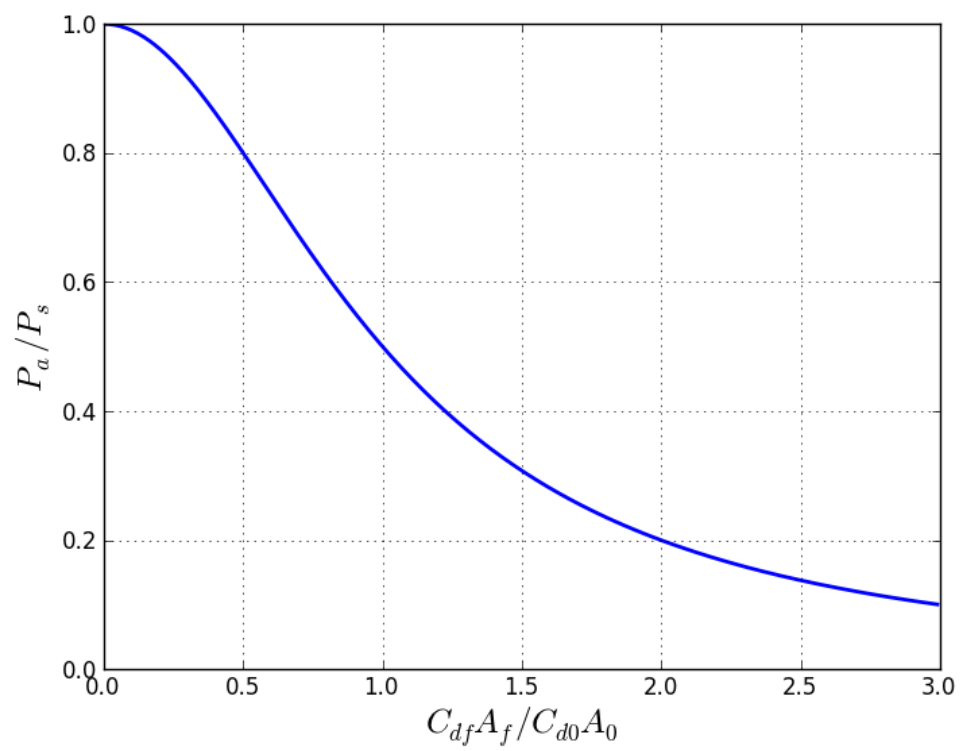

Figure 5.3: Plot of pressure ratio versus orifice ratio for a flapper valve.

\subsection{Valve Actuation}

Under the conditions described in the previous section, it is necessary to consider the options available to provide operation of the valve. The two most promising 
solutions are piezoelectric and electromagnetic [144, 145]. Each of these valve actuation methods must be able to fit within the constraints of the device and the available space within the valve, manipulate the three downstream orifices either with one component or three single-acting components, and provide analog manipulation over the range of motion specified in Section 5.3. Discussion of the piezoelectric and electromagnetic options is provided here with recommendations of the most viable solution for this application.

\subsubsection{Piezoelectric Actuation}

One possible option for valve actuation is to use a piezoelectric (PZ) actuator as the input method for the valve, which has the advantage of being MRI compatible. Piezoelectric actuators have characteristics of high force output and fast response time. While large forces are possible with PZ actuators, large displacements are difficult to produce, typically in the range of $0.1 \%$ strain as a maximum [150]. To overcome the strain limitations it may be necessary to consider methods of force amplification. One such mechanism has been developed with a capability of increasing the strain output to $1.4 \%$ using a single amplification or $20 \%$ strain when using a double mechanism [151. This type of strain amplification comes at the cost of force output and overall size of the actuator. An example comparison of the use of a PZ actuator for valve manipulation is shown in Table 5.1. Here it is assumed that the force output of a PZ stack is $30 \frac{\mathrm{N}}{\mathrm{mm}^{2}}$ and the strain without amplification is $0.1 \%$. These numbers relay the fact that when an amplification layer is added to the PZ stack, the force requirement increases and thus the crosssectional area $(\mathrm{W} \cdot \mathrm{H})$ of the stack must get larger. It is evident from these dimensions that for the application to the previously presented valve, it would be necessary to give significant consideration to the size of the PZ stack. Another approach to this would be to use a bi-morph style PZ actuator. However, similar size limitations are present and must be overcome. 
Table 5.1: Comparison of PZ stack with and without amplification.

\begin{tabular}{lc}
\hline Actuator Type & Stack Dimensions $(\mathrm{L} \times \mathrm{W} \times \mathrm{H})$ in $\mathrm{mm}$ \\
\hline No Amplification & $30 \times 0.4 \times 0.4$ \\
Single Layer & $2.14 \times 1.54 \times 1.54$ \\
Two Layer & $0.15 \times 5.8 \times 5.8$ \\
\hline
\end{tabular}

Some work exploring the use of PZ actuators for valve control has been presented in the literature where it was suggested that a PZ stack can be used effectively for this application but care must be taken to limit the effects of moisture [80]. Exposure to excessive humidity was found to limit the life of the actuator. Other limitations of a PZ based design that must be taken into account include the driving voltage and operating frequency. Piezoelectric devices require high voltage to operate; typically in the range of 100 volts or greater. This would present considerable risk to the patient and thus care would need to be taken to mitigate this risk. Finally, piezoelectric devices typically exhibit poor performance at low operating frequencies due to charge drain within the device which results in diminished actuator performance [152]. Manipulation of the valve would have to account for this which may limit the usage scenarios of the valve under proportional control.

\subsubsection{Electromagnetic Actuation}

Electromagnetic actuation is another option for manipulation of the valve discussed in Section 5.2. The use of this type of actuation has the disadvantage of not being MRI compatible but has the advantages of being low complexity, inexpensive, and able to operate in an aqueous environment. The basic realization of an electromagnetic actuator is such that a magnetic field is generated by wound coils of small diameter magnet wire. An example of a coil produced using 44 AWG (American Wire Gauge) wire which has a diameter of $0.05 \mathrm{~mm}$ is shown in Fig. 5.4. The key design parameter for the driving coil is the wire diameter which is 
available commercially in a variety of discrete sizes. The resistance of the wire is then directly a function of the wire diameter and length necessary to produce the coil. Further, the current applied to the wire is variable such that the coil produces the desired electromagnetic force to manipulate the valve. The driving coil has to be capable of closing the gap between the coil and the flapper against the force of the saline exiting the orifice. The force that the solenoid is capable of producing can be calculated by first finding the magnetic flux density, $B$, from Ampere's Law [153] as

$$
B=\frac{N I \mu}{g}
$$

where $I$ is the coil current, $N$ is the number of coil turns, $g$ is the gap between the solenoid and the flapper, and $\mu$ is magnetic permeability of the core material. Force generated can then be expressed as

$$
F=\frac{B^{2} A}{2 \mu}=\frac{(N I)^{2} \mu A}{2 g^{2}}
$$

where $A$ is the cross sectional area of the solenoid. By rearranging Eq. 5.9 the current requirement for closure of an electromagnetic relay can be shown as

$$
I=\sqrt{\frac{2 g^{2} F}{N^{2} \mu A_{N}}}=\frac{g}{N} \sqrt{\frac{2 F}{\mu A}} .
$$

It is apparent that the closing force, $F \propto I^{2} N^{2}$. Further, it is necessary to consider that the possible number of coil turns, $N \propto \frac{1}{D_{w}^{2}}$ based on circle packing density [154] and $I \propto D_{w}^{2}$ for a given force requirement based on wire resistance being inversely proportional to cross-sectional area, where $D_{w}$ is the wire diameter. Putting these relationships together demonstrates that the closing force capacity of a given coil is independent of wire diameter. Another consideration is the power consumed by the coil during operation. Knowing that the power carried by the wire is related to current and resistance as $P=I^{2} R$, it is seen that $P \propto D_{w}^{2}$ as well since wire resistance, $R \propto \frac{1}{D_{w}^{2}}$. This implies that for larger wire 
diameter, more power is introduced to the system and thus more heat has to be dissipated from the driving coil in order to prevent a short by melting the wire insulation. This suggests that the smallest possible wire would be the most ideal for the coil in terms of heat dissipation from the system. One additional benefit to selecting the smallest possible diameter for the coil wire is that as the diameter decreases, the achievable packing density for a given available space increases [154]. By optimizing the packing density, it is possible to achieve the necessary force capacity from the solenoid for a smaller outer diameter. For problems of this sort, it is found that the packing density converges towards a volumetric efficiency of $90.69 \%$ as the wire diameter decreases [155]. For this application, greater than $90 \%$ efficiency is achieved for diameters smaller than $0.21 \mathrm{~mm}$, based on the analysis of Peikert et al., 1992 [154]. Therefore, any wire diameter smaller than this limit provides reasonable packing density. Given this analysis and the available sizes of magnet wire, wire with a diameter of $0.05 \mathrm{~mm}$ was selected for prototyping and testing purposes.

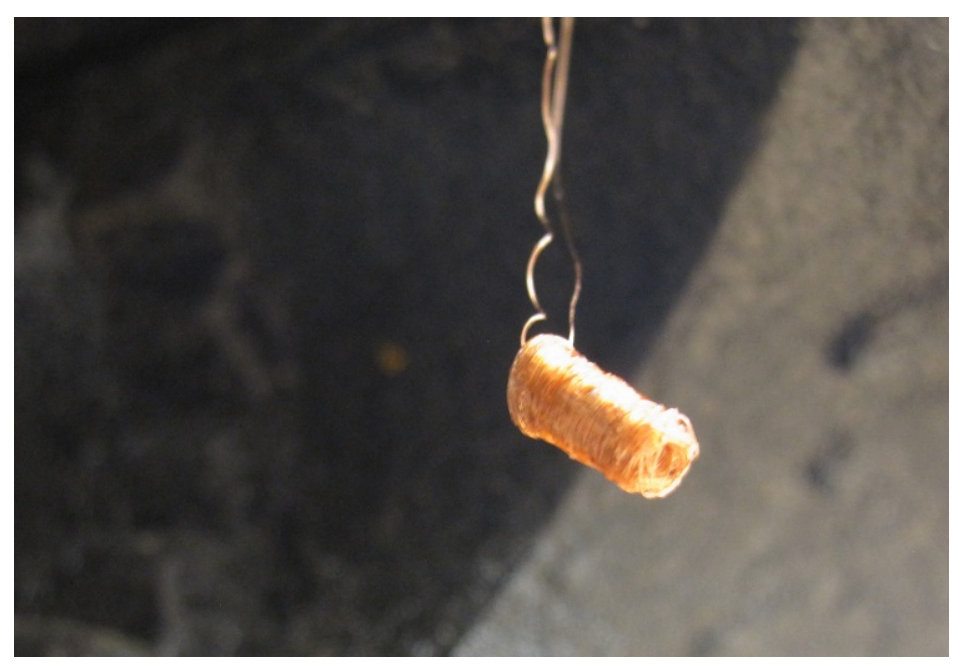

Figure 5.4: Photograph of a 44 gauge wire coil used to test heat dissipation.

Revisiting Eq. 5.10, it is possible to apply the design constraints set forth in Section 5.3

$$
\frac{C_{d f} A_{f}}{C_{d 0} A_{0}}=3
$$


and $C_{d f}=0.8 \cdot C_{d 0}$ together with the equation for the flapper gap

$$
g=\frac{A_{f}}{\pi D_{N}}=\frac{3.75 A_{0}}{\pi D_{N}}
$$

If the force requirement for the flapper is equal to the pressure drop across the valve multiplied by the nozzle area, $A_{N}$, then Eq. 5.10 becomes

$$
I=\frac{g}{N} \sqrt{\frac{2 P_{a}}{\mu}} .
$$

Finally, the number of coils of wire, $N$, that can fit within can be approximated using the optimal packing density and the available area, $L_{c} \cdot D_{c} / 2$, where $L_{c}$ and $D_{c}$ are the length and effective diameter of the coil, as

$$
N=\frac{L_{c} \cdot D_{c} \cdot 0.9069}{\frac{\pi}{4} D_{w}^{2}}
$$

Putting it all together,

$$
I=\frac{3.75 A_{0}}{\pi D_{N}} \cdot \frac{\frac{\pi}{4} D_{w}^{2}}{L_{c} \cdot D_{c} \cdot 0.9069} \cdot \sqrt{\frac{2 P_{a}}{\mu}}
$$

Substituting in the appropriate values for this design and assuming 242 turns of wire are used to form the coil around a steel core, it is found that the necessary driving current is $41.0 \mathrm{~mA}$. Therefore, based on the wire diameter previously selected, it is calculated that the power being dissipated into the system is on the order of $7.8 \mathrm{~mW}$ where the resistance of the wire is calculated using the resistivity of copper.

Since the energy entering the system through the coil is primarily dissipated through heat, it is necessary to determine how much of that heat can be delivered into the fluid that is flowing past the coil. To evaluate this, it is necessary to develop an understanding of the capacity of the fluid to remove heat from the 
coil as it passes through the valve. The heat transfer coefficient for this flow is calculated as

$$
h=\frac{k_{w}}{D_{H}} N u
$$

where $k_{w}$ is the thermal conductivity of the fluid, $D_{H}$ is the hydraulic diameter of the flow path, and $N u$ is the Nusselt number. As the flow within the valve is likely to be turbulent based upon the flow rate of the fluid and the diameter of the orifices, the Dittus-Boelter correlation [156] was used to calculate $N u$ as

$$
N u=0.023 \operatorname{Re}^{0.8} \operatorname{Pr}^{n}
$$

where $R e$ is the Reynolds number $(10,298)$ calculated on the basis of the hydraulic diameter, $\operatorname{Pr}$ is the Prandtl number (13) estimated for $0.9 \%$ water salinity [157], and $n$ is equal to 0.4 since the coil temperature is greater than the fluid temperature (heating condition) [158]. Finally, the heat flow in watts is calculated as shown in Eq. 5.18 where $A$ is the surface area of the fluid interaction and $\Delta T$ is the temperature difference between the coil and the bulk fluid.

$$
Q=h A \Delta T=\frac{k_{w}}{D_{H}} N u A \Delta T
$$

Substituting in the design parameters for this valve (Section 6.3) and the properties of saline $\left(k_{w}=578.8 \frac{\mathrm{mW}}{\mathrm{m} \cdot \mathrm{K}}[159,160]\right)$ it is found that the available heat flow into the bulk fluid is on the order of $166 \mathrm{~mW}$ per unit kelvin of temperature difference. Thus as the temperature difference increases, the heat flow into the fluid increases as well. Since the total capacity for heat flow into the fluid is significantly greater than the maximum power entering the coil, the coil temperature should stabilize below the melt temperature of the wire insulation even when operating at maximum current. 


\subsection{Description of Valve Realizations}

Two possible realizations of this valve design were explored. The first consists of three flapper nozzle valves [148] controlled by one single tetrahedral valve plug that plays the role of the flapper for each of the orifices (see Figs. 5.5). A second realization (Fig. 5.6) of the flapper style valve design was also developed, which differs from the first design in that the supply enters the valve at the center and exits radially as shown in Fig. 5.9, opposite the orientation of the first design. With this design the orifices are located on the outer diameter of the valve body and are opened or closed using a ring which is manipulated radially.

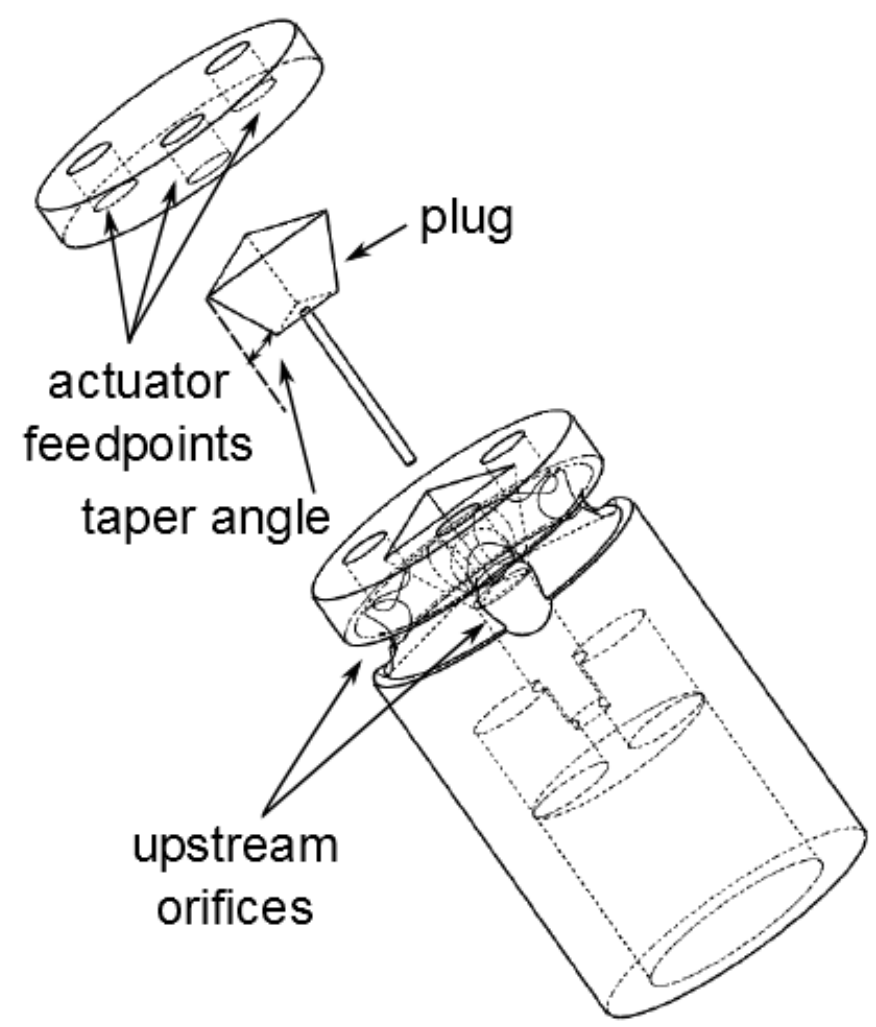

Figure 5.5: Exploded view of the plug-based valve model showing the valve body and internal components. 


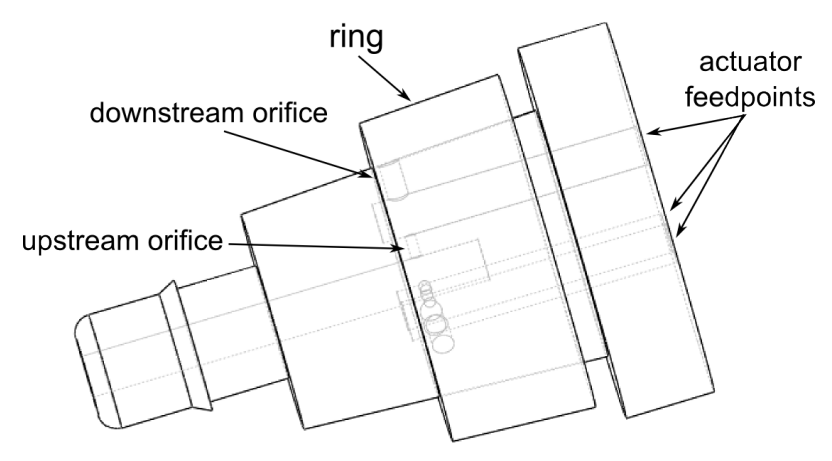

Figure 5.6: Model of ring based flapper valve design.

This valve design is normally open such that the majority of the flow bypasses the actuator and dumps to the return line. When the manipulated part (plug or ring) is activated in a particular in-plane direction, the orifices are activated differentially: the orifice(s) that the plug gets closer to become more restrictive, and the corresponding actuator pressures increase; the orifice(s) that the manipulated part gets further away from becomes less restrictive, and the corresponding actuator pressures decreases. This in turn produces a directional actuation of the manipulator to bend in the direction where the orifices become more restrictive. Out of plane movement of the manipulated part pressurizes or de-pressurizes the actuators simultaneously. This has the effect of increasing or decreasing the manipulator stiffness and also provides the ability to independently manipulate the pressure at the three actuator feed points. These orifices are sized based upon the necessary flow rate and the valve characteristics described by Eq. 5.7 as detailed in Section 6.3.

\subsubsection{Plug-Based Valve Design}

The plug-based valve consists of a single supply pressure input which feeds three upstream (fixed) orifices (Fig. 5.5). The central plug is manipulated electromagnetically (Fig. 5.7) by three independent control signals. The tetrahedral shaped 
valve plug can move both in plane and out of plane and can be manipulated to block each downstream (variable) orifice individually, in pairs of two, or all three depending on how the control signals are applied. When an orifice is blocked, pressure increases at the feed point for the corresponding actuator. Conversely, when the orifice is unblocked, flow passes to the return line and pressure at the actuator feed point decreases. Figure 5.8 shows one third of the flow path when the variable orifice is open (top) and when the orifice is blocked (bottom). In the open position, the majority of the flow passes through the valve following the least resistance path and exits to the return line. When the variable orifice is blocked, the path to the return line has a high flow resistance and thus more of the fluid flow feeds into the actuator.

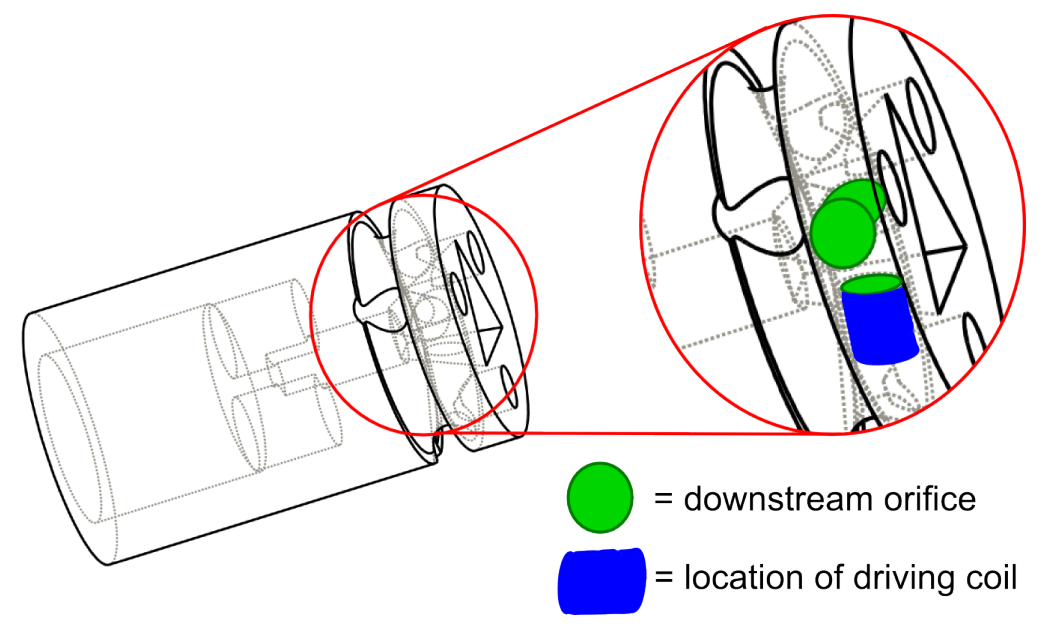

Figure 5.7: Enlarged view of plug-based valve showing downstream orifices and location of a single driving coil.

\subsubsection{Ring-Based Valve Design}

A second realization of the flapper style valve design was also developed and prototyped (see Fig. 5.6). For this version of the valve, the operation principles are the same as for the previous version. The primary difference for this design is 
123
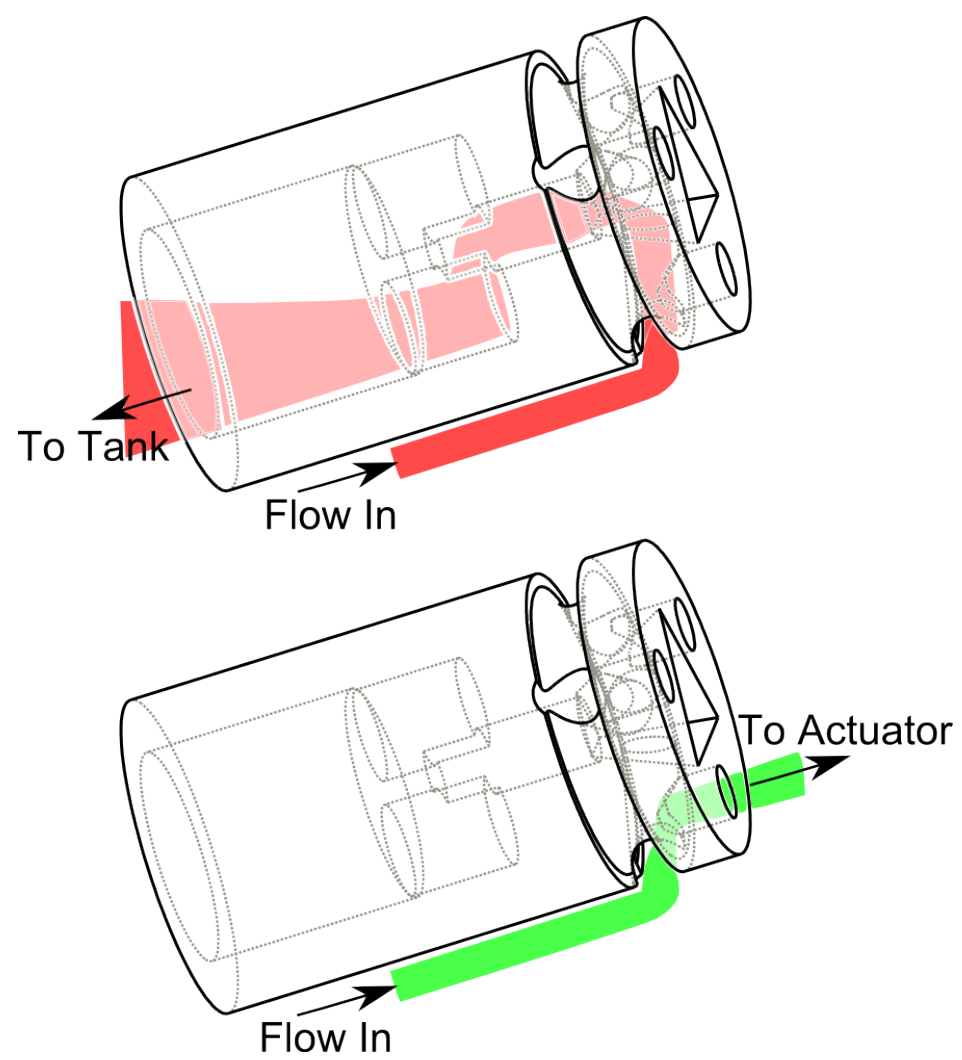

Figure 5.8: Model of the control valve showing part of the flow path for both open (top) and blocked (bottom) variable orifice conditions.

that the supply enters the valve at the center and exits radially as shown in Fig. 5.9 . opposite the orientation of the other valve version. The flapper nozzles are located on the outer diameter of the main valve body. These orifices are opened or closed differentially using a ring placed over the orifices and manipulated radially (Fig. 5.6). Further, both the valve body and the ring are tapered to allow for stiffness control of the three actuators together. With a greater taper angle, the travel distance along the long axis of the valve that is necessary for the ring to open or close the downstream orifice is reduced. This in turn results in a ring that is smaller and thus easier to manipulate. However, the smaller ring with a higher taper angle becomes more difficult to manufacture and is more sensitive 
thus requiring more precise manipulation.
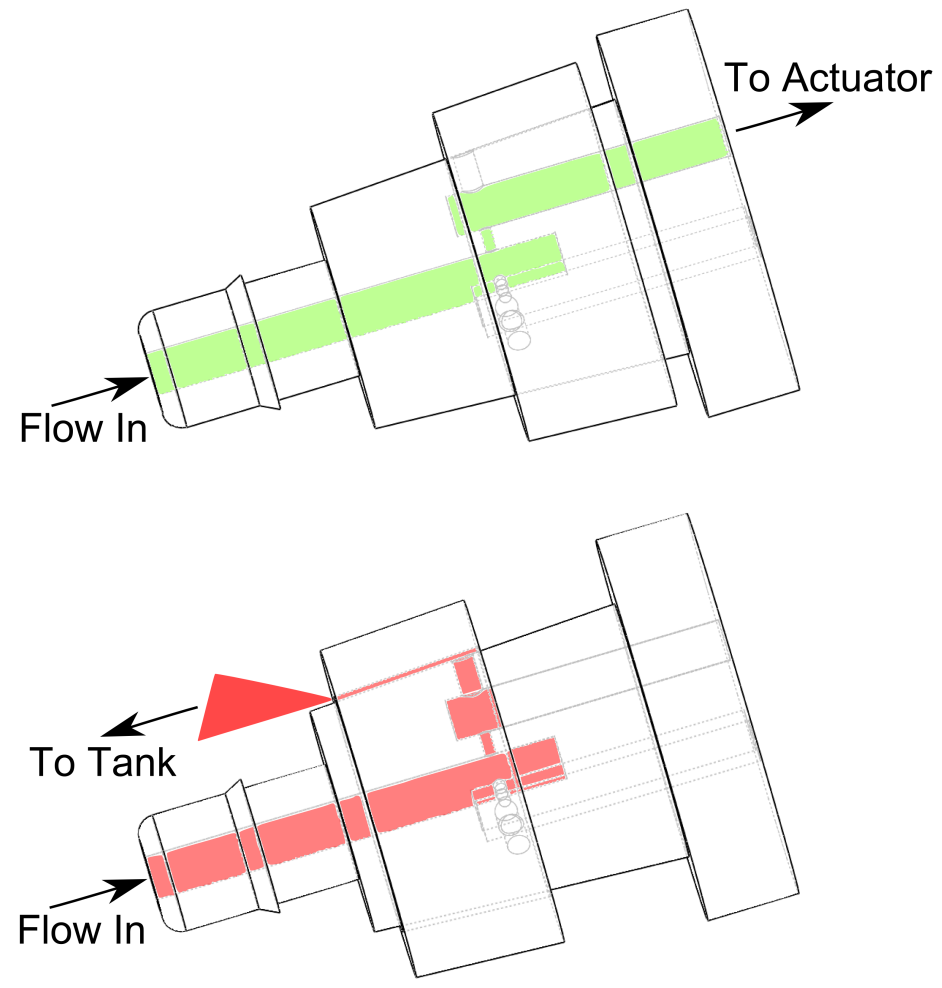

Figure 5.9: Model of the ring-based control valve showing part of the flow path for both blocked (top) and open (bottom) variable orifice conditions.

One of the primary advantages of the ring-based valve design over the plug-based design is that this design is more easily produced using conventional machining techniques. For example, it is possible to produce this design using a standard lathe.

\subsubsection{Valve Connection for Supply and Return Lines}

The design of each valve geometry is such that both the high-pressure supply and the low-pressure return lines can connect to the valve body in a direction 


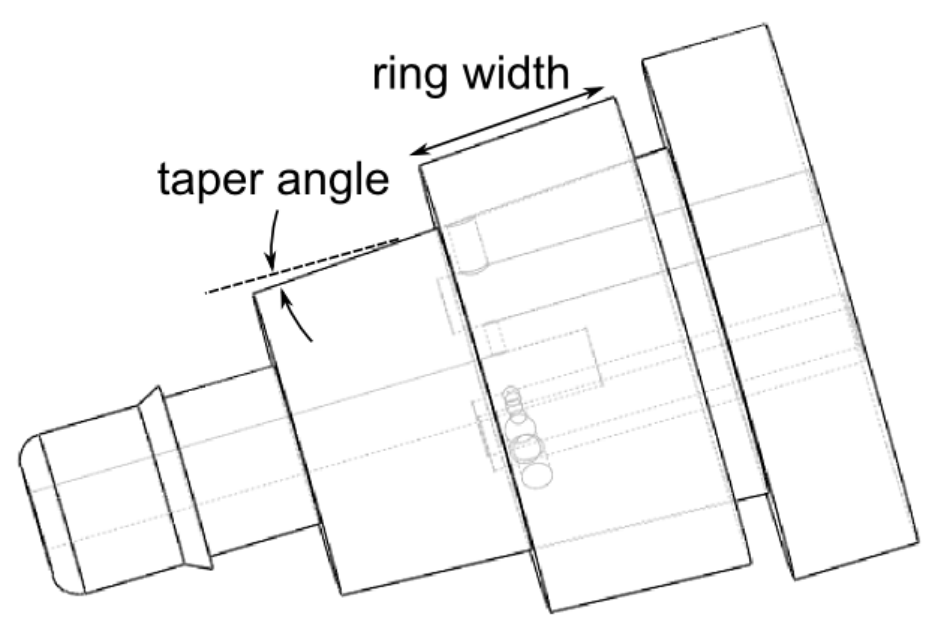

Figure 5.10: Illustration of the ring-based valve showing the taper angle.

parallel to the longitudinal axis of the valve. This method of connection keeps the overall size requirements of the valve in the radial direction as small as possible. One realization of this method of connection would employ a hydraulic line with the cross-section shown in Fig. 5.11, where the black areas represent the tubing material and the inner white areas represent the multiple lumens of the tubing. This tubing is then connected to the base of the valve body such that the outer two lumens pass over the lower portion of the valve body and are sealed to the valve at the distal outer diameter as shown in Figs. 5.12. The inner lumen passes inside the inner diameter of the base of the valve body and is sealed to this inner wall or the barbed fitting. The hydraulic supply and return then behave as shown in Figs. 5.8 and 5.9 . The use of this style of tubing permits the option of supplying the valve by running the tubing down the length of the endoscope working channel and connecting to an external pump located in the surgical suite. However, when more than one valve is placed in series within the working channel such as would be necessary with a multi-segment manipulator, providing space for the tubing from the distal valve to bypass the proximal valve may prove challenging.

Another option is to utilize the existing irrigation and suction channels common to most current endoscopes. These channels exist to facilitate surgical procedures 


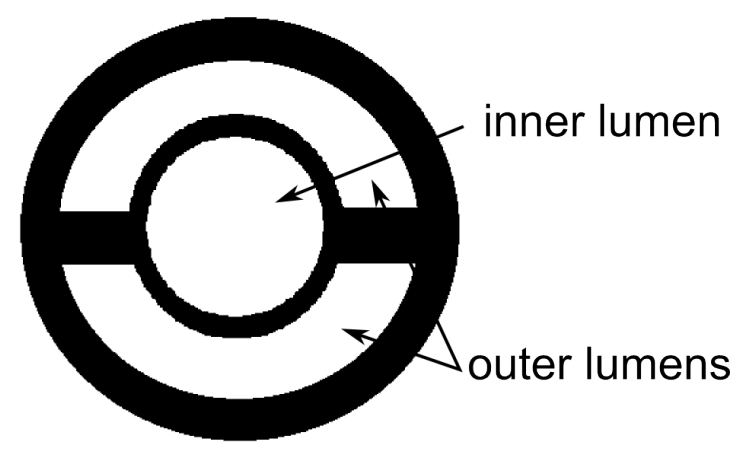

Figure 5.11: Example cross-section of a multi-lumen tubing used to provide both feed and return lines for the control valve.

by flushing the surgical site of debris and removing fluids from the site, respectively. Making use of these existing fluid channels would reduce the number of lines running the length of the endoscope and eliminate the need for a secondary external fluid pump as existing endoscopic irrigation pumps are typically capable of meeting the design requirements or could with minor modification such as increasing the available pressure [149]. 


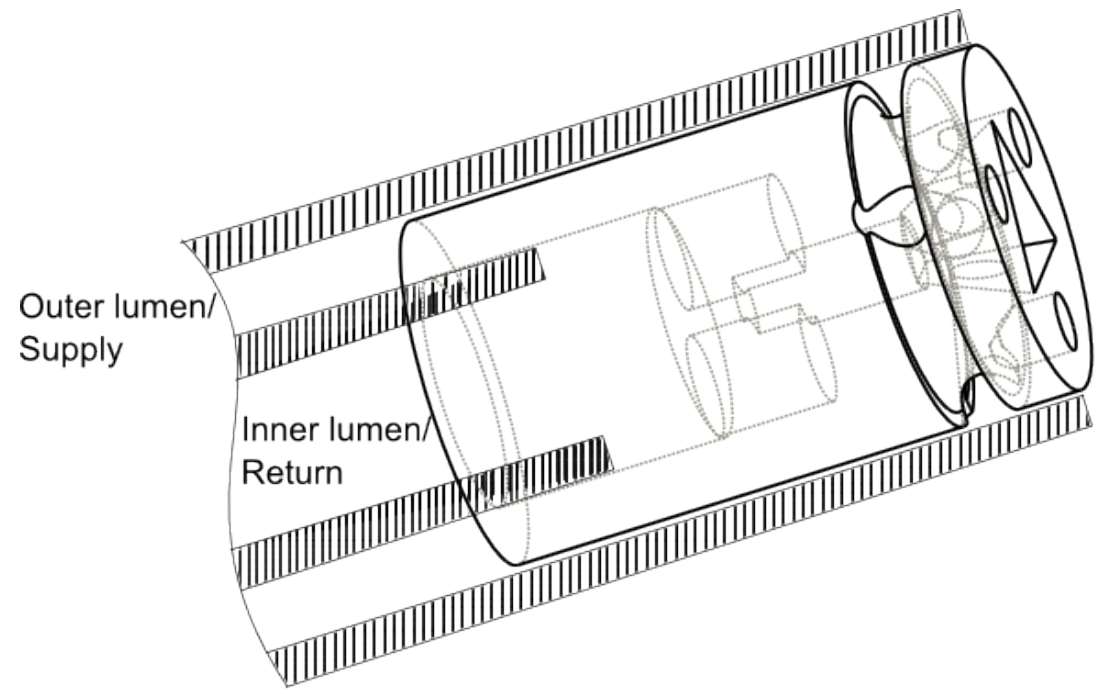

(a)

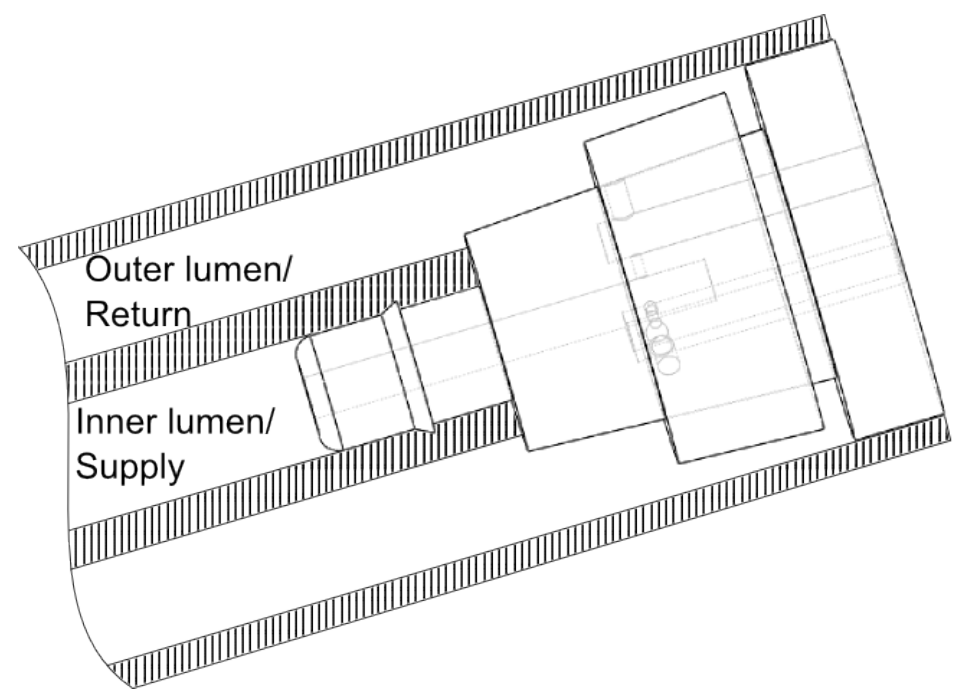

(b)

Figure 5.12: Illustration of valve connection to the hydraulic supply and return lumens of the described tubing for the (a) plug-based valve design and (b) the ring-based valve design. 


\subsection{Valve Prototyping and Experimental Results}

\subsubsection{Testing of Driving Coils}

Several driving coils were fabricated for testing the dissipation of heat from the coil under load at a scale of 2.5 times the design scale. An example of one such coil is shown in Fig. 5.4. The first coil tested had no material at the center of the coil (air core). The coil was placed near a fabricated plug made out of low-carbon steel. The coil was then energized and the gap between the coil and the plug at which the plug was pulled into contact with the coil was observed. It was found that the gap for which pull-in occurred was less than $0.25 \mathrm{~mm}$ (the designed gap size) for coil currents up to $150 \mathrm{~mA}$. At currents above $150 \mathrm{~mA}$, the coil temperature quickly exceeded allowable limits resulting in coil failure due to melting of the wire insulation. Therefore, it was concluded that a coil with an air core was not suitable for this design.

Next, a coil with a steel core material was tested under the same conditions. For the solid core coil, it was observed that the pull-in gap was approximately $1 \mathrm{~mm}$ when the coil current was $80 \mathrm{~mA}$. Further, it was observed that the temperature of the coil remained at a reasonable level (warm but not hot to the touch) even when the coil current was applied for more than 10 seconds at a time. This result suggests a much greater likelihood that plug pull-in can be achieved at coil currents which allow for proper heat dissipation. It is important to note that these tests were performed under conditions such that the primary mode of cooling was passive dissipation to the surrounding air. In practice the coil will be subject to forced flow of saline which has a much higher heat capacity.

Finally, testing of the heat dissipation of the coil was tested when the coil was submerged in room temperature water. When submerged it was found that the current in the coil could be increased to approximately $300 \mathrm{~mA}$ for several seconds 
before the coil temperature approached allowable levels. This observation confirms the notion that the current capacity of the coil is significantly higher when the surrounding fluid is water/saline rather than air.

\subsubsection{Valve Prototype Testing}

\section{Plug-Based Valve Testing}

Both versions of the valve were also prototyped and tested. The plug-based version of the valve was printed using a Stratasys Dimension 1200es rapid prototyping machine at three times geometric scale such that the outer diameter was $18 \mathrm{~mm}$. The orifices were scaled according to the flow characteristics (Section 5.3) such that the downstream orifice was $1.3 \mathrm{~mm}$, upstream orifice was $0.74 \mathrm{~mm}$, and the gap was $0.4 \mathrm{~mm}$. A scaled prototype was selected due to difficulties associated with fabrication at the design scale (Fig. 5.13) for which the outer diameter is $6 \mathrm{~mm}$, downstream orifice was $0.7 \mathrm{~mm}$, upstream orifice was $0.4 \mathrm{~mm}$, and gap was $0.25 \mathrm{~mm}$. Shown in Fig. 5.14, one version of this valve prototype was made to be manually manipulated (Fig. 5.14a). To achieve this, a hole was drilled in the top cap of the valve to provide access to the inner plug. This hole is located downstream of the primary outlets at the same location as the intended return line such that the inclusion of this hole did not affect valve operation. A second prototype with a sealed cap was also produced (Fig. 5.14b). Included within this prototype was three electromagnetic coils intended to provide manipulative force to the inner plug. Each of these prototypes were fitted with a cylindrical ring to seal the outer supply channel and tubing to provide the necessary connections to the supply, return, and actuators. Each of these connections was rigidly sealed using steel reinforced epoxy (J-B Weld).

Pressure to the valves was supplied using a rotary vane pump (Procon) driven by an electric motor (Fig. 5.15). A relief valve was used to regulate the supply 


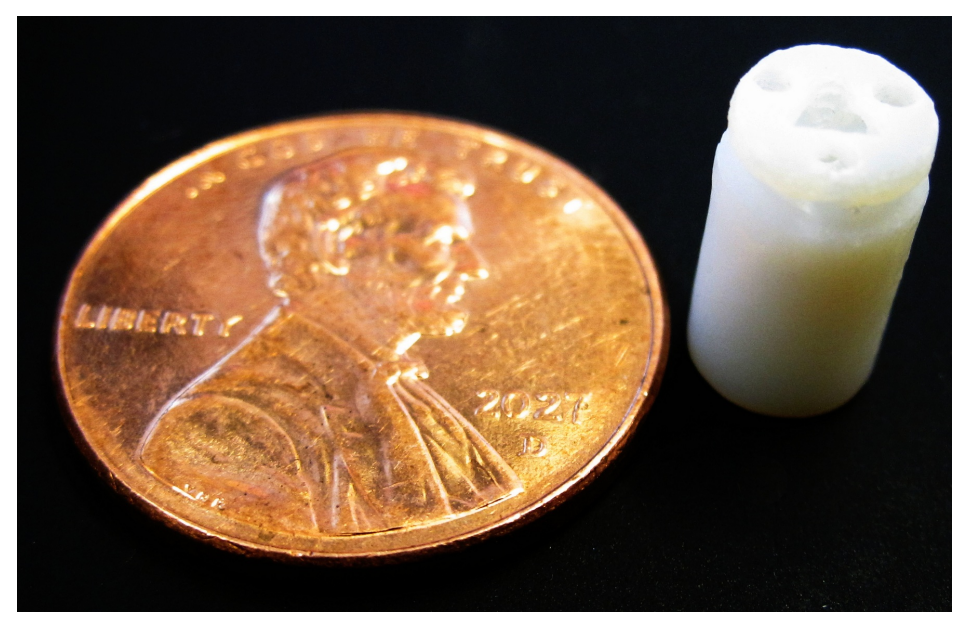

Figure 5.13: Photograph of the design scale prototype of the plug-based valve design.

pressure at a steady $689 \mathrm{kPa}$. The flow rate from the pump was sufficient to provide constant pressure to the valves.

Manual manipulation of the valve plug demonstrated that it was possible to vary the pressure at the feed-points to each of the outlets that feed the actuators. However, the increase in pressure at the feed-point was not as large as was expected. Direct measurement of the pressure was not possible; however, estimations based on actuator inflation suggest a pressure of approximately $170 \mathrm{kPa}$, or $25 \%$ of the supply pressure, was achieved. From Fig. 5.3, it should be possible to achieve at least $90 \%$ of the supply pressure using the flapper valve. Possible causes for this may be poor geometric tolerances in the printing of the valve body or in the valve plug resulting in poor surface contact. In particular, the imprecision in the inside corners of the plug seat may have restricted the plug from closing the gap. Further, accessing the plug through the hole in the cap proved difficult and provided a limited range of motion. Using the electromagnetically manipulated valve in the same configuration produced similar results. A small change in feed-point pressure was observed but the change was not significant enough to produce any 


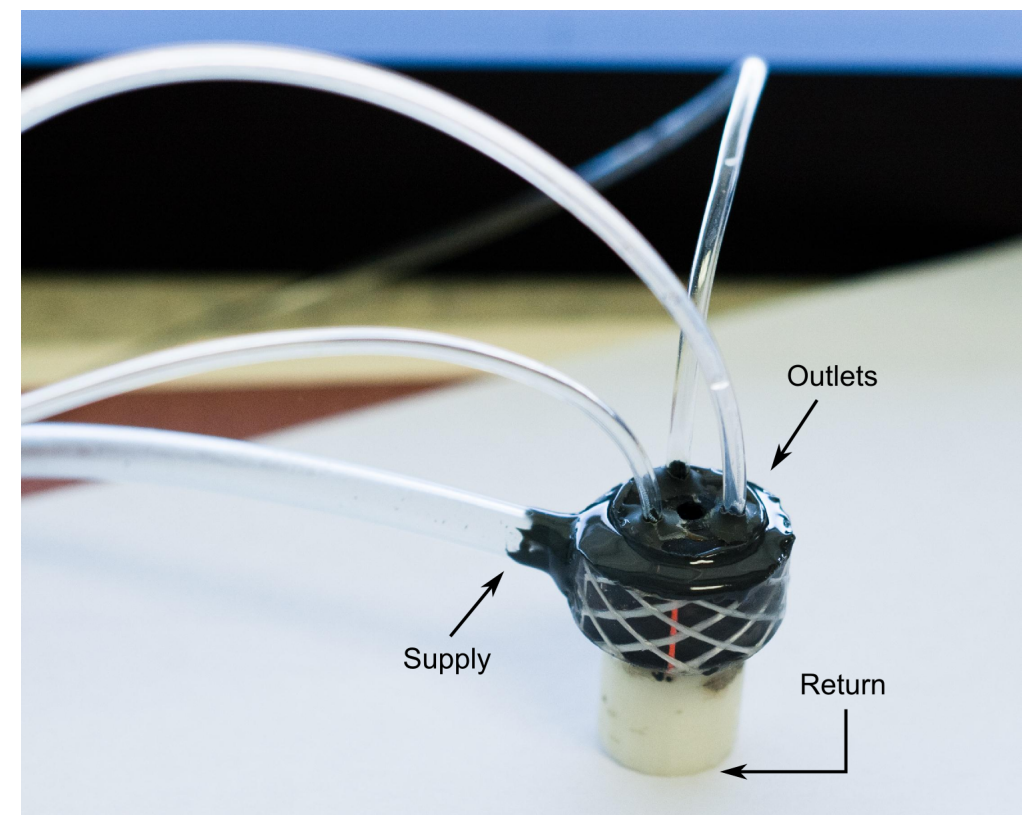

(a)

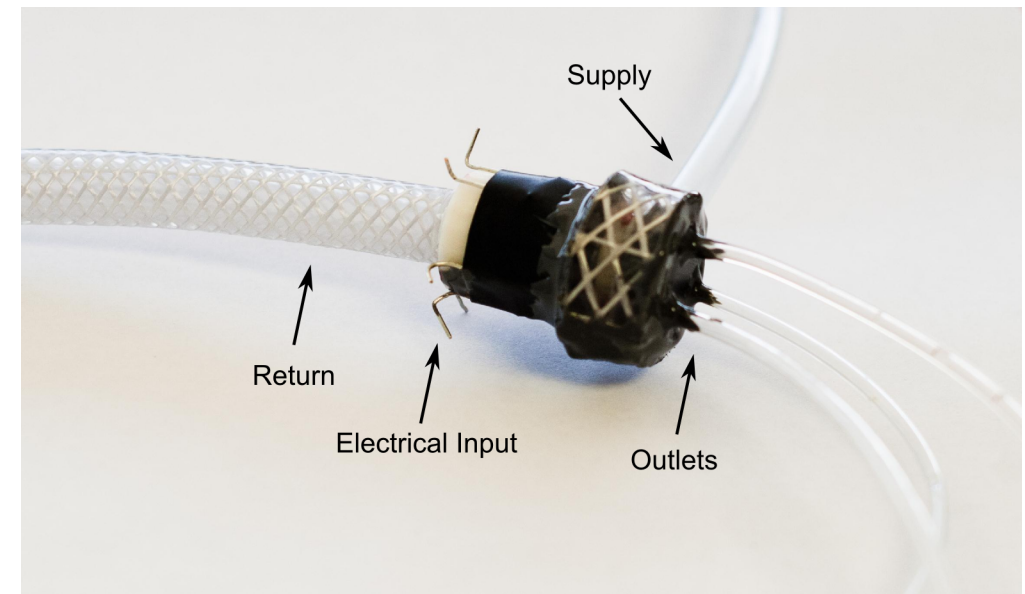

(b)

Figure 5.14: Photographs showing the scaled plug-based valve prototype in both (a) manually manipulated and (b) electromagnetically manipulated configurations. 


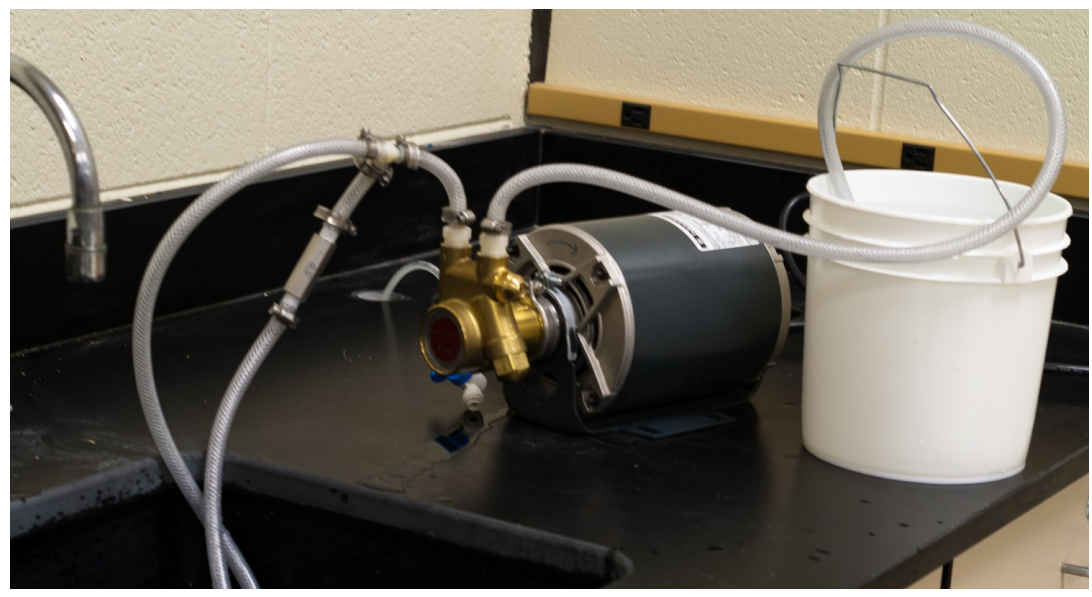

Figure 5.15: Photograph of hydraulic supply setup.

significant output at the actuator; an estimated pressure of $100 \mathrm{kPa}$ or $15 \%$ of the supply pressure. This result may be due in part to higher flow forces at the valve orifice for the three times scale version of the valve. Increasing the diameter of the valve orifice significantly increases the flow forces at the valve plug. It is difficult to predict any additional reasons for the poor performance of the valve since it was not possible to observe the internal valve operation during testing. Future exploration of this valve design should include developing a means of investigating the internal operation of the valve to locate any flaws in the design or fabrication of the prototype.

\section{Ring-Based Valve Testing}

The ring-based valve design was also prototyped and tested. However, to accommodate the need for manual manipulation and to reduce manufacturing costs, the scale of the valve was increased. At prototype scale, the outer dimension was 20 $\mathrm{mm}$ and the overall length was $32.5 \mathrm{~mm}$. An upstream orifice diameter of 0.74 $\mathrm{mm}$ was used to provide the desired flow rate and then Eq. 5.7 resulted in a downstream orifice diameter of $1.3 \mathrm{~mm}$ in the fully open condition. Using this 
diameter, the necessary gap, $g$, between the orifice and the flapper under the fully open condition is $0.4 \mathrm{~mm}$. Also, for this prototype, a taper angle of $3^{\circ}$ was used in order to make manual manipulation of the valve ring possible. A prototype of this design is shown in Fig. 5.16. The prototype was produced using CNC machining techniques (Island Tool \& Die, Pine Island, MN) and connected to the hydraulic lines using steel reinforced epoxy (J-B Weld) and a hose clamp, as shown in Fig. 5.17.

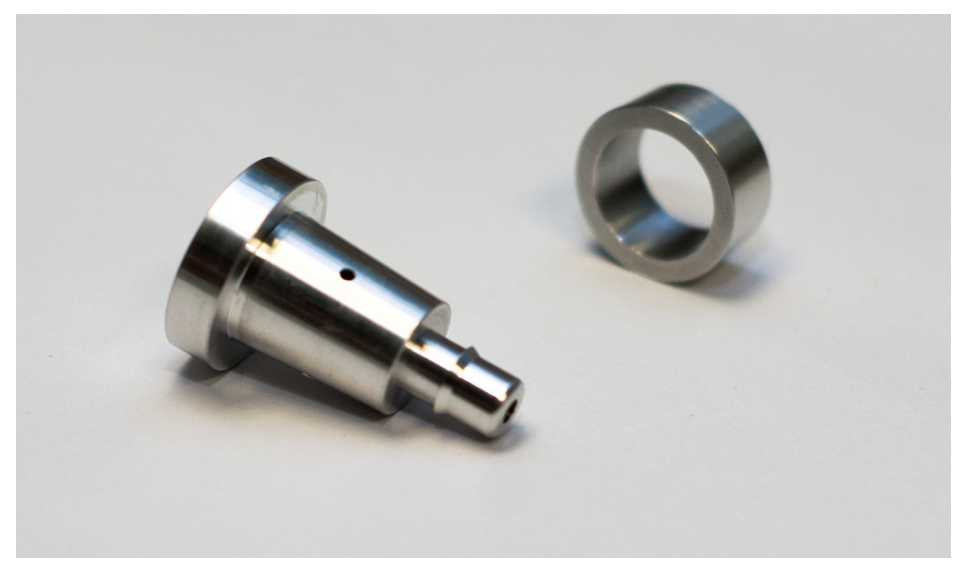

Figure 5.16: Machined prototype of the second valve design.

The ring-based valve prototype was tested using the same setup shown in Fig. 5.15. Testing of this prototype involved manual manipulation of the outer control ring to differentially open and close the flapper nozzles. It was found that the prototype of the ring-based design performed significantly better than the first design. It was possible to inflate artificial muscle actuators connected at the actuator feed points to their fully contracted states and thus the pressure increases at these locations were demonstrably superior than was observed for the first valve design, on the order of $650 \mathrm{kPa}$ or $95 \%$ of the supply pressure. Further, it was possible to simultaneously increase the actuator outlet pressures in pairs by positioning the ring such that two orifices were sealed. Finally, it was found that the relative stiffness of the three outlets together could be manipulated by positioning the ring up or down the taper. The effectiveness of this valve design is 
also explored in Section 6.4.3 where it is combined with the rest of the manipulator components to evaluate overall performance.

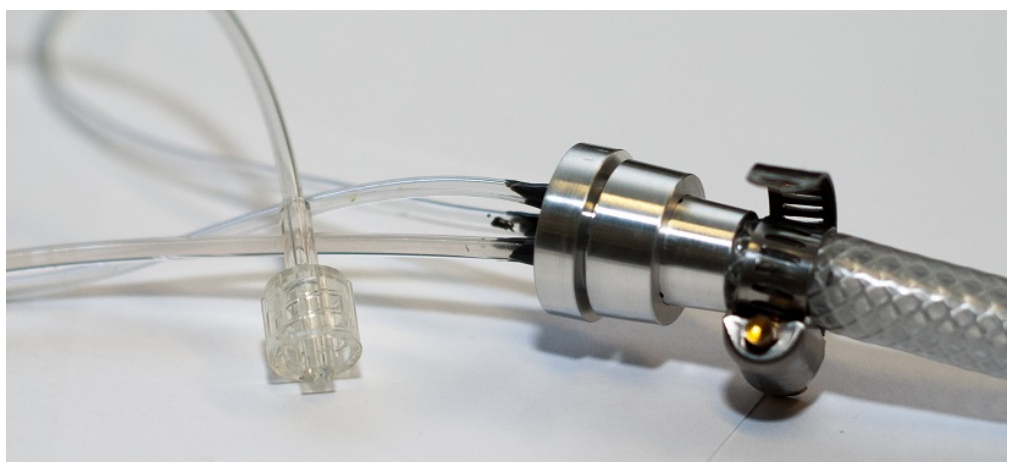

(a)

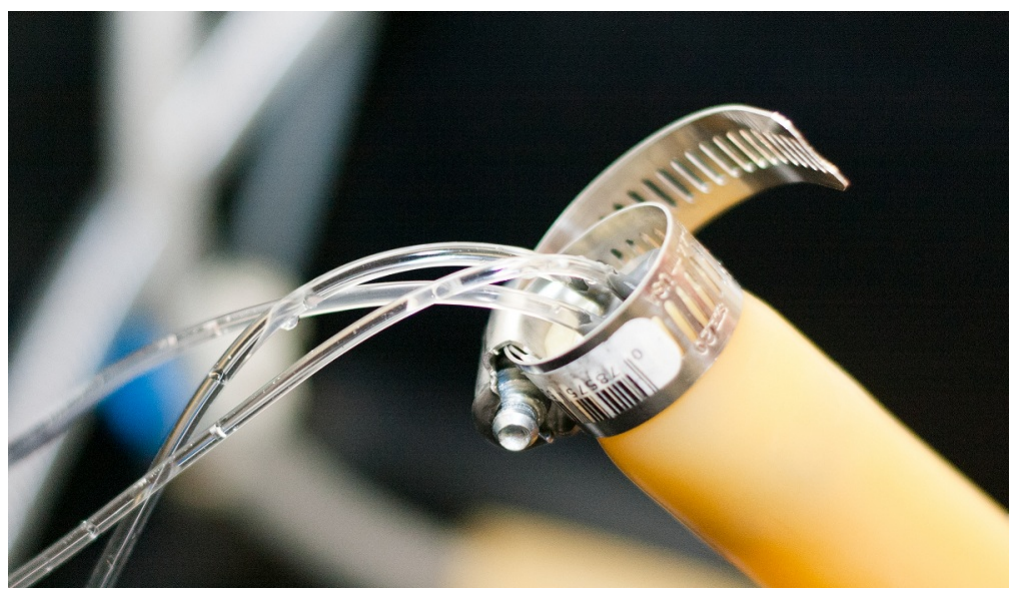

(b)

Figure 5.17: Photographs showing the scaled valve prototype for the ring-based design with (a) outlet and supply lines attached and (b) assembled within the return line. 


\subsection{Summary of Control Valve Design}

After review of the available valve technologies for control of a hydraulic system, no options were found at the scale necessary for this application. The design presented here is based on a single-jet flapper valve that facilitates control over the ratio of actuator pressure to supply pressure. The flapper valve design provides benefits of a relatively linear performance curve on the basis of outlet pressure versus downstream orifice size as shown in Fig. 5.3 [148]. The theoretical behavior of the valve is based on the principles of pressure drop across a flow restriction. As such, these predictions are dependent upon the quality and accuracy of the valve fabrication processes which can introduce unexpected errors due to poor surface finish or dimensional tolerances. For smaller valve orifices, these errors can become more pronounced. The valve design presented here consists of three

such flapper nozzles for the control of three actuators. Two possible realizations of this design were discussed. Each realization contained a manipulated component (plug or ring) that plays the role of the flapper for each of the actuators, differentially opening and closing the three nozzles through planar motion. Further, axial motion of the plug or ring controls stiffness of the three actuators together.

Testing of the electromagnetic driving coils showed that the coil was able to manipulate the plug, when a steel core was placed at the center of the winding, without exceeding the fusion temperature of the wire. Further, when tested while submerged in water, it was possible to increase the coil current nearly fourfold without shorting, greatly exceeding the rated current capacity of the wire. This suggests that the coil actuator provides a suitable method for plug manipulation in a submerged condition.

Finally, it was found during repeated attempts at fabricating a workable prototype of the plug-based valve design presented here that manual manipulation of the valve produced a muted effect on the pressure at the actuator feed-point. 
Findings were similar for the powered valve. The likely cause of this result is poor surface finish in the fabricated valve due to the rapid prototyping process used for fabrication. The ability to properly seal the nozzle exit is important for flapper valve operation. Testing of the ring-based valve design revealed that this design performed significantly better than the first under similar operating conditions. It was possible to increase the pressure at the actuator feed points individually, in pairs, or all at the same time. This result can likely be attributed to the fact that the prototype of this version could be fabricated using conventional techniques. This allowed for a better surface finish and thus a superior seal at the flapper nozzle exit. These results indicate that this valve design represents a feasible solution for the control valve at design scale. 


\section{Chapter 6}

\section{System Assembly}

As the final chapter describing the design of the surgical manipulator, this chapter focuses on the assembly of the various components that form the surgical manipulator. First, in Section 6.2 the models of each of the components are collected and assembled into a system model. This system model can be used to provide some insight into predicted manipulator performance and outcomes. Next, in Section 6.3 the design specifications for each of the components are provided to quantify what the manipulator would look like at design scale in such a way that it would be capable of meeting the design requirements. In Section 6.4, the system is analyzed using the analytical models. Further, a system prototype is then presented which has been scaled up from the intended design scale. This prototype is used to evaluate some of the design metrics and provide motivation for future miniaturization towards a design scale prototype. 


\subsection{Motivation for System Level Analysis}

As discussed in Section 1.1.5, there are a number of surgical robot concepts being explored, each with strengths and weaknesses. As a means of producing a relevant comparison against these other solutions, it was necessary to develop an assembled model of the manipulator as a system. By coupling the system sub-components together as designed, it is possible to analyze the theoretical system performance and identify areas of possible improvement. These results can also be compared against the design criteria set forth in Chapter 2 in terms of system performance. Further, the system model can enable future iteration of the design process for each of the sub-components whenever there is a change in design requirements or application. As a whole, the system model permits virtual assessment of system performance for control system design.

\subsection{Analytical Description}

To develop a model of the assembled system, each of the components must be assembled together. The system components are coupled together through both hydraulic and geometric relationships. The control valve supplies pressure directly to the actuators. Each actuator mounted in its elongated configuration to allow for antagonistic stretch and is coupled to a tendon which in turn is routed along the manipulator backbone. Backbone shape directly determines relative length of each actuator. In both the two-dimensional and three-dimensional cases, only a single manipulator segment is modeled. Future work should build upon this and include the ability to model multiple manipulator segments.

For both the two-dimensional and three-dimensional cases, it is assumed that the number of disks along the actuator tendons are sufficiently large such that the angle $\beta(i)$ between the tendon and each intermediate disk $i$, as shown in 
Fig. 6.1, is sufficiently small so that each tendon can be considered to be normal to the disk. Further, it is also assumed that any frictional interaction between the tendon and the disks is negligible and thus tension within each tendon is efficiently transmitted through each intermediate disk. These two assumptions dictate that the interaction between the tendons and the intermediate disks have negligible effects on the beam shape [87]. As such, the contractile force generated by each AMA is transmitted to the terminal disk by each associated tendon and the tendons present loading on the beam only via the terminal disk, in the form of a force directed along the line of action of the tendon and perpendicular to the terminal disk as shown in Fig. 6.2, but not via the intermediate disks. Because the tendons are attached to the terminal disk at an offset distance from the disk center, the net result is an applied moment and an applied force which are counteracted by the moment and force produced by the beam through deformation.

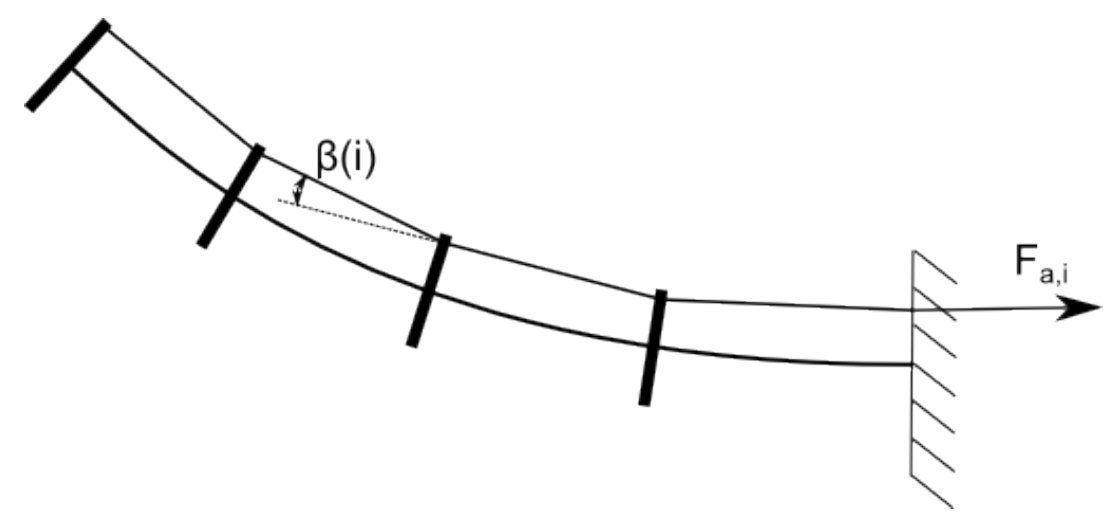

Figure 6.1: Graphic showing angle change of tendon due to routing through intermediate disks.

\subsubsection{System Model in Two Dimensions}

In the two-dimensional case (Fig. 6.3), the individual system component models can be coupled together as shown in Fig. 6.4. The two-dimensional system includes one valve consisting of two active orifices and two passive orifices, two 


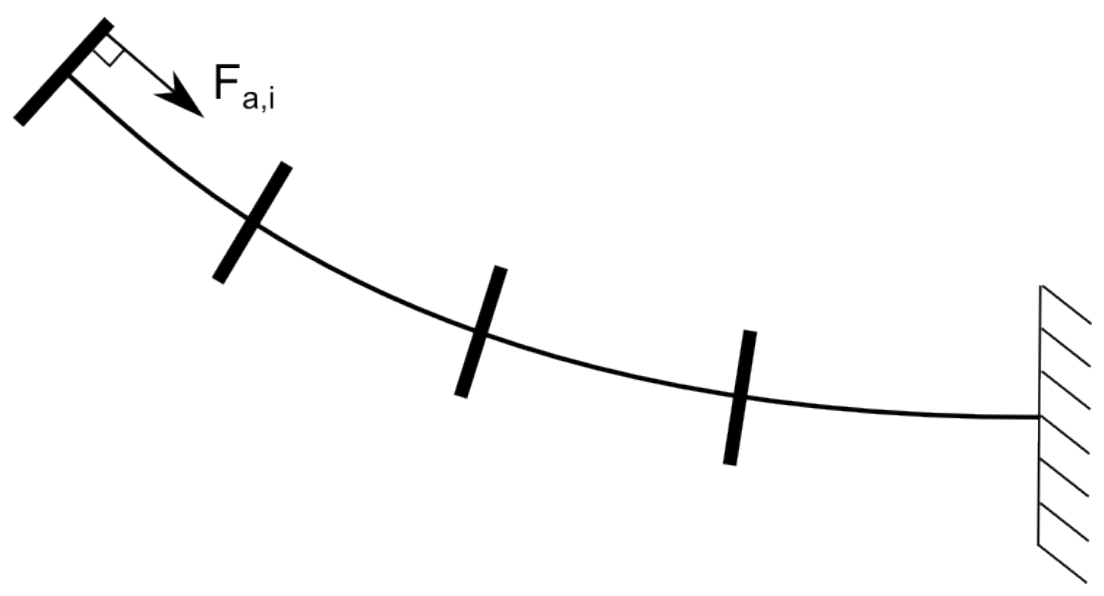

Figure 6.2: Graphic showing resulting free body diagram for assumption of many intermediate disks.

actuators, and a single segment beam that can exhibit planar motion. The twodimensional beam model assumes planar bending and does not take into account any deformation due to beam mass or the effects of shearing or axial extension. For the overall system model, the control input is the size of the gaps for the valve's variable orifices. Since the two-dimensional model incorporates two antagonistic actuators, only two active valve orifices are included, which operate differentially, in addition to the two upstream fixed orifices. The first block in Fig. 6.4 is the valve hydraulics block. This block accepts the control input, $g$, and the time derivative of the actuator lengths, $\dot{L}_{a}$. From these inputs, the pressure at the actuator feed points, $P_{a}$, are calculated using Eq. 5.6 (repeated below in Eq. 6.1), where $Q_{a, i}$ is calculated as shown in Eq. 6.2. The actuator pressures then act as the input to the actuators block along with the actuator lengths, $L_{a}$. Using Eq. 4.11 (repeated in Eq. 6.3) for each of the actuators, the load from each of the actuators is calculated as $F_{a, 1}$ and $F_{a, 2}$. Recalling that when the actuators are stretched from their nominal length, the force load due to the actuator stretch is modeled as a spring force $\left(F_{s}=k \cdot \Delta L_{a}\right)$ which results from stretching the inner bladder, as discussed in Section 4.2. Under nominal conditions when the valve plug is centered, the actuator pressure is $66.4 \mathrm{kPa}$ as defined by the ratio of the 
downstream and upstream orifice diameters.

$$
\begin{gathered}
P_{a, i}(t)=\left[\rho Q_{a, i}^{2}+2 A_{0}^{2} C_{d 0}^{2} P_{s}-\frac{2 \rho A_{0} C_{d 0} Q_{a, i}}{A_{0}^{2} C_{d 0}^{2}+A_{f, i}^{2} C_{d f}^{2}}\left(A_{0} C_{d 0} Q_{a, i}\right.\right. \\
\left.\left.+A_{f, i} C_{d f} \sqrt{\frac{2 P_{s} A_{0}^{2} C_{d 0}^{2}+2 P_{s} A_{f, i}^{2} C_{d f}^{2}-\rho Q_{a, i}^{2}}{\rho}}\right)\right]\left(2 A_{0}^{2} C_{d 0}^{2}+2 A_{f, i}^{2} C_{d f}^{2}\right)^{-1}
\end{gathered}
$$

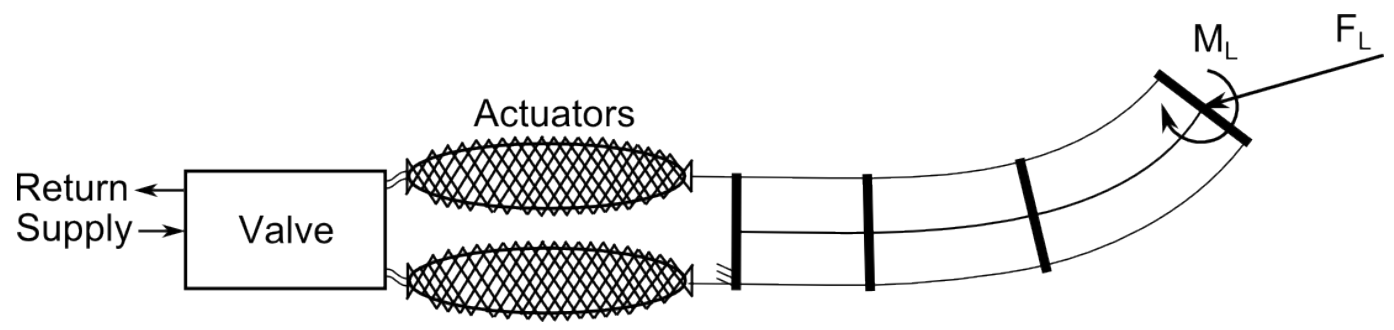

Figure 6.3: Skeleton model showing the basic components that make up the twodimensional system.

The quantities relating to the actuator length, $L_{a}$ and $\dot{L_{a}}$, which act as inputs to the actuators and valve hydraulics blocks, respectively, originate from the beam mechanics block. The beam mechanics block takes as input the position and orientation of the distal end of the manipulator, $[x, z, \theta]$, which is initialized in the undeformed configuration. From this information, the actuator length can be described as a function of the orientation of the distal end of the manipulator. This relationship can be found by examining a differential segment of the manipulator (Fig. 6.5 [69]. If $d s$ represents the arc length of the manipulator backbone 


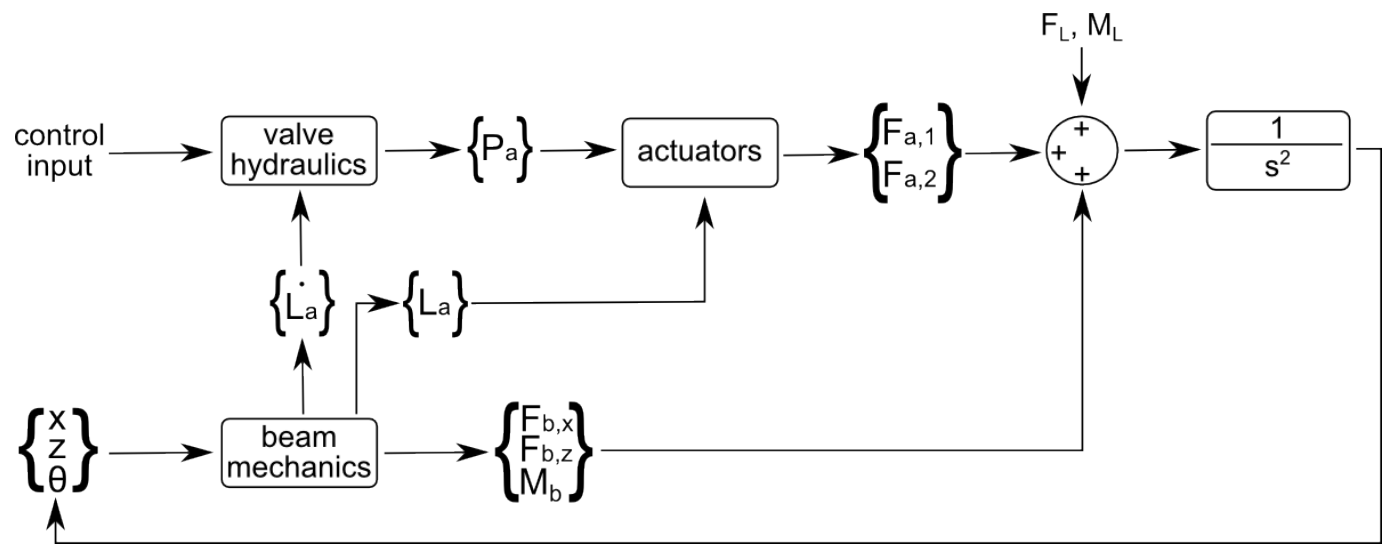

Figure 6.4: Simplified block diagram of system model.

over this segment and the corresponding arc length of the location of tendon attachment is defined as $d s^{*}$, then the relationship between the two arc lengths can be found by first writing

$$
d s^{*}=(\rho-r) d \theta
$$

where $r$ is the distance from the center of the backbone to the compressive side tendon, $\theta$ is the angular orientation of the backbone, and $\rho$ is the radius of curvature of the manipulator segment which can be defined as $(d \theta / d s)^{-1}$. Rewriting this expression to get

$$
d s^{*}=d s-r \cdot d \theta
$$

and then rearranging and integrating both sides over the length of the beam, $s \in[0, L]$ results in an expression for the total length of the tendon, $s^{*}(L)$, as a function of the beam length, $s(L)$, and the orientation of the beam at $s=L$, $\theta(L)$, as shown in Eq. 6.6

$$
s^{*}(L)=s(L)-r \theta(L)
$$

Thus, the change in length of a given tendon located at the outer edge of the manipulator is equal to $r \theta(L)$. Therefore, by knowing the orientation of the 
terminal disk, the corresponding tendon position and thus actuator length can be calculated. Note here that the tendon is assumed to be inextensible. The change in tendon length that is being calculated is really the portion of the tendon within the flexible section of the manipulator. Because the actuator is connected to the terminal disk of the backbone via the tendon, the length of the actuator, $L_{a}(t)$ can be determined by the change in the tendon length as the manipulator bends. The actuator length is given by:

$$
L_{a}(t)=L_{a, 0}-r \cdot \theta(t, L)
$$

where $L_{a, 0}$ is the length of the actuator when the manipulator is straight (i.e. $\theta(s)=0$ for all $s \in[0, L])$, and $r$ is the radius of the routing disk at the point of the tendon connection. For the other actuator the same relationship exists but instead the change in length, $r \theta(L)$, is added to the initial length for positive values of $\theta(t, L)$.

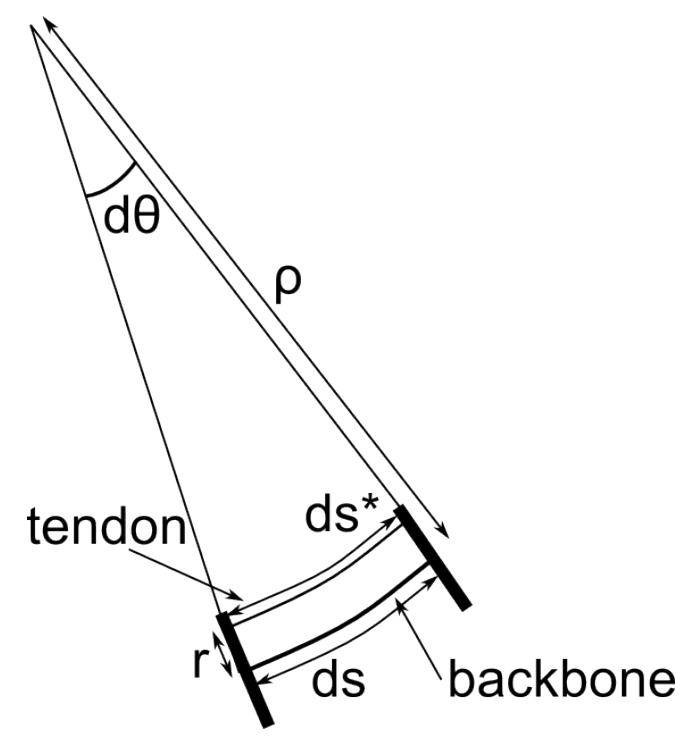

Figure 6.5: A differential segment of the manipulator with the backbone and tendon shown.

As it is assumed that an end-effector is placed at the distal end of the manipulator, 
translational and rotational inertial dynamics allow for calculation of the location and orientation of the distal end of the manipulator due to the applied control. A force and moment balance is applied to the end-effector mass, treated as a free body as shown in Fig. 6.6. Loads applied to the end mass in the global coordinate system originate from the actuators, the reaction load from the backbone, $F_{b}$ and $M_{b}$, and from any external loading, $F_{L}$ and $M_{L}$, due to tissue manipulation or collision, as shown in Eqs. 6.8-6.10.

$$
\begin{gathered}
\sum F_{x}=m \ddot{x}=F_{b, x}+F_{L, x}+F_{a 1, x}+F_{a 2, x} \\
\sum F_{z}=m \ddot{z}=F_{b, z}+F_{L, z}+F_{a 1, z}+F_{a 2, z} \\
\sum M=I \ddot{\theta}=-F_{a 1} \cdot r+F_{a 2} \cdot r+M_{b}+M_{L}
\end{gathered}
$$

The result of these equations are values for the cartesian components of the translational acceleration, $\ddot{x}$ and $\ddot{z}$, and the rotational acceleration, $\ddot{\theta}$, which can be integrated twice to find the position and orientation of the distal end of the manipulator.

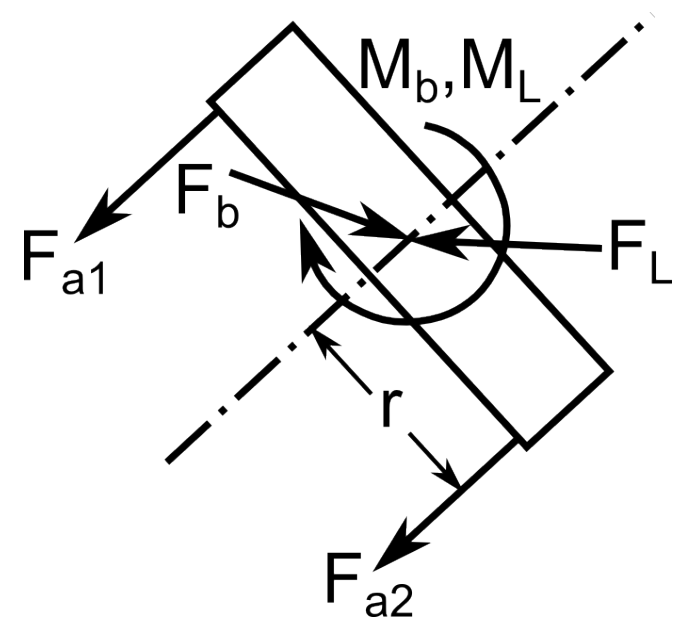

Figure 6.6: Free body diagram for mass at the distal end of the manipulator.

Further, using the model described in Section 3.3.1 for a given position and orientation, the beam mechanics block outputs the beam forces and moment acting 
on the end effector at the distal end of the beam can be described by Eq. 3.23 , copied below.

$$
F_{b, x}=\frac{\partial P E^{*}}{\partial x_{\text {end }}}, \quad F_{b, z}=\frac{\partial P E^{*}}{\partial z_{\text {end }}}, \quad M_{b}=\frac{\partial P E^{*}}{\partial \theta_{\text {end }}}
$$

\subsubsection{System Model in Three Dimensions}

For the three dimensional model of the system, the components that make up the assembled tool are the same as those for the two dimensional system. However, in order to achieve non-planar motion of the manipulator, a total of three muscle actuators and three tendons are used. Since the tendons are connected to the terminal disk in such a way that the tendon loads are applied in a direction parallel to the manipulator backbone, the resulting load on the terminal disk due to the tendon loads is realized as a moment in two Cartesian directions ( $\mathrm{X}$ and $\mathrm{Y}$ ) and one force (Z-direction) as shown below

$$
\left[\begin{array}{l}
F_{z} \\
M_{x} \\
M_{y}
\end{array}\right]=\left[\begin{array}{ccc}
1 & 1 & 1 \\
0 & 0.866 \cdot r & -0.866 \cdot r \\
-r & 0.5 \cdot r & 0.5 \cdot r
\end{array}\right]\left[\begin{array}{c}
F_{a, 1} \\
F_{a, 2} \\
F_{a, 3}
\end{array}\right]
$$

where the actuator loads, $F_{a, i}$, which are transmitted by the tendons, are connected to the terminal disk as shown in Fig. 6.7. Thus for a given manipulator segment, three degrees of freedom can be manipulated by the three actuators. The addition of more actuators would not provide control over any additional degrees of freedom. Therefore, due to the limitation on available space, the use of three active inputs for a given manipulator segment was selected for this design. These three actuator/tendon combinations are arranged equidistant around the circumference of the manipulator as shown in Fig. 6.7. 


\section{Neutral}

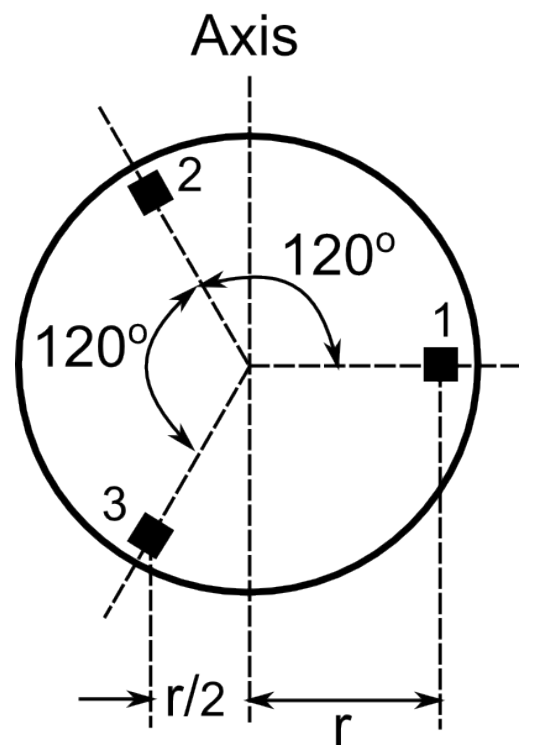

Figure 6.7: Illustration of the terminal disk showing the tendon connection points as indicated by the black squares.

The system model in three-dimensions is arranged much the same as the twodimensional case described in Section 6.2.1. The key difference being that there are six variables necessary to describe the position and orientation of the distal end of the manipulator rather than three. The modified system block diagram is shown in Fig. 6.8. The first block in Fig. 6.8 is again the valve hydraulics block. This block accepts as its inputs the control inputs, $g_{i}$, and the time derivative of the actuator lengths, $L_{a, i} \cdot$. From these inputs, the pressure at each of the three actuator feed points, $P_{a, i}$, are calculated using Eq. 6.1. The actuator pressures then act as the input to the actuators block along with the actuator lengths, $L_{a, i}$. Using Eq. 6.3 for each of the actuators, the load from each of the actuators is calculated as $F_{a, 1}, F_{a, 2}$, and $F_{a, 3}$. Again, when the actuators are stretched from their nominal length, the force load from the actuator is modeled as a spring force which results from stretching the inner bladder.

The quantities relating to the actuator lengths, $L_{a, i}$ and $\dot{L}_{a, i}$, which act as inputs 


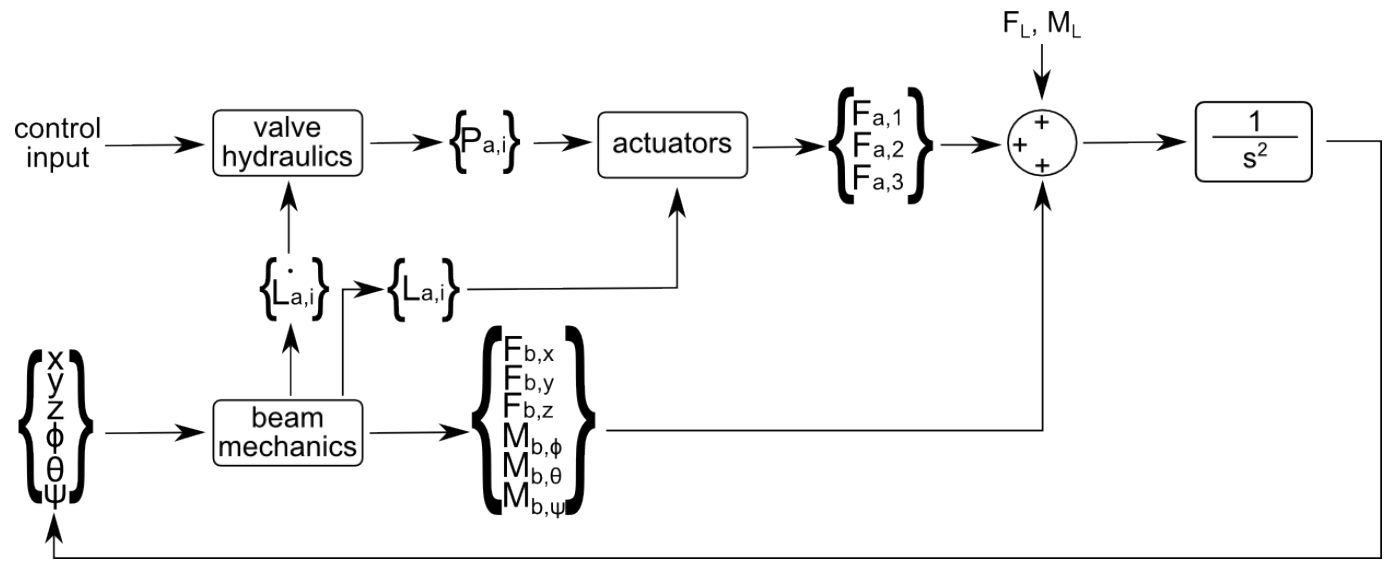

Figure 6.8: Simplified block diagram of the 3D system model.

to the actuators and valve hydraulics blocks, respectively, originate from the beam mechanics block. The beam mechanics block takes as input the position and orientation of the distal end of the manipulator, $[x, y, z, \phi, \theta, \psi]$, which is initialized in the undeformed configuration. From this information, the actuator length can be described as a function of the orientation of the distal end of the manipulator. This relationship is similar to what was shown in Eqs. 6.4-6.7. However, in the three-dimensional case, the orientation angle necessary to compute the change in actuator length is not simply $\theta$. Instead it is necessary to determine the change in orientation of a vector, $\vec{r}_{i}$, which describes the location of the tendon termination point for each tendon relative to the center of the backbone as shown in Fig. 6.10.

The arc length derivative of the $R(s)$ rotation matrix (Eq. 3.24) can then be used to operate on the reference position vector, $\vec{r}_{i, 0}$, for each of the tendon termination points and determine the change in orientation of $\vec{r}_{i}$ in the deformed state by substituting in the values which describe the orientation of the distal end of the manipulator, $\phi(s), \theta(s)$, and $\psi(s)$, for a differential segment as shown in Fig 6.9. For each differential segment, the length of the tendon can be found using the change in centerline position from one end of the segment to the other, $\vec{x}(s)$, the components of which are shown in the integrands of Eqs 3.32 - 3.34, along with 
the change in position of the tendon termination points, $\vec{r}_{i}$, as shown in Eq. 6.13.

$$
\frac{d \vec{L}_{T, i}}{d s}=\vec{x}(s)^{\prime}+R(s)^{\prime} \vec{r}_{i, 0}
$$

Integrating the magnitude of this vector over the entire beam then yields the resulting length, $L_{T, i}$ of each of the three tendons under the given beam shape. This tendon length can then be compared against the undeformed tendon length such that the change in length of the attached actuator, $L_{a, i}$ can be calculated as

$$
L_{a, i}(t)=L_{a, 0}+\Delta L_{T, i}
$$

where $L_{a, 0}$ is the actuator length in the reference configuration.

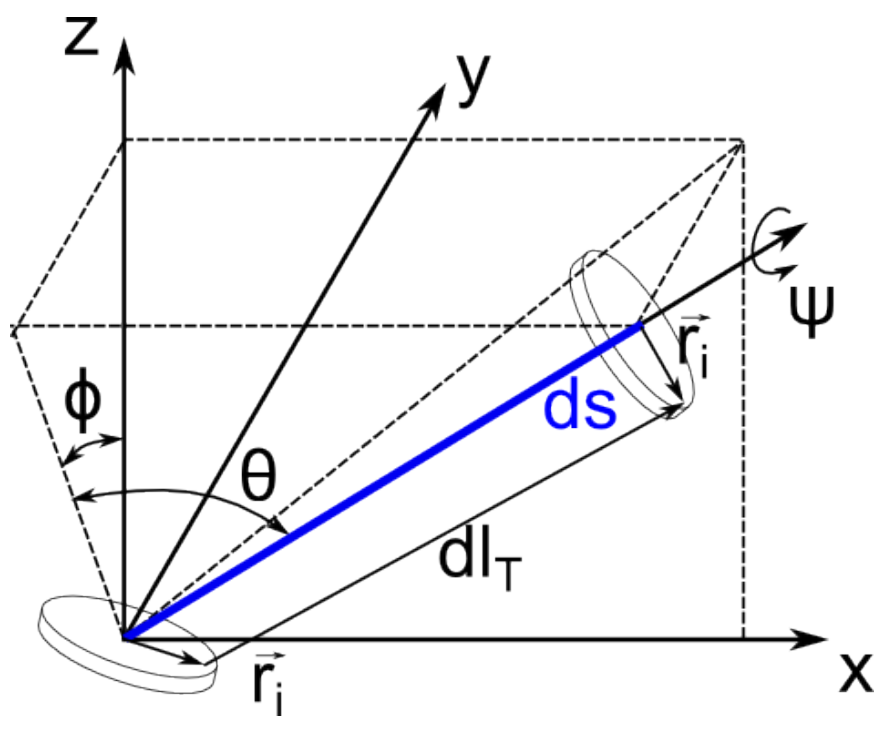

Figure 6.9: A differential three-dimensional segment of the manipulator with the backbone and tendon shown.

Further, from the beam mechanics block, using the three-dimensional model described in Section 3.3 .2 for a given position and orientation, the reaction loads at the distal end of the beam can be described by Eqs. 3.38 and 3.39 (repeated below in Eqs. 6.15 and 6.16). These results are then added in to the net load 
acting at the distal end of the manipulator.

$$
\begin{gathered}
F_{b, x}=\frac{\partial P E^{*}}{\partial x_{\text {end }}}, \quad F_{b, y}=\frac{\partial P E^{*}}{\partial y_{\text {end }}}, \quad F_{b, z}=\frac{\partial P E^{*}}{\partial z_{\text {end }}} \\
M_{b, \phi}(L)=\frac{\partial P E^{*}}{\partial \phi_{\text {end }}}, \quad M_{b, \theta}(L)=\frac{\partial P E^{*}}{\partial \theta_{\text {end }}}, \quad M_{b, \psi}(L)=\frac{\partial P E^{*}}{\partial \psi_{\text {end }}}
\end{gathered}
$$

Then using the relationships defined in Eqs. 6.17 and 6.10 in the system model (Fig. 6.8), the three dimensional position and orientation of the end mass can be computed from the translational and rotational inertial dynamics similar to the two dimensional case. A force and moment balance is applied to the distal end of the manipulator, treated as a free body as shown in Fig. 6.10. Loads applied to the end mass originate from the actuators, the reaction loads from the backbone, $F_{b}$ and $M_{b}$, and from any external loading, $F_{L}$ and $M_{L}$, as shown in Eqs. 6.17 and 6.18 . Force balances are with respect to the global reference frame while moment balances are with respect to the body frame described by the angles, $\phi(L), \theta(L)$, and $\psi(L)$.

$$
\begin{gathered}
\sum \vec{F}=m \ddot{\vec{x}}=\vec{F}_{b}+\vec{F}_{L}+\sum_{i=1}^{3} \vec{F}_{a, i} \\
\sum \vec{M}=\vec{I} \ddot{\vec{\phi}}+\dot{\vec{\phi}} \times(\vec{I} \dot{\vec{\phi}})=\vec{M}_{b}+\vec{M}_{L}+\sum_{i=1}^{3}\left(\vec{r}_{i} \times \vec{F}_{a, i}\right)
\end{gathered}
$$

where $\vec{I}$ contains the moments of inertia of the end effector about the local principle axes. The quantity $\vec{r}_{i} \times \vec{F}_{a, i}$ is the cross product between the position vector from the center of rotation to the point where the tendon terminates for each of the three tendons attached to the end mass as shown in Fig. 6.10 and the force vector applied at that point in the direction of the tendon in the deformed state and gives the contribution to the total moment due to each applied actuator load, $M_{a, i}$. Here it is again necessary to use the rotation matrix, Eq. 3.24, to operate on the position vector, $\vec{r}_{i}$, and the force vector, $\vec{F}_{a, i}$, for each of the actuators. Since 
the orientation of both of these vectors are known in the undeformed configuration, the rotation matrix can be used to represent these vectors in the deformed configuration. This in turn makes it possible to calculate the contribution to the net moments acting on the end mass by each of the actuators.

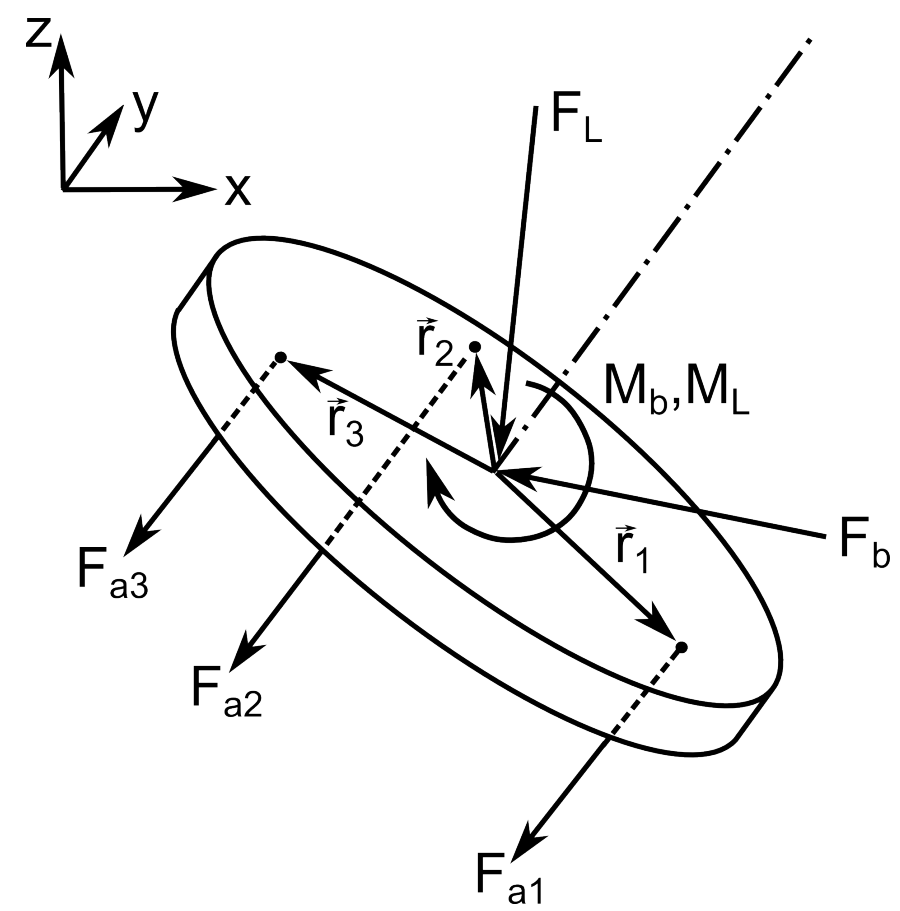

Figure 6.10: Free body diagram for the three-dimensional mass at the distal end of the manipulator.

\subsubsection{General Solution Method}

The general procedure for evaluating the system model as described in Sections 6.2 .1 and 6.2 .2 is the same whether considering the two-dimensional or threedimensional case. Using the analytical descriptions of each of the system components described in the preceding chapters, the state of the system at each time step can be evaluated as suggested in pseudocode below. The only steps which 
do not allow for closed form calculation are those involving the beam mechanics as the formulation for computing the beam loads and shape makes use of a computational minimization method as discussed in Sections 3.3.1 and 3.3.2.

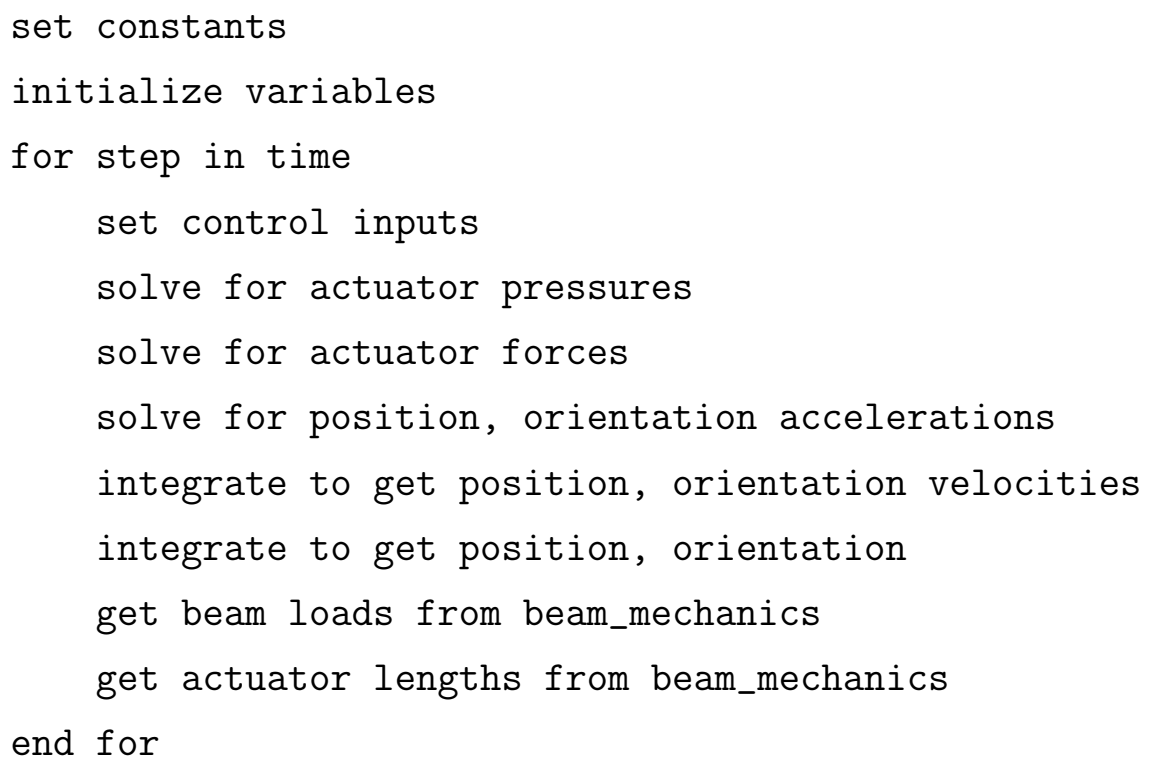

\subsection{Component Specifications}

The specific design of each of the system components requires consideration not only of overall constraints placed on the system described in Chapter 2, but also considerations unique to each individual component (Chapters 3, 4, and 5) and the interactions between each of the components that make up the assembly (Section 6.2. These values presented here describe the design scale characteristics for the minimally invasive surgical manipulator intended to meet the design requirements set forth in Chapter 2. While the requirements of any given surgical procedure may require modification to these specifications, the numbers set forth here should 
provide general guidelines for a capable robot that can be employed for a wide variety of abdominal procedures using transgastric access.

\subsubsection{Manipulator Backbone}

For the manipulator backbone, the size of the manipulator must be constrained to fit within the available tool space and thus the diameter of the routing disks located along its axis must have a diameter no greater than $6 \mathrm{~mm}$. Further, these disks should be spaced such that the manipulator can achieve maximum deflection. This is calculated using the ratio of disk radius to distance between disks where the ideal ratio occurs in the range of 0.2 to 0.5 as demonstrated by Li and Rahn, 2002 [87]. Thus, for a required maximum disk radius of $2.5 \mathrm{~mm}$ at the tendon routing location, the spacing between the disks should be $\in[5 \mathrm{~mm}, 12.5 \mathrm{~mm}]$ and most optimally at $6.25 \mathrm{~mm}$ [87].

Recall the two workspace specifications, an overall positional volume of $50 \mathrm{~mm}$ cubed and an angular range of $\pm 30^{\circ}$, described in Section 2.2. Figure 6.11 shows the working envelope for a two segment manipulator where each segment has a length of $50 \mathrm{~mm}$. The shaded region in each Sub-Fig. represents the achievable positions for an unloaded manipulator where the angular range of each segment is limited to $30^{\circ}, 60^{\circ}, 90^{\circ}, 120^{\circ}$, and $180^{\circ}$ for each Sub-Fig., respectively. This working envelope can be revolved around the longitudinal axis to project it into the third dimension. Assuming a two segment manipulator with a bend range of $180^{\circ}$, a manipulator segment length of approximately $50 \mathrm{~mm}$ would allow the device to reach the majority of the likely workspace, as shown in Fig. 6.11e. If the number of segments were increased, the length of each segment could be shortened.

Finally, the selection of a backbone material with a high capacity for strain would 


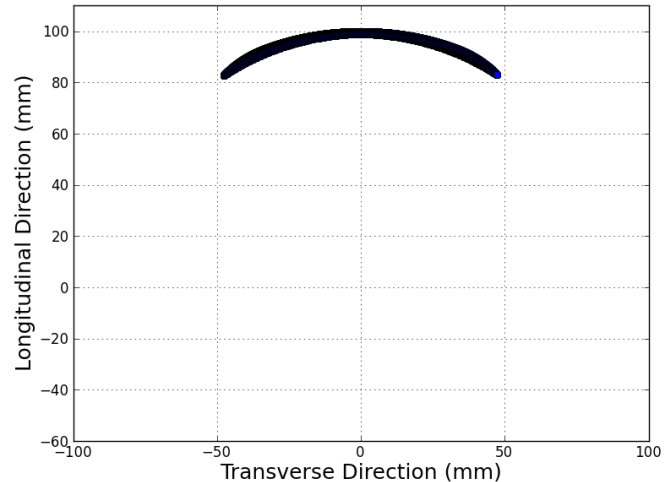

(a)

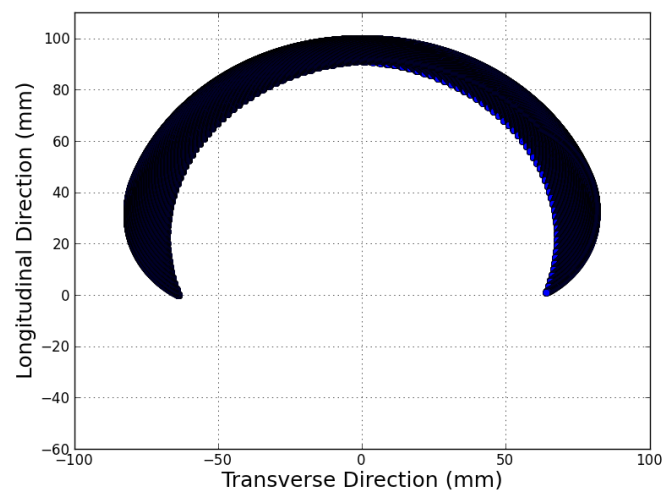

(c)

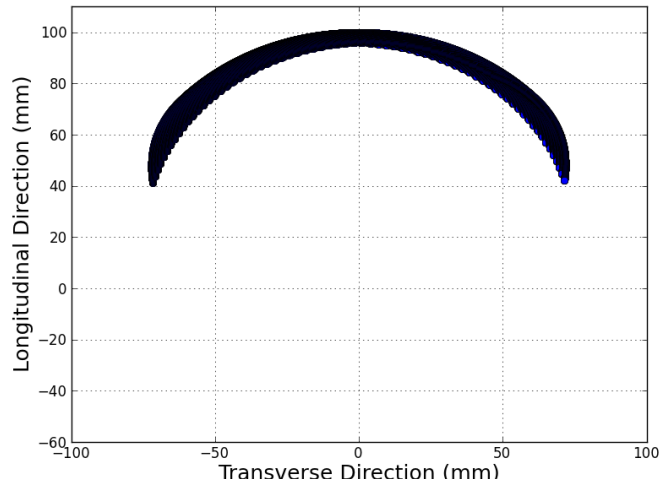

(b)

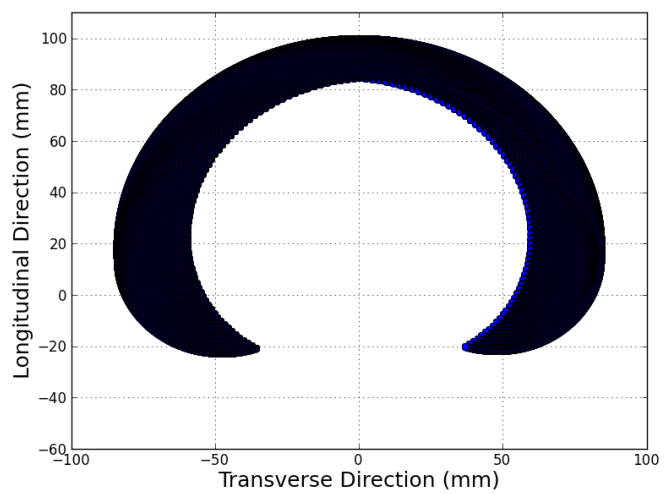

(d)

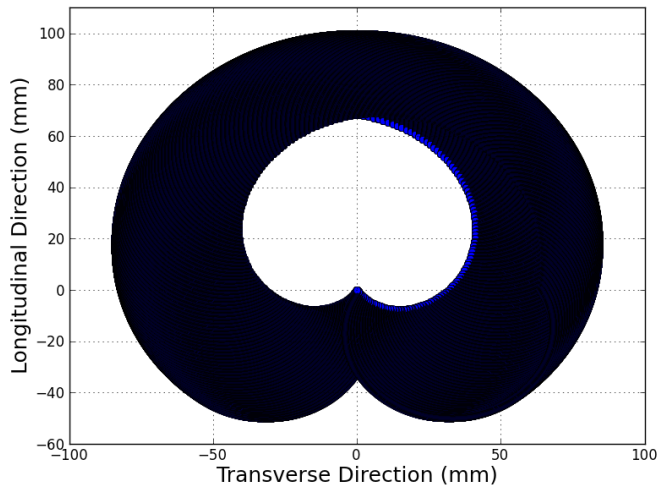

(e)

Figure 6.11: A set of five plots showing the planar working envelope of a two segment manipulator for a per segment bend range of (a) $30^{\circ}$, (b) $60^{\circ}$, (c) $90^{\circ}$, (d) $120^{\circ}$, and (e) $180^{\circ}$. 
aid in making the manipulator maneuverable without introducing permanent deformation. One attractive option is nickel titanium alloy, nitinol, which exhibits such characteristics. Also, it is desirable for the beam to be flexible in bending due to an applied load yet able to support itself in the unloaded state. For the scale of the manipulators discussed here, a diameter of approximately $0.5 \mathrm{~mm}$ is appropriate based on qualitative observations. For Martensite phase nitinol, the elastic modulus is on the order of $28 \mathrm{GPa}$ [123] compared to a similar material such as stainless steel for which the elastic modulus is on the order of $196 \mathrm{GPa}$ [161]. As such, the bending stiffness of the nitinol option is much lower than the stainless steel option.

\subsubsection{Actuation Unit}

For the specification of the artificial muscle actuator, it is assumed that the theoretical diameter limit, $D_{0}$, is $6 \mathrm{~mm}$. Further, the braid material is assumed to be nylon $\left(\sigma_{Y} \approx 50 \mathrm{MPa}\right)$ and the bladder is assumed to be made of latex rubber $\left(\sigma_{Y} \approx 3 M \mathrm{~Pa}\right)$ [132] with a wall thickness of $0.15 \mathrm{~mm}$ in order to maximize the available contraction range. Equation 6.3 gives the relationship between actuator length, $L_{a}$, actuator pressure, $P_{a}$, and contractile force, $F_{a}$. Thus two of these values must be specified to solve for the third. First, the desired condition of producing manipulator retroflexion will be used to design the actuator length.

To design the length of the actuator it is necessary to know the required muscle contraction, for which there is an achievable limit of approximately $32.5 \%$ of the elongated muscle length [125]. As shown in Eq. 6.7, the muscle contraction can be described as being equal to the value $r \cdot \theta(L)$ in the planar case. This means that for a single manipulator segment, manipulator bending of $180^{\circ}$ would require a length change during actuator contraction of $r \cdot \theta(L)=2.5 \mathrm{~mm} \cdot \pi=7.85 \mathrm{~mm}$. Due to the necessity for a given actuator to stretch by the same amount when the antagonistic actuator is activated, the total change in length between the 
extended and contracted states must be doubled. Thus assuming a contraction limit of $32.5 \%$, an actuator of length of $48.2 \mathrm{~mm}$ would meet this requirement.

Next, for the artificial muscle actuator, it is necessary to consider both the parameters of the braid and the length of the muscle. Applying the braid optimization methodology described in Section 4.3 at design scale and assuming a supply pressure of $700 \mathrm{kPa}$ suggests that the use of at least 30 strands and a minimum strand diameter of $0.14 \mathrm{~mm}$ yields the smallest braided mesh size while meeting the constraints for strand stress and bladder stress, assuming nylon wire is used for the mesh and latex rubber for the bladder. This corresponds to a theoretical minimum braid angle of $5.2^{\circ}$ and minimum diameter of $0.67 \mathrm{~mm}$. The real achievable minimum diameter is limited based on the deflated size of the inner bladder. From this value for $\gamma_{\text {min }}$, the necessary strand length, $b$, can be calculated from Eq. 4.15 as $48.4 \mathrm{~mm}$.

Using the specified design parameters yields a pick count of approximately 17 per inch (English units used to match braiding machine input). A sample mesh was produced using a braiding machine (Steeger HS 80/8VEA). A photograph of the machine braiding apparatus is shown in Fig. 6.12.

The mesh sample, shown in Fig. 6.13, demonstrates the braiding machine output with the braid parameters specifications suggested previously. The sample has a pick count of 15 which corresponds to an approximate edge length of $1 \mathrm{~mm}$ between each strand that crosses the long axis of the actuator.

Finally, it is necessary to consider the resistance of the antagonistic actuators and the bending resistance of the beam in the retroflexed state. The beam resistance is estimated using Eq. 3.3 and the antagonistic actuator resistance is calculated from the nominal pressure applied to the actuator in the open valve condition as well as the contribution from the elastic stretch using a spring constant of $0.07 \mathrm{~N} / \mathrm{mm}$, estimated from mechanical properties of general latex rubber and 


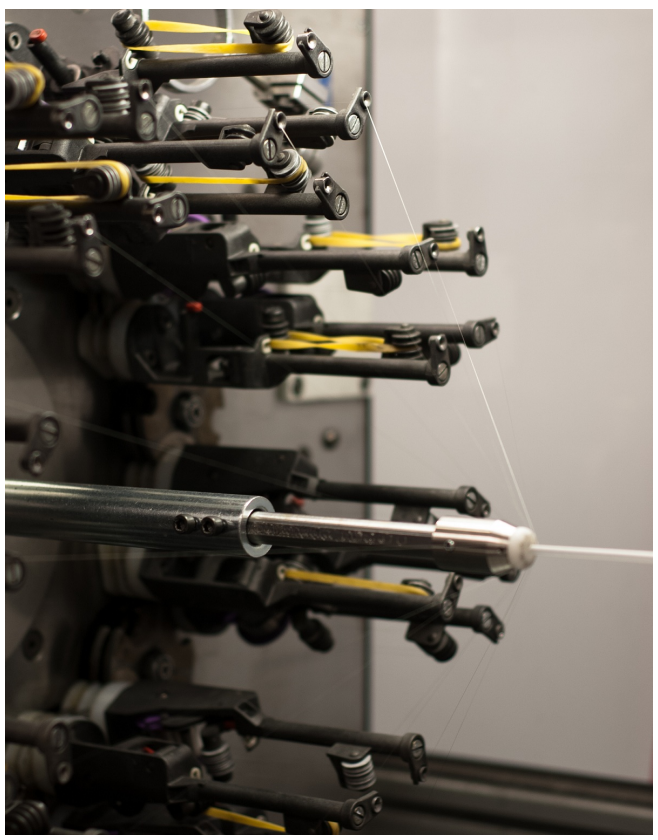

Figure 6.12: Photograph of a muscle actuator braiding process setup.

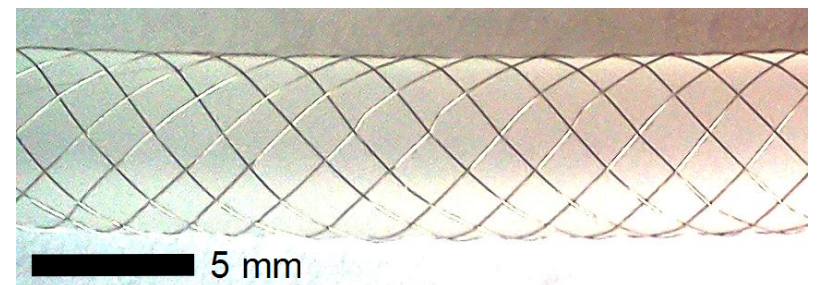

Figure 6.13: Photograph of braid sample at 15 pick per inch.

the geometry of the inner bladder [162]. Putting this all together results in an estimated actuator force requirement of approximately $5.68 \mathrm{~N}$ in order to achieve retroflexion of the planar manipulator. Going back to Eq. 6.3, it is possible to solve for the necessary actuator pressure under these conditions of approximately $575 \mathrm{kPa}$. It should be noted that these calculations were performed in the absence of load in order to establish a baseline for manipulator positioning. Either the pressure would need to be increased or the actuator would need to be longer in order to accommodate any load at retroflexion due to the relationship between actuator length and force capacity shown in Fig. 4.3. 
In terms of the load requirement of $5 \mathrm{~N}$ placed on the manipulator, the corresponding force requirement from the actuators is dependent upon manipulator tip position and orientation. For example, if the external load is applied in a direction parallel to the axis of the manipulator backbone at the distal end, then the force requirement from the actuator to begin moving the load would be the same magnitude as the load. Actuator forces greater than the load would result in a change in manipulator tip position and orientation until a new equilibrium position is found. Thus a $5 \mathrm{~N}$ load applied parallel to the manipulator backbone would require an actuator pressure of at least $100 \mathrm{kPa}$ to begin moving the load under the condition of full actuator extension. However, as the orientation of the manipulator changes, the pressure requirement would increase quickly due to contraction of the actuator and direction of the applied load with respect to the tip of the manipulator.

\subsubsection{Control Valve}

From the required actuator size, it is possible to determine the necessary design parameters for the control valve. For the actuator size given in the previous section, the volume change of the actuator would be on the order of $0.64 \mathrm{~mL}$, assuming the deflated volume is zero and the actuator inflates to approximately $5 \mathrm{~mm}$ in diameter in the contracted state. With the design goal of at most 0.5 seconds for full range of motion, a valve flow rate of $1.4 \frac{\mathrm{mL}}{\mathrm{s}}$ would be desired. Using Eq. 5.1 described in Section 5.3 and assuming a supply pressure of 700 $\mathrm{kPa}$ together with the necessary design average flow rate of $1.4 \frac{\mathrm{mL}}{\mathrm{s}}$ results in an upstream (fixed) orifice diameter of $0.4 \mathrm{~mm}$. To simulate the effects of reduced flow rate with increasing actuator pressure, the pressure was ramped to $350 \mathrm{kPa}$ (conservatively selected as an approximate upper bound on actuator pressure during high speed position changes) within a 0.5 second span and the total volume set to the design value above. Next, applying the principle of Equation 5.7 with 
an orifice ratio of 3 as discussed in Section 5.3 then results in a downstream orifice diameter $0.7 \mathrm{~mm}$ in the fully open condition (flapper nozzle behaves as though the standard orifice area is used in place of $A_{f}$ ). Using this diameter, the gap, $g$, between the orifice and the flapper is sized such that its nominal value falls under the fully open condition, here set to be $0.25 \mathrm{~mm}$.

For each valve design it is also necessary to consider the taper angle for the flapper. For the plug-based design, the taper angle of the plug is dictated by the geometric limitations of the valve body. Based on inspection of these geometric constraints the taper angle was set to $15^{\circ}$ as shown in Fig. 5.5. For the ring-based valve design, it is also necessary to consider the taper angle for the middle valve section as shown in Fig. 5.10, As previously mentioned, the higher the taper angle, the less the travel distance that is necessary for the ring to vary the manipulator stiffness. Based on the limits of the geometry a taper angle of approximately $10^{\circ}$ is the highest practical and results in a ring width requirement of at least 3.25 $\mathrm{mm}$. This geometry would be a challenge to fabricate. However, the taper angle could be reduced and the ring size increased to enable cheaper manufacturing.

\subsection{System Analysis}

\subsubsection{Theoretical System Analysis}

A model of the system was developed in MATLAB/SIMULINK ${ }^{\circledR}$ (The MathWorks Inc.) as a combination of the models described in the previous sections. From this model it was possible to theoretically evaluate the output of the system assembly under various input conditions.

First, the system response was modeled using the method of Section 6.2.1 for a two dimensional single segment manipulator in planar bending operated with a 
single active actuator by ramping the control input (variable orifice gap) from the open position to the closed position. The antagonistic valve orifice remains a constant flow restriction based on the relative orifice sizes since the orifice gap is large enough to not affect the flow restriction. Material and geometric properties are as stated in the previous section. In this case, the antagonistic actuator is in its deflated state and provides only resistance against the action of the active actuator due to elastic stretch of the internal bladder material. The result is shown in Fig. 6.14, where actuator pressure denotes the pressure at the feed point to the activated actuator, $P_{a}$, and tip orientation is the angular orientation of the distal tip of the manipulator. The tip orientation is zero when the manipulator is straight and has no deformation. At this nominal position, the actuators are in their undeformed state with lengths of $40.35 \mathrm{~mm}$. The plot shows that in the absence of external loading, the tip orientation approached $180^{\circ}$, corresponding to full retroflexion as specified in the design requirements, as the pressure at the actuator feed point approaches $570 \mathrm{kPa}$, consistent with the discussion in Section 6.3.2. In the fully retroflexed state, the active actuator has contracted to a length of $32.5 \mathrm{~mm}$ with an applied contraction force of $5.67 \mathrm{~N}$, while the antagonistic actuator has elongated to a length of $48.2 \mathrm{~mm}$ corresponding to an applied load of $4.26 \mathrm{~N}$. This result shows that the desired beam deflection can be achieved with approximately $83 \%$ of the supply pressure when no load is applied to the manipulator. However, the pressure to achieve retroflexion could be reduced by increasing the nominal length of the actuator. Further, since the primary contributor to the active actuator pressure requirement is the force applied by the antagonistic actuator, this requirement could be reduced by increasing the downstream orifice diameter such that the nominal pressure in the antagonistic actuator can reach a lower value. The simulation shown here was stopped once the angular orientation reached the design goal of $180^{\circ}$ as this point represents the design limit of motion based upon the elongated length of the actuators. It was found that the dynamics of this model was limited by the flow rate of the valve rather than the inertia of the manipulator due to low mass and low moment of 
inertia at the distal end, assumed to be on the order of $10.2 \mathrm{mg}$ and $16.2 \mathrm{mg} \cdot \mathrm{mm}^{2}$, respectively. The response shown here for the entire motion of the manipulator was completed in less than half of one second through the model prediction. This time was determined to be sufficient for most applications, especially true when considering that most applications will not require full retroflexion.

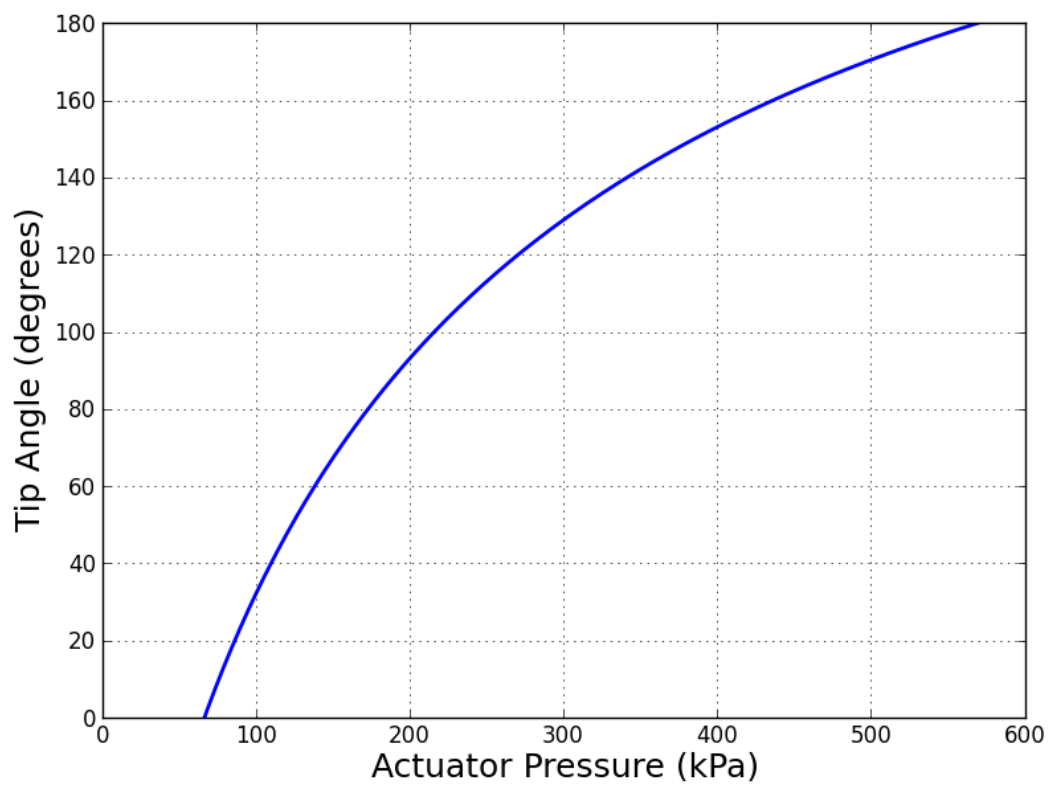

Figure 6.14: Plot of tool tip orientation versus actuator pressure for planar bending using the two dimensional model.

The range of motion for a single segment manipulator with no external loading is then defined by the sweep between the undeformed configuration and the fully deformed configuration as shown in Fig. 6.15. This sweep range from the planar approximation model can be rotated around the longitudinal axis of the undeformed manipulator in order to project the workspace into the third dimension.

Using the same analysis for the three-dimensional case where a single actuator is activated and two antagonistic actuators provide backside resistance by means of elastic stretch, it was found that the tip orientation (relative to bending in 


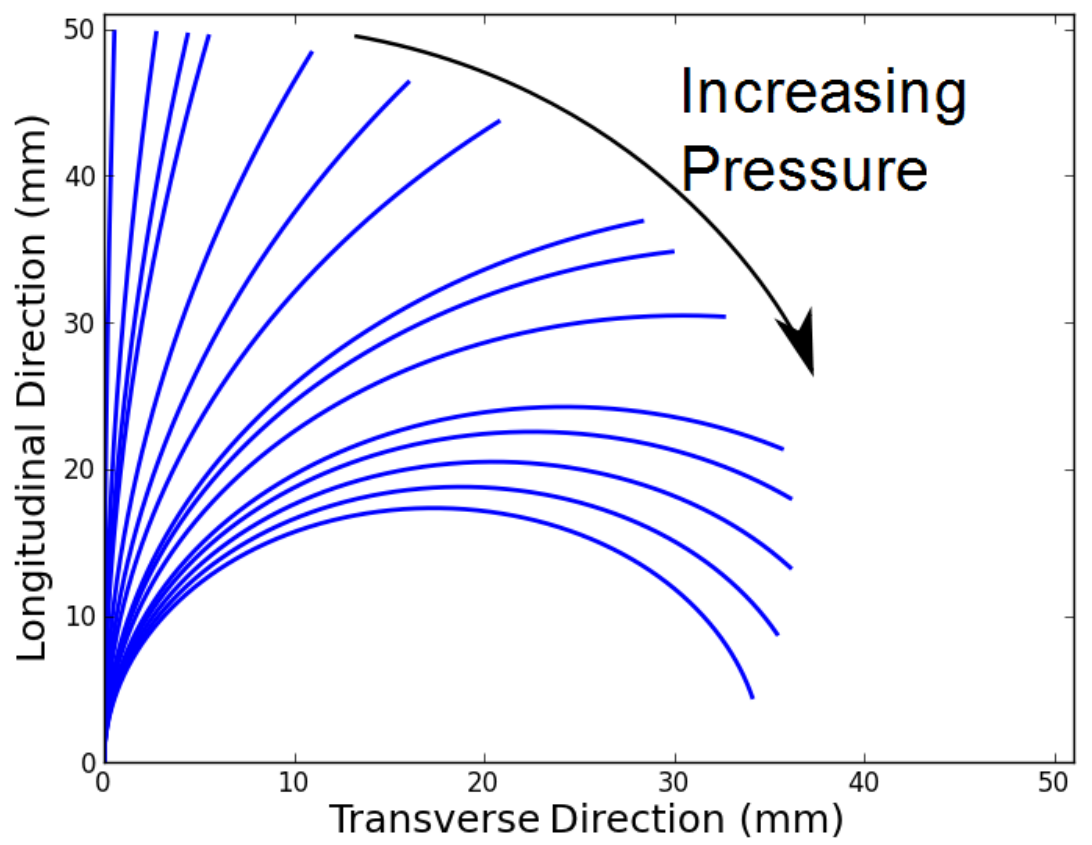

Figure 6.15: Plot of planar beam deflection for increasing actuator pressure with no external load for the two dimensional model.

the direction of the activated actuator) followed a similar curve as for the twodimensional case as shown in Fig. 6.16 but instead of making it to $180^{\circ}$ at 570 $\mathrm{kPa}$ the pressure required was only $455 \mathrm{kPa}$. This result is reasonable despite the fact that there are two antagonistic actuators since the termination points of the passive tendons are half the distance from the neutral axis when compared with the two-dimensional case as shown in Fig. 6.7. This means that the elongation of the passive actuators due to bending of the beam is reduced and thus the proportional antagonistic force would be slightly lower as well. The two passive actuators each elongate to a length of approximately $44.2 \mathrm{~mm}$ with applied loads of 3.11 N. Similarly, the active actuator contracts the same as in the two-dimensional model but with a contractile force of $4.52 \mathrm{~N}$.

It is also possible to increase the stiffness of the three actuators simultaneously through the use of out-of-plane positioning of the valve flapper as discussed in 


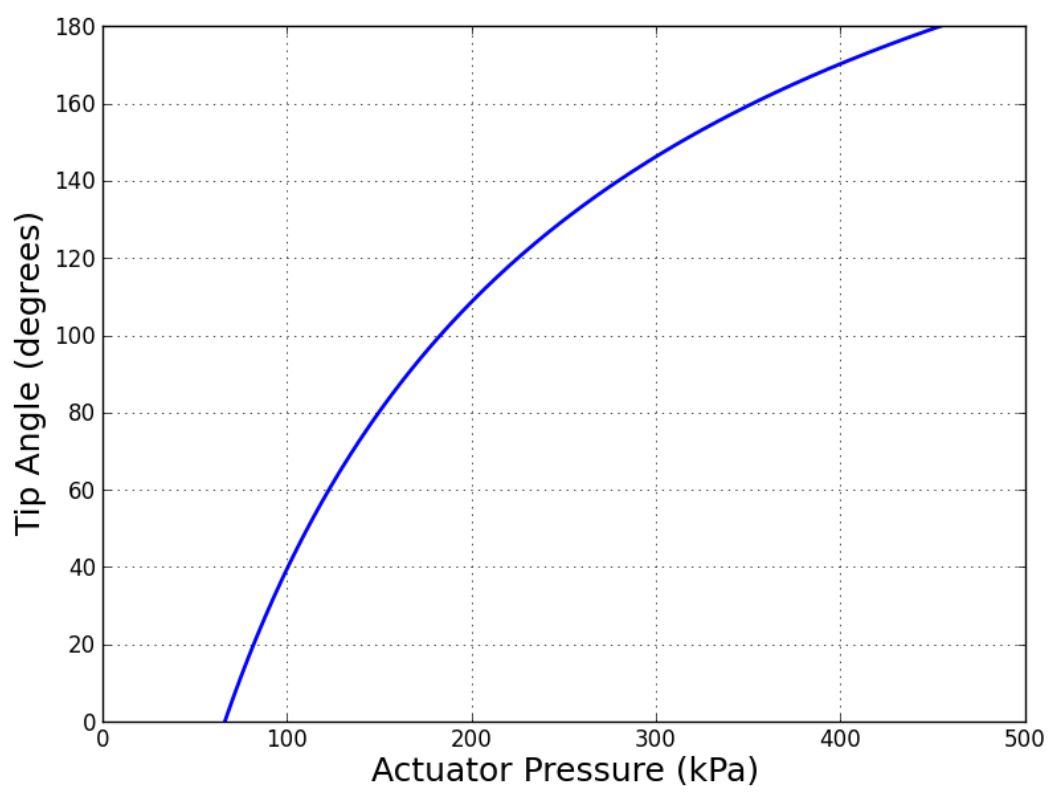

Figure 6.16: Plot of tool tip orientation versus actuator pressure for planar bending using the three dimensional model.

Section 5.5. This has the effect of increasing the nominal pressure in the actuators when the flapper is centered between the three downstream orifices. The flapper was initially positioned such that the nominal gap for each actuator was 0.18 $\mathrm{mm}$. The flapper was then moved towards the downstream orifice for a single actuator, as shown in Fig. 6.17, while simultaneously moving away from the downstream orifices corresponding to the two antagonistic actuators (see Fig. 6.18a). The rate of change of the flapper position was such that the tool tip orientation reached $180^{\circ}$ within one second as shown in Fig. 6.18b. This change in flapper position increases the pressure for one actuator while simultaneously decreasing the pressure for the two antagonistic actuators (Fig. 6.19a) which results in a contraction of the pressurized actuator and lengthening of each of the antagonistic actuators (Fig. 6.19b). Further, the pressure in the antagonistic actuators decreases until the corresponding gap size is large enough to have no effect on the flow restriction. At this point, the pressure applied to the actuator 
is dictated by the fixed diameters of the upstream and downstream orifices.

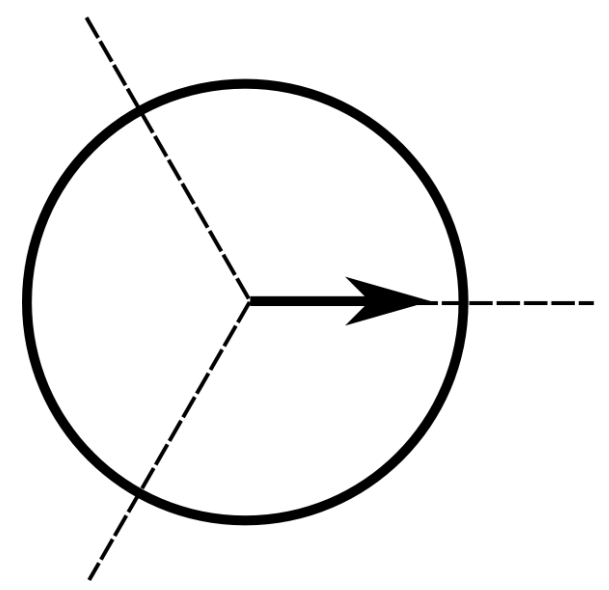

Figure 6.17: (a) Illustration of valve flapper in-plane position change where dashed lines correspond to orientation of each downstream orifice.

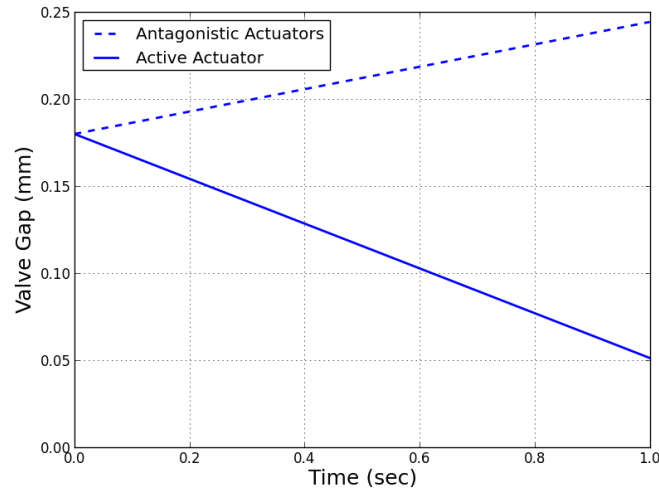

(a)

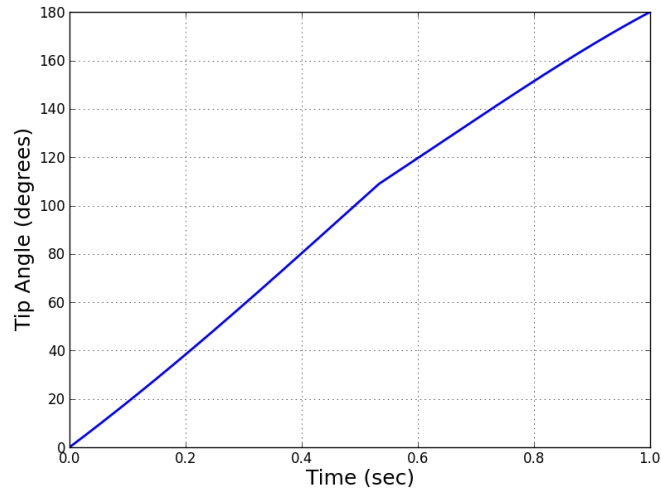

(b)

Figure 6.18: Plot of (a) gap size for both the active and antagonistic actuators and (b) tool tip orientation versus time.

Looking at the bending range in the three-dimensional case by individually activating each of the three actuators produces a similar result to Fig. 6.15 in that the beam deforms approximately along a circular arc in the direction of the activated actuator as shown in Fig. 6.20. The umbrella like shape of the curve defines the workspace of a manipulator segment in the unloaded case. 


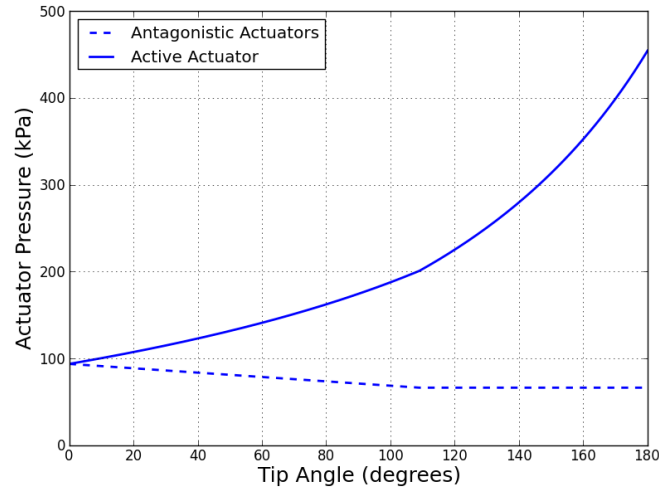

(a)

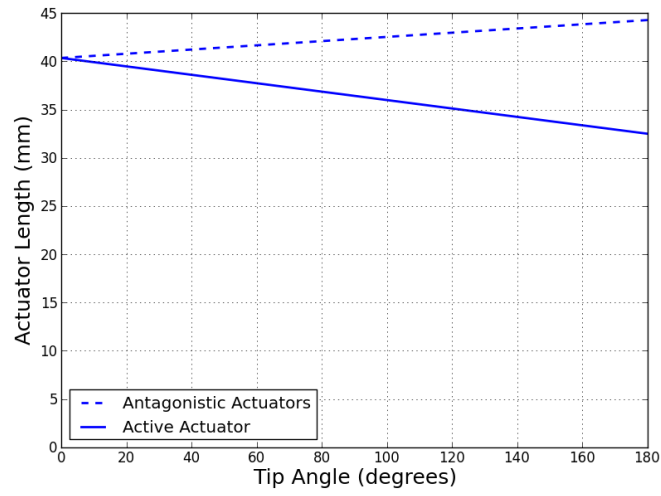

(b)

Figure 6.19: Plot of (a) actuator pressure and (b) actuator length versus tool tip orientation for simulation with increased actuator stiffness.

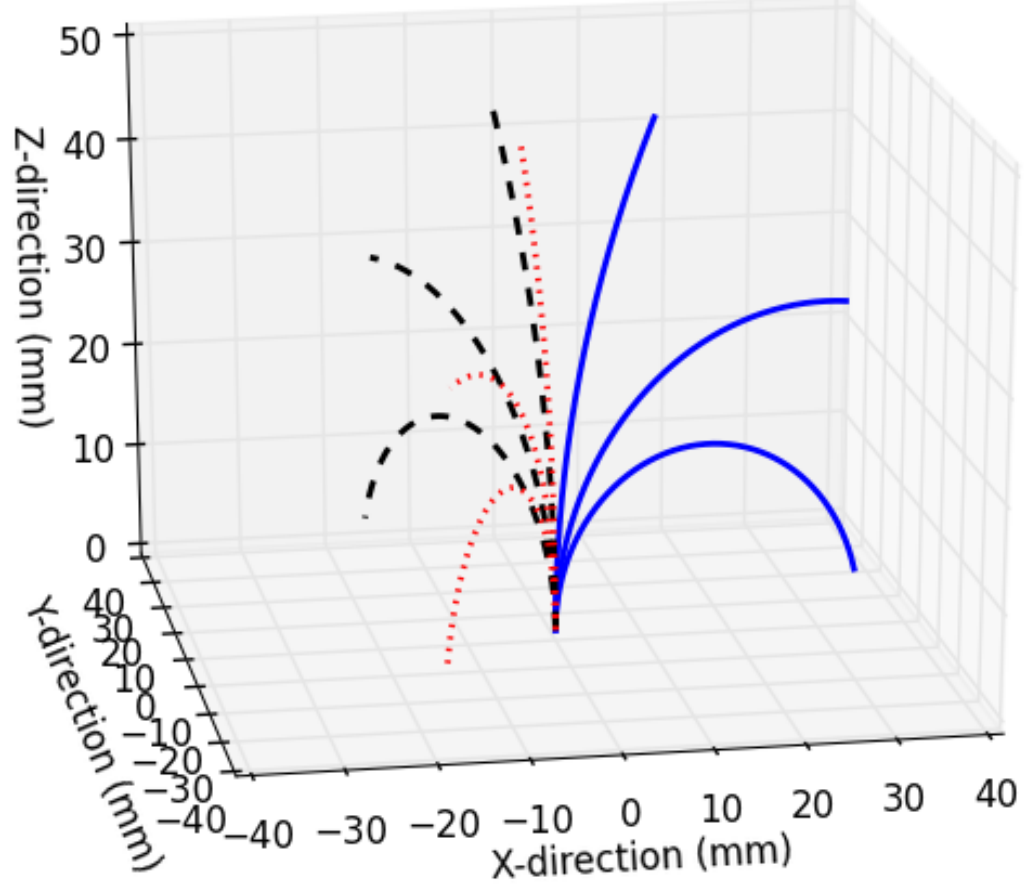

Figure 6.20: Plot of beam deflection for varying actuator pressures applied to each of the three actuators with no external load. 
Activating an additional actuator has the effect of revolving the manipulator about its long axis by changing the resultant direction of the applied moment. An example of this is shown in Fig. 6.21. Here the pressure applied to each actuator was ramped towards $300 \mathrm{kPa}$ with a small time delay between the activation of the first and second actuators, as shown in Fig. 6.22. The result shows that the manipulator deformed in planar bending as before prior to the activation of the second actuator. Upon activation of the second actuator, the manipulator exhibited out of plane deformation about the long axis with increasing magnitude as the pressure in the second actuator increased. At the end of the simulation, the orientation of the manipulator tip measured relative to the positive z-axis was approximately $187^{\circ}$, slightly greater than the retroflexion condition. This was achieved at a much lower pressure than was calculated under previously simulated conditions using a single active actuator due to the use of two actuators to achieve manipulator bending.

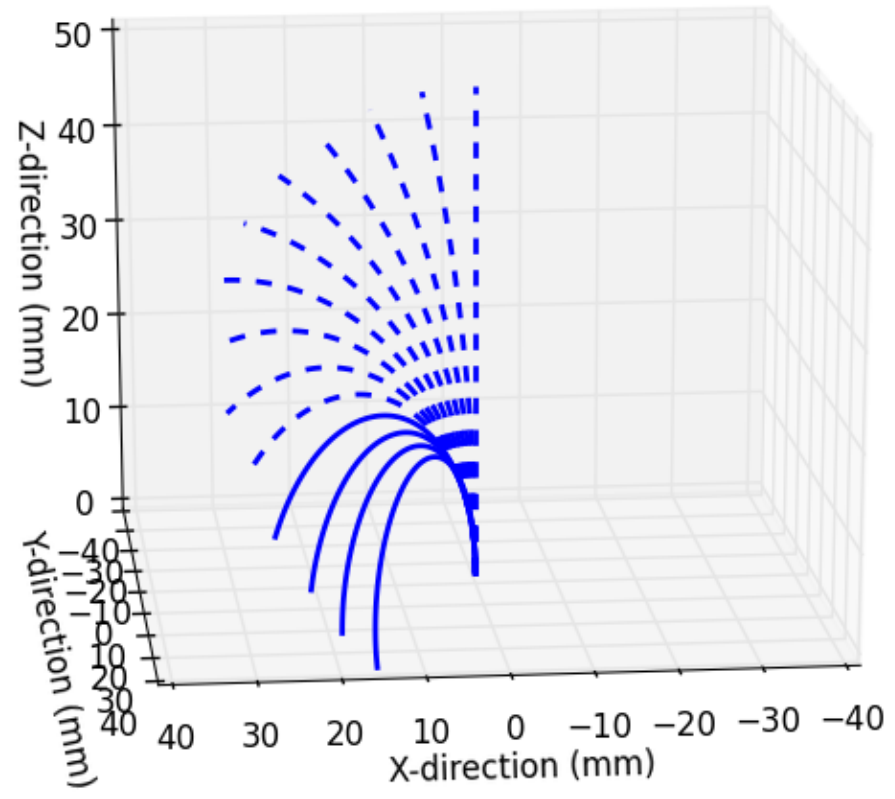

Figure 6.21: Simulation of three dimensional beam deflection through activation of two actuators. 


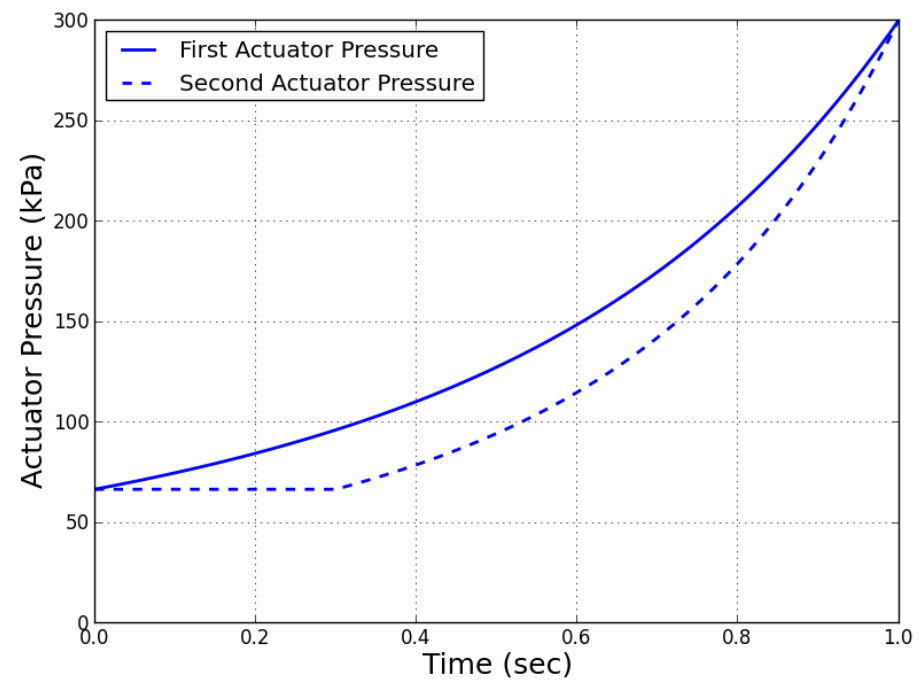

Figure 6.22: Actuator pressures for each of the activated actuators.

The application of external load on the end of the manipulator can produce interesting results as the beam shape and the load on the end effector can change depending on current beam configuration and direction of the applied load. An example analysis of the effects of external load is shown in Fig. 6.23. First, the manipulator was deformed to the configuration shown by the dashed line through the application of $270 \mathrm{kPa}$ to a single actuator. Next, a $0.5 \mathrm{~N}$ load was applied in the positive longitudinal direction as indicated in the figure. It is interesting to note that the beam tip not only deflects in the longitudinal direction due to the applied load but also shifts in the negative transverse direction as the beam adjusts to a new, stable configuration. This is a result of the unactuated manipulator degrees of freedom which passively determine the beam shape when the manipulator is subject to a load. A second similar analysis was performed in which a load was first applied to the beam with no actuator inflation followed by an increase in actuator pressure, the results of which are shown in Fig. 6.24. An external load of $5 \mathrm{~N}$ was applied in the negative transverse direction causing the beam to deflect to a fully extended configuration as shown by the dashed line. Then the pressure in the actuator which contracts the right side of the beam was increased to 700 
$\mathrm{kPa}$. This resulted in a recovery of some of the initial deflection experienced by the beam, moving the load approximately 20e mm back in the positive transverse direction, indicating the ability to move a load of this magnitude.

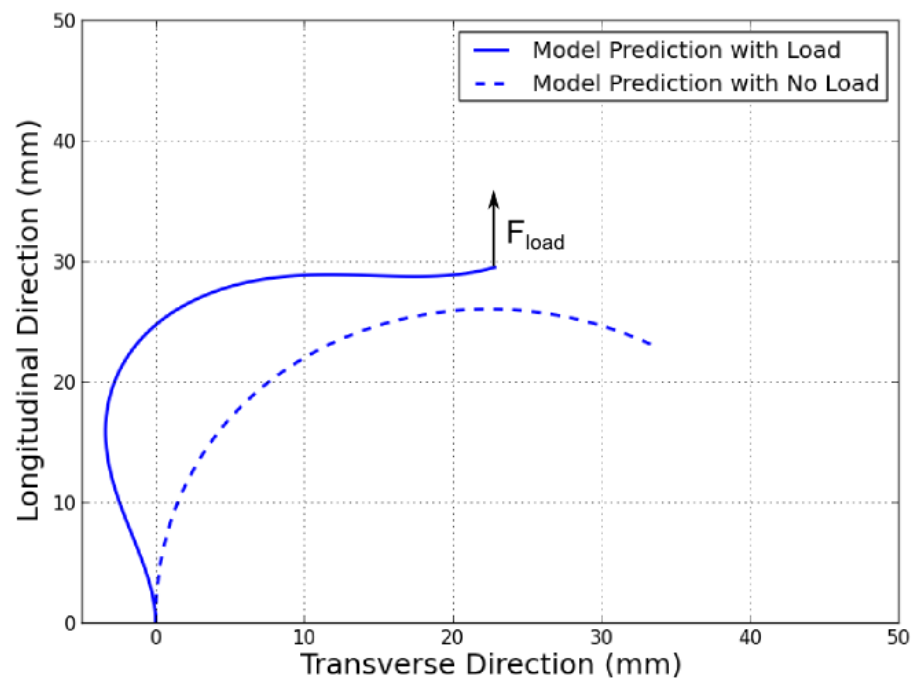

Figure 6.23: Calculated beam shape for a beam deflected by a single actuator with and without an end load applied.

\subsubsection{Computer Rendered System Assembly}

Using the dimensions of the design scale system, a computer rendering was produced to give a visual of how the components could be assembled together (full model available at [163]). Contained within the body of the endoscope are the valves (plug-based design) and actuators necessary to operate the manipulators, as shown in Fig. 6.25. The actuators for each manipulator segment are arranged in series to maximize the available space within the endoscope working channel. Arranging the actuators in series requires that fluid transfer lines be used to distribute the flow of fluid from the valve to each actuator. Additionally, retainers are used to provide a fixed plate to which the actuators can be anchored such that AMA contraction created through pressurization of the actuator is applied to the 


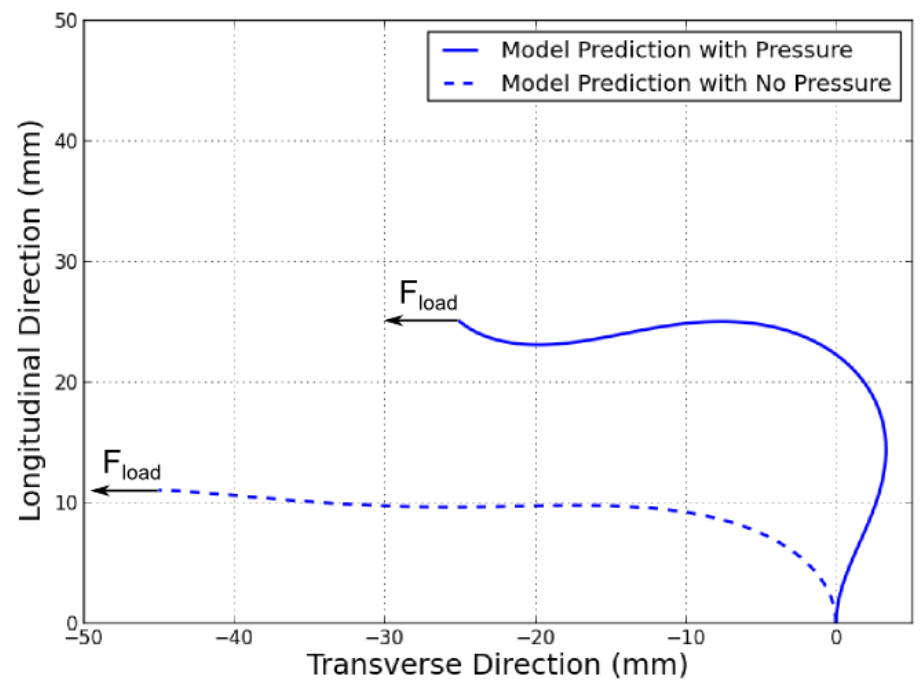

Figure 6.24: Calculated beam shape for a beam deflected by an end load with and without a pressure applied to the actuator.

connected tendon. Each retainer includes slots to allow the anchored end of the actuator to change position as it inflates. This change in position also requires that the fluid transfer lines be flexible such that they can move with the actuators. The retainer slots also provide passage for the fluid transfer lines for distally located actuators. An example of the working channel cross-section is shown in Fig. 6.26 where a single actuator is shown in its fully inflated state alongside two deflated actuators. The fact that the fully inflated actuator does not occupy the full working channel diameter allows enough remaining space for the fluid transfer lines as well as channels for tendon routing without interference.

The valves are connected to the existing suction and irrigation lines within the endoscope (see Fig. 6.27) to eliminate the space requirement for the extra hydraulic lines. Putting it all together, two, two-segment manipulators might maneuver within the defined workspace as shown in Fig. 6.28. In this figure, an endoscope is shown with two tool channels; each containing the components for two, two-segment manipulators. The components for each manipulator segment are 


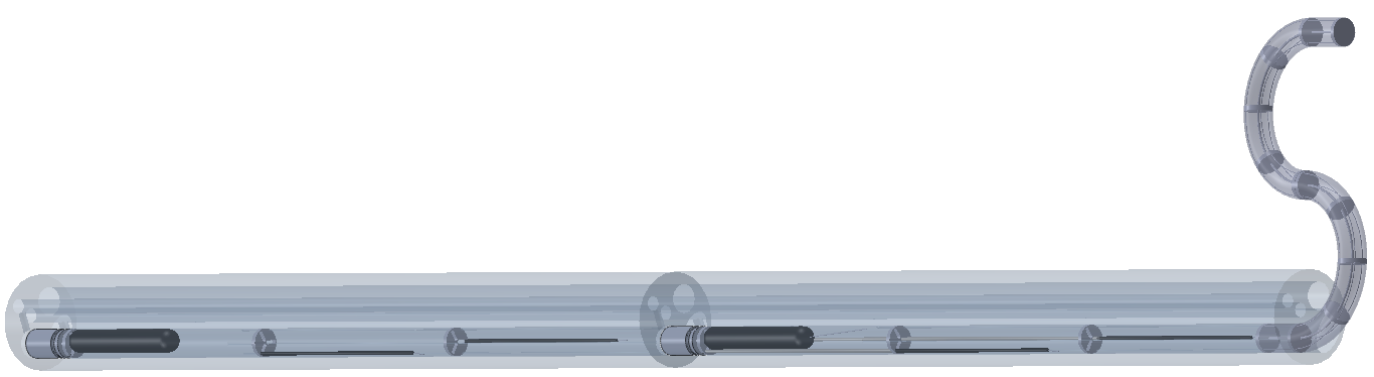

(a)

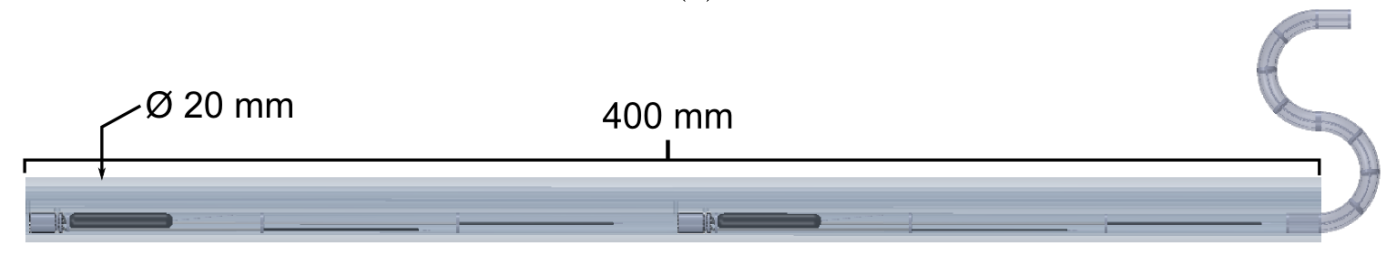

(b)

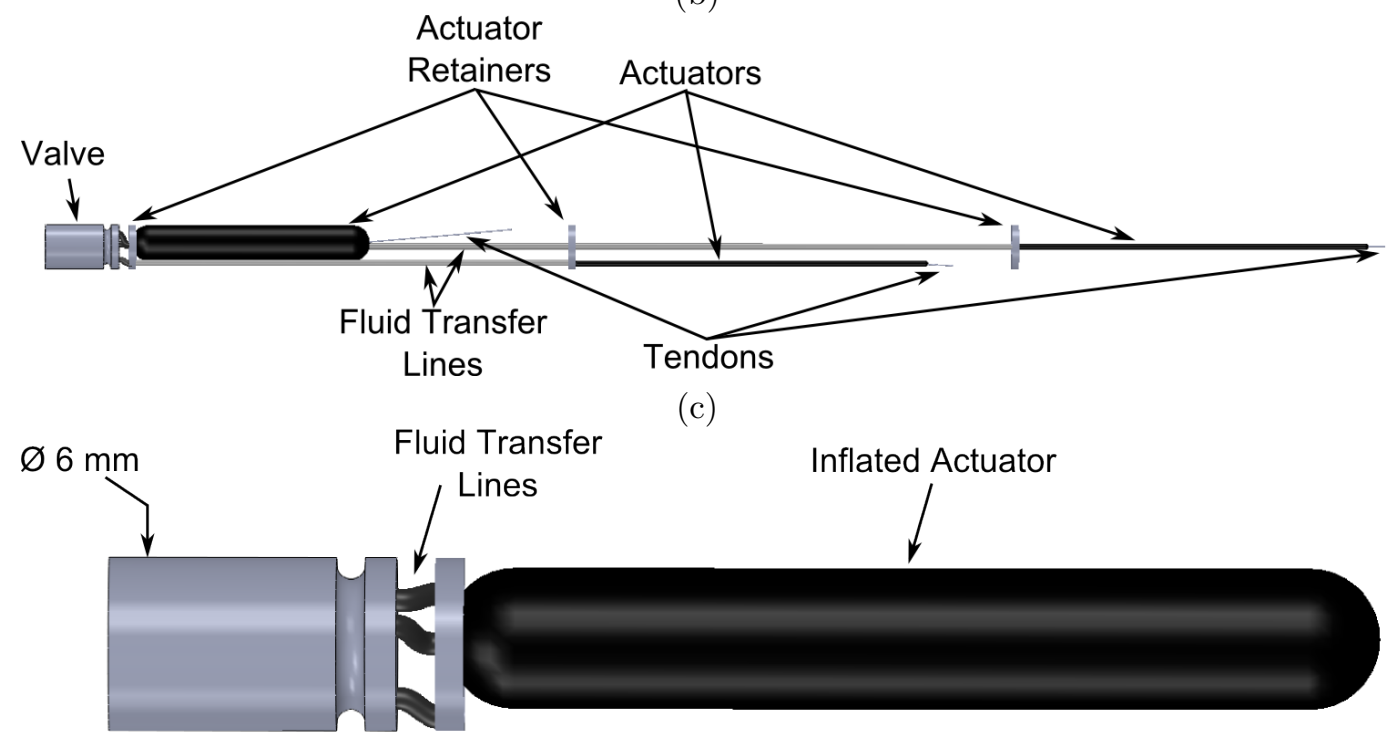

(d)

Figure 6.25: Computer rendering showing the full manipulator assembly in (a) perspective view and (b) side view, (c) the internal components used to operate a single segment of one manipulator, and (d) the connection between the valve and a single actuator shown in its inflated state. 


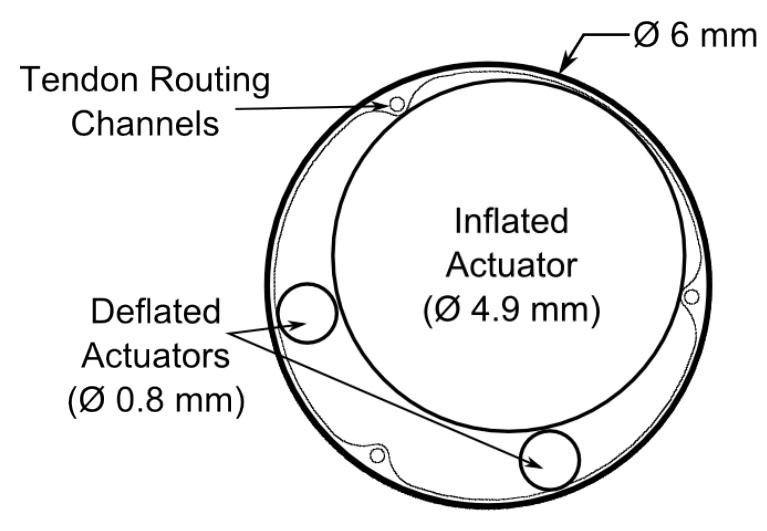

Figure 6.26: Illustration of the cross section of the working channel of an endoscope showing three actuators arranged in parallel, one of which is fully inflated and the routing channels for the manipulator tendons.

arranged serially within the working channel and the tendons for the proximal segment are routed along the outer radius of the working channel as shown in Fig. 6.26 in order to avoid interference with the components for the distal segment.

\subsubsection{System Prototyping and Testing}

Limitations due to the valve not performing as intended as discussed in Chapter 5 prevented the hydraulic surgical robot system from being assembled in a controlled fashion. However, it was possible to test the interaction between the artificial muscle actuator and the continuum beam manipulator by manually inflating the actuator using a pressure instrumented syringe (BARD Caliber Inflation Device) as shown in Fig. 6.29 or through manual manipulation of the ring-based valve. This made it possible to get a better understanding of the behavior of the actuator and manipulator in response to changing pressure. For this testing a prototype of a manipulator backbone with attached actuators was produced from available 


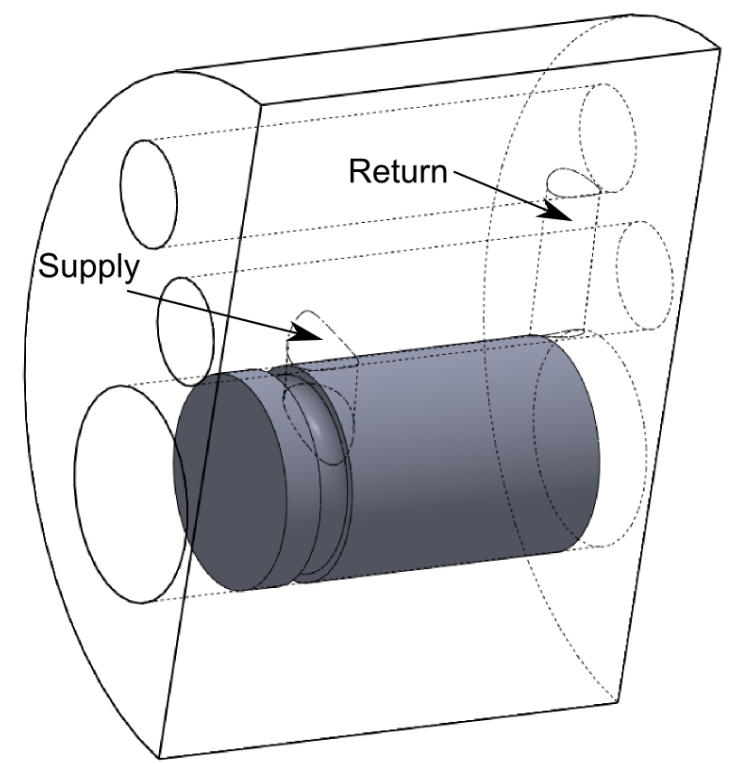

Figure 6.27: Illustration of the valve within the working channel of an endoscope with channels providing connection to the suction and irrigation lines.

materials. The backbone was made from a length of nitinol wire with a diameter of $1 \mathrm{~mm}\left(E I=1.37 \cdot 10^{-3} \mathrm{~N} \cdot \mathrm{m}^{2}\right)$ and a length of $185 \mathrm{~mm}$. The disks used to route the tendons had a radius of $6 \mathrm{~mm}$ at the location of the tendon.

As it was not possible to produce a muscle actuator at design scale, a version of the muscle actuator was produced using latex surgical tubing as the internal bladder and pre-manufactured nylon mesh, with an inflated outer diameter of 8.8 $\mathrm{mm}$, as shown in Fig. 4.10 and described in Section 4.4. This muscle actuator was found to perform as predicted analytically in terms of contraction range as demonstrated in Section 4.4. For this to be possible, care was taken to coordinate the elongation of the outer mesh with the deflated length of the surgical tubing. In particular, it is important to ensure that minimal clearance exists between the outer mesh and the bladder in order to prevent the bladder from crossing an elastic instability point (aneurysm formation) [164]. To do this, it is necessary to stretch the inner bladder and outer sheath together. It is also necessary to ensure 


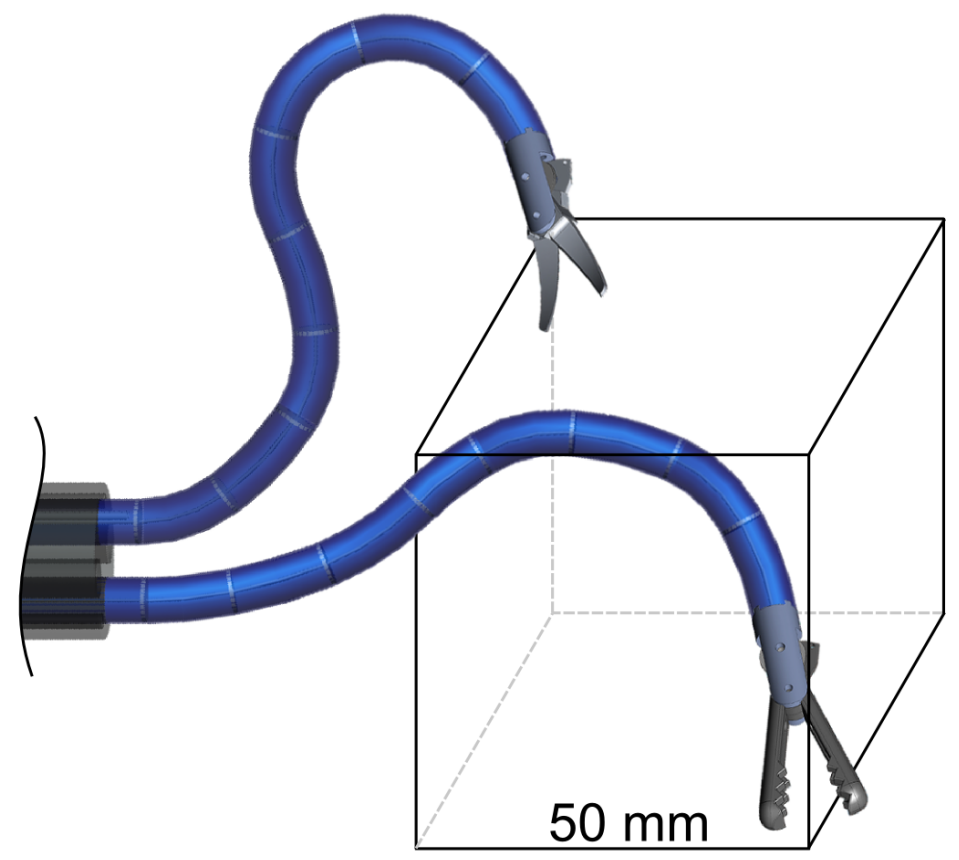

Figure 6.28: Computer model of design scale, two segment per manipulator system with workspace shown. Shown with example end effector tools including a grasper and a scissors 84].

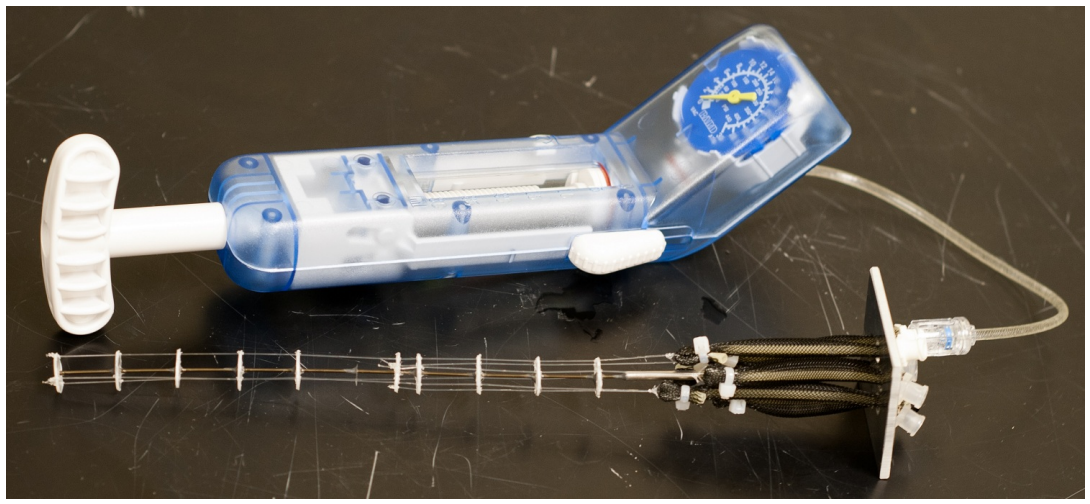

Figure 6.29: Photograph of a pressure regulated syringe and manipulator testing apparatus. 
that the actuator is not taut when connecting it to the tendons thus allowing the actuator to elongate when an antagonistic actuator is activated. In practice this has the effect of slightly increasing the pressure necessary to inflate the bladder due to an increased level of wall stress that must be overcome. To minimize this effect it is ideal to select a bladder material with high elasticity (compliance $>0.05$ $\left.\frac{m^{2}}{N}\right)$.

In the planar case with a pressure up to $1.03 \mathrm{MPa}$ applied to only a single actuator using the instrumented syringe, the actuator and manipulator were found to perform as predicted by the model. The manipulator behaves as in the planar case presented in Section 3.3 , exhibiting no out-of-plane deformation and reproducing a circular arc for which the radius of curvature decreases with increasing actuator pressure. This result is expected as the inactive actuators provide symmetric opposing forces on the antagonistic side of the manipulator. The forces from these actuators reduce the output from the active actuator (but not significantly) and actually improved the lateral stability of the manipulator. A photograph of the manipulator under these conditions is shown in Fig. 6.30. The pressure required to achieve full retroflexion was recorded as approximately $280 \mathrm{kPa}$. This pressure is subject to variability according to the nominal actuator length, actuator construction parameters such as amount of pretension, and geometry of the manipulator. When an additional actuator was activated, the curvature of the manipulator revolved around the longitudinal axis to the new equilibrium orientation. In the absence of external loading, the manipulator shape remains planar as predicted by the model.

It was also possible to evaluate system performance through manual manipulation of the ring-based valve prototype. Because the actuator used here was larger than the design scale, it was necessary to increase the size of the ring-based valve orifices to accommodate the higher flow rate requirement of $5.0 \frac{\mathrm{mL}}{\mathrm{s}}$. Using the same procedures described in Section 5.3, an upstream orifice diameter of $0.74 \mathrm{~mm}$ was necessary. Equation 5.7 then results in a downstream orifice of diameter 1.3 


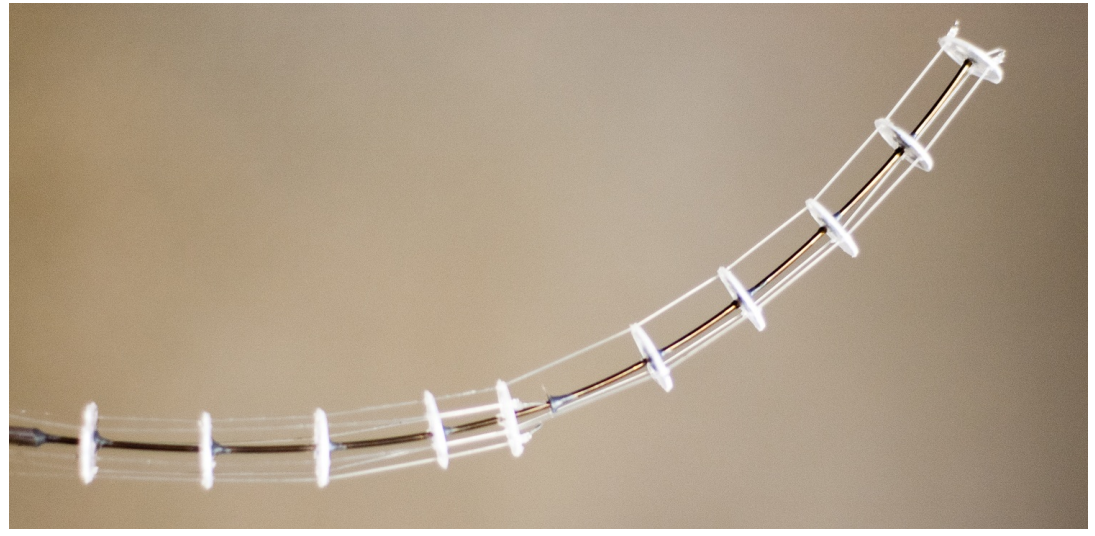

Figure 6.30: Photograph of manipulator backbone under load.

$\mathrm{mm}$ in the fully open condition. Using this diameter the necessary gap, $g$, between the orifice and the flapper under the fully open condition is $0.4 \mathrm{~mm}$. These valve orifices for both the upstream and downstream orifices are sized to achieve both sufficient flow rate and appropriate valve control for this valve prototype. Also, for this prototype, a taper angle of $3^{\circ}$ was used in order to make manual manipulation of the valve ring possible.

The outlets of the ring based valve design were connected to the same muscle actuators and pressure was supplied using the same pump setup as shown in Fig. 5.15 with a constant supply pressure of $689 \mathrm{kPa}$. To test the interaction between the ring-based valve design and the actuators, the ring flapper was manually manipulated to differentially open and close the flapper nozzles. When one of the flapper nozzles was fully blocked by the ring, the actuator was inflated in 0.35 seconds on average. This confirmed the design goal of 0.5 seconds for full actuator contraction which was used to size the orifices. Further, it was found that two actuators could be inflated by positioning the near side of the ring between the two corresponding nozzles. Finally, by adjusting the out-of-plane position of the ring it was possible to adjust the relative neutral state between the three actuators (stiffness control). 
Being able to vary the stiffness of the actuators as a unit provides some measure of control over the baseline rigidity of the manipulator as deflection in any direction requires more load to overcome the actuator loads. To get an idea of what the effect of this variable stiffness is, the actuators were inflated simultaneously while using a pressure gage. A lateral load was then applied to the manipulator using a spring scale and the deflection of the manipulator was measured as shown in Fig. 6.31. The test was carried out at two positions along the length of the beam. Position 1 was located $60 \mathrm{~mm}$ from the fixed base and Position 2 was located 185 $\mathrm{mm}$ from the fixed base. The results of this test are shown in Table 6.1.

Table 6.1: Results from manipulator stiffness measurements.

\begin{tabular}{ccc}
\hline $\begin{array}{c}\text { Inflation } \\
\text { Pressure }\end{array}$ & $\begin{array}{c}\text { Load Position 1 } \\
\text { Stiffness }\left(\frac{N}{m m}\right)\end{array}$ & $\begin{array}{c}\text { Load Position 2 } \\
\text { Stiffness }\left(\frac{N}{m m}\right)\end{array}$ \\
\hline $0 \mathrm{kPa}$ & 0.08 & 0.014 \\
$345 \mathrm{kPa}$ & 0.12 & 0.018 \\
$689 \mathrm{kPa}$ & 0.19 & 0.024 \\
\hline
\end{tabular}

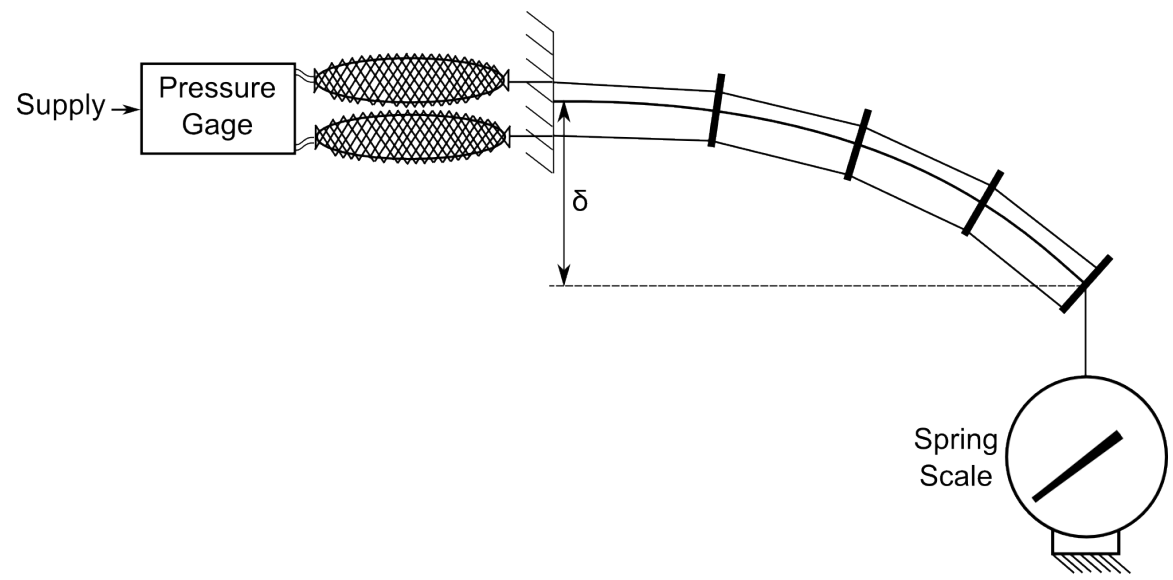

Figure 6.31: Illustration of test setup for measurement of manipulator stiffness. 


\subsection{Summary of System Assembly}

The system model described here incorporates each of the individual components that make up the hydraulically powered surgical robot design. The model has been formulated in both two-dimensional and three-dimensional versions. The two-dimensional version allows for simulation of the assembled surgical manipulator subject to planar deformation and is suitable to simulate manipulator motion when no out of plane external loading is applied. The model includes the analytical descriptions of each of the robotic components described in previous sections. Where necessary, these components are coupled together through the relevant hydraulic and geometric relationships. The two-dimensional model was able to demonstrate that the design goal of 0.5 seconds to achieve full retroflexion is possible with the included valve design. The simulation suggests the ability to manipulate a load of $5 \mathrm{~N}$. However, the magnitude of the distance that this

load could be moved is not large. This result may indicate the need to explore higher actuator pressures to achieve greater capability depending on the specific intended task of the manipulator. Further, at higher operating pressures it would be possible to reduce the size of the actuators thus making parallel arrangement of the actuators feasible. This would in turn reduce the overall length of the manipulator components within the endoscope as shown in Fig. 6.32. For example, increasing the operating pressure to $3.5 \mathrm{MPa}$ would allow the actuator diameter to be reduced to a theoretical value of $1 \mathrm{~mm}$ to achieve the same load capacity. However, the increased pressure would necessitate a denser braid which would reduce the contraction range and thus increase the required length of the actuator. Further, the use of higher operating pressures and higher actuator loads could be combined with a stiffer manipulator backbone in order to reduce transverse deflection due to external load. Fortunately, the loads common to many surgical tasks are significantly smaller and thus more achievable. As compared against similar surgical robots presented in the literature, the system models suggest that appropriate criteria for robotic manipulation speed and load capacity can be achieved 
without the use of external actuators or motors.

One challenge with the system model is computational effort required to compute the output. For both the two-dimensional and three-dimensional system models, the use of an optimization algorithm for modeling the beam response prevented real-time operation of the model. Pre-computation of the beam output for a variety of end loads was found to improve computation time but not enough to permit real-time simulation. Further enhancement of the system model will allow this simulation to be used as a plant model for system controller design.

Testing of the system using manual inflation with an instrumented syringe confirmed the model predictions for beam shape achieved during actuator contraction. Further, in the absence of external load, the manipulator exhibited planar deformation which is also consistent with the model prediction. When the actuator and backbone prototype was connected to the ring based flapper valve, it was possible to measure the contraction time of an actuator. This result confirmed the performance of the valve prototype which was designed to achieve full actuator contraction within a maximum of 0.5 seconds. Further, by simultaneously inflating each of the three actuators which are used to operate the prototype segment, it was possible to get an idea of the effect of baseline actuator pressure on manipulator stiffness. These results indicated that greater pressures do indeed result in greater stiffness. This situation is useful in the case of increasing manipulator stability during a given task. However, it comes at the expense of load manipulation ability as the actuator acting in the direction of intended motion would have to act against the antagonistic actuators in addition to the load itself. 


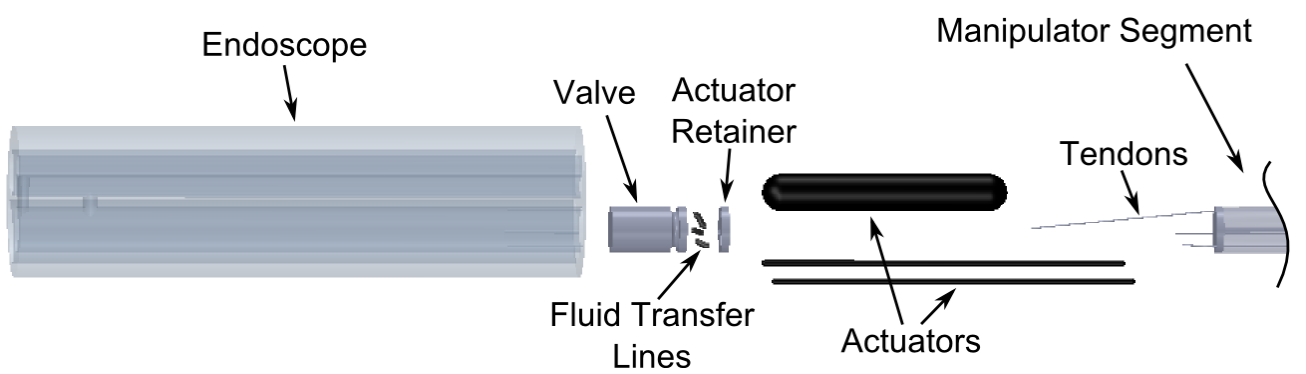

(a)

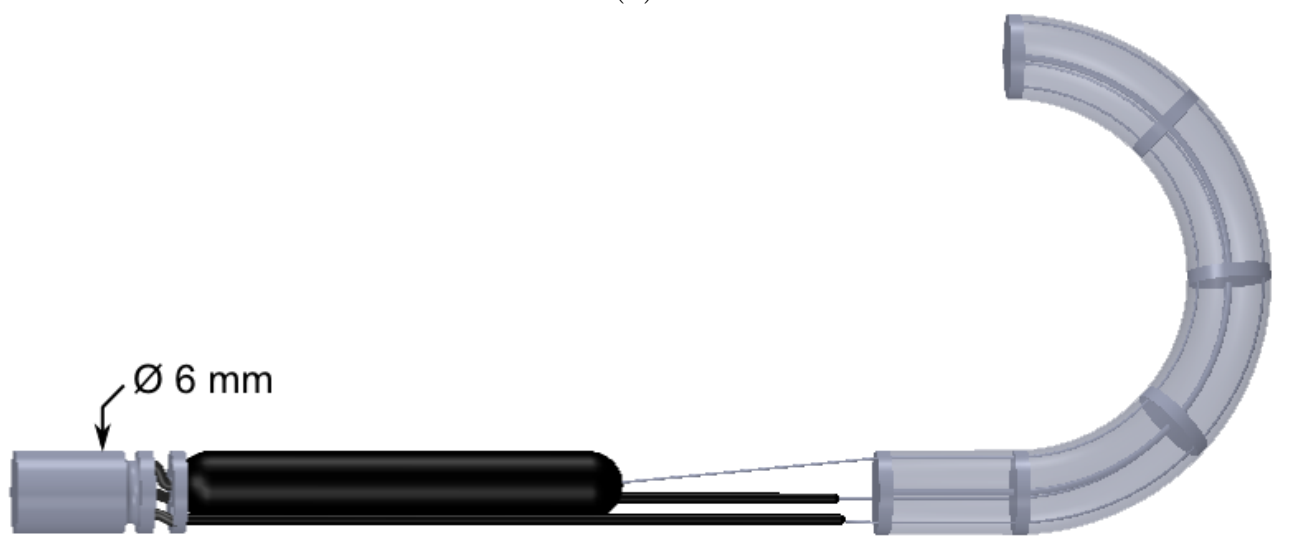

(b)

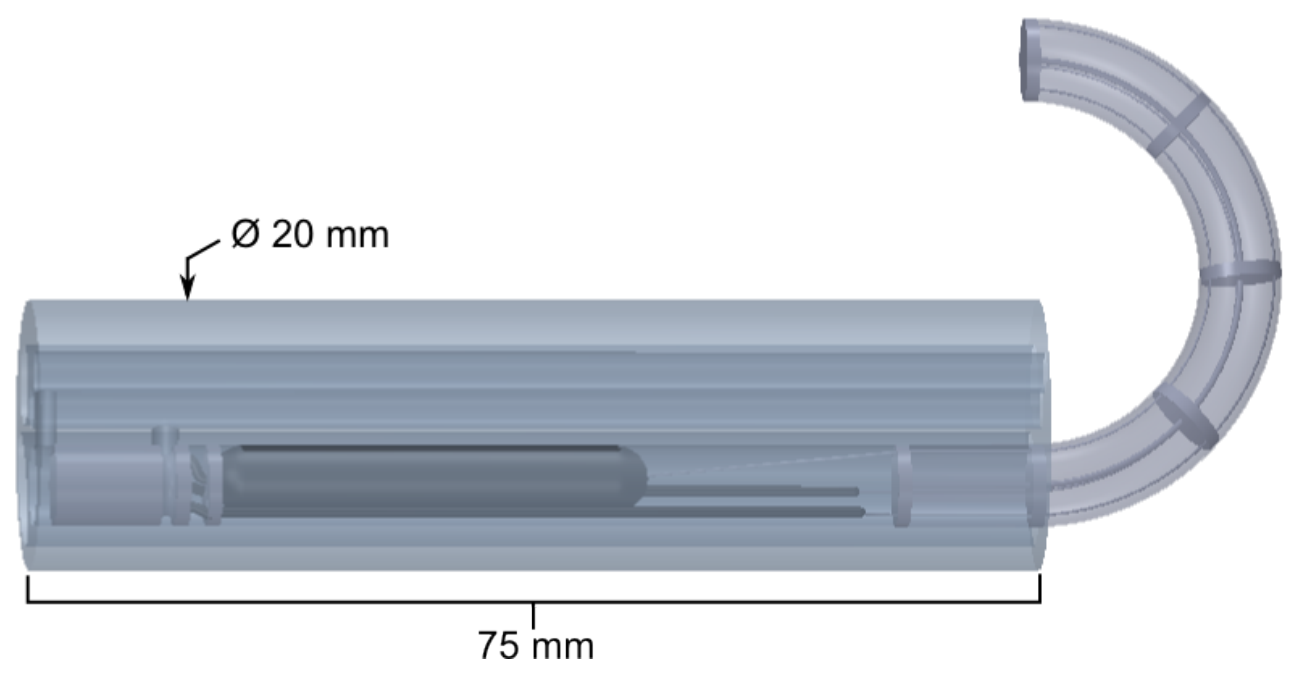

(c)

Figure 6.32: Computer rendering showing internal system components for a single manipulator section in (a) an exploded view, (b) assembled in place, and (c) assembled within the working channel of an endoscope. 


\section{Chapter 7}

\section{Conclusions and Future Work}

\subsection{Review}

The hypothesis of the research presented here was that hydraulic power can be scaled and adapted for use in embedded surgical robots for minimally invasive surgery. To test this hypothesis, it was first necessary to identify the requirements for novel minimally invasive surgical robots and the needs that exist in the field through experimentation and a review of existing and developing devices. A vision for the proposed solution was then laid out and the major considerations specified. Under this vision, it was necessary to consider the specifics of each of the relevant hydraulic components that must be present to assemble a surgical robot. Finally, through analysis and testing of these components several things were learned about the realization of a hydraulic surgical robot and provided much insight to further developments in this field.

While the future of NOTES is in question by many, the progression of gastrointestinal surgical intervention methodologies over the years traces a clear path based around the continuous reduction in incision number and size. Following 
this progression through current developments makes a compelling argument that single-incision surgery and eventually natural orifice surgery are inevitable if the surgical practice is to continue to progress. However, for this to be possible, the tools that enable these procedures must be available and fully qualified. The transgastric approach for NOTES is often considered the most challenging from a technological viewpoint and thus is the subject of much research and development.

The first chapter began by describing the history of surgical intervention and the development of minimally invasive surgical techniques. The progression of the surgical practice in relation to the abdominal cavity from traditional open access techniques to emerging natural orifice procedures was presented. The tools that will enable these novel procedures are developing rapidly. A review of these technologies was conducted and the strengths and weaknesses described as a means of developing the design path for this research. The rest of the chapter summarized the motivation for this research, stated the problem to be overcome, and laid out a vision for the design of a hydraulic surgical robot for minimally invasive surgical interventions.

The next chapter described the design requirements for the surgical robot designed for use in natural orifice transgastric endoscopic surgeries. First, the requirements for load capacity at the tool tip were investigated. As it was found that little information existed in the literature to describe this requirement, a set of experiments was conducted to quantify this value. The results of these experiments were presented. Following this, a review of the literature was used to evaluate design requirements for the surgical workspace as well as considerations for safety and biocompatibility for the design. These considerations helped to guide decisions made during the design process.

Chapter 3 provided a detailed description and analysis of the selected backbone structure. A model using the principle of minimum potential energy and inverse kinematics was presented to predict the behavior of the backbone. The model was 
then experimentally validated to demonstrate that it is capable of producing accurate beam shape predictions under most circumstances. Recommendations were provided as to how to best utilize the model under the prescribed assumptions.

In Chapter 4, the selection of an actuator to drive the manipulator was presented. The performance of the actuator was predicted analytically and confirmed experimentally. Further, an optimization algorithm was used to determine the ideal parameters necessary for fabrication of an actuator which maximizes range of motion within specified constraints placed on actuator diameter and force capability. This was accomplished through modeling of the stresses present within the actuator using finite element analysis. It was then demonstrated that the requirements placed upon the actuator can be met using the given design.

Chapter 5 described the method of providing control input to the system by way of a control valve. To meet the needs of the presented device within the constraints of the application, a novel valve design was conceived. Design parameters that are necessary to achieve desired valve performance were calculated. Two realizations of the design were fabricated at a larger scale to provide a means of evaluating the design performance and confirming the hydraulic principles used to develop the design. The results presented suggest that the design can be scaled to a size appropriate for application to the field of minimally invasive surgery.

In Chapter 6, a description of the assembly of the novel manipulator was provided. A model is shown which combines each of the manipulator components to allow for prediction of manipulator performance. Design specifications were given for each of the components that make up the assembly such that the design is theoretically capable of meeting the requirements set forth in Chapter 2, Additionally, results from testing of a manually operated manipulator assembly were presented to demonstrate the efficacy of the design. It was shown that it was possible to achieve the desired performance objectives using this design. 


\subsection{Conclusions}

The field of surgical robotics is rapidly moving and the development of new surgical robots is taking place continuously from both academia and industry. These developments are motivated by both the expansion of laparoscopy as a method of surgical intervention and the exploration of new surgical methods. The only robot performing abdominal procedures on a wide scale is the da Vinci Surgical System by Intuitive Surgical. While the da Vinci robot has helped gain acceptance for robot surgery, it has several limitations such as cost, size, and mobility which have made it inaccessible to smaller, rural hospitals and for use in remote or battlefield situations. Further, the da Vinci system is not currently well adapted for newer natural orifice access techniques. However, recent developments have suggested that the company is working towards expanding the platform for these types of surgical developments [165. Beyond the da Vinci, a wide variety of novel surgical robots have been described in the literature. A number of these were presented in Section 1.1.5. While each of these devices approach the problem from a different direction, they all face the same challenges in balancing between scale and robotic capability. It is from this balance that the hypothesis of adapting hydraulic power for use in embedded surgical robotics was born.

After a review of the literature, it was found that the load requirements for performing procedures in the abdominal cavity are not well documented and much of the information available was in the form of estimation and speculation. Therefore, a series of experiments was conducted to help quantify these requirements. The common surgical tasks that were identified as likely having the highest tool force requirements included organ or tissue manipulation, suture knot tightening, and suture breaking. The results of this investigation contribute to the relatively small pool of available quantifications of tool force measurements for abdominal surgical tasks that can be found in the literature. This helps to lend more rigor to an area that has largely been taken for granted when designing novel surgical 
robots. Of particular note, when reviewing the literature available on robots in development, it is evident that many of these robots do not achieve this load capacity specification. This may limit the practical applicability of these novel robots to lighter duty activities. The results for tool force load combined with considerations for workspace, safety, and biocompatibility assembled from available resources helps to define the requirements for surgical robots and guide the design process.

A continuous backbone was selected as the structural member for the manipulator. This selection was made because a continuous backbone provides enhanced usability in complex environments or for fine motor manipulation. However, a downside to the use of a continuous beam manipulator is that its behavior under load cannot be easily predicted due to the complexities of spatial large deflections. While a variety of options for the analytical prediction of beam behavior have been demonstrated in the literature, the majority of them require a prohibitive level of computational effort to arrive at a solution. As an alternative to available solution methods, an inverse kinematics model based on the assumption that the beam would assume the configuration with the lowest potential energy was developed. The two-dimensional version of this model was validated experimentally and found to be reasonably accurate for most conditions. This model makes it possible to predict the beam shape and end forces/moments for a given set of end position and orientation parameters thus making it possible to use these predictions for further design.

The continuous backbone is driven by hydraulic actuators connected to thin wires which are routed through evenly spaced disks along the backbone. The disk at which these wires terminate determines the location where the majority of the actuator load is applied to the backbone. For this application, artificial muscle actuators were selected rather than traditional hydraulic actuators due to their ability to achieve greater force output for the same pressure and size at the expense of contraction range and a force output which varies with actuator length. This 
realization is important for a severely size constrained application. Further, the response of the muscle actuators is similar to that of human muscle tissue in terms of compliance, aiding to the safety of the manipulator during an operation. Artificial muscle actuators are typically used in pneumatic applications; however, the use of water or saline results in similar actuator performance and less risk in the case of a burst. It was not possible to prototype a muscle actuator at the design scale, and thus larger actuator prototypes were produced and confirmed the predicted actuator performance. For use in the surgical manipulator, the artificial muscle actuator works well since the limitations in diameter can be met. Further, the surgical manipulator has less stringent limitations for the length of the actuator and this helped to make the muscle actuator a feasible solution.

Flow within the system to each of the actuators is manipulated by a novel flow control valve. To arrive at a control solution for the surgical manipulator, it was necessary to find a control valve or combination of control valves that could regulate the pressure supplied to each of the three actuators that articulate a given manipulator segment. For this to be feasible, the valve must fit within the available diameter. Hydraulic valves of this size were not found to be available commercially. Therefore, the design of a novel control valve was presented. The requirements for the valve were that it be capable of proportionally regulating the pressure supplied to each of the actuators while meeting the constraints of size. Ideally the valve would provide for both differential operation of the actuators and stiffness control of the three actuators together. Several designs for this actuator were considered. The two designs presented in Section 5 make use of three dimensional motion of a manipulated part (plug or ring) that acts as a "flapper". Planar motion of the flapper opens and closes the flapper nozzle for each of the actuators differentially. The flapper can be positioned such that pressure in any one actuator is increased individually or in pairs of two. This method of control is appropriate as the actuators would typically be controlled in this way for manipulation of the robot. Axial (out-of-plane) motion of the flapper can be used for stiffness control of the 
three actuators together. A scaled prototype of each of these valve designs was produced. Testing of these prototypes revealed that the actuator pressure could be manipulated as expected through manual operation of the valve. However, for the plug based design, the available range of this pressure control was smaller than expected most likely due to imperfections in the rapid prototyping based fabrication method. While it was not possible to test the use of the driving coils in valve operation, testing of the coils independently found that the small diameter coils were capable of manipulating the plug without overheating when a steel core was used. Further, when submerged in water, the current capacity of the coil was increased significantly. Thus, in practice the coil should provide sufficient input for valve manipulation. The ring based design proved much more capable at increasing the pressure at the actuator feed points. This is likely due in part to the larger manipulated component which is more easily handled and the fact that the valve could be fabricated out of aluminum through traditional machining techniques. With the ring valve connected to the artificial muscle actuator it was found that, on average, the actuator could be inflated in 0.35 seconds when the flapper nozzle is fully blocked by the ring. This suggests that the orifice size for the desired flow rate is appropriate and thus this valve design shows feasibility of the valve concept.

The design of a hydraulic surgical robot has proved to be a difficult endeavor with many challenges. The application of endoscopic robots to transgastric natural orifice surgery introduces severe constraints in terms of the size of the device, load capacity, and safety and biocompatibility considerations. While the design of many devices have been proposed in the literature, the majority of them do not meet several of these requirements and do not represent a viable solution for this type of surgical procedure. In theory, the application of hydraulic power to this field presents several desirable characteristics such as improved power density, greater stiffness, and faster response than comparable electromechanical or 
pneumatic designs. To map these principles to the field of surgical robotics required significant effort as hydraulic components of an appropriate size are not available commercially. Therefore it was necessary to consider alternative solutions and novel designs for the various robotic components to assemble a design that was capable of meeting the design specifications while taking advantage of hydraulic power as a system input. Through these efforts, much new information was produced and developments resulted from the research presented here. The considerations of hydraulic power for surgical robots were evaluated and are applicable to other surgical tasks where hydraulic power may be used advantageously. A quantification of the load requirements for surgical robots performing abdominal procedures was produced which will provide a guide for other researchers developing surgical robots. These values have typically been reported anecdotally in the literature and thus are a valuable resource for the field. An alternative model for predicting the behavior of continuum beams under load was developed to provide a inverse formulation for computing beam shape and end loads. This is useful as continuum beams are widely used for minimally invasive surgical manipulators as well as in a wide variety of other applications. Finally, a novel valve concept and two possible designs realizing this concept were developed. These valve designs facilitate control over the three actuators in an antagonistic arrangement. Further, the valve designs enable proportional control of the three actuators at a size scale not commercially available. In summary, the design of a novel hydraulic surgical manipulator as a summation of its parts has been performed and it has been demonstrated that fluid power can be used advantageously for the design of embedded surgical robotic tools for minimally invasive surgery. The pursuit of this research has provided many unique challenges and the work presented here has addressed many of them, as well as laid the foundation for future developments in the application of hydraulic power to the growing field of surgical robotics for minimally invasive surgery. 


\section{3 $\quad$ Future Work}

This dissertation has presented the design of a novel hydraulic surgical manipulator as a contribution to the rapidly growing body of knowledge surrounding the field of minimally invasive surgery and the tools necessary to perform such procedures. The following sections outline several opportunities for which future work could proceed, building upon the foundation of work presented here.

\subsubsection{Prototype Iteration}

As previously discussed, difficulties with the fabrication of the valve prototype prevented testing of the manipulator in a controlled manner. The next steps would then be to refine the valve prototype fabrication to achieve a working version. For the plug-based version of the valve, the primary limitations of the prototype were that the complicated geometry of the valve body required the use of a rapid prototyping process. Therefore, a fabrication of the valve at design scale should be explored. To do this it will likely be necessary to look at ways to simplify the geometry for manufacturability. Further, the use of alternative manufacturing processes may help to realize the design. In particular, the tetrahedral seat for the plug is the most difficult feature to produce. The use of electrical discharge machining may make the tetrahedral shape possible. With these considerations it is believed that producing a working prototype of the plug-based valve design will be possible.

The ring-based valve prototype demonstrated that the valve design was feasible and worked as expected when manually manipulated. The future goal for this valve prototype would be to achieve greater miniaturization through precision manufacturing. The primary benefit of miniaturization, aside from meeting the size requirements, would be to make the ring small enough in mass that it can be 
manipulated with either an electromagnetic or a piezoelectric actuator. As the scale of this valve design decreases, the tolerances of the machining operations become increasingly tight. Therefore, the use of precision micro-machining will likely be necessary to make the design scale prototype possible.

Once the valve design prototype has been produced, it will be possible to integrate it with the other components of the manipulator. Fabrication of a custom internal bladder will permit miniaturization of the artificial muscle actuator. This can be accomplished by contracting with a custom extrusion company to produce tubing at an appropriate diameter as no solutions were found to be available off-the-shelf. This will permit further testing of the system prototype to ensure that the design requirements can be met at design scale. Further, this will make it possible to test system response and find an appropriate mapping from user input to desired manipulator action.

\subsubsection{Sensing and Control}

The use of active sensing at the distal end of the surgical manipulator is one area that garners much attention within the field of surgical robotics. However, there is not yet a consensus regarding the method in which it is employed or even the necessity of its use. When it comes to force-feedback to provide a sense of touch for the surgeon who is manipulating the robot, the answer is unclear. Surgeons often proclaim that the presence of force-feedback is a necessity for robotic minimally invasive surgery, yet surgeons regularly perform procedures without this information whether it be with hand-held tools or with commercially available robots such as the da Vinci [89]. Some work has explored the use of visual or auditory feedback to relay force information or even to automatically avoid objects all together [98, 166, 167]. However, the effectiveness of these methods seem to have significant variation among individual surgeons [168]. More investigation into the methods by which this information is relayed to the surgeon and the costs 
or benefits of proving it is necessary.

Another use for sensing on the distal end of the robot is to provide position feedback to the controller. This form of feedback can be provided through active measurement of the tool joints (not possible with a continuum based robot) or possibly through other means such as strain gauge based measurements on the manipulator backbone. It may also be possible to gather this information through measurement of the actuator contraction or pressure. Further, some work has explored the use of algorithmic based optical tracking of the tool using either MRI or some other imaging modality [169]. Current commercially available robots make use of the surgeon as a means for correcting absolute positional error produced by the robot, which has been measured to be as large as $25 \mathrm{~mm}$ [79]. In this way the surgeon is able to compensate for any realized error due to tool behavior or unexpected loads and increase or decrease the magnitude of his or her input as appropriate.

It is also necessary to consider the method by which the control valve will be operated. To achieve this, a control algorithm must be developed. This development can likely build upon similar work done in areas such as human power amplifiers [170, 171], which are an appropriate solution for many applications due to their high power density and ability to accept direct human manipulation. Additionally, considering the principles of teleoperation, it is then possible to produce an output that can be scaled in both force and displacement. Tools such as these enable a virtual physical connection between the human operator and the task being performed. Additionally, through the use of force-feedback it is possible to provide an intuitive, haptic interface for the operator where they can receive information about the loads being applied to the tool and adjust the tool operation appropriately.

Similar techniques have been applied to translate human manipulation to a smaller scale system using a human power attenuator. An attenuator allows the human 
operator to manipulate the controls on a scale that is more natural while the tool performs the same motion at a more precise scale. Much work has been performed in this area along with its application to surgical robotic systems for minimally invasive surgery [72, 172, 173].

The advent of minimally invasive surgery has thrust this field into the forefront. As both surgeons and patients seek out new technologies that use smaller incisions and minimize the risk of injury, the necessity for increasingly small surgical instruments is growing. With decreasing size of surgical instrumentation, it becomes more difficult for the surgeons to manipulate these tools in a precise, controlled manner. Therefore, surgical robotic systems provide a means to achieve precise manipulation and force application while maintaining the surgeon's control over the surgical tools.

The control objective for such a system is to accept the power input by the operator at the control joystick and produce an attenuated power output at the surgical tool. Difficulties may arise in the synchronization of the input joystick and output hydraulic actuator due to model uncertainties inherent to hydraulic systems [174]. Uncertainties may be presented in the form of external disturbances, fluid leakage, and friction.

\subsubsection{User Interface}

A proper user interface is critical for the success of a surgical manipulator. As previously mentioned, the da Vinci surgical system makes use of a surgeon console that has gained widespread acceptance for the operation of a surgical robot. This type of console works well for remote operation of a surgical robot as it provides visual feedback to the surgeon, places the surgeon in an ergonomic position, and allows the surgeon to provide input to the robot. A similar surgeon console would be necessary for the manipulator described in this work. Since each segment of the 
manipulator contains two degrees of freedom, it is possible to measure user input with two sensors. These sensors could be of the form of rotary potentiometers. Using two potentiometers oriented perpendicular to each other would permit the capture of both degrees of freedom at a single location similar to a joystick. This would also permit expansion of the user input to additional segments by adding addition pairs of potentiometers. By positioning the potentiometer pairs at natural locations such as the elbow and wrist, operation of the surgical robot would be more intuitive. This method of user input would be consistent with the use of artificial muscle actuators in that the manipulator would move and behave in a familiar manner. This can lead to reduced training time for surgical students as well as reduced warm-up time before each procedure. An idealized user interface for control of a surgical robot would provide three-dimensional visual capability through a head mounted system, allow the surgeon to don and doff the console at will, and include all the necessary forms of information delivery to ensure the best possible surgical outcomes [85]. 


\section{References}

[1] J.M. Levine. Historical notes on pressure ulcers: The cure of Ambrose Paré. Advances in Skin \&6 Wound Care, 5(2):23, 1992.

[2] H. Ellis. A history of surgery. Cambridge University Press, 2001.

[3] K.H. Fuchs. Minimally invasive surgery. Endoscopy, 34(2):154-159, 2002.

[4] T.N. Robinson and G.V. Stiegmann. Minimally invasive surgery. Endoscopy, 36(1):48-51, 2004.

[5] M. Hatzinger, S.T. Kwon, S. Langbein, S. Kamp, A. Häcker, and P. Alken. Hans Christian Jacobaeus: Inventor of human laparoscopy and thoracoscopy. Journal of Endourology, 20(11):848-850, 2006.

[6] A. Lehman, M. Rentschler, S. Farritor, and D. Oleynikov. The current state of miniature in vivo laparoscopic robotics. Journal of Robotic Surgery, 1(1):45-49, 2007.

[7] F. Tendick, S.S. Sastry, R.S. Fearing, and M. Cohn. Applications of micromechatronics in minimally invasive surgery. IEEE/ASME Transactions on Mechatronics, 3(1):34-42, 1998.

[8] T. Falcone and J.M. Goldberg. Robotic surgery. Clinical Obstetrics and Gynecology, 46(1):37-43, 2003.

[9] R.A. Li, J. Jensen, and J.C. Bowersox. Microvascular anastomoses performed in rats using a microsurgical telemanipulator. Computer Aided Surgery, 5(5):326-332, 2000.

[10] M.E. Rentschler, A. Hadzialic, J. Dumpert, S.R. Platt, S. Farritor, and D. Oleynikov. In vivo robots for laparoscopic surgery. Studies in Health Technology and Informatics, 98:316-322, 2004. 
[11] The laparascopic gallbladder surgery procedure. http://www . laparoscopicsurgeryinfo.com./procedure.htm. Accessed: December 2010 .

[12] E.J. Reddick, D.O. Olsen, J.F. Daniell, W.B. Saye, B. McKernan, W. Miller, and M. Hoback. Laparoscopic laser cholecystectomy. Laser Medicine and Surgery News and Advances, 7(1):38-40, 1989.

[13] NIH Consensus Development Panel on Gallstones and Laparoscopic Cholecystectomy. Gallstones and Laparoscopic Cholecystectomy. Journal of the American Medical Association, 269(8):1018-1024, 1993.

[14] J.H. Kaouk, G.P. Haber, R.K. Goel, M.M. Desai, M. Aron, R.R. Rackley, C. Moore, and I.S. Gill. Single-port laparoscopic surgery in urology: Initial experience. Urology, 71(1):3-6, 2008.

[15] K. Ahmed, T. Wang, V. Patel, K. Nagpal, J. Clark, M. Ali, S. Deeba, H. Ashrafian, A. Darzi, T. Athanasiou, and P. Paraskeva. The role of singleincision laparoscopic surgery in abdominal and pelvic surgery: A systematic review. Surgical Endoscopy, pages 1-19, 2010.

[16] Single port access. http://www.opnotes.com/. Accessed: November 2010.

[17] J.D. Paulson, J.W. Ross, and S. El-Sahwi. Development of flexible culdoscopy. The Journal of the American Association of Gynecologic Laparoscopists, 6(4):487-490, 1999.

[18] D. Rattner and A. Kalloo. ASGE/SAGES working group on natural orifice translumenal endoscopic surgery. Surgical Endoscopy, 20(2):329-333, 2006.

[19] A.N. Kalloo, V.K. Singh, S.B. Jagannath, H. Niiyama, S.L. Hill, C.A. Vaughn, C.A. Magee, and S.V. Kantsevoy. Flexible transgastric peritoneoscopy: A novel approach to diagnostic and therapeutic interventions in the peritoneal cavity. Gastrointestinal Endoscopy, 60(1):114-117, 2004.

[20] D.A. Rini. Trans-gastric ligation of fallopian tubes. http://www. hopkinsmedicine.org/medart/drini2.htm. Accessed: November 2010.

[21] T.H. Baron. Natural orifice transluminal endoscopic surgery. The British Journal of Surgery, 94(1):1-2, 2007.

[22] L. Barclay. Natural orifice translumenal endoscopic surgery may allow incisionless operations. Medscape Medical News, 2008. http://www medscape. com/viewarticle/578108.

[23] G.H. Ballantyne. Robotic surgery, telerobotic surgery, telepresence, and telementoring. Surgical Endoscopy, 16(10):1389-1402, 2002. 
[24] G. Hubens, H. Coveliers, L. Balliu, M. Ruppert, and W. Vaneerdeweg. A performance study comparing manual and robotically assisted laparoscopic surgery using the da Vinci system. Surgical Endoscopy, 17(10):1595-1599, 2003.

[25] H.M. Shao, J.Y. Chen, T.K. Truong, I.S. Reed, and Y.S. Kwoh. A new CTaided robotic stereotaxis system. In Proceedings of the Annual Symposium on Computer Application in Medical Care, page 668. American Medical Informatics Association, 1985.

[26] Y.S. Kwoh, J. Hou, and E. Jonckheere. A robot with improved absolute positioning accuracy for CT guided stereotactic brain surgery. IEEE Transactions on Biomedical Engineering, 35(2):153-160, 1988.

[27] E.H. Spencer. The ROBODOC clinical trial: A robotic assistant for total hip arthroplasty. Orthopaedic Nursing, 15(1):9-14, 1996.

[28] A.R. Lanfranco, A.E. Castellanos, J.P. Desai, and W.C. Meyers. Robotic surgery: A current perspective. Annals of Surgery, 239(1):14-21, 2004.

[29] da Vinci Si system. http://www.intuitivesurgical.com/company/ media/images/davinci_si_images.html. Accessed: November 2010.

[30] Intuitive Surgical Inc. Company backgrounder. http://www . intuitivesurgical.com/company/media/media_kit/871072-K_ Company_Backgrounder.pdf. Accessed: December 2012.

[31] R. Peplinski and R. Rhodes. Economic aspects of starting a Da Vinci robotic surgery program. In Robotic Urology, pages 253-261. Springer Berlin Heidelberg, 2008.

[32] R. Rothstein, J. Rosen, and J.S. Young. Improving efficiency in endoscopy with robotic technology. Gastrointestinal Endoscopy Clinics of North America, 14(4):679-696, 2004.

[33] B.C. Shah, S.L. Buettner, A.C. Lehman, S.M. Farritor, and D. Oleynikov. Miniature in vivo robotics and novel robotic surgical platforms. Urologic Clinics of North America, 36(2):251-263, 2009.

[34] A. Slatkin, J. Burdick, and W. Grundfest. The development of a robotic endoscope. In Experimental Robotics IV, volume 223, pages 161-169. Springer Berlin Heidelberg, 1997.

[35] U. Scarfogliero, M. Piccigallo, C. Quaglia, G. Petroni, P. Valdastri, A. Menciassi, and P. Dario. Bimanual Robot for single-port laparoscopic surgery with on-board actuation. In G.Z. Yang and A. Darzi, editors, Proceedings 
of The Hamlyn Symposium on Medical Robotics, pages 36-37, London, UK, 2010. The Royal Society.

[36] G. Petroni, M. Niccolini, S. Caccavaro, C. Quaglia, A. Menciassi, S. Schostek, G. Basili, O. Goletti, M.O. Schurr, and P. Dario. A novel robotic system for single-port laparoscopic surgery: preliminary experience. Surgical Endoscopy, pages 1-6, 2013.

[37] M. Rentschler, J. Dumpert, S. Platt, S. Farritor, and D. Oleynikov. Natural orifice surgery with an endoluminal mobile robot. Surgical Endoscopy, 21(7):1212-1215, 2007.

[38] D.J. Scott, S. Tang, R. Fernandez, R. Bergs, M.T. Goova, I. Zeltser, F.J. Kehdy, and J.A. Cadeddu. Completely transvaginal NOTES cholecystectomy using magnetically anchored instruments. Surgical Endoscopy, 21(12):23082316, 2007.

[39] A.C. Lehman, K.A. Berg, J. Dumpert, N.A. Wood, A.Q. Visty, M.E. Rentschler, S.R. Platt, S.M. Farritor, and D. Oleynikov. Surgery with cooperative robots. Computer Aided Surgery, 13(2):95-105, 2008.

[40] A.C. Lehman, J. Dumpert, N.A. Wood, A.Q. Visty, S.M. Farritor, B. Varnell, and D. Oleynikov. Natural orifice translumenal endoscopic surgery with a miniature in vivo surgical robot. Surgical Endoscopy, 23(7):1649, 2009.

[41] A.C. Lehman, J. Dumpert, N.A. Wood, L. Redden, A.Q. Visty, S. Farritor, B. Varnell, and D. Oleynikov. Natural orifice cholecystectomy using a miniature robot. Surgical Endoscopy, 23(2):260-266, 2009.

[42] M.E. Rentschler, S.R. Platt, K.R. Berg, J. Dumpert, D.R. Oleynikov, and S.M. Farritor. Miniature in vivo robots for remote and harsh environments. IEEE Transactions on Information Technology in Biomedicine, 12(1):66-75, 2008.

[43] Y. Liu and J. Liu. Surgical robotics: A look-back of latest advancement and bio-inspired ways to tackle existing challenges. Frontiers of Mechanical Engineering, 7(4):376-384, 2012.

[44] J.L. Ponsky. Endoluminal surgery: Past, present and future. Surgical Endoscopy, 20:500-502, 2006.

[45] D.J. Abbott, C. Becke, R.I. Rothstein, and W.J. Peine. Design of an endoluminal NOTES robotic system. In Proceedings of the 2007 IEEE/RSJ International Conference on Intelligent Robots and Systems, pages 410-416, 2007. 
[46] Y. Bar-Cohen. Biomimeticsusing nature to inspire human innovation. Bioinspiration $\mathcal{E}$ Biomimetics, 1(1):1-12, 2006.

[47] L.S. Cowan and I.D. Walker. The importance of continuous and discrete elements in continuum robots. International Journal of Advanced Robotic Systems, 10(165), 2013.

[48] S.J. Phee, S.C. Low, Z.L. Sun, K.Y. Ho, W.M. Huang, and Z.M. Thant. Robotic system for no-scar gastrointestinal surgery. The International Journal of Medical Robotics and Computer Assisted Surgery, 4(1):15-22, 2008.

[49] S.J. Phee, K.Y. Ho, D. Lomanto, S.C. Low, V.A. Huynh, A.P. Kencana, K. Yang, Z.L. Sun, and S.C.S. Chung. Natural orifice transgastric endoscopic wedge hepatic resection in an experimental model using an intuitively controlled master and slave transluminal endoscopic robot (master). Surgical endoscopy, 24(9):2293-2298, 2010.

[50] Y. Kobayashi, Y. Tomono, Y. Sekiguchi, H. Watanabe, K. Toyoda, K. Konishi, M. Tomikawa, S. Ieiri, K. Tanoue, M. Hashizume, and M.G. Fujie. A surgical robot with vision field control for single port endoscopic surgery. The International Journal of Medical Robotics and Computer Assisted Surgery, 6(4):454-464, 2010.

[51] J. Shang, D.P. Noonan, C. Payne, J. Clark, M.H. Sodergren, A. Darzi, and G.-Z. Yang. An articulated universal joint based flexible access robot for minimally invasive surgery. In 2011 IEEE International Conference on Robotics and Automation, pages 1147-1152. IEEE, 2011.

[52] S.J. Bardaro and L. Swanström. Development of advanced endoscopes for natural orifice transluminal endoscopic surgery (NOTES). Minimally Invasive Therapy and Allied Technologies, 15(6):378-383, 2006.

[53] G.O. Spaun, B. Zheng, and L.L. Swanström. A multitasking platform for natural orifice translumenal endoscopic surgery (NOTES): A benchtop comparison of a new device for flexible endoscopic surgery and a standard dualchannel endoscope. Surgical Endoscopy, 23(12):2720-2727, 2009.

[54] K.-W. Kwok, K.H. Tsoi, V. Vitiello, J. Clark, G.C.T. Chow, W. Luk, and G.-Z. Yang. Dimensionality reduction in controlling articulated snake robot for endoscopy under dynamic active constraints. IEEE Transactions on Robotics, 29:15-31, 2013.

[55] R.J. Webster, A.M. Okamura, and N.J. Cowan. Toward active cannulas: Miniature snake-like surgical robots. In Proceedings of the 2006 IEEE/RSJ International Conference on Intelligent Robots and Systems, pages 28572863, 2006. 
[56] D.B. Comber, D. Cardona, R.J. Webster III, and E.J. Barth. Sliding mode control of an mri-compatible pneumatically actuated robot. In Proceedings of the Fluid Power and Motion Control Symposium, pages 283-293, 2012.

[57] D.C. Cardona. A MRI compatible concentric tube continuum robot with pneumatic actuation. Master's thesis, Vanderbilt University, 2012.

[58] N. Simaan, K. Xu, A. Kapoor, W. Wei, P. Kazanzides, P. Flint, and R. Taylor. Design and integration of a telerobotic system for minimally invasive surgery of the throat. The International Journal of Robotics Research, 28(9):1134-1153, 2009.

[59] G. Dogangil, B.L. Davies, and F. Rodriguez y Baena. A review of medical robotics for minimally invasive soft tissue surgery. Proceedings of the Institution of Mechanical Engineers, Part H: Journal of Engineering in Medicine, 224(5):653-679, 2010.

[60] J. Ding, K. Xu, R. Goldman, P. Allen, D. Fowler, and N. Simaan. Design, simulation and evaluation of kinematic alternatives for insertable robotic effectors platforms in single port access surgery. In 2010 IEEE International Conference on Robotics and Automation, pages 1053-1058. IEEE, 2010.

[61] Kai Xu, Jiangran Zhao, and AlbertJ. Shih. Development of an endoscopic continuum robot to enable transgastric surgical obesity treatment. In ChunYi Su, Subhash Rakheja, and Honghai Liu, editors, Intelligent Robotics and Applications, volume 7506 of Lecture Notes in Computer Science, pages 589-600. Springer Berlin Heidelberg, 2012.

[62] G.S. Chirikjian. Hyper-redundant manipulator dynamics: A continuum approximation. Advanced Robotics, 9(3):217-243, 1995.

[63] F. Matsuno and H. Sato. Trajectory tracking control of snake robots based on dynamic model. In Proceedings of the 2005 IEEE International Conference on Robotics and Automation, pages 3029-3034. IEEE, 2005.

[64] B. He, Z. Wang, Q. Li, H. Xie, and R. Shen. An analytic method for the kinematics and dynamics of a multiple-backbone continuum robot. International Journal of Advanced Robotic Systems, 10(84), 2013.

[65] Hiromi Mochiyama and Takahiro Suzuki. Kinematics and dynamics of a cable-like hyper-flexible manipulator. In Proceedings of the 2003 IEEE International Conference on Robotics and Automation, volume 3, pages 36723677. IEEE, 2003. 
[66] E. Tatlicioglu, I.D. Walker, and D.M. Dawson. Dynamic modelling for planar extensible continuum robot manipulators. In Proceedings of the 2007 IEEE International Conference on Robotics and Automation, pages 13571362. IEEE, 2007.

[67] D.B. Camarillo, C.F. Milne, C.R. Carlson, M.R. Zinn, and J.K. Salisbury. Mechanics modeling of tendon-driven continuum manipulators. IEEE Transactions on Robotics, 24(6):1262-1273, 2008.

[68] D. Trivedi, A. Lotfi, and C.D. Rahn. Geometrically exact models for soft robotic manipulators. IEEE Transactions on Robotics, 24(4):773-780, 2008.

[69] Z. Wang, Z. Sun, and S.J. Phee. Modeling tendon-sheath mechanism with flexible configurations for robot control. Robotica, pages 1-12, 2013.

[70] J. Rosen, B. Hannaford, M.P. MacFarlane, and M.N. Sinanan. Force controlled and teleoperated endoscopic grasper for minimally invasive surgery: Experimental performance evaluation. IEEE Transactions on Biomedical Engineering, 46(10):1212-1221, 1999.

[71] E.P. Westebring-van der Putten, J.J. van den Dobbelsteen, R.H.M. Goossens, J.J. Jakimowicz, and J. Dankelman. Effect of laparoscopic grasper force transmission ratio on grasp control. Surgical Endoscopy, 23(4):818-824, 2009.

[72] S. Wang, J. Ding, J. Yun, Q. Li, and B. Han. A robotic system with force feedback for micro-surgery. In Proceedings of the 2005 IEEE International Conference on Robotics and Automation, pages 199-204, 2005.

[73] L.L. Swanström, R. Kozarek, P.J. Pasricha, S. Gross, D. Birkett, P.O. Park, V. Saadat, R. Ewers, and P. Swain. Development of a new access device for transgastric surgery. Journal of Gastrointestinal Surgery, 9(8):1129-1137, 2005 .

[74] J.J. Pippenger and T.G. Hicks. Industrial hydraulics. McGraw-Hill, 1979.

[75] G. Granosik and J. Borenstein. Pneumatic actuators for serpentine robot. In Climbing and Walking Robots, pages 719-726. Springer Berlin Heidelberg, 2006.

[76] L.J. Love, R.F. Lind, and J.F. Jansen. Mesofluidic actuation for articulated finger and hand prosthetics. In Proceedings of the 2009 IEEE/RSJ International Conference on Intelligent Robots and Systems, pages 2586-2591. IEEE Press, 2009. 
[77] C.M. Ho and Y.C. Tai. Micro-electro-mechanical-systems (MEMS) and fluid flows. Annual Review of Fluid Mechanics, 30(1):579-612, 1998.

[78] G.M. Whitesides. The origins and the future of microfluidics. Nature, 442(7101):368-373, 2006.

[79] A. Reiter, P.K. Allen, and T. Zhao. Feature classification for tracking articulated surgical tools. In Medical Image Computing and Computer-Assisted Intervention, pages 592-600. Springer, 2012.

[80] J. Peirs. Design of miniature parallel manipulators for integration in a selfpropelling endoscope. Sensors and Actuators A: Physical, 85(1-3):409-417, 2000.

[81] Y. Bailly and Y. Amirat. Modeling and control of a hybrid continuum active catheter for aortic aneurysm treatment. In Proceedings of the 2005 IEEE International Conference on Robotics and Automation, pages 924-929. IEEE, 2005.

[82] C. Raoufi, A.A. Goldenberg, and W. Kucharczyk. A new hydraulically/pneumatically actuated MR-compatible robot for MRI-guided neurosurgery. In The 2nd International Conference on Bioinformatics and Biomedical Engineering, pages 2232-2235. IEEE, 2008.

[83] J. Xia and W.K. Durfee. Analysis of small-scale hydraulic actuation systems. Journal of Mechanical Design, 2013.

[84] J. Meade. JCM Medical: Some selected projects. http://jcmmedical. com/Selected\%20Projects.html. Accessed: August 2013.

[85] A. Simorov, R.S. Otte, and D. Kopietz, C.Mand Oleynikov. Review of surgical robotics user interface: what is the best way to control robotic surgery? Surgical endoscopy, 26(8):2117-2125, 2012.

[86] I.A. Gravagne and I.D. Walker. Kinematic transformations for remotelyactuated planar continuum robots. In Proceedings of the IEEE International Conference on Robotics and Automation, volume 1, pages 19-26. IEEE, 2000 .

[87] C. Li and C.D. Rahn. Design of continuous backbone, cable-driven robots. Journal of Mechanical Design, 124:265, 2002.

[88] N. Simaan, R. Taylor, and P. Flint. A dexterous system for laryngeal surgery. In Proceedings of the 2004 IEEE International Conference on Robotics and Automation, volume 1, pages 351-357. IEEE, 2004. 
[89] C.R. Wagner, R.D. Howe, and N. Stylopoulos. The role of force feedback in surgery: analysis of blunt dissection. In Proceedings of the International Symposium on Haptic Interfaces for Virtual Environment and Teleoperator Systems, pages 73-79, Los Alamitos, CA, USA, 2002. IEEE Computer Society.

[90] J. Rosen, M. MacFarlane, C. Richards, B. Hannaford, and M. Sinanan. Surgeon-tool force/torque signatures-evaluation of surgical skills in minimally invasive surgery. In J.D. Westwood, H.M. Hoffman, R.A. Robb, and D. Stredney, editors, Proceedings of Medicine Meets Virtual Reality, MMVR7, pages 290-296, Amsterdam, Netherlands, 1999. IOS Press.

[91] A.K. Morimoto, R.D. Foral, J.L. Kuhlman, K.A. Zucker, M.J. Curet, T. Bocklage, T.I. MacFarlane, and L. Kory. Force sensor for laparoscopic Babcock. Studies in Health Technology and Informatics, 39:354, 1997.

[92] U. Seibold, B. Kubler, and G. Hirzinger. Prototype of instrument for minimally invasive surgery with 6 -axis force sensing capability. In Proceedings of the 2005 IEEE International Conference on Robotics and Automation, pages 496-501. IEEE, 2006.

[93] R.P. Brown. Knotting technique and suture materials. British Journal of Surgery, 79(5):399-400, 1992.

[94] F.J. Carter, T.G. Frank, P.J. Davies, and A. Cuschieri. Puncture forces of solid organ surfaces. Surgical endoscopy, 14(9):783-786, 2000.

[95] F.J. Carter, T.G. Frank, P.J. Davies, D. McLean, and A. Cuschieri. Measurements and modelling of the compliance of human and porcine organs. Medical Image Analysis, 5(4):231-236, 2001.

[96] P.J. Davies, F.J. Carter, and A. Cuschieri. Mathematical modelling for keyhole surgery simulations: A biomechanical model for spleen tissue. IMA Journal of Applied Mathematics, 67(1):41-67, 2002.

[97] M. Kitagawa, A. Okamura, B. Bethea, V. Gott, and W. Baumgartner. Analysis of suture manipulation forces for teleoperation with force feedback. In T. Dohi and R. Kikinis, editors, Proceedings of Medical Image Computing and Computer-Assisted Intervention, pages 155-162. Springer-Verlag Berlin Heidelberg, 2002.

[98] M. Kitagawa, D. Dokko, A.M. Okamura, and D.D. Yuh. Effect of sensory substitution on suture-manipulation forces for robotic surgical systems. Journal of Thoracic and Cardiovascular Surgery, 129(1):151-158, 2005. 
[99] A.M. Okamura, C. Simone, and M.D. O'Leary. Force modeling for needle insertion into soft tissue. IEEE Transactions on Biomedical Engineering, 51(10):1707-1716, 2004.

[100] A. Tamura, K. Omori, K. Miki, J.B. Lee, K.H. Yang, and A.I. King. Mechanical characterization of porcine abdominal organs. Stapp car crash journal, 46:55-69, 2002.

[101] J. Kyle, J.A.R. Smith, and D.H. Johnston, editors. Pye's surgical handicraft. Butterworth-Heinemann, Oxford, 22nd edition, 1992.

[102] University of Minnesota. The visible heart laboratory. http://www . vhlab. umn.edu/. Accessed: May 2011.

[103] S. De, J. Rosen, A. Dagan, P. Swanson, M. Sinanan, and B. Hannaford. Tissue damage due to mechanical stresses as applied during minimally invasive surgery. International Journal of Robotics Research, 26:1159-1171, 2007.

[104] I.A. Trail, E.S. Powell, and J. Noble. An evaluation of suture materials used in tendon surgery. The Journal of Hand Surgery, 14(4):422-427, 1989.

[105] T.M. Kowalewski. Real-time Quantitiative Assessment of Surgical Skill. $\mathrm{PhD}$ thesis, University of Washington, 2012.

[106] M.J.H. Lum, J. Rosen, M.N. Sinanan, and B. Hannaford. Optimization of a spherical mechanism for a minimally invasive surgical robot: Theoretical and experimental approaches. IEEE Transactions on Biomedical Engineering, 53(7):1440-1445, 2006.

[107] V.K. Narula, J.W. Hazey, D.B. Renton, K.M. Reavis, C.M. Paul, K.E. Hinshaw, B.J. Needleman, D.J. Mikami, E.C. Ellison, and W.S. Melvin. Transgastric instrumentation and bacterial contamination of the peritoneal cavity. Surgical Endoscopy, 22(3):605-611, 2008.

[108] D.N. Papachristou and R. Barters. Resection of the liver with a water jet. British Journal of Surgery, 69(2):93-94, 1982.

[109] J. Rosen, J.D. Brown, S. De, M. Sinanan, and B. Hannaford. Biomechanical properties of abdominal organs in vivo and postmortem under compression loads. Journal of Biomechanical Engineering, 130(2), 2008.

[110] S. De, J. Rosen, A. Dagan, B. Hannaford, P. Swanson, and M. Sinanan. Assessment of tissue damage due to mechanical stresses. The International Journal of Robotics Research, 26(11-12):1159-1171, 2007. 
[111] S.P. Rodrigues, T. Horeman, J. Dankelman, J.J. van den Dobbelsteen, and F.W. Jansen. Suturing intraabdominal organs: When do we cause tissue damage? Surgical Endoscopy, pages 1-5, 2012.

[112] I.D. Walker. Robot strings: Long, thin continuum robots. In 2013 IEEE Aerospace Conference, pages 1-12. IEEE, 2013.

[113] R.J. Webster III and B.A. Jones. Design and kinematic modeling of constant curvature continuum robots: A review. The International Journal of Robotics Research, 29(13):1661-1683, 2010.

[114] V.C. Anderson and R.C. Horn. Tensor arm manipulator design. ASME Transactions, 67-DE-57:1-12, 1967.

[115] D.C. Rucker and R.J. Webster. Statics and dynamics of continuum robots with general tendon routing and external loading. IEEE Transactions on Robotics, 27(6):1033-1044, 2011.

[116] S.S. Antman. Nonlinear Problems of Elasticity. Springer, 2nd edition, 2005.

[117] C. Felippa. Advanced variational methods in mechanics: The bernoullieuler beam. http://wWw.colorado.edu/engineering/CAS/courses.d/ AVMM.d/AVMM.Ch08.d/AVMM.Ch08.pdf. Accessed: January 2013.

[118] R.C. Hibbeler. Mechanics of Materials. Pearson Prentice Hall, Upper Saddle River, NJ, 6th edition, 2005.

[119] I.A. Gravagne, C.D. Rahn, and I.D. Walker. Large deflection dynamics and control for planar continuum robots. IEEE/ASME Transactions on Mechatronics, 8(2):299-307, 2003.

[120] A. Bedford. Hamilton's Principle in Continuum Mechanics. Pitman Advanced Publishing Program, 1985.

[121] G.S. Chirikjian and J.W. Burdick. A modal approach to hyper-redundant manipulator kinematics. IEEE Transactions on Robotics and Automation, 10(3):343-354, 1994.

[122] G.S. Chirikjian and J.W. Burdick. The kinematics of hyper-redundant robot locomotion. IEEE Transactions on Robotics and Automation, 11(6):781793, 1995.

[123] MatWeb. Nitinol - NiTi shape memory alloy; low-temperature phase. http://matweb.com/search/datasheet.aspx?matguid= 44afc7d3c6eb4829bc2df27884fd2d6c\&n=1. Accessed: November 2012. 
[124] R.G.D. Steel and J.H. Torrie. Principles and Procedures of Statistics with Special Reference to the Biological Sciences. McGraw-Hill, 1960.

[125] H.F. Schulte. The characteristics of the McKibben artificial muscle. In The Application of External Power in Prosthetics and Orthotics, pages 94-115. National Academy of Sciences National Research Council, 1961.

[126] G.K. Klute and B. Hannaford. Accounting for elastic energy storage in mckibben artificial muscle actuators. Journal of Dynamic Systems, Measurement, and Control, 122(2):386-388, 2000.

[127] N. Tsagarakis and D.G. Caldwell. Improved modelling and assessment of pneumatic muscle actuators. In Proceedings of the 2000 IEEE International Conference on Robotics and Automation, volume 4, pages 3641-3646, 2000.

[128] S. Davis, N. Tsagarakis, J. Canderle, and D.G. Caldwell. Enhanced modelling and performance in braided pneumatic muscle actuators. The International Journal of Robotics Research, 22(3-4):213-227, 2003.

[129] C.-P. Chou and B. Hannaford. Measurement and modeling of McKibben pneumatic artificial muscles. IEEE Transactions on Robotics and Automation, 12(1):90-102, 1996.

[130] B. Tondu, V. Boitier, and P. Lopez. Théorie d'un muscle artificiel pneumatique et application à la modélisation du muscle artificiel de McKibben. Comptes rendus de l'Académie des Sciences, 320:105-114, 1996.

[131] S. Davis and D.G. Caldwell. Braid effects on contractile range and friction modeling in pneumatic muscle actuators. The International Journal of Robotics Research, 25(4):359-369, 2006.

[132] E.W.S. Hagan, M.N. Charalambides, C.T. Young, T.J.S. Learner, and S. Hackney. Tensile properties of latex paint films with tio2 pigment. $\mathrm{Me}$ chanics of Time-Dependent Materials, 13(2):149-161, 2009.

[133] D.R. Berg. Abaqus script for diamond shaped pressurized plate. http://github.com/devinberg/ Abaqus-script-for-diamond-shaped-pressurized-plate, 2013. Accessed: June 2013.

[134] D.R. Berg. FEA results for a diamond shaped pressurized plate of variable dimensions. http://dx.doi.org/10.6084/m9.figshare.710602, 2013. Accessed: May 2013.

[135] S. Omeroglu. The effect of braiding parameters on the mechanical properties of braided ropes. Fibres and Textiles in Eastern Europe, 14(4):53, 2006. 
[136] D. Trivedi, C.D. Rahn, W.M. Kier, and I.D. Walker. Soft robotics: Biological inspiration, state of the art, and future research. Applied Bionics and Biomechanics, 5(3):99-117, 2008.

[137] M. Linjama and M. Vilenius. Digital hydraulics - Towards perfect valve technology. In Proceedings of the Tenth Scandinavian International Conference on Fluid Power, volume 1, pages 181-196, 2007.

[138] V. Studer, G. Hang, A. Pandolfi, M. Ortiz, W.F. Anderson, and S.R. Quake. Scaling properties of a low-actuation pressure microfluidic valve. Journal of Applied Physics, 95:393-398, 2004.

[139] A. Luque, J.M. Quero, C. Hibert, P. Flückiger, and A.M. Gañán-Calvo. Integrable silicon microfluidic valve with pneumatic actuation. Sensors and Actuators A: Physical, 118(1):144-151, 2005.

[140] J.Y. Baek, J.Y. Park, J.I. Ju, T.S. Lee, and S.H. Lee. A pneumatically controllable flexible and polymeric microfluidic valve fabricated via in situ development. Journal of Micromechanics and Microengineering, 15:10151020, 2005.

[141] P. Selvaganapathy, E.T. Carlen, and C.H. Mastrangelo. Electrothermally actuated inline microfluidic valve. Sensors and Actuators A: Physical, 104(3):275-282, 2003.

[142] M.A. Huff, M.S. Mettner, T.A. Lober, and M.A. Schmidt. A pressurebalanced electrostatically-actuated microvalve. In IEEE Solid-State Sensor and Actuator Workshop, 4th Technical Digest, pages 123-127, 1990.

[143] T.L. Sounart and T.A. Michalske. Electrostatic actuation without electrolysis in microfluidic MEMS. In 12th International Conference on Transducers, Solid-State Sensors, Actuators and Microsystems, pages 615-618, 2003.

[144] T. Ohnstein, T. Fukiura, J. Ridley, and U. Bonne. Micromachined silicon microvalve. In Proceedings of IEEE Conference on Micro Electro Mechanical Systems, pages 95-98, 1990.

[145] N. Fikru and T.R. Chase. A review of MEMS based pneumatic valves. In 2011 Proceedings of the 52nd National Conference on Fluid Power, pages 271-282, Las Vegas, NV, March 2011. IEEE Press.

[146] C. Fu, Z. Rummler, and W. Schomburg. Magnetically driven micro ball valves fabricated by multilayer adhesive film bonding. Journal of Micromechanics and Microengineering, 13(4):S96, 2003. 
[147] P. Pernod, V. Preobrazhensky, A. Merlen, O. Ducloux, A. Talbi, L. Gimeno, R. Viard, and N. Tiercelin. MEMS magneto-mechanical microvalves (MMMS) for aerodynamic active flow control. Journal of Magnetism and Magnetic Materials, 322(9):1642-1646, 2010.

[148] H.E. Merritt. Hydraulic Control Systems. John Wiley \& Sons Inc, 1967.

[149] L. Cheng. Development of an Endoscopic Irrigation Pump: Experience with Byrne Medical, Inc. PhD thesis, Texas A\&M University, 2007.

[150] Kwang W. Oh and Chong H. Ahn. A review of microvalves. Journal of Micromechanics and Microengineering, 16(5):R13-R39, March 2006.

[151] J. Ueda, T.W. Secord, and H.H. Asada. Large effective-strain piezoelectric actuators using nested cellular architecture with exponential strain amplification mechanisms. IEEE/ASME Transactions on Mechatronics, 15(5):770$782,2010$.

[152] J.-J. Tzen, S.-L. Jeng, and W.-H. Chieng. Modeling of piezoelectric actuator for compensation and controller design. Precision Engineering, 27(1):70-86, 2003.

[153] M. Richmond. Solenoids and magnetic fields. http://spiff.rit.edu/ classes/phys313/lectures/sol/sol_f01_long.html. Accessed: October 2011.

[154] R. Peikert, D. Würtz, M. Monagan, and C. de Groot. Packing circles in a square: A review and new results. System Modelling and Optimization, pages 45-54, 1992.

[155] N.J.A. Sloane. Table of maximal density of a packing of equal spheres in n-dimensional euclidean space (for $\mathrm{n}, 3$ the values are only conjectural). http://oeis.org/A093825/a093825.txt. Accessed: March 2013.

[156] F. Incropera and D. DeWitt. Introduction to heat transfer. John Wiley and Sons Inc., 1985.

[157] M.H. Sharqawy, J.H. Lienhard, and S.M. Zubair. Thermophysical properties of seawater: A review of existing correlations and data. Desalination and Water Treatment, 16(1-3):354-380, 2010.

[158] F. Kreith. The CRC handbook of thermal engineering. Springer, 2000.

[159] V. Ochkov. Thermal conductivity of seawater and its concentrates. http://twtmas.mpei.ac.ru/mas/Worksheets/HEDH/ Tab-5-5-13-2-Ther-Cond-Seawater.mcd. Accessed: June 2012. 
[160] G.F. Hewitt. Heat exchanger design handbook. Begelle House Inc., 1998.

[161] MatWeb. Overview of materials for stainless steel. http://matweb.com/search/DataSheet.aspx?MatGUID= 71396e57ff5940b791ece120e4d563e0. Accessed: November 2012.

[162] Alan E Mikesky, Robert Topp, Janet K Wigglesworth, David M Harsha, and Jeffrey E Edwards. Efficacy of a home-based training program for older adults using elastic tubing. European journal of applied physiology and occupational physiology, 69(4):316-320, 1994.

[163] D.R. Berg. Hydraulic dexterous manipulator for minimally invasive surgery: CAD model. http://dx.doi.org/10.6084/m9.figshare.810033, 2013. Accessed: September 2013.

[164] A.N. Gent. Elastic instabilities in rubber. International Journal of NonLinear Mechanics, 40(2):165-175, 2005.

[165] M. Kroh, K. El-Hayek, S. Rosenblatt, B. Chand, P. Escobar, J. Kaouk, and S. Chalikonda. First human surgery with a novel single-port robotic system: cholecystectomy using the da vinci single-site platform. Surgical Endoscopy, 25(11):3566-3573, 2011.

[166] A.M. Okamura. Methods for haptic feedback in teleoperated robot-assisted surgery. Industrial Robot: An International Journal, 31(6):499-508, 2004.

[167] R. Richa, M. Balicki, E. Meisner, R. Sznitman, R.H. Taylor, and G.D. Hager. Visual tracking of surgical tools for proximity detection in retinal surgery. In Information Processing in Computer Assisted Interventions, volume 6689, pages 55-66, 2011.

[168] C.E. Reiley, T. Akinbiyi, D. Burschka, D.C. Chang, A.M. Okamura, and D.D. Yuh. Effects of visual force feedback on robot-assisted surgical task performance. The Journal of thoracic and cardiovascular surgery, 135(1):196202, 2008.

[169] A. Krieger, R.C. Susil, C. Ménard, J.A. Coleman, G. Fichtinger, E. Atalar, and L.L. Whitcomb. Design of a novel mri compatible manipulator for image guided prostate interventions. IEEE Transactions on Biomedical Engineering, 52(2):306-313, 2005.

[170] P.Y. Li. Design and control of a hydraulic human power amplifier. In Proceedings of the 2004 ASME International Mechanical Engineering Congress Exposition, pages 385-393, Anaheim, CA, 2004. ASME. 
[171] H. Kazerooni. The human power amplifier technology at the University of California, Berkeley. Robotics and autonomous systems, 19(2):179-187, 1996.

[172] R.H. Taylor and D. Stoianovici. Medical robotics in computer-integrated surgery. IEEE Transactions on Robotics and Automation, 19(5):765-781, 2003.

[173] Y. Kobayashi, T. Hoshi, K. Kawamura, and M.G. Fujie. Control method for surgical robot to prevent overload at vulnerable tissue. In IEEE International Conference on Robotics and Automation, pages 1893-1899. IEEE, 2007.

[174] B. Yao, F. Bu, J. Reedy, and G.T.C. Chiu. Adaptive robust motion control of single-rod hydraulic actuators: theory and experiments. IEEE/ASME Transactions on Mechatronics, 5(1):79-91, 2000.

[175] R. Legtenberg, A.W. Groeneveld, and M. Elwenspoek. Comb-drive actuators for large displacements. Journal of Micromechanics and Microengineering, 6:320-329, 1996.

[176] C. Chen. Design and modeling for comb drive actuator with enlarged static displacement. Sensors and Actuators A: Physical, 115(2-3):530-539, 2004.

[177] J. D. Grade, H. Jerman, and T. W. Kenny. Design of large deflection electrostatic actuators. Journal of Microelectromechanical Systems, 12(3):335-343, 2003.

[178] S. Schonhardt, J. Korvink, J. Mohr, U. Hollenbach, and U. Wallrabe. Optimization of an electromagnetic comb drive actuator. Sensors and Actuators A: Physical, 154:212-217, 2009.

[179] G. Zhou and P. Dowd. Tilted folded-beam suspension for extending the stable travel range of comb-drive actuators. Journal of Micromechanics and Microengineering, 13(2):178-183, 2003.

[180] B. Borovic, F. L. Lewis, A. Q. Liu, E. S. Kolesar, and D. Popa. The lateral instability problem in electrostatic comb drive actuators: modeling and feedback control. Journal of Micromechanics and Microengineering, 16(7):12331241, 2006.

[181] W. Huang and G. Lu. Analysis of lateral instability of in-plane comb drive mems actuators based on a two-dimensional model. Sensors and Actuators A: Physical, 113(1):78-85, 2004. 
[182] D. Kamiya, T. Hayama, and M. Horie. Electrostatic comb-drive actuators made of polyimide for actuating micromotion convert mechanisms. $\mathrm{Mi}$ crosystem Technologies, 5(4):161-165, 1999.

[183] Nanofabrication Center. NFC equipment and process list. http://www. nfc.umn.edu/equipment/. Accessed: June 2009.

[184] Y. Zhao and T. Cui. Fabrication of high-aspect-ratio polymer-based electrostatic comb drives using the hot embossing technique. Journal of $\mathrm{Mi}$ cromechanics and Microengineering, 13:430-435, 2003. 


\section{Appendix A}

\section{Design Alternatives}

\section{Manipulator Backbone}

\section{Spherical Joint Structure}

One possible alternative solution to the continuum beam backbone that has been explored is a jointed structure as shown in Fig. A.1, which would be driven by an array of tensile wires (three or four) that run along the outer edge of the joined links. The jointed structure provides an option that is more easily modeled as the tip position can be predicted by knowing the orientation of a small number of segments. Further, the use of rigid links means that the resulting manipulator will be more precise than a similar continuum beam based manipulator since each there are a finite number of degrees of freedom that can each be actuated. The primary disadvantage of this approach is that the resulting structure is more complicated and thus more difficult to manufacture. 


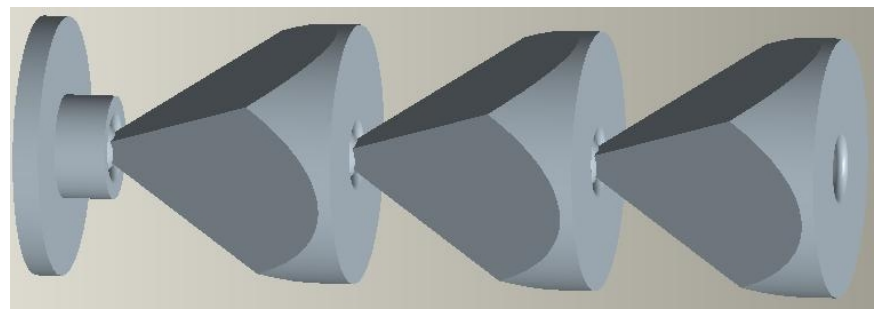

Figure A.1: Jointed support structure.

\section{Analytical Description}

This jointed structure is comprised of multiple independent pieces connected through spherical joints, which were chosen in order to provide minimal resistance against manipulation and rotational freedom in all directions. It was necessary to develop an analytical description of the proposed component in order to estimate how the device might perform more accurately than can be done by intuition. For the purposes of developing the analytical model for this structure, each piece was treated as an individual free body. First, it is necessary to define the geometric properties of the of each link and the relationship between the links and the tensile wire as shown in Fig. A.2. The geometry of each free body at the location of the spherical joint is assumed to be a point for simplification. 


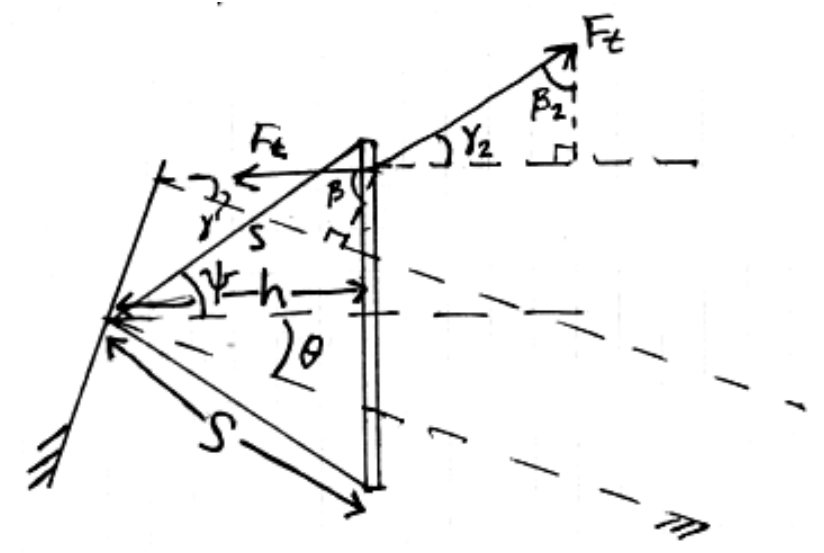

Figure A.2: Sketch showing the labeled geometric references used in later equations.

As shown here, $\psi$ is the angle between the edge and central axis of a link, $\theta$ is the angle between a line perpendicular to the base and the link's central axis, $\gamma$ is the angle between a line perpendicular to the base and the force vector of the tensile wire in the proximal direction, and $\beta$ is the angle between a line parallel to the base and the force vector of the tensile wire in the proximal direction. Further, $F_{t}$ is the force applied to the tensile wire, $h$ is the central axis height of the link, and $s$ is the side length of the link. Then, by geometry it can be shown that the angle $\gamma$ can be derived as,

$$
\gamma_{i}=\tan ^{-1}\left(\frac{\sin \left(\theta_{i}+\psi\right)-\sin \psi}{\cos \left(\theta_{i}+\psi\right)}\right)
$$

and also that

$$
\beta_{i}=\frac{\pi}{2}-\gamma_{i}
$$

where the subscript $i$ denotes that the variable can be defined as such for any of the links within the joint.

Using this notation, a free body diagram of an example three-link joint (Fig. A.3) can be used to derive the moment and force balance equations that allow the 
joint to be fully defined in space for a given tensile force. For simplification of the model, the mass of the individual components as well as any frictional losses in the spherical joints or tensile wire interactions is assumed negligible. Further, the interactions between the tensile wire and the free bodies are assumed to occur as a point location and in only one direction.

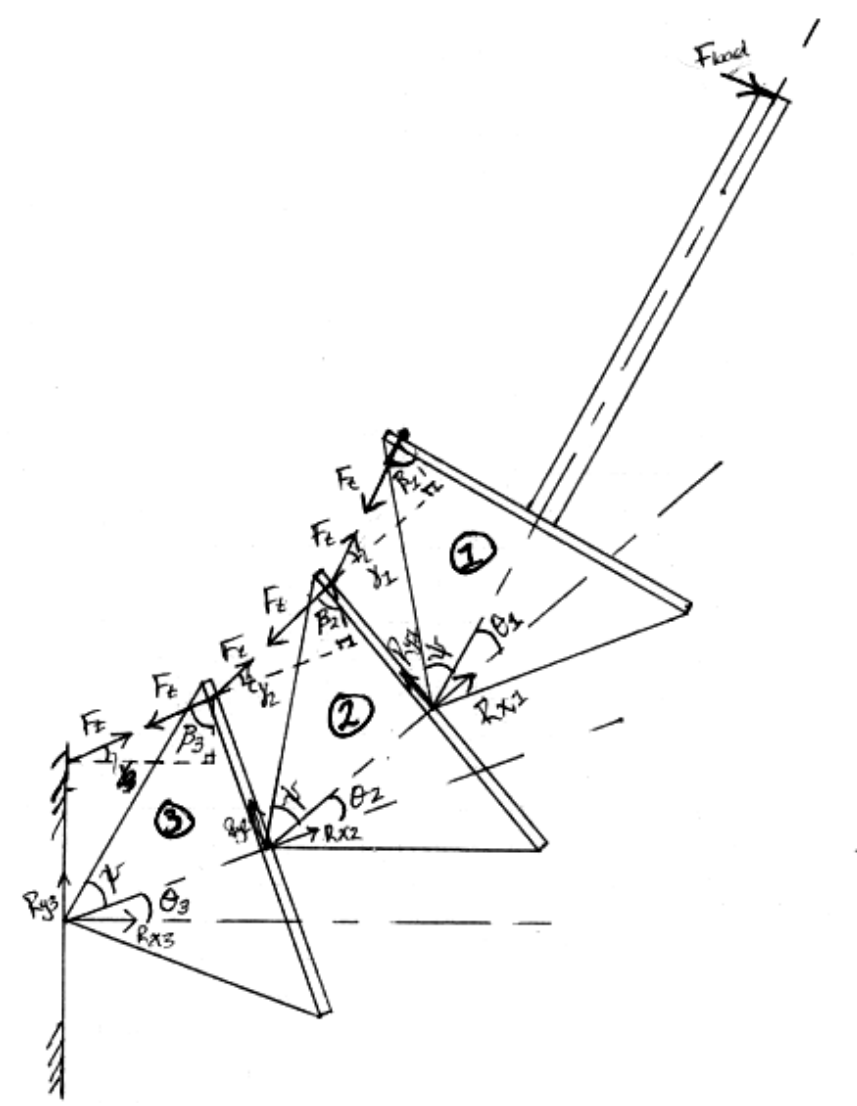

Figure A.3: Free body diagram of a sample three-link joint.

For the distal link, labeled 1 in Fig. A.3, the balance equations are realized as,

$$
\begin{aligned}
\sum M=0= & F_{t} \sin \beta_{1} s \sin \left(\psi+\theta_{1}\right)-F_{t} \cos \beta_{1} s \cos \left(\psi+\theta_{1}\right) \\
& -F_{\text {load }} \sin \theta_{1} l \sin \theta_{1}-F_{\text {load }} \cos \theta_{1} \cos \theta_{1}
\end{aligned}
$$




$$
\begin{aligned}
& \sum F_{x, 1}=0=R_{x, 1}-F_{t} \sin \beta_{1}+F_{\text {load }} \sin \theta_{1} \\
& \sum F_{y, 1}=0=R_{y, 1}-F_{t} \cos \beta_{1}-F_{\text {load }} \cos \theta_{1} .
\end{aligned}
$$

Additionally, for the remaining two elements, the balance equations are,

$$
\begin{aligned}
\sum M_{i}=0= & F_{t} \sin \beta_{i} s \sin \left(\psi+\theta_{i}\right)-F_{t} \cos \beta_{i} s \cos \left(\psi+\theta_{i}\right) \\
& -F_{t} \cos \left(\gamma_{i-1}+\theta_{i}\right) s \sin \left(\psi+\theta_{i}\right)+F_{t} \sin \left(\gamma_{i-1}+\theta_{i}\right) s \cos \left(\psi+\theta_{i}\right) \\
& -R_{y, i-1} \sin \theta_{i} h \sin \theta_{i}-R_{y, i-1} \cos \theta_{i} h \cos \theta_{i} \\
& +R_{x, i-1} \cos \theta_{i} h \sin \theta_{i}-R_{x, i-1} \sin \theta_{i} h \cos \theta_{i} \\
\sum F_{x, i}=0= & R_{x, i}-F_{t} \sin \beta_{i}+F_{t} \cos \left(\gamma_{i-1}+\theta_{i}\right)+R_{y, i-1} \sin \theta_{i}-R_{x, i-1} \sin \theta_{i} \\
\sum F_{y, i}=0= & R_{y, i}-F_{t} \cos \beta_{i}+F_{t} \sin \left(\gamma_{i-1}+\theta_{i}\right)-R_{y, i-1} \cos \theta_{i}-R_{x, i-1} \sin \theta_{i},
\end{aligned}
$$

where $i=2,3$ corresponding the the elements labeled 2 and 3 in Fig. A.3.

\section{Conclusions}

Solving these equations simultaneously allows the motion of the jointed structure to be predicted for a given load condition and control inputs. Ultimately, the model suggests that despite the advantages that this design presents in terms of freedom of motion, it may not provide sufficient control over tip position for the application. In particular, the proximal link in the series actually deviates in the opposite direction from the applied tension until the following link reaches the limit of its rotational motion as demonstrated using the prototype in Fig. A.4. This situation could be avoided by having low mass segments and applying a slight pre-tension to the wires. One additional difficulty for the realization of this design would be find a robust method of fabricating the joints such that they could withstand repeated use reliably at the appropriate scale. 


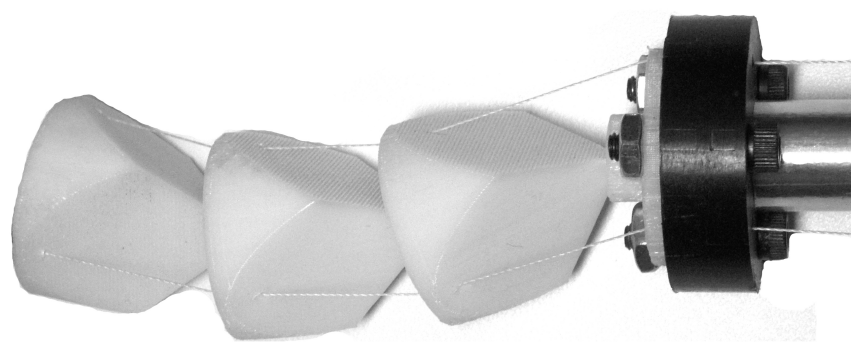

Figure A.4: Demonstration of problematic joint response.

\section{Control Valve}

\section{MEMS Valve}

As an alternative solution to the valve design presented in Section 5 , a review of the common micro-fluidic valve solutions was conducted and no existing valve design was found suitable for the present application due to the limitations of high supply pressure, proportional control, and method of activation. Therefore, the feasibility of existing MEMS technologies to the application of proportional flow control was investigated. In order to successfully regulate flow pressures greater than a few thousand pascals, it is necessary to design for pressure balancing, an example of which is shown in Fig. A.5, due to the low actuation forces that can be produced by most microelectromechanical systems (MEMS) based applications [142]. Further, it is often difficult to achieve large displacements, such as those that would be necessary to achieve the desired flow rates, using MEMS techniques. 


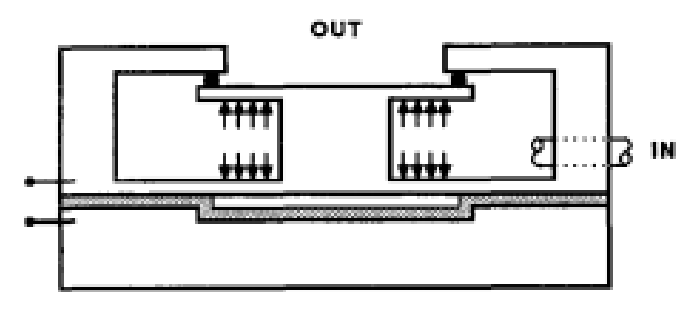

valve closed

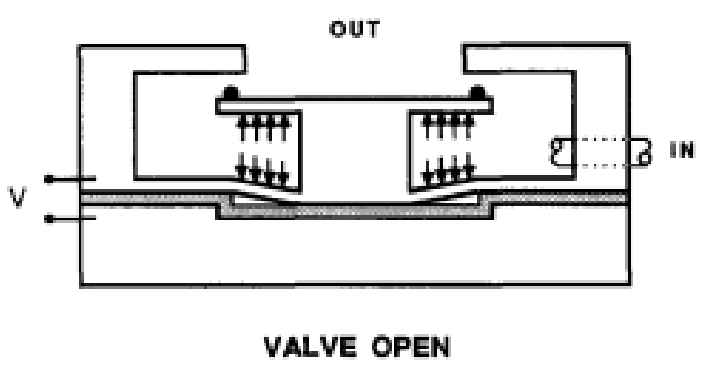

Figure A.5: Example of a pressure balanced valve concept [142].

One attractive solution in this space is the electrostatic comb-drive actuator, which has been shown in the past to be capable of large displacements relative to actuator size [175]. It is necessary to obtain large scale actuator displacements in order to achieve a sufficient valve orifice size for the desired fluid flow rate. For the designed system, a maximum displacement of $60 \mu \mathrm{m}$ would be sufficient; however, greater displacement capability would permit a lower orifice aspect ratio which would yield better flow characteristics.

A review of the literature reveals several electrostatic comb-drive actuator designs intended to extend the range over which the actuator is capable of operating [176, 177, 178, 179]. Each of these solutions have inherent advantages and disadvantages. However, of primary importance in the design of large displacement electrostatic actuators is the balance of displacement capability and lateral instability, sometimes referred to side pull-in [180]. Side pull-in occurs when the applied voltage difference across the comb-drive electrodes is large enough that 
the electrostatic force between the interdigitated fingers causes them to pull together, thus disabling the actuator [181]. To combat this effect, the comb-drive actuator must be designed such that the lateral stiffness of the suspension system is sufficiently high to avoid pull-in over the operating range of the actuator.

\section{Comb-Drive Principles}

One of the key components of the design for a novel microfluidic control valve is the method for achieving large displacements. In the present design, the method chosen for producing the mechanism displacement is use of an electrostatic combdrive actuator. The comb-drive actuator achieves motion through the application of a voltage difference across fixed and movable interdigitated comb fingers. This voltage difference produces an electrostatic field between the neighboring fingers and thus induces an attractive force that can be modeled as a parallel plate arrangement, see Fig. A.6 [175, 182].

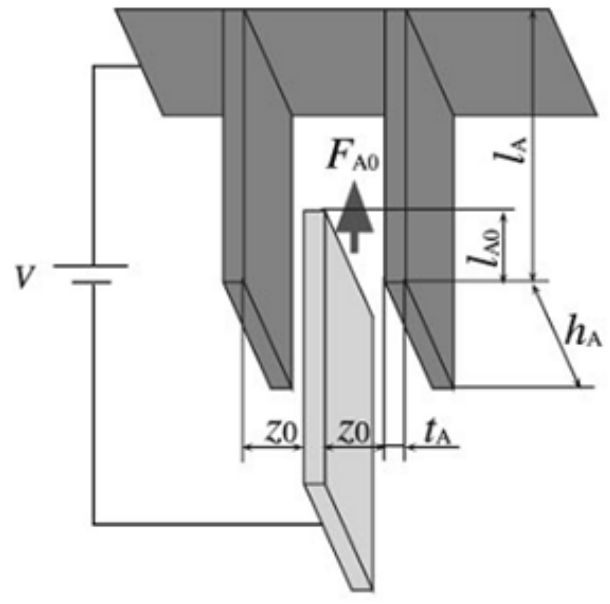

Figure A.6: Geometry of a single set of comb-drive fingers [182]. 
Using this parallel plate model, with the geometry shown in Fig. A.6, the capacitance of parallel plates can be expressed as

$$
C=\frac{2 n \epsilon_{0} h_{A} l_{A 0}}{z_{0}}
$$

where $n$ is the number of fingers and $\epsilon_{0}$ is dielectric constant in free air. From this, the force, $F_{A 0}$, can be described as presented in the literature [175, 182];

$$
F_{A 0}=\frac{1}{2} \frac{\delta C}{\delta y} V^{2}=\frac{n \epsilon_{0} h_{A}}{z_{0}} V^{2}
$$

Concurrent to this, it is necessary to model the effect of the spring used to return the actuator to its initial position when the voltage difference across the combdrive fingers is removed. One design for the spring suspension system is the folded flexure design shown in Fig. A.7 [177].

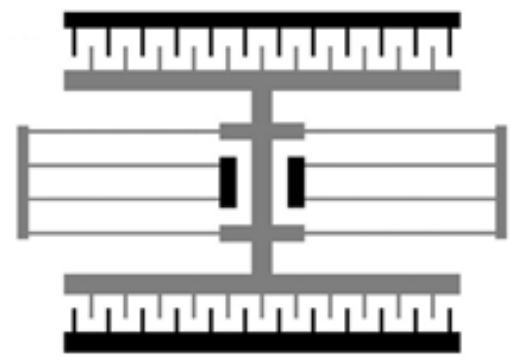

Figure A.7: Folded flexure design commonly used for large displacement electrostatic comb-drive actuators [177].

The force that must be applied to the spring to achieve the desired deflection can be computed by modeling the individual springs as beam elements with fixed ends. Then the force of the spring is modeled as presented in the literature [179]; 


$$
F_{\text {spring }}=\delta \frac{24 E I}{l^{3}}=2 \delta \frac{E h_{A} t_{S}^{3}}{l^{3}} .
$$

By setting the actuator force equal to the spring force, it is possible to design the electrostatic actuator such that the desired displacement can be achieved. The parameters that are able to be designed are the number of fingers, $n$, the spring length, $l$, the spring width, $t_{S}$, the structural thickness, $h_{A}$, the finger gap, $z_{0}$, and the operating voltage, $V$.

The scaling effects can be particularly significant and thus should be considered during the design process. For example, the force necessary to drive a fixed-fixed beam spring as proposed in the present project is greatly subjective to the effects of geometric scaling. As the overall size of the beam suspension system is scaled down by an order of magnitude from the original size, the force necessary to achieve the new scaled displacement reduced by two orders of magnitude.

This effect is beneficial since it allows the electrostatic actuator requirement to be greatly reduced for even small changes in the suspension system size. One possible realization of this is in terms of the driving voltage for the electrostatic actuator. Assuming consistent geometry of the actuator, a smaller requirement in force would mean that the driving voltage could be reduced, thus resulting in a more efficient device. Additionally, it is interesting to note that the force output from the actuator is independent of geometry scaling assuming that the finger gap scales proportionally to structural thickness of the actuator fingers. Therefore, the limitation of geometric scaling of the actuator is only limited by the constraints of the fabrication process used to produce the device.

Based upon these principles, a valve design was developed that would be operated by an electrostatic comb-drive actuator. The design included $100 \mu \mathrm{m}$ long fingers in order to achieve a desired translation of at least $60 \mu \mathrm{m}$. Additionally, the orifice of the valve was sized at $50 \mu \mathrm{m}$ by $200 \mu \mathrm{m}$ in order to achieve a flow rate 
of $9 \mathrm{~mL} / \mathrm{min}$. The layout shown in Fig. A.8 represents the three layer masks necessary to manufacture this valve design. The green background represents the silicon substrate upon which the valve is constructed. The yellow layer locates the positions of the valve orifices and thus represents the area which will be etched away from the silicon wafer. The blue layer shows the structure of the valve itself, which is produced from polysilicon. Finally, the red layer represents the anchor which allows the polysilicon to adhere to the wafer below. The details of this process are described in the following section.

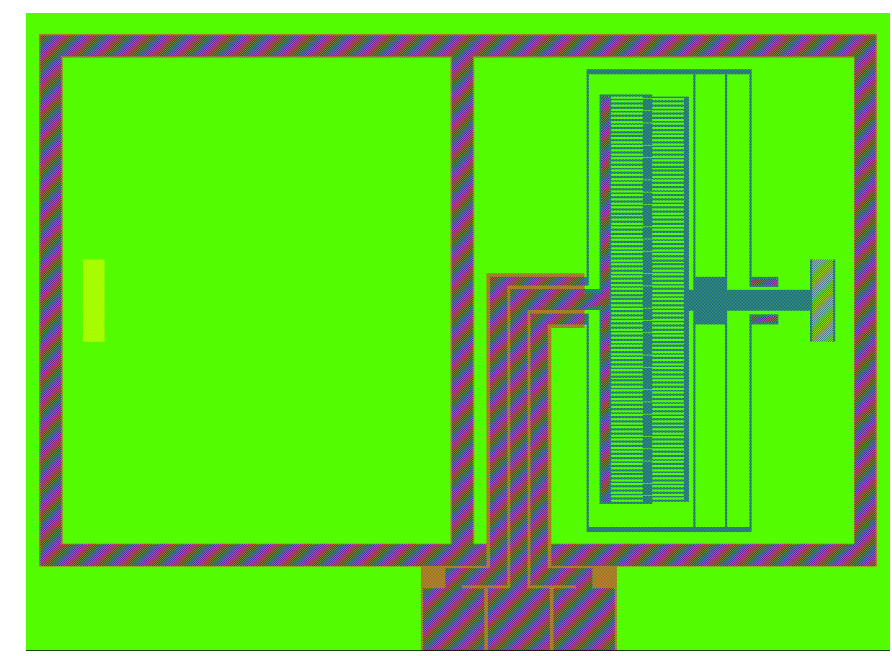

Figure A.8: Mask layout for valve design.

\section{Processing}

A possible fabrication process is presented in the following flowchart. To accompany each of the steps, a cross sectional model of the wafer throughout the fabrication process is presented alongside each description. Information regarding specific deposition/etch rates and recipes to be used have been adapted from the website of the Nanofabrication Center at the University of Minnesota - Twin Cities [183]. For convenience, a key correlating the different cross-hatching patterns used to the appropriate material is shown below in Fig. A.9. 


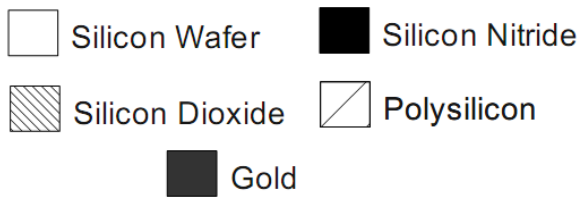

Figure A.9: Key to be used to identify materials in the process design figures.

1. Start with a bare $\langle 100\rangle$ silicon wafer.

(a) Wafer should be cleaned using the RCA clean procedure.

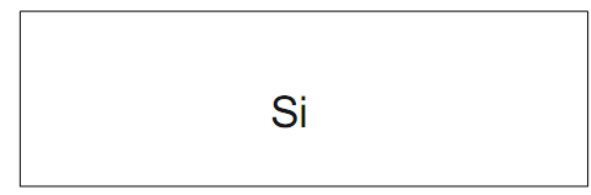

Figure A.10: Bare silicon wafer.

2. Deposit $2000 \AA$ of silicon nitride by LPCVD.

(a) Deposition for 60 min. using the default Low Stress Nitride recipe.

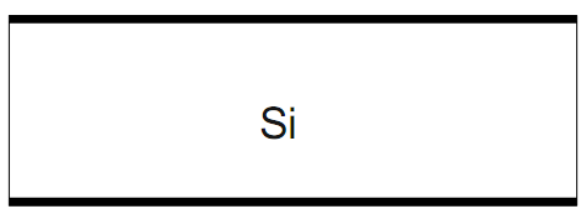

Figure A.11: Wafer after silicon nitride deposition.

3. Deposit $1 \mu \mathrm{m}$ of silicon dioxide by LPCVD.

(a) Deposition for 125 min. using the PSG recipe.

(b) Anneal the wafer. 


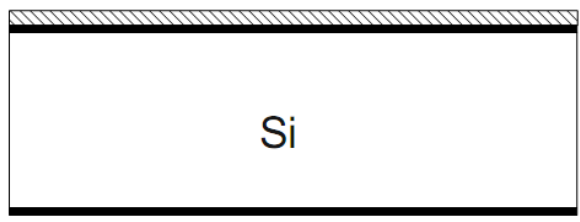

Figure A.12: Wafer after silicon dioxide deposition.

4. Perform photolithography on silicon dioxide.

(a) Deposit photoresist (PR-1813) by spinning.

(b) Expose photoresist to UV using mask (dark field).

(c) Etch photoresist using photoresist developer.

(d) Etch unprotected Silicon Dioxide using HF etch.

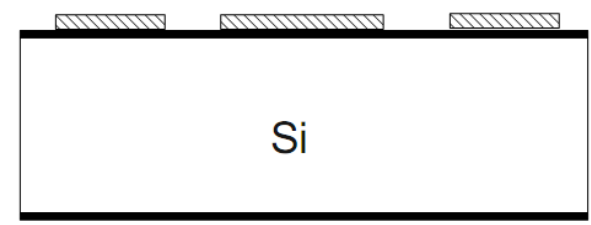

Figure A.13: Wafer after silicon dioxide etching.

5. Deposit polysilicon by LPCVD.

(a) Deposition for 7 hours using a combined doped/undoped Polysilicon recipe.

(b) Anneal the wafer to reduce the chance of undesired deformation.

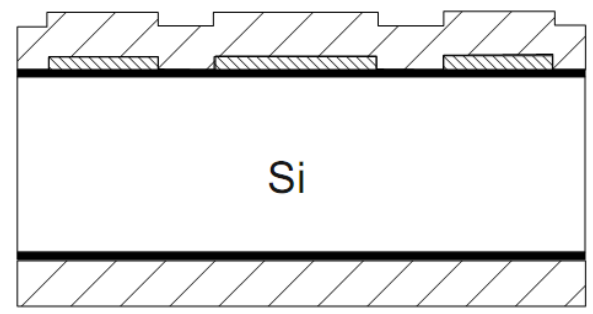

Figure A.14: Wafer after polysilicon deposition. 
6. Perform photolithography on polysilicon.

(a) Deposit photoresist (PR 1813) by spinning.

(b) Expose photoresist to UV using mask (light field).

(c) Etch photoresist using photoresist developer.

(d) Etch unprotected Polysilicon using RIE.

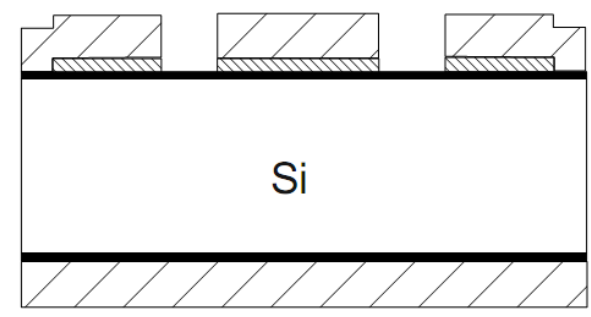

Figure A.15: Wafer after polysilicon etching.

7. Deposition of a reflective gold layer by electron-beam evaporation.

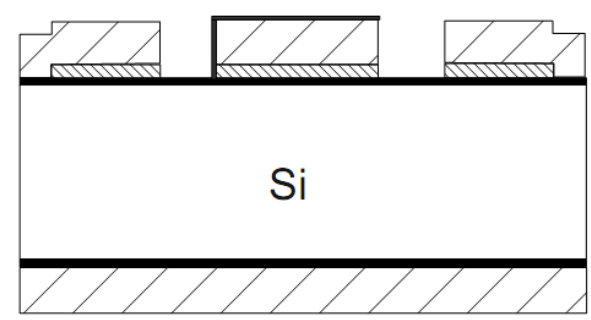

Figure A.16: Wafer after gold deposition.

8. Remove sacrificial silicon dioxide by HF etch.

(a) Wafer will need to go into the Critical Point Dryer to remove any remaining etchants. 


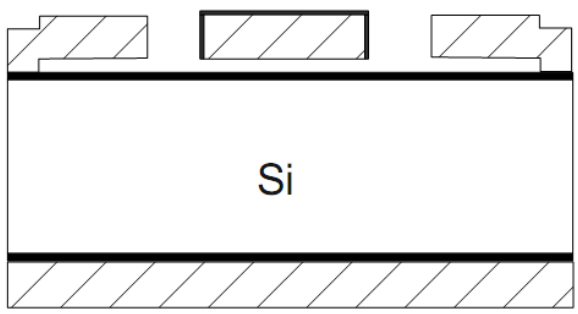

Figure A.17: Wafer after removal of sacrificial layer.

9. Wafer diced into individual units and assembled with packaging to form final product.

By following this procedure through step six, a prototype of this design was produced and is shown in Fig. A.18.

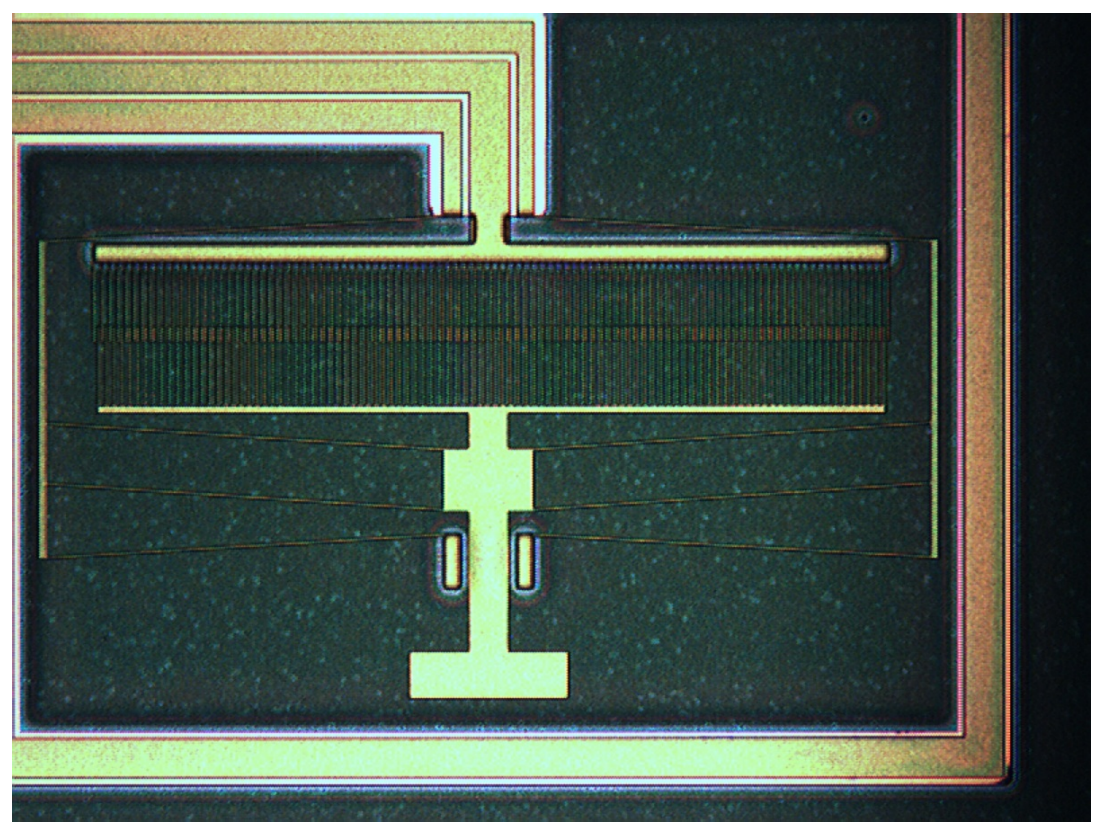

Figure A.18: Valve design prototype shown at 5x magnification. 


\section{Valve Design Considerations}

Experimentation with MEMS processing using the above procedure revealed that there are some limitations of the methodology that need to be given greater consideration. Primarily, the use of a $1 \mu \mathrm{m}$ sacrificial silicon dioxide layer was found to be insufficient and led to high stiction susceptibility. This issue may be remedied by increasing the sacrificial layer thickness to $2 \mu \mathrm{m}$. Further, by analyzing similar processing procedures described in the literature, it may be necessary to increase the thickness of the structural polysilicon layer [175]; however, the method by which this can be achieved has yet to be determined. Other avenues of fabrication have been presented in the literature and shown to produce high-aspect-ratio comb-drive structures [184]. 


\section{Appendix B}

\section{IFM Code}

\section{Two-Dimensional IFM Code}

The following code is written in Matlab M-code. This code accepts input beam end conditions and computes the most likely beam shape and end loads for the two-dimensional planar case.

1 function [moment_L,forceZ,forceX,x] = energyMethod_standalone( thetaL, $\mathrm{zL}, \mathrm{xL}, \mathrm{x} 0)$

$2 \%$ This function returns the potential energy in the beam, the moment produced at the tip, and the force produced at the tip for some given input tip location and orientation. The solution is found by minimizing the potential energy in the beam to find the optimal ( most likely) beam shape.

3

$4 \%$ Input beam material and geometric properties.

${ }_{5} \mathrm{E}=28 \mathrm{e} 9 ;$ 


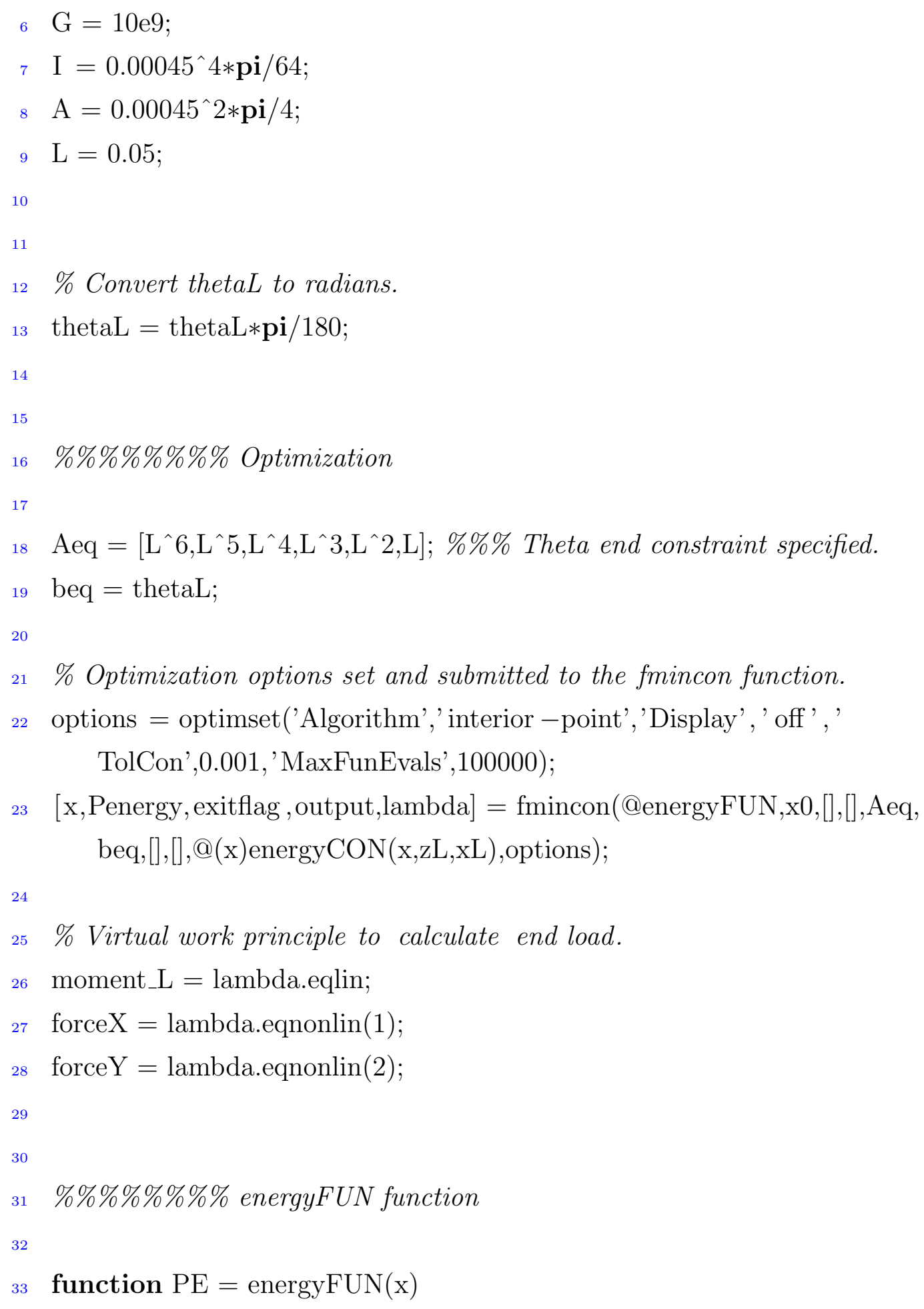$$
\text { (1) }
$$ 
34

$\%$ This function returns the potential energy of the beam for a provided set of input parameters.

36

37

38

39

40

41

42

43

$44 \% \% \% \% \% \%$ Parameterization of Theta

$45 \mathrm{~s}=0: \mathrm{L} / 100: \mathrm{L}$;

46

47

48

49

50

51

52

53

54

55

56

57

58

59

60

61

62

$\%$ Input beam material and geometric properties.

$\mathrm{E}=28 \mathrm{e} 9$

$\mathrm{G}=10 \mathrm{e} 9$;

$\mathrm{I}=0.00045^{\wedge} 4 * \mathrm{pi} / 64$

$\mathrm{A}=0.00045^{\wedge} 2 * \mathrm{pi} / 4$;

$\mathrm{L}=0.05$

.

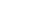

$\%$ Derivative of theta with respect to $s$.

dtheta $=\mathrm{x}(1: 6) *\left[6 * \mathrm{~s} .{ }^{\wedge} 5 ; 5 * \mathrm{~s} . \wedge 4 ; 4 * \mathrm{~s} .{ }^{\wedge} 3 ; 3 * \mathrm{~s} .{ }^{\wedge} 2 ; 2 * \mathrm{~s} ; \operatorname{ones}(1,101)\right]$;

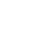

$\%$ Potential energy function.

integrand $=\mathrm{E} * \mathrm{I} *$ dtheta. ${ }^{\wedge} 2$;

$\mathrm{PE}=0.5 * \operatorname{trapz}(\mathrm{s}$, integrand $)$

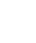

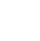

$\% \% \% \% \% \% \% \%$ energyCON function

(1)

function $[\mathrm{c}, \mathrm{ceq}]=\operatorname{energyCON}(\mathrm{x}, \mathrm{zL}, \mathrm{xL})$

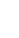

$\mathrm{c}=[]$ 


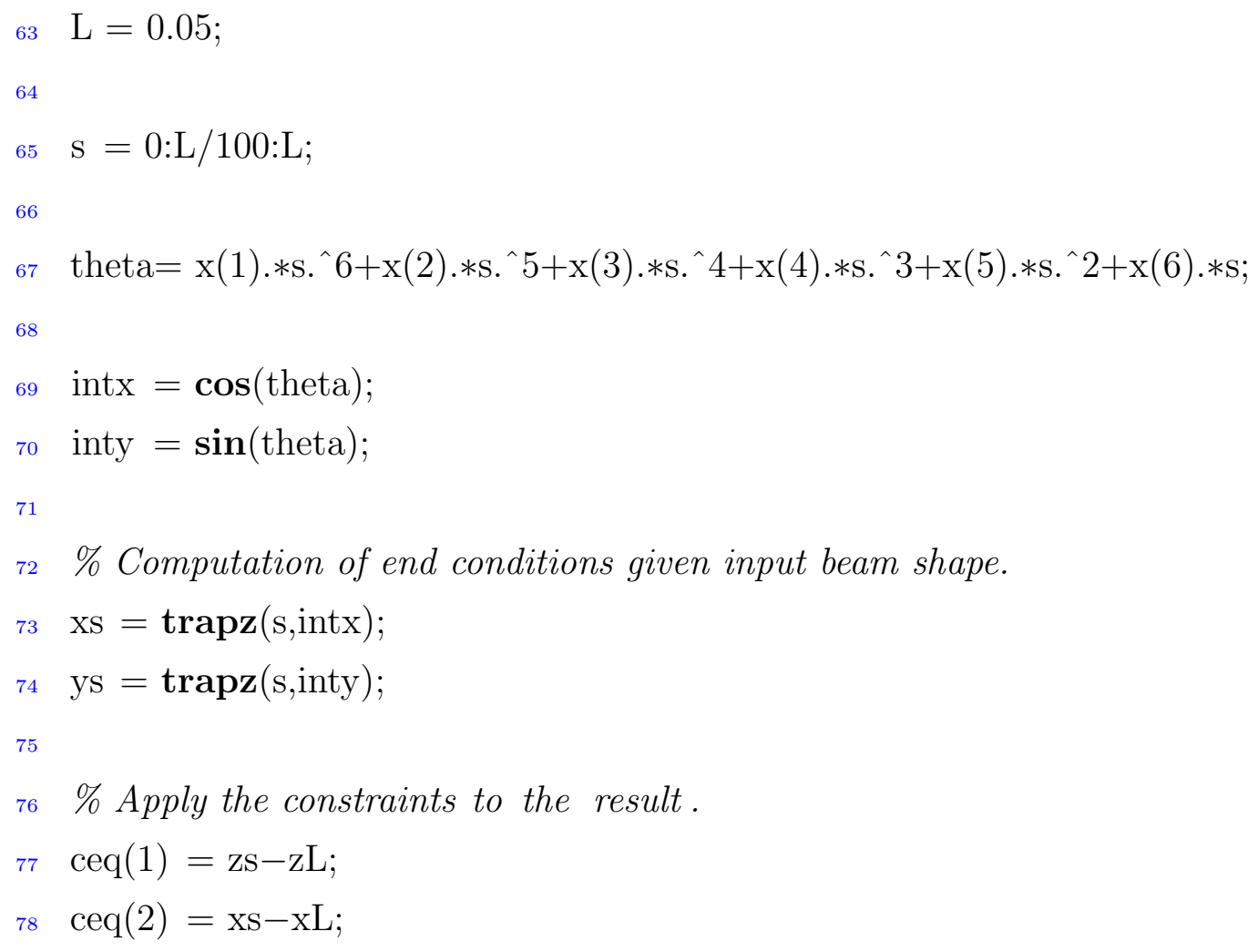

\section{Three-Dimensional IFM Code}

The following code is written in Matlab M-code. This code accepts input beam end conditions and computes the most likely beam shape and end loads for the three-dimensional planar case.

1 function [moment_phi,moment_theta,moment_psi,forceX,forceY,forceZ] $=$ energyMethod_standalone3D(phiL,thetaL,psiL, $\mathrm{xL}, \mathrm{yL}, \mathrm{zL})$ 
${ }_{2} \%$ This function returns the potential energy in the beam, the moment produced at the tip, and the force produced at the tip for some given input tip location and orientation. The solution is found by minimizing the potential energy in the beam to find the optimal ( most likely) beam shape.

3

$4 \%$ Input beam material and geometric properties.

$5 \quad \mathrm{E}=28 \mathrm{e} 9$;

$6 \mathrm{G}=10 \mathrm{e} 9$;

$7 \quad \mathrm{I}=0.00045^{\wedge} 4 * \mathbf{p i} / 64$;

$8 \mathrm{~A}=0.00045^{\wedge} 2 * \mathrm{pi} / 4$;

$9 \quad \mathrm{~L}=0.05$;

10

$1 \mathrm{x} 0=[0,0,0,0,0,0,0,0,0,0,0,0,0,0,0,0,0,0] ;$

12

$13 \%$ Convert angles to radians.

14 thetaL $=$ thetaL $* \mathbf{p i} / 180$;

15 phiL $=$ phiL $* \mathbf{p i} / 180$;

16

psiL $=$ psiL $* \mathbf{p i} / 180$;

17

18

$\% \% \% \% \% \% \% \%$ Optimization

20

Aeq $=\left[\mathrm{L}^{\wedge} 6, \mathrm{~L}^{\wedge} 5, \mathrm{~L}^{\wedge} 4, \mathrm{~L}^{\wedge} 3, \mathrm{~L}^{\wedge} 2, \mathrm{~L}, 0,0,0,0,0,0,0,0,0,0,0,0 ;\right.$ $0,0,0,0,0,0, \mathrm{~L}^{\wedge} 6, \mathrm{~L}^{\wedge} 5, \mathrm{~L}^{\wedge} 4, \mathrm{~L}^{\wedge} 3, \mathrm{~L}^{\wedge} 2, \mathrm{~L}, 0,0,0,0,0,0$; $\left.0,0,0,0,0,0,0,0,0,0,0,0, \quad \mathrm{~L}^{\wedge} 6, \mathrm{~L}^{\wedge} 5, \mathrm{~L}^{\wedge} 4, \mathrm{~L}^{\wedge} 3, \mathrm{~L}^{\wedge} 2, \mathrm{~L}\right] ; \% \% \%$ phi, theta, and psi end constraint specified.

24 beq $=[$ phiL;thetaL;psiL];

25

${ }_{26} \%$ Optimization options set and submitted to the fmincon function. 
27

28

30

31

32

33

34

35

36

37

38

39

41

42

43

45

46

47

48

49

50

51

52

53

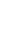

(1)

options $=$ optimset('Algorithm','interior - point','TolCon',0.001,'

MaxFunEvals',100000);

$[\mathrm{x}$, Penergy, exitflag, output,lambda $]=$ fmincon $(@$ energyFUN,x0,[], [],Aeq,

beq,[],[],@(x)energyCON(x,xL,yL,zL,thetaL,phiL,psiL),options);

$\%$ Virtual work principle to calculate end load.

moment_phi = lambda.eqlin(1);

moment_theta $=$ lambda.eqlin $(2)$;

moment_psi = lambda.eqlin(3);

forceX = lambda.eqnonlin(1);

forceY = lambda.eqnonlin(2);

forceZ = lambda.eqnonlin(3);

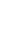

\section{$\% \% \% \% \% \% \%$ energyFUN function}

.

function $\mathrm{PE}=$ energyFUN $(\mathrm{x})$

$\%$ This function returns the potential energy of the beam for a provided set of input parameters.

$\%$ Input beam material and geometric properties.

$\mathrm{E}=28 \mathrm{e} 9$

$\mathrm{G}=10 \mathrm{e} 9$

$\mathrm{I}=0.00045^{\wedge} 4 * \mathbf{p i} / 64$

$\mathrm{A}=0.00045^{\wedge} 2 * \mathbf{p i} / 4$

$\mathrm{L}=0.05$; $\% \% \% \% \% \% \% \%$ Parameterization of Phi, Theta, and Psi 
54

55

56

57

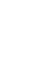

58

59

60

61

62

63

65

66

67

68

69

70

71

72

73

74

$75 \mathrm{~S}=0: \mathrm{L} / 100: \mathrm{L}$

76

77 phi $=\mathrm{x}(1) \cdot * \mathrm{~s} .{ }^{\wedge} 6+\mathrm{x}(2) . * \mathrm{~s} .{ }^{\wedge} 5+\mathrm{x}(3) \cdot * \mathrm{~s} .{ }^{\wedge} 4+\mathrm{x}(4) \cdot * \mathrm{~s} .{ }^{\wedge} 3+\mathrm{x}(5) \cdot * \mathrm{~s} .{ }^{\wedge} 2+\mathrm{x}(6) \cdot * \mathrm{~s}$;

78 theta $=\mathrm{x}(7) \cdot * \mathrm{~s} .{ }^{\wedge} 6+\mathrm{x}(8) \cdot * \mathrm{~s} .{ }^{\wedge} 5+\mathrm{x}(9) \cdot * \mathrm{~s} .{ }^{\wedge} 4+\mathrm{x}(10) \cdot * \mathrm{~s} .{ }^{\wedge} 3+\mathrm{x}(11) \cdot * \mathrm{~s} .{ }^{\wedge} 2+\mathrm{x}$

$(12) . * \mathrm{~s} ;$

79

$\mathrm{psi}=\mathrm{x}(13) . * \mathrm{~s} .{ }^{\wedge} 6+\mathrm{x}(14) . * \mathrm{~s} .{ }^{\wedge} 5+\mathrm{x}(15) . * \mathrm{~s} .{ }^{\wedge} 4+\mathrm{x}(16) . * \mathrm{~s} .{ }^{\wedge} 3+\mathrm{x}(17) . * \mathrm{~s} .{ }^{\wedge} 2+\mathrm{x}$ $(18) . * \mathrm{~s}$; 
$\operatorname{int} x=\sin ($ theta $)$

$82 \quad$ inty $=-\sin ($ phi $) \cdot * \cos ($ theta $)$;

83 intz $=\cos ($ theta $) \cdot * \cos ($ phi $)$;

84

$85 \%$ Computation of end conditions given input beam shape.

$86 \quad \mathrm{xs}=\operatorname{trapz}(\mathrm{s}$, intx $)$;

$87 \quad$ ys $=\operatorname{trapz}(\mathrm{s}$, inty $)$;

$88 \quad \mathrm{zS}=\operatorname{trapz}(\mathrm{s}$, intz $)$;

89

$90 \%$ Apply the constraints to the result.

$91 \quad \operatorname{ceq}(1)=\mathrm{xs}-\mathrm{xL}$;

$92 \operatorname{ceq}(2)=y s-y L ;$

$93 \operatorname{ceq}(3)=\mathrm{zs}-\mathrm{zL}$; 


\section{Appendix $\mathrm{C}$}

\section{Abaqus Scripting Code}

\section{Automation of Abaqus Simulations}

The following code is written in the Python programming language. This code consists of a series of nested FOR loops which iterate through the various model parameters, compute the shape of the bladder segment, mesh the segment, apply the load, and submit the simulation. The results of each run of the simulation are written to a unique. ODB file.

$1 \quad \#-*-$ coding: mbcs $-*-$

2 from part import $*$

3 from material import $*$

4 from section import $*$

5 from assembly import $*$

6 from step import *

7 from interaction import $*$

8 from load import * 
9 from mesh import $*$

10 from optimization import $*$

11 from job import *

12 from sketch import $*$

13 from visualization import $*$

14 from connectorBehavior import *

15

16 import math

17 \#import time

18 \# SET VARIABLES

19 EdgeLengths $=[0.25,0.5,0.75,1.0,1.25,1.5]$

20 BraidAngles $=[5,15,25,35,45,55]$

${ }_{21}$ Thicknesses $=[0.05,0.1,0.15,0.2,0.25]$

${ }_{22}$ Pressures $=[0.2,0.4,0.6,0.8,1.0] \quad$ \# Units are $\mathrm{MPa}$

$23 \mathrm{E}=6.5$

${ }_{24} \mathrm{nu}=0.5$

25

$26 \quad \mathrm{i}=1$

27 for EdgeLength in EdgeLengths:

$$
\text { for BraidAngle in BraidAngles: }
$$

for Thickness in Thicknesses:

for Pressure in Pressures:

$\mathrm{x}=$ EdgeLength $*$ math.cos(BraidAngle $*$ math.pi/180)

$\mathrm{y}=$ EdgeLength $*$ math.sin(BraidAngle $*$ math.pi/180)

xmidpoint $=$ EdgeLength $/ 2 *$ math.cos $($ BraidAngle $*$ math . $\mathrm{pi} / 180)$

ymidpoint $=$ EdgeLength $/ 2 *$ math.sin $($ BraidAngle $*$ math . $\mathrm{pi} / 180)$ 
\# CREATE THE MODEL AND DEFINE EDGE LENGTH, BRAID ANGLE, AND THICKNESS mdb.models['Model -1 '].ConstrainedSketch(name=' _-profile_-', sheetSize $=5.0$ ) mdb.models['Model-1'].sketches[' -_profile_-' ]. Line( point $1=(-\mathrm{x}, 0)$, point $2=($ $0, \mathrm{y})$ )

mdb.models['Model-1'].sketches[' -_profile_-- ']. Line( point $1=(0, y), \operatorname{point} 2=($ $\mathrm{x}, 0)$ )

mdb.models['Model-1'].sketches[' -_profile_-- ']. Line( point $1=(\mathrm{x}, 0)$, point $2=($ $0,-\mathrm{y}))$

mdb.models['Model-1'].sketches[' -_profile_-' ]. Line( point $1=(0,-y)$, point $2=($ $-\mathrm{x}, 0)$ )

mdb.models['Model -1 '] $]$ Part(dimensionality $=$ THREE_D , name $=$ 'Part -1 ', type $=$ DEFORMABLE_BODY) mdb.models['Model-1'].parts['Part-1']. BaseSolidExtrude $($ depth$=$ Thickness, sketch $=$ mdb.models['Model-1'].sketches[' --profile_-']) del mdb.models['Model-1'].sketches[' _-profile_-']

\# SPECIFY THE ELASTIC MATERIAL PROPERTIES

mdb.models['Model-1'].Material(name='Material-1') mdb.models['Model-1'].materials['Material-1'].Elastic( table $=((\mathrm{E}, \mathrm{nu}))$, 
\# SECTION AND ASSIGN TO PART

mdb.models['Model-1'].HomogeneousSolidSection( material $=$ 'Material -1 ', name $=$

'Section -1 ', thickness $=$ None)

mdb.models['Model-1'].parts['Part-1'].

SectionAssignment $($ offset $=0.0$, offsetField $="$, offsetType=MIDDLE_SURFACE, region $=$ Region ( cells $=$ mdb.models['Model -1 '].parts['Part-1'].cells . findAt $(((0.0$, $0.0,0.0),), \quad)$ ), sectionName $=$ 'Section -1 ', thicknessAssignment= FROM_SECTION)

\# CREATE INSTANCE AND STATIC STEP mdb.models['Model-1'].rootAssembly.

DatumCsysByDefault(CARTESIAN) mdb.models['Model-1'].rootAssembly.Instance( dependent $=\mathrm{OFF}$, name $=$ 'Part $-1-1$ ', part $=$ mdb.models['Model -1 '].parts['Part -1 '] $)$ mdb.models['Model -1 '].StaticStep(name='Step -1 ', previous $=$ 'Initial')

\# SET THE PRESSURE LOAD AND BOUNDARY CONDITIONS

mdb.models['Model-1'].Pressure(amplitude=UNSET, createStepName $=$ 'Step -1 ', distributionType $=$ UNIFORM, field $="$, magnitude $=$ Pressure, name $=$ 'Load -1 ', region $=$ Region( 
side1Faces $=$ mdb.models['Model -1 '] .rootAssembly. instances['Part-1-1'].faces.findAt( $((0.0, \quad 0.0,0.0)), \quad))$, mdb.models['Model -1 '].PinnedBC $($ createStepName=' Step -1 ', localCsys $=$ None, name $=$ 'BC -1 ', region $=$ Region ( faces $=$ mdb.models ['Model -1 '] $\cdot \operatorname{root}$ Assembly. instances['Part-1-1'].faces.findAt( ((-xmidpoint, ymidpoint, Thickness/2), ), (( xmidpoint, ymidpoint, Thickness/2), ), (( xmidpoint, -ymidpoint, Thickness/2), ), ((xmidpoint, -ymidpoint, Thickness/2), ), )$)$

\# SEED THE INSTANCE AND MESH IT (MIGHT CHANGE MESH SIZE HERE)

mdb.models['Model-1'].rootAssembly.seedPartInstance( deviationFactor $=0.1$, regions $=($ mdb.models['Model -1 ']. rootAssembly. instances ['Part $-1-1$ '], ), size $=$ $0.05)$ mdb.models['Model-1'].rootAssembly.generateMesh( regions $=($ mdb.models['Model-1'].rootAssembly.instances['

$$
\text { Part-1-1'], )) }
$$

\# CREATE THE JOB AND SUBMIT

jobname $=$ 'Job $-\% \mathrm{~s}$ ' \% i

mdb.Job(atTime $=$ None, contactPrint $=\mathrm{OFF}$, description $=$ ", echoPrint $=\mathrm{OFF}$, 
94

96

97

98

99

100

101

102 \# END OF FOR LOOPS $\mathrm{i}=\mathrm{i}+1$

explicitPrecision $=$ SINGLE, getMemoryFromAnalysis=True, historyPrint $=$ $\mathrm{OFF}$, memory $=90$, memoryUnits $=$ PERCENTAGE, model $=$ 'Model -1 ', modelPrint $=\mathrm{OFF}$, multiprocessingMode $=$ DEFAULT, name=jobname, nodalOutputPrecision=SINGLE, numCpus $=1$, queue $=$ None, scratch $="$, type $=$ ANALYSIS, userSubroutine=", waitHours $=0$, waitMinutes $=0$ ) mdb.jobs[jobname].submit (consistencyChecking $=\mathrm{OFF})$ \#time.sleep(30)

\section{Post Processor Script}

After all of the simulations are complete, the following post processing script written in the Python programming language is used. The script opens each of the .ODB files generated previously, extracts the von Mises stress data, and writes it to a text file for later use.

1 \# READ OUTPUT FROM ODB RESULTS FILES

2 import odbAccess

3

4 maxStress $=[]$ \# Initialize the variable of interest.

${ }_{5}$ for $\mathrm{j}$ in range(1): \# Just need to edit this with actual number of Job output files . 
$6 \quad$ jindex $=\mathrm{j}+1$ \# Adjust index to match job name convention used in parametric study.

$7 \quad$ jobname='Job-\%s.odb' \%jindex

$8 \quad$ stressField $=$ session.openOdb(name $=$ jobname $).$ steps['Step -1 ']. frames[-1].fieldOutputs['S'] \# Define path to stored data.

$9 \quad$ stressVector $=[]$ \# Initialize a temporary variable.

10 for $\mathrm{i}$ in range(len(stressField . values)): \# Loop through all the elements in the model.

$11 \quad$ vonMises=stressField.values[i]. mises \# Extract variable of interest (von Mises stress in this case). stressVector . insert (i, vonMises) \# Put that variable in a the temporary list.

$13 \operatorname{maxS}=\max ($ stressVector) \# Looking for max value in this case. 14 maxStress.insert(jindex, maxS) \# Store that max value in a list. 15 \# end for loop

17 output=file('/some/path/Results.txt','w') \# Once all of the job files are processed, write max stress list to text file.

for item in maxStress:

print $>>$ output, item

\# end output for loop

output.close () \# Close the text file. 